University of Tennessee Health Science Center

UTHSC Digital Commons

$12-2013$

\title{
Identification of Novel HIF1A Target Genes That Regulate Tumor Progression and Metastasis
}

\author{
Danielle L. Peacock \\ University of Tennessee Health Science Center
}

Follow this and additional works at: https://dc.uthsc.edu/dissertations

Part of the Genetic Processes Commons, and the Neoplasms Commons

\section{Recommended Citation}

Peacock, Danielle L. , "Identification of Novel HIF1A Target Genes That Regulate Tumor Progression and Metastasis" (2013). Theses and Dissertations (ETD). Paper 358. http://dx.doi.org/10.21007/ etd.cghs.2013.0241.

This Dissertation is brought to you for free and open access by the College of Graduate Health Sciences at UTHSC Digital Commons. It has been accepted for inclusion in Theses and Dissertations (ETD) by an authorized administrator of UTHSC Digital Commons. For more information, please contact jwelch30@uthsc.edu. 


\title{
Identification of Novel HIF1A Target Genes That Regulate Tumor Progression and Metastasis
}

\begin{abstract}
Hypoxia is a hallmark of most solid tumors. In response to hypoxic stress tumor cells adapt by regulating survival, metabolism and angiogenesis. The heterodimeric Hypoxialnducible Factor (HIF) transcription factors are the master regulators of this response. HIFs play key roles in many critical aspects of cancer biology including angiogenesis, stem cell maintenance, metabolic reprogramming, invasion, metastasis and resistance to radiation therapy and chemotherapy. Overexpression of HIF- $1 \alpha$ and HIF-2 $\alpha$ has been documented in multiple human cancers and HIF-1 protein is over-expressed in $\sim 30 \%$ of primary breast tumors and $\sim 70 \%$ of metastases, which independently correlates with poor prognosis and decreased survival in patients. A precise role for HIF-2 in breast cancer is still being elucidated.
\end{abstract}

Our lab has established primary mammary tumor epithelial cells (MTECs) from late stage carcinomas originating in PyMT+; Hif1a floxed mice. These MTECs were exposed to either Adenovirus-beta-gal or -Cre to create wild-type (WT) and knockout (KO) cells, respectively. Deletion of HIF-1 activity reduced primary tumor growth by $\sim 60 \%$ and the formation of lung macrometastases originating from mammary fat pad tumors by $>90 \%$. In addition, deletion of Hif1a reduced mammary tumorsphere formation efficiency (TSE) in vitro and tumor initiating cell (TIC) frequency in vivo. In contrast, in triple negative models of breast cancer, knockdown of HIF1A had the opposite phenotype, increasing TSE in vitro and increased primary mammary tumor growth in vivo. ITGA6 (CD49f) was identified as one HIF-1 $\alpha$-dependent cancer stem cell marker that enriches for both primary mammary tumor growth and metastasis to the lung. Microarray profiling conducted to identify genes differentially expressed between PyMT WT and KO cells and endstage WT and KO tumors revealed several genes that were down-regulated in both data sets in response to deletion of Hif1a. One mRNA of particular interest, creatine kinase brain isoform (Ckb) was downregulated in KO cells $>100$ fold and $>2$ fold in end-stage tumors. Transplantation of Ckb knockdown (KD) PyMT cells to the mammary fat pad delayed initiation of palpable tumors by $>30$ days. When cells were introduced into the circulation via tail vein injection, $20 \%$ of mice injected with $\mathrm{Ckb} \mathrm{KD}$ cells developed lung metastases whereas $100 \%$ of mice injected with WT cells developed metastases. Likewise, when mice injected with WT PyMT cells were treated daily with a CKB chemical inhibitor, cyclocreatine (cCr), lungs of vehicle treated (saline) mice were almost completely covered with surface metastases, while lungs of cCr treated mice contained very few metastases.

Overall, our data show that HIF-1 $\alpha$ strongly promotes mammary tumor initiation, progression and metastasis, in part through regulation of TIC activity. Metastasis is the major cause of mortality in breast cancer patients. Further characterization of the distinct roles of HIF- $1 \alpha$ versus HIF- $2 \alpha$ in breast cancer and metastasis, and genes downstream of the HIFs, such as ITGA6 (CD49f) and CKB, that play a key role in driving tumor initiation and invasion, are likely to identify new pathways amenable to therapeutic intervention for patients with metastatic breast cancer.

\section{Document Type}

Dissertation

Degree Name

Doctor of Philosophy (PhD)

\section{Program}

Biomedical Sciences 


\section{Research Advisor}

Tiffany N. Seagroves, Ph.D.

\section{Keywords}

Breast Cancer, Metastasis, HIF1A, Hypoxia

\section{Subject Categories}

Diseases | Genetic Processes | Medical Sciences | Medicine and Health Sciences | Neoplasms

\section{Comments}

Two year embargo expired December 2015 
Identification of Novel HIF1A Target Genes That Regulate Tumor Progression and Metastasis

\author{
A Dissertation \\ Presented for \\ The Graduate Studies Council \\ The University of Tennessee \\ Health Science Center \\ In Partial Fulfillment \\ Of the Requirements for the Degree \\ Doctor of Philosophy \\ From The University of Tennessee
}

By

Danielle L. Peacock

December 2013 
Chapter 2 (C) 2010 by Elsevier.

Chapter 3 (C) 2012 by BioMed Central Ltd.

Appendix (C) 2013 by American Association of Cancer Research.

All other material C 2013 by Danielle L Peacock.

All rights reserved. 


\section{DEDICATION}

This is dedicated to my family, my parents Thomas and Kelly for their endless encouragement and support, and Michael Brooks for his love and devotion. 


\section{ACKNOWLEDGEMENTS}

I would like to thank my graduate advisor, Tiffany Seagroves, Ph.D., and my advisory committee: Suzanne Baker, Ph.D.; Bryan Welm Ph.D.; Lorraine Albritton, Ph.D.; Len Lothstein Ph.D.; Tony Marion, Ph.D. and Meiyun Fan, Ph.D. for their instrumental comments, ideas, support and motivation. I would also like to acknowledge Luciana Schwab, $\mathrm{PhD}$ for providing me with daily laboratory help (whether meaningful or menial), encouragement and company. Additionally, I would like to recognize Laura Jensen, Keisha Smith, and Richard Cushing for their helpful assistance on all projects presented here. Finally, I would like thank all of the faculty and staff of the University of Tennessee Health Science Center, Center for Cancer Research for providing a friendly environment for learning and research; and in particular, Junming Yue, Ph.D. for help with DNA cloning and lentiviral preparation, and everyone at the Molecular Resource Center for their support with RT-PCR, microarray analysis and DNA sequencing. 


\begin{abstract}
Hypoxia is a hallmark of most solid tumors. In response to hypoxic stress tumor cells adapt by regulating survival, metabolism and angiogenesis. The heterodimeric Hypoxia-Inducible Factor (HIF) transcription factors are the master regulators of this response. HIFs play key roles in many critical aspects of cancer biology including angiogenesis, stem cell maintenance, metabolic reprogramming, invasion, metastasis and resistance to radiation therapy and chemotherapy. Overexpression of HIF- $1 \alpha$ and HIF-2 $\alpha$ has been documented in multiple human cancers and HIF-1 $\alpha$ protein is over-expressed in $\sim 30 \%$ of primary breast tumors and $\sim 70 \%$ of metastases, which independently correlates with poor prognosis and decreased survival in patients. A precise role for HIF-2 $\alpha$ in breast cancer is still being elucidated.
\end{abstract}

Our lab has established primary mammary tumor epithelial cells (MTECs) from late stage carcinomas originating in PyMT+; Hifl a floxed mice. These MTECs were exposed to either Adenovirus-beta-gal or -Cre to create wild-type (WT) and knockout (KO) cells, respectively. Deletion of HIF-1 activity reduced primary tumor growth by $\sim 60 \%$ and the formation of lung macrometastases originating from mammary fat pad tumors by $>90 \%$. In addition, deletion of Hifla reduced mammary tumorsphere formation efficiency (TSE) in vitro and tumor initiating cell (TIC) frequency in vivo. In contrast, in triple negative models of breast cancer, knockdown of $H I F 1 A$ had the opposite phenotype, increasing TSE in vitro and increased primary mammary tumor growth in vivo. ITGA6 (CD49f) was identified as one HIF-1 $\alpha$-dependent cancer stem cell marker that enriches for both primary mammary tumor growth and metastasis to the lung. Microarray profiling conducted to identify genes differentially expressed between PyMT WT and KO cells and end-stage WT and KO tumors revealed several genes that were down-regulated in both data sets in response to deletion of Hifla. One mRNA of particular interest, creatine kinase brain isoform $(\mathrm{Ckb})$ was down-regulated in $\mathrm{KO}$ cells $>100$ fold and $>2$ fold in end-stage tumors. Transplantation of $C k b$ knockdown (KD) PyMT cells to the mammary fat pad delayed initiation of palpable tumors by $>30$ days. When cells were introduced into the circulation via tail vein injection, $20 \%$ of mice injected with $C k b \mathrm{KD}$ cells developed lung metastases whereas $100 \%$ of mice injected with WT cells developed metastases. Likewise, when mice injected with WT PyMT cells were treated daily with a $\mathrm{CKB}$ chemical inhibitor, cyclocreatine $(\mathrm{cCr})$, lungs of vehicle treated (saline) mice were almost completely covered with surface metastases, while lungs of cCr treated mice contained very few metastases.

Overall, our data show that HIF-1 $\alpha$ strongly promotes mammary tumor initiation, progression and metastasis, in part through regulation of TIC activity. Metastasis is the major cause of mortality in breast cancer patients. Further characterization of the distinct roles of HIF- $1 \alpha$ versus HIF-2 $\alpha$ in breast cancer and metastasis, and genes downstream of the HIFs, such as ITGA6 (CD49f) and CKB, that play a key role in driving tumor initiation and invasion, are likely to identify new pathways amenable to therapeutic intervention for patients with metastatic breast cancer. 


\section{TABLE OF CONTENTS}

CHAPTER 1. INTRODUCTION ................................................................................

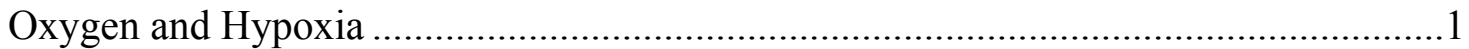

Oxygen and Hypoxia: Effects on Cancer Therapy ...................................................... 1

Regulation of Hypoxia Inducible Factors (HIFs) .......................................................2

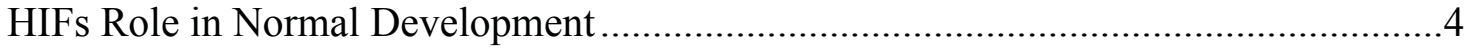

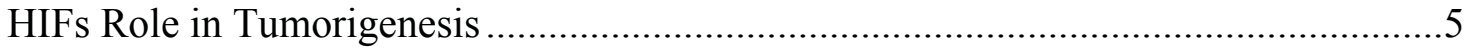

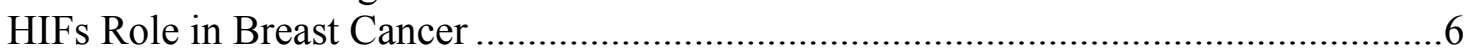

Significance of the Hypoxic Response in Metastasis .................................................8

Identification of Novel or Understudied HIF-Regulated Genes to Identify New

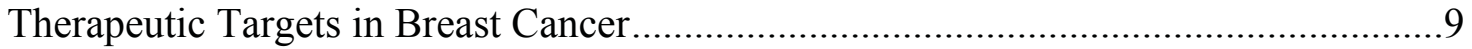

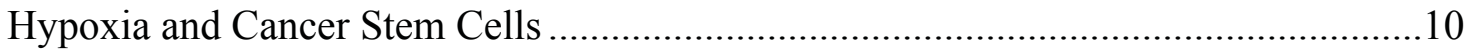

Cancer Stem Cell Theory and Regulation of Breast Cancer Stem Cell Activity by

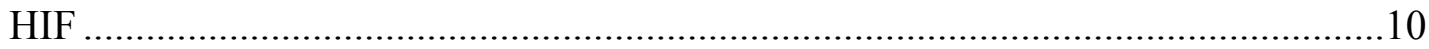

HIF Regulation of Cancer Stem Cells in the MMTV-PyMT Mouse Model .............11

Moving into Translational Research: Investigating the Role of HIF-1 and HIF-2 in Human Models of Luminal and Triple Negative Breast Cancers...............................11

Hypothesis and Specific Aims ........................................................................... 13

\section{CHAPTER 2. VHL DELETION IMPAIRS MAMMARY ALVEOLOGENESIS} BUT IS NOT SUFFICIENT FOR MAMMARY TUMORIGENESIS .....................14

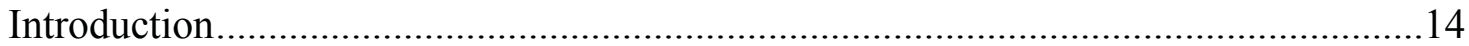

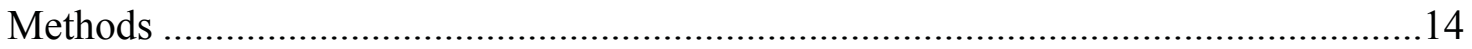

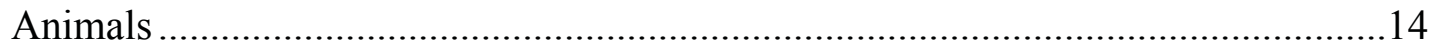

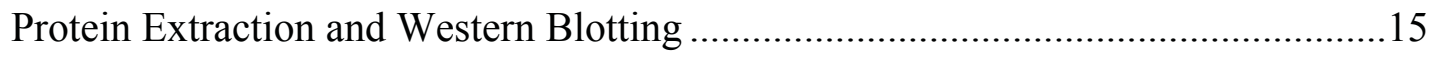

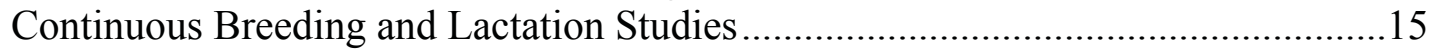

Tissue-Harvest, Histology and Whole-Slide Scanning .........................................15

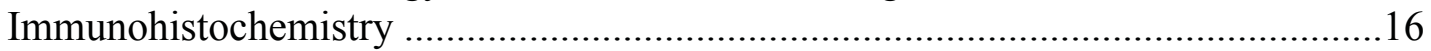

Automated Analysis of Epithelial Cell Content........................................................16

Gene Expression Analysis by RT-PCR …........................................................... 17

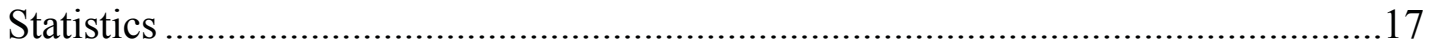

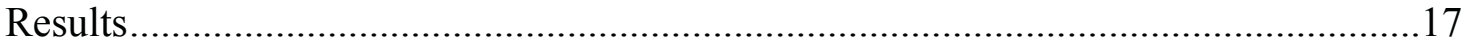

VHL Expression Increases during Pregnancy and Decreases during Involution ......17

Conditional Deletion of Vhlh Impairs MEC Proliferation and Differentiation

during Pregnancy .................................................................................... 18

Deletion of Vhlh Results in Subtle Changes in Secretory Activity during First

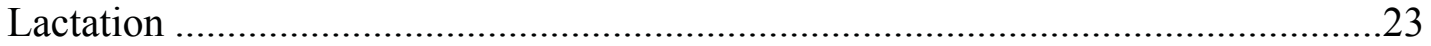

Deletion of Vhlh Contributes to Progressive Lactation Failure ...............................23

Deletion of Vhlh Does Not Promote Mammary Carcinoma in Aged Females..........27

Constitutive Expression of HIF-1 $\alpha$, GLUT1 and VEGF, Increased Microvessel

Density and Enhanced Recruitment of Leukocytes in Response to Vhlh Deletion ...29

Epithelial-Specific Deletion of Vhlh Induces a Stromal Response..........................29

Conditional Co-Deletion of Hifla Does Not Rescue the VHL-Dependent

Phenotypes 


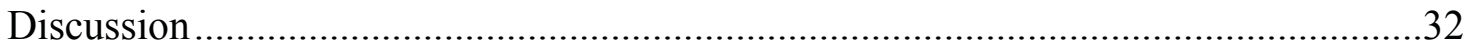

A Role for VHL in Mammary Progenitor Cells .......................................................34

A Key Role for Local VEGF in Regulating the Mammary-Associated

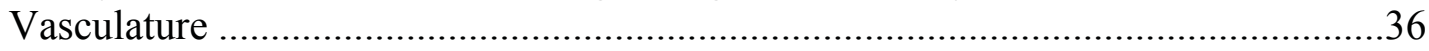

Hifla Deletion Cannot Rescue the Vhlh Phenotype, a Role for Other VHL-

Regulated Targets .............................................................................................36

A Role for VHL in the Prolactin Response .........................................................37

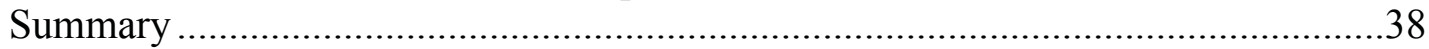

\section{CHAPTER 3. HYPOXIA-INDUCIBLE FACTOR $1 \alpha$ PROMOTES PRIMARY TUMOR GROWTH AND TUMOR-INITIATING CELL ACTIVITY IN BREAST CANCER ...........................................................................................................39}

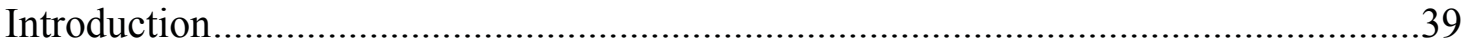

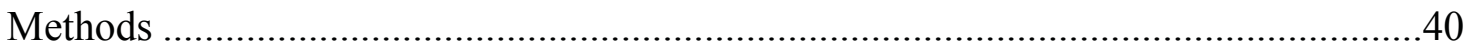

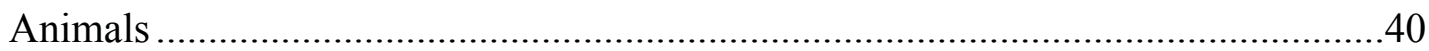

Establishing Hifla Wild-Type and Knockout Mammary Tumor Epithelial Cells ....40

Western Blot Analysis .........................................................................................4

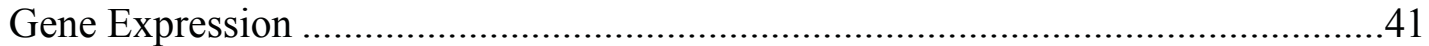

Cell Growth and Invasion Assays ...........................................................................42

Mammary Tumor Epithelial Cell Transplant into FVB Recipients ............................42

Tissue Histology and Immunostaining ................................................................43

Lung Metastasis and Survival Studies ...................................................................43

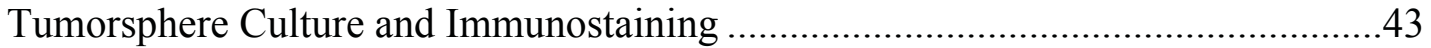

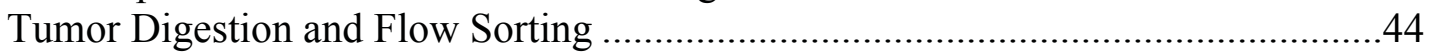

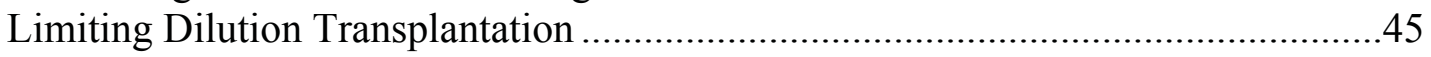

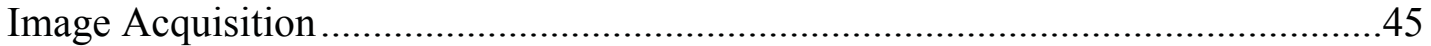

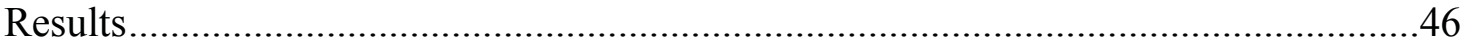

A Mammary Tumor Model with Constitutively Deleted HIF-1 $\alpha$..............................46

HIF-1 $\alpha$ Promotes Cell Growth and Invasion.............................................................51

Expression of Basal Markers Is Reduced in Cultured Knockout Cells ......................51

Primary Mammary Tumor Growth Is HIF-1 $\alpha$-Dependent.........................................53

HIF-1 $\alpha$ Promotes an Epithelial-to-Mesenchymal Transition Phenotype and

Regulates K5 Expression in vivo........................................................................59

Constitutive Hifla Deletion Represses Metastasis and Prolongs Survival ................59

Deletion of Hifla Decreases Tumorsphere Formation ................................................63

HIF-1 $\alpha$-Dependent Regulation of CD133 and the Notch Pathway .............................69

HIF-1 $\alpha$ Promotes Tumor-Initiating Cell Frequency in vivo ......................................72

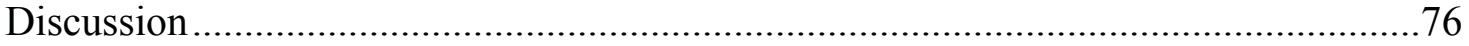

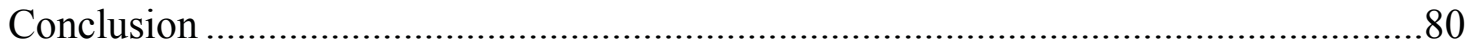

\section{CHAPTER 4. ITGA6 (CD49F) IS DIRECTLY REGULATED BY HYPOXIA- INDUCIBLE FACTORS (HIFS) AND PROMOTES TUMOR INITIATION AND LUNG METASTASIS ..........................................................................................82}

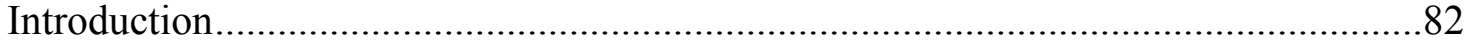

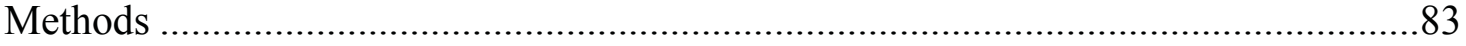

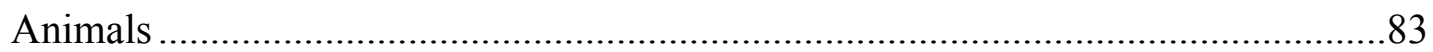




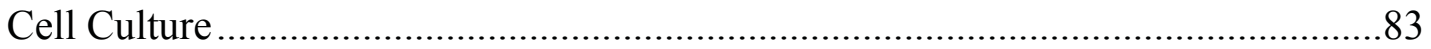

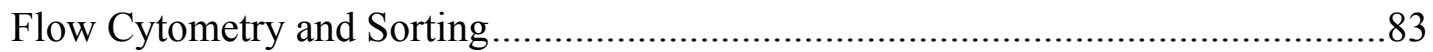

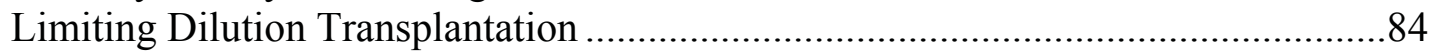

Tail Vein Injection of MDA-MB-231 Cells into NSG Recipients ..............................84

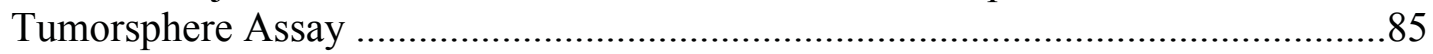

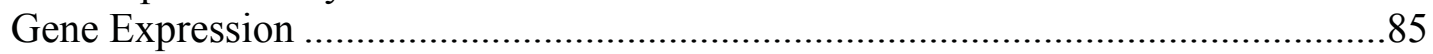

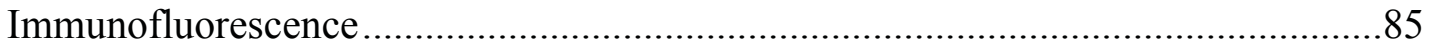

Promoter Analysis and Chromatin Immunoprecipitation (ChIP) ...............................85

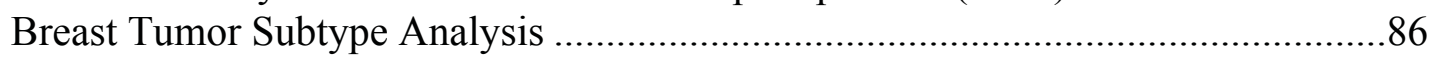

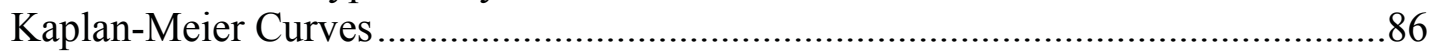

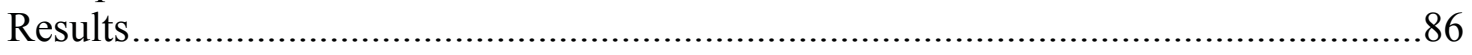

Deletion of HIF 1A Down-Regulates CD49f Expression .........................................86

Deletion of HIF1A Alone or Together with HIF2A, Results in Decreased

Expression of CD49f by the Human Breast Cancer Cell Line MDA-MB-231 .........89

ITGA6 (CD49f) Is a Direct HIF Transcriptional Target Gene...................................91

CD49f Promotes Primary Tumor Formation and Increases TIC Frequency in the

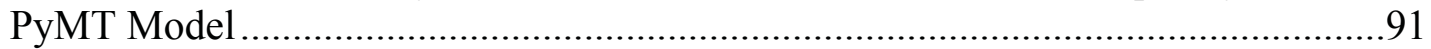

CD49f Promotes Lungs Metastases Induced by Tail Vein Injection of MDA-

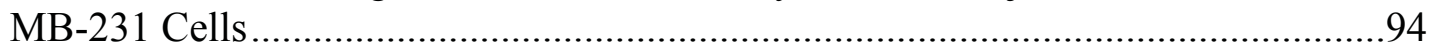

CD49f Expression Is Enriched in Specific Subtypes of Human Breast Cancers and Correlates with Decreased Survival ............................................................96

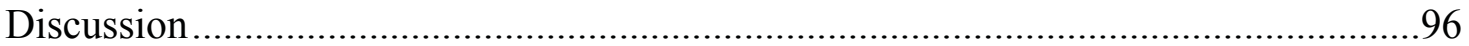

\section{CHAPTER 5. IDENTIFYING NOVEL OR UNDERSTUDIED HIF-} REGULATED GENES.....................................................................................100

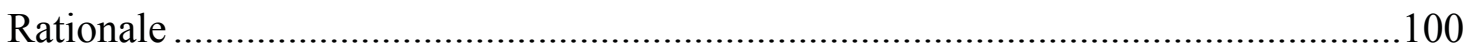

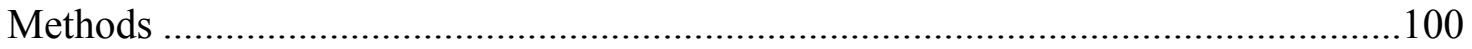

Gene Expression Microarray Analysis ………………….................................100

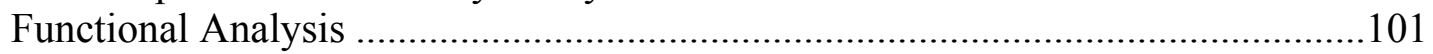

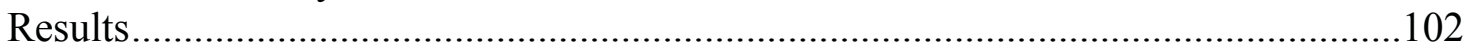

Effects of EGF on HIF-1 $\alpha$-Dependent Gene Expression ......................................102

HIF-1 $\alpha$-Dependent Gene Expression in PyMT MTECs and Tumors ......................105

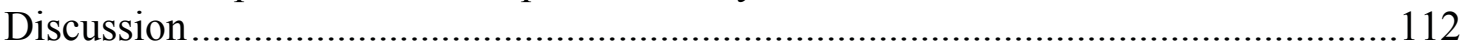

CHAPTER 6. CREATINE KINASE B (CKB) ACTIVITY IS HIF-1DEPENDENT AND IS ESSENTIAL FOR PRIMARY TUMOR GROWTH, CELL INVASION AND METASTASIS TO THE LUNG ...........................................113

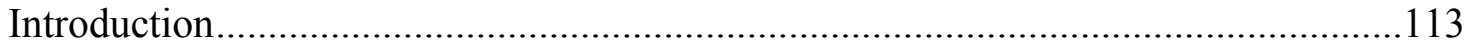

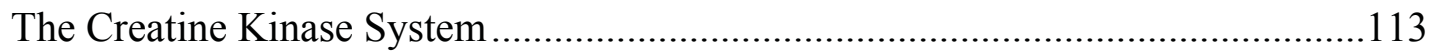

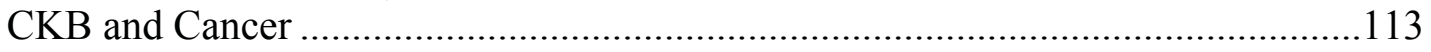

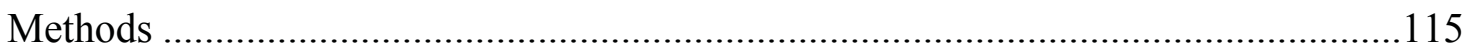

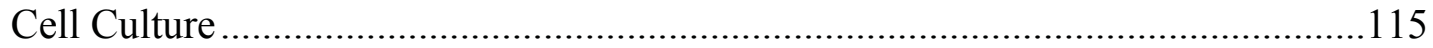

Generation of Ckb Cell Line Variants ................................................................117

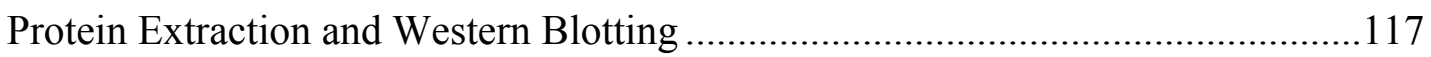

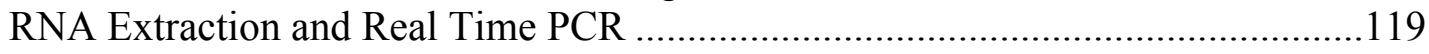

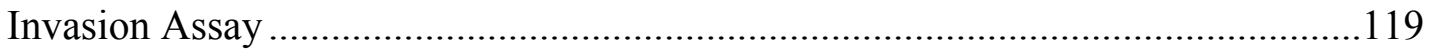


Cell Proliferation Assay ………………….......................................................119

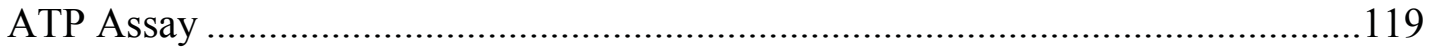

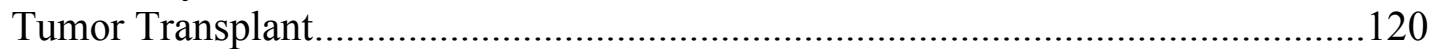

Tail Vein and Cyclocreatine Experiments ...........................................................120

Tissue Harvest, Histology and Immunostaining ........................................................120

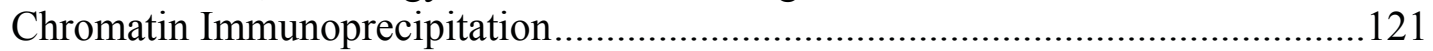

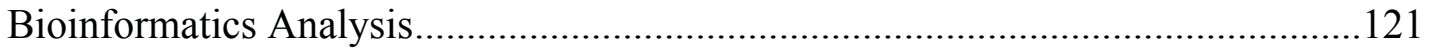

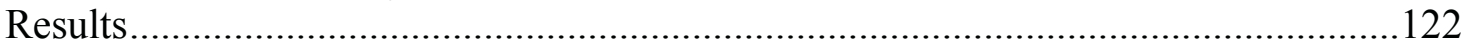

Ckb Expression Is Decreased in Hifla KO Cells and Tumors ...............................122

Knockdown of $C k b$ Reduces Tumor Cell Invasion in vitro......................................122

Loss of $C k b$ Significantly Delays Tumor Formation and Inhibits Lung

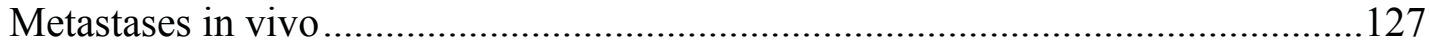

Inhibition of $\mathrm{Ckb}$ with Cyclocreatine Blocks Lung Metastasis ................................127

$C K B$ Expression Correlates with HIFlA mRNA Expression and with Decreased

Relapse Free Survival in Patients with Breast Cancer..............................................127

Is $C k b$ a Direct HIF-1 $\alpha$ Target? ....................................................................133

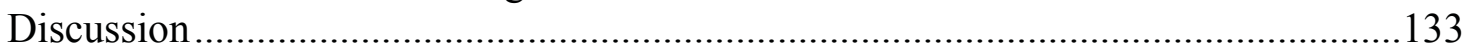

\section{CHAPTER 7. USING HUMAN MODELS TO ELUCIDATE HIF-1 VERSUS}

HIF-2 CONTRIBUTION IN BREAST CANCER...................................................139

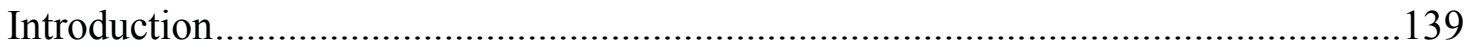

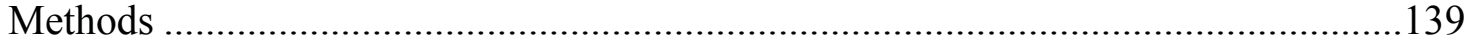

Human Xenograft Maintenance .......................................................................139

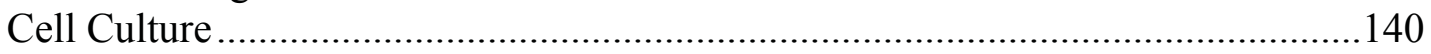

Tumor Cell Transplants into NSG Recipients .....................................................140

Genetic Modification of HIFs in HCI Tumors …………...................................... 140

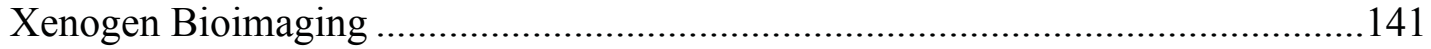

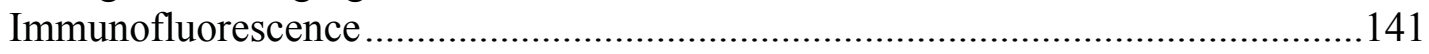

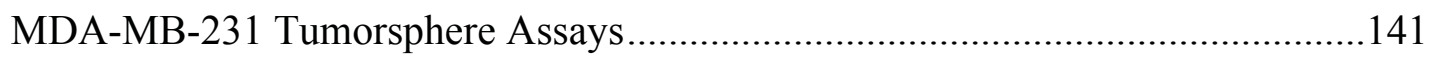

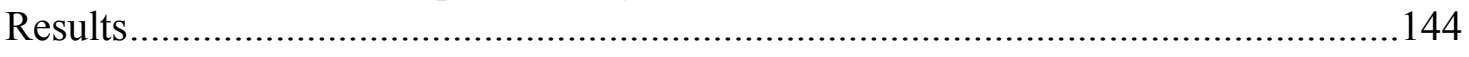

HIF $\alpha$ Expression in Triple Negative Breast Cancer Models .....................................144

Transduction of HCI Tumor Cells with Lentivirus for Live Bioimaging and

Evaluation of Metastatic Spread over Time...........................................................144

HIF1A Knockdown in MDA-MB-231 Cells Increases Primary Tumor Growth ....151

Characterization of Tumor Growth and Metastasis Using the TNBC HCI-10

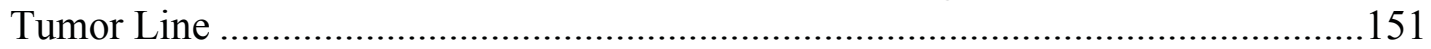

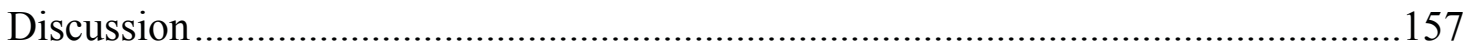

CHAPTER 8. DISCUSSION ........................................................................................158

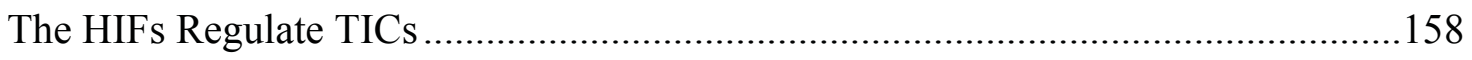

The HIFs Promote Tumor Metastasis .........................................................................159

A Novel HIF-Dependent Gene Involved in Breast Cancer Progression and

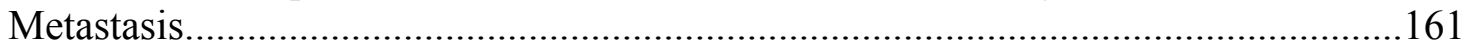

The Differing Roles of the HIFs in Breast Cancer ...................................................162

A Role for the HIFs in Stromal Cells in Supporting Breast Cancer Progression and

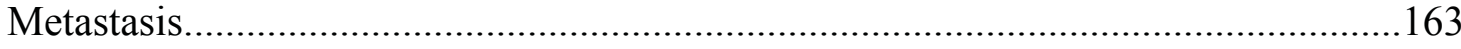


Summary of Major Findings.

CHAPTER 9. REMAINING QUESTIONS AND FUTURE EXPERIMENTS .......166

Is CD49f Alone a Driver of Tumor Progression and Metastasis, or Does it Require the Presence of CD24?

Can Re-Expression of Ckb in PyMT HIF-1 $\alpha$ KO or shc61 KD Cells Rescue the

Tumor and Metastasis Phenotypes?.

Is the Ckb shRNA Targeting Sequence (c61) Specific to Ckb, or Are There Off-

Target Effects?

Can Treating Primary Tumors with Cyclocreatine Inhibit Primary Tumor Growth

as well as Lung Metastases?.

Is CKB a Direct HIF Target Gene?

Are There Additional Understudied Genes Revealed by Array Profiling That Could Provide Additional Targets for Successful Breast Cancer Therapy?

Are There Additional Cells Lines to Use as Models That Would Replicate My

Results Derived from MDA-MB-231 Cells?.

Which Additional Studies Can Be Performed to Better Define the Potentially

Opposing Roles of the HIF $\alpha$ Proteins in TNBC?

LIST OF REFERENCES

\section{APPENDIX. BREAST TUMOR KINASE (BRK/PTK6) IS A MEDIATOR OF HYPOXIA/HIF-ASSOCIATED BREAST CANCER PROGRESSION.}

Abstract.

Introduction

Materials and Methods

Generation of Modified MDA-MB-231 Cells and Cell Culture

Cell Proliferation Assay

Protein Extraction

Immunoblotting.

qPCR

ChIP Assays

Transgenic Mice and Generation of Tumors in NOD/scid/gamma Recipients .......200

Immunostaining

Kaplan-Meier Curves

Statistical Analysis

Brk Is Upregulated in Response to Hypoxia and Cellular Stress .........................201

Brk Is a Novel, Direct HIF Transcriptional Target Gene ....................................206

Ectopic Brk Expression Enhances Growth of HIF1A/HIF2A Knockout Tumors in vivo

Brk Increases the Appearance of Basal-like Mammary Tumors in MET ${ }^{\text {mut }}$ Mice ..213

Discussion.... 


\section{LIST OF TABLES}

Table 3-1. Estimation of TIC frequency of HIF-1 $\alpha$ WT and KO MTECs.

Table 4-1. ELDA analysis of TIC frequency in CD24 $4^{\text {high }} / \mathrm{CD} 49 \mathrm{f}^{+}$or $\mathrm{CD} 24^{\text {low }} / \mathrm{CD} 49 \mathrm{f}^{\text {neg }}$ derived tumors at day 51 post transplant.

Table 5-1. Summary of culture conditions used for cells harvested for microarray profiling.

Table 5-2. All pairwise comparisons from datasets from cells...............................102

Table 5-3. Top 10 differentially regulated genes between cells and tumors................108

Table 5-4. DAVID functional analysis of differentially regulated HIF-1 $\alpha$ genes. ......109

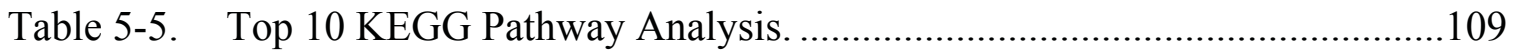

Table 5-6. GSEA Analysis of HIF-1 $\alpha$ regulated genes enriched in WT cells and tumors.

Table 6-1. Summary of surface lung metastases in mice treated with saline versus cyclocreatine for 21 days.

Table 6-2. Summary of surface lung metastases in mice treated with saline versus cyclocreatine for 21 days (day 1 start) or cyclocreatine for 14 days (day 7 start).

Table A-1. Enhanced metastasis in Brk x MET ${ }^{\mathrm{Mut}}$ mice relative to $\mathrm{MET}^{\mathrm{Mut}}$ and WAP-Brk mice. 


\section{LIST OF FIGURES}

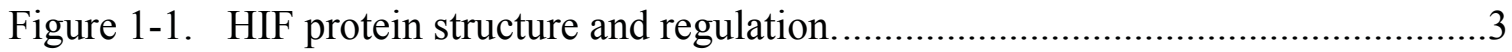

Figure 1-2. Overview of differences between triple negative and luminal breast cancer.

Figure 2-1. Expression of murine VHL increases over the course of normal mammary gland development as epithelial cell content increases.

Figure 2-2. Reduced alveolar proliferation and differentiation in response to Vhlh deletion via MMTV-Cre.

Figure 2-3. Deletion of Vhlh via Wap-Cre phenocopies results obtained with MMTV-Cre

Figure 2-4. Mosaic nature of Cre expression under control of the Wap promoter. .26

Figure 2-5. In multiparous dams, Vhlh $^{-/ \mathrm{MEC}}$ glands exhibit evidence of mild dysplasia. .28

Figure 2-6. Changes in HIF-1 $\alpha$, GLUT1 and VEGF expression, microvessel density and immune cell infiltration in multiparous mice.

Figure 2-7. Co-deletion of Vhlh and Hifla via does not rescue the $V h l$ lactation phenotype.

Figure 3-1. HIF-1 $\alpha$ expression increases during tumor progression in the MMTVPyMT model and confirmation of HIF-1 $\alpha$ deletion.

Figure 3-2. Effect of HIF-1 $\alpha$ deletion upon growth and invasion.

Figure 3-3. HIF-1 $\alpha$ protein expression increases in response to EGF treatment at normoxia, and EGF prolongs HIF-1 $\alpha$ stabilization at hypoxia. .50

Figure 3-4. Loss of basal marker expression in cultured HIF-1 KO cells. 52

Figure 3-5. Deletion of Hifla decreases primary tumor growth. .54

Figure 3-6. Histology of HIF-1 $\alpha$ WT and KO end-stage tumors .56

Figure 3-7. Ki67 and activated caspase-3 immunostaining in end-stage PyMT tumors

Figure 3-8. Expression of $\mathrm{p} 63$ and ER $\alpha$ in WT and KO end-stage PyMT tumors. .58 
Figure 3-9. Loss of cells expressing K14 or K5 in HIF-1 KO tumors is accompanied by a reduction in the expression of markers of EMT.

Figure 3-10. HIF-1 $\alpha$ expression in tumor epithelium is required for metastasis.

Figure 3-11. HIF-1 $\alpha$ promotes tumorsphere formation in cultured cells and in freshly isolated tumor cells independent of oxygen tension.

Figure 3-12. HIF-1 $\alpha$ expression in tumorspheres cultured acutely or chronically at hypoxia.

Figure $3-13$. Clonal expansion of tumorspheres........................................................68

Figure 3-14. CD133 expression in PyMT MTECs is regulated by HIF-1 .......................70

Figure $3-15$. HIF-1 $\alpha$ regulates tumor-initiation potential in vivo. .............................. 74

Figure 4-1. ITGA6 (CD49f) is down-regulated upon deletion of Hifla in PyMT cells, tumors and in HIF-1 $\alpha$ KO tumorspheres.

Figure 4-2. CD49f expression is reduced in HIF $1 A$ and HIF $2 A$ double knockdown MDA-MB-231 cells and tumors.

Figure 4-3. ITGA6 (CD49f) is a direct HIF target gene in MDA-MB-231 cells.

Figure 4-4. PyMT CD24hi CD49f ${ }^{\dagger}$ cells are enriched for TIC activity in vitro and in vivo.

Figure 4-5. FACS-sorted CD49f ${ }^{\text {high }}$ cells exhibit increased lung metastatic potential in a tail vein assay.

Figure 4-6. Correlation between ITGA6 and HIFIA expression and survival in breast cancer patients.

Figure 5-1. Comparison of up-regulated genes in HIF-1 $\alpha$ WT cells in the presence of EGF \pm hypoxia.

Figure 5-2. HIF-1 $\alpha$-dependent genes in the presence and absence of EGF and hypoxia.

Figure 5-3. Differentially regulated genes between HIF-1 $\alpha$ WT and KO cells at normoxia and hypoxia.

Figure 5-4. Differentially regulated genes in common between PyMT MTECs and end stage tumors.

Figure 6-1. Overview of creatine kinase enzymatic activity. 
Figure 6-2. Structure of $C k b$ and location of shRNA targeting sequences

Figure 6-3. Vectors used to create $C k b$ cell line variants.

Figure 6-4. $\quad C k b$ expression in PyMT Hifla WT and KO cells and end-stage tumors. .123

Figure 6-5. Evaluation of 3 independent $C k b$ shRNA vectors to efficiently knockdown CKB in cells transfected with pLKO.1 viral vector DNA......124

Figure 6-6. Effect of $C k b$ knockdown upon growth and invasion.

Figure 6-7. $\quad C k b$ knockdown decreases primary tumor growth and end point tumor wet weight and increases survival time from lung metastases.

Figure 6-8. Knockdown of $C k b$ decreases lung metastasis burden.

Figure 6-9. Inhibition of $\mathrm{Ckb}$ by cyclocreatine $(\mathrm{cCr})$ treatment blocks the formation of lung metastases.

Figure 6-10. $C K B$ correlates with $H I F 1 A$ in primary breast cancer samples.

Figure 6-11. Preliminary ChIP analysis of the $C k b$ promoter.

Figure 7-1. Maps of vectors used for luciferase labeling.

Figure 7-2. Schematic of lentivirus transduction of HCI tumors in stem cell conditions.

Figure 7-3. HIF $\alpha$ expression in HCI tumors of specific subtypes.

Figure 7-4. Xenogen bioimaging of HCI-2 Luc2+ tumor cells.

Figure 7-5. Xenogen bioimaging of HCI-2+shHIF-1 $\alpha$ or EV transduced tumors over time.

Figure 7-6. Comparison of tumor growth and HIF-1 $\alpha$ protein levels by western blotting and IHC in third passage HCI-2 tumors.

Figure 7-7. After selecting for the most efficient HIF1A knockdown HCI-2 tumor, shHIF1A repassaged $\mathrm{P} 4$ tumors grow faster than EV repassaged tumors. 150

Figure 7-8. Characterization of HIF $\alpha$ knockdown phenotypes in MDA-MB-231 cells.

Figure 7-9. Phenotypes following knockdown of HIF-1 $\alpha$ and HIF- $2 \alpha$ in MDA-MB231 cells.

Figure 7-10. HCI-10 Luc2+ P1 tumor growth and metastasis over time. 155 
Figure 7-11. Characterization of P2 HCI-10 Luc2+ tumor and AxLN metastasis by

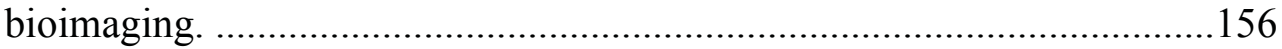

Figure A-1. Brk is upregulated in breast cancers and in human TNBC cell lines..........203

Figure A-2. Hypoxia and other cellular stresses induce Brk expression.........................205

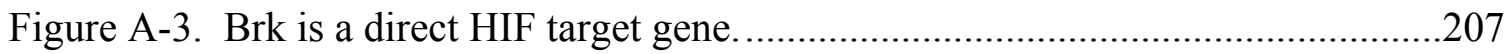

Figure A-4. Sik protein in PyMT+ murine mammary tumors is dependent upon HIF-

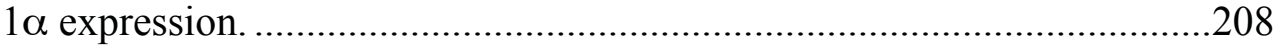

Figure A-5. Brk mRNA and protein expression are ablated upon loss of both HIF-1 $\alpha$

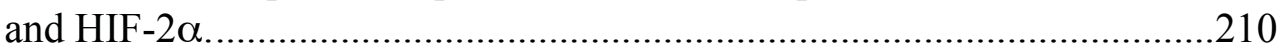

Figure A-6. Brk promotes growth in HIF DKD cells in vitro and in vivo.

Figure A-7. Growth of basal-like mammary tumors that originate in $\mathrm{MET}^{\mathrm{mut}}$ mice is accelerated by expression of the Brk transgene or endogenous Sik. .........214 


\section{LIST OF ABBREVIATIONS}

7-AAD

$\mathrm{ABC}$

ADRP

ALDOC

AML

ANGPTL4

APC

ARNT

AXL

AxLN

BCRP1

bHLH

BNIP3

BNIP3L

BRCA1

BrdU

CA9

$\mathrm{cCr}$

CD24

CD29

CD31

CD44
7-Aminoactinomycin D

Avidin-Biotin Complex

Adipose differentiation related protein

Aldolase C

Acute myeloid leukemia

Angiopoetin like 1

Streptavidin-allophycocyanin

Aryl hydrocarbon nuclear translocator

Tyrosine-protein kinase receptor UFO

Axillary lymph node

Breast carrier resistance protein-1

Basic helix-loop-helix

BCL2/adenovirus E1B 19 kDa protein-interacting protein 3

BCL2/adenovirus E1B $19 \mathrm{kDa}$ protein-interacting protein 3-like

Breast cancer type 1 susceptibility protein

Bromodeoxyuridine

Carbonic anhydrase 9

Cyclocreatine

heat stable antigen

integrin beta 1

Platelet endothelial cell adhesion molecule

Cluster of Differentiation 44 
CD45

CD49f/ITGA6

CD61

CD68

CK

CKB

CKM

CKMT1

CKMT2

CLD

$\mathrm{Cp}$

CRCX4

CRM

CSC

CSN2

CTGF

CTSD

CYPA

DAB

DAPI

DAVID

DCIS

DF
Protein tyrosine phosphatase, receptor type, C

integrin alpha 6

integrin beta 3

Cluster of Differentiation 68

Creatine kinase

Creatine kinase brain isoform

Creatine kinase muscle isoform

Creatine kinase mitochondrial isoform 1

Creatine kinase mitochondrial isoform 2

Cytoplasmic lipid droplets

Crossing point

C-X-C chemokine receptor type 4

Cross reactive material

Cancer stem cell

ß-casein

Connective tissue growth factor

Cathepsin D

Cyclophylin A

3,3'-diaminobenzidine

4',6-diamidino-2-phenylindole

Database for Annotation, Visualization and Integrated Discovery

Ductal carcinoma in situ

Floxed/floxed, Double floxed 


\begin{tabular}{ll} 
DKD & Double knockdown \\
DLL1 & Delta-like protein 1 \\
DN & Double negative \\
DOX & Doxorubicin \\
DP & Double positive \\
EC & Early carcinoma \\
ECL & Enhanced chemiluminescence \\
EGFR & Epidermal growth factor \\
ELDA & Extreme limiting dilution analysis \\
EMB & Embigin \\
EMT & Epithelial to mesenchymal transition \\
EPO & Erythropoietin \\
ER(ESR1) & Estrogen receptor (alpha) \\
ERBB2 & F-erb-b2 avian erythroblastic leukemia viral oncogene homolog 2 \\
ES & Fibronectin \\
EV & Foliend Virus B \\
FACS & Fompty vector \\
FBS & Fluorescence-activated cell sorting \\
FC & Food and Drug cells \\
FDA & Fluorescein isothiocyanate \\
\hline
\end{tabular}


FZD7

GAPDH

GATA3

GFP

GLUT1

GRB7

GSEA

$\mathrm{H} \& \mathrm{E}$

HBSS

HCI

HEPES

HER2

HEY1

HEY2

HIF

HP

HRE

HRP

HSC

IF

IL6

INTS3

JAK2
Frizzled-7

Glyceraldehyde 3-phosphate dehydrogenase

Trans-acting T-cell-specific transcription factor GATA-3

Green fluorescent protein

Glucose transporter 1

Growth factor receptor-bound protein 7

Gene set enrichment analysis

Hematoxylin and eosin

Hank's balanced salt solution

Huntsman Cancer Institute

4-(2-hydroxyethyl)-1-piperazineethanesulfonic acid

Human epidermal growth factor receptor 2

Hairy/enhancer-of-split related with YRPW motif protein 1

Hairy/enhancer-of-split related with YRPW motif protein 2

Hypoxia inducible factor

Hyperplasia

Hypoxia response element

Horseradish peroxidase

Hemapoietic stem cell

Immunofluorescence

Interleukin 6

Integrator complex subunit 3

Janus kinase 2 


\begin{tabular}{|c|c|}
\hline $\mathrm{K} 5 / \mathrm{Krt} 5$ & Cytokeratin 5 \\
\hline $\mathrm{K} 8 / \mathrm{Krt} 8$ & Cytokeratin 8 \\
\hline $\mathrm{K} 14 / \mathrm{Krt} 14$ & Cytokeratin 14 \\
\hline $\mathrm{K} 18 / \mathrm{Krt} 18$ & Cytokeratin 18 \\
\hline KD & Knockdown \\
\hline KEGG & Kyoto Encyclopedia of Genes and Genomes \\
\hline KO & Knockout \\
\hline LC & Late carcinoma \\
\hline LDHA & Lactate dehydrogenase \\
\hline $\mathrm{LN}$ & Lymph node \\
\hline LOX & Lysyl oxidase \\
\hline LRP5 & Low-density lipoprotein receptor-related protein 5 \\
\hline Luc2 & Luciferase \\
\hline MAPK & Mitogen-activated protein kinase \\
\hline MBC & Metastatic breast cancer \\
\hline MEC & Mammary epithelial cells \\
\hline MFI & Mean fluorescence intensity \\
\hline MMP & Matrix metalloproteinase \\
\hline MMTV & Mouse mammary tumor virus \\
\hline MOI & Multiplicity of infection \\
\hline MSC & Mesenchymal stem cell \\
\hline MTD & maximum tolerated dose \\
\hline MTEC & Mammary tumor epithelial cell \\
\hline
\end{tabular}




\begin{tabular}{|c|c|}
\hline NBF & Neutral buffered formalin \\
\hline NOD & Non-obese diabetic \\
\hline NSG & NOD.Cg-Prkdc $c^{\text {scid }} I l 2 r g^{\text {tml } W_{j l} l} / \mathrm{SzJ}$ \\
\hline ODD & Oxygen-dependent degradation domain \\
\hline $\mathrm{OE}$ & Over expression \\
\hline OPN & Osteopontin \\
\hline OS & Overall survival \\
\hline PAS & Per-Arnt-Sim \\
\hline PBS & Phosphate buffered saline \\
\hline $\mathrm{PCr}$ & Phophocreatine \\
\hline PDGFB & Platelet-derived growth factor B \\
\hline PDX & Patient derived xenograft \\
\hline $\mathrm{PE}$ & Phycoerythrin \\
\hline PFA & Paraformaldahyde \\
\hline PGK1 & Phosphoglycerate kinase 1 \\
\hline PHD & Prolyl hydroxylase \\
\hline PHD3/EGLN3 & Prolyl hydroxylase 3 \\
\hline PI & Parity-induced \\
\hline PI3K & Phosphatidylinositol 3-kinase \\
\hline PR & Progesterone receptor \\
\hline PROM1/CD133 & Prominin 1 \\
\hline PTEN & Phosphatase and tensin homolog \\
\hline PVDF & Polyvinylidene difluoride \\
\hline
\end{tabular}




\begin{tabular}{|c|c|}
\hline РуMT & Polyoma middle $\mathrm{T}$ virus \\
\hline RIN & RNA Integrity Number \\
\hline RIPA & Radio-Immunoprecipitation Assay \\
\hline RT & Room temperature \\
\hline SCID & Severe combined immunodeficiency \\
\hline SEM & Standard error of measurement \\
\hline SFE & Sphere formation efficiency \\
\hline STAT3 & Signal transducer and activator of transcription 3 \\
\hline STAT5 & Signal transducer and activator of transcription 5 \\
\hline TAD & Transactivation domain \\
\hline TCGA & The Cancer Genome Atlas \\
\hline TFM & Transcription factor matrix \\
\hline TIC & Tumor initiating cell \\
\hline TNBC & Triple negative breast cancer \\
\hline TRKB & Tropomyosin receptor kinase B \\
\hline TSS & Transcription start site \\
\hline ULA & Ultra low adhesion \\
\hline uPAR & Urokinase-type plasminogen-activator receptor \\
\hline UPL & Universal probe library \\
\hline VDR & Vitamin D receptor \\
\hline VEGF & Vascular endothelial growth factor \\
\hline VHL & von Hippel-Lindau \\
\hline WAP & Whey acidic protein \\
\hline
\end{tabular}


WCE

WISP2

WT
Whole cell extract

WNT1-inducible signaling pathway protein-2

Wild-type 


\section{CHAPTER 1. INTRODUCTION}

\section{Oxygen and Hypoxia}

Nearly all organisms have a constant requirement for oxygen $\left(\mathrm{O}_{2}\right)$. Normoxia is defined as the level of oxygen required for normal physiologic processes to occur, whereas low or insufficient levels of $\mathrm{O}_{2}$ is known as hypoxia. Oxygen deprivation will cause death faster than any other organic substance. Mammals in particular have evolved to have a complex respiratory and circulatory system to ensure sufficient delivery of $\mathrm{O}_{2}$ to the body in order to maintain homeostasis. In diseases that deprive cells of $\mathrm{O}_{2}$, such as heart attack and stroke, these systems are compromised resulting in dysfunctional enzyme activities, mitochondrial function, cytoskeletal structure, membrane transport and antioxidant defenses, ultimately leading to senescence, autophagy or cell death [2]. In cancer, tumor cells adapt to hypoxia, which can stimulate tumor growth, invasion, and metastasis [3]. General hypoxia occurs when the body as a whole is effected, whereas regional hypoxia may be confined to one specific area of the body. Hypoxia can also be heterogeneous within tissues, as occurs in cancer [4]. Additionally, hypoxic exposure may be acute, lasting a short time, or chronic, lasting longer periods of time and can be recurring [5]. In solid tumors, cells are often exposed to an $\mathrm{O}_{2}$ gradient that decreases gradually from efficient oxygenation to near anoxia. Moreover, tumor cells can undergo repeating cycles of $\mathrm{O}_{2}$ deprivation and reoxygenation [4].

Due to their rapid growth and progression, tumors exceed available $\mathrm{O}_{2}$ and nutrients, and are therefore exposed to low $\mathrm{pH}$, low glucose, and decreased oxygen. In order to adapt to this microenvironment and to continue expanding, tumors must bypass the normal controls regulating angiogenesis, cellular metabolism and the balance between survival or cell death [6]. The transcriptional response to hypoxia, known as the hypoxic response, is primarily regulated by the Hypoxia Inducible Factor (HIF) transcription factors [7].

\section{Oxygen and Hypoxia: Effects on Cancer Therapy}

It has been known for decades that hypoxic cells are resistant to killing by ionizing radiation [8]. Radiation damage produces a radical on DNA (DNA $\bullet$ ) molecules. This radical then enters into competition for oxidation by $\mathrm{O}_{2}$ to become permanent. In the absence of oxygen, this radical becomes reduced and the DNA returns to its original form [8]. Therefore, DNA damage is attenuated in the absence of oxygen. As most solid tumors have heterogeneous levels of hypoxia, with some regions having nearly no $\mathrm{O}_{2}$, they are often treated unsuccessfully with radiotherapy [3,9]. In addition to resistance to radiation, there have been many studies that demonstrate that hypoxic tumor cells are also resistant to chemotherapeutic agents. For example, hypoxic regions of tumors are often distant from blood vessels that carry anticancer agents through the mass. Therefore, hypoxic regions of the tumor may not be exposed to anticancer drugs [10, 11]. Additionally, hypoxia selects for cells that have lost sensitivity to p53-mediated apoptosis 
resulting in decreased sensitivity to apoptosis inducing chemotherapies [12]. Also of interest, hypoxia upregulates genes involved in drug resistance such as members of membrane protein families responsible for pumping drugs out of the cell, including $\mathrm{p}$ glycoprotein and breast carrier resistance protein-1 (BCRP1) [13, 14]. Hence, hypoxia exerts a negative influence on survival from cancer. Therefore, developing strategies that can overcome or bypass hypoxia-mediated therapeutic resistance is an important research field in solid tumorigenesis.

In particular, HIF-1 has been an attractive target for cancer therapies because it is a master transcriptional regulator of the hypoxic response and plays a key role in promoting angiogenesis, glucose utilization and tumor-cell survival in addition to its direct association with poor prognosis. Many pharmacological inhibitors of the HIFs and HIF-associated pathways have been developed and tested for the ability to prevent tumor growth in animal models, as reviewed in [15]. Yet, more research is needed to understand the functional relevance of HIF biology, and the role of HIF target genes and HIFdependent pathways during cancer progression and metastasis. Further elucidation of pathways and target genes downstream of the hypoxic response will provide valuable insight into the extensive role the HIF transcription factors play in tumor development, and may also reveal novel HIF-dependent genes that can be targeted for more effective cancer therapies.

\section{Regulation of Hypoxia Inducible Factors (HIFs)}

The HIFs bind DNA as a heterodimer composed of two basic helix-loop-helix (bHLH) proteins, the constitutively expressed aryl hydrocarbon nuclear translocator (ARNT/HIF- $\beta$ ), and its oxygen-responsive partner, the HIF-alpha subunits, HIF-1 $\alpha$ or HIF-2 $\alpha$ (Figure 1-1). Stability of the oxygen-responsive alpha subunits is tightly regulated at the post-translational level via interactions with the tumor suppressor protein von Hippel-Lindau (VHL) $[16,17]$. VHL provides substrate specificity to a large elongin $\mathrm{B} / \mathrm{C}$, ring-box 1 (RBX1), cullin2 (CUL2) complex that functions as an E3 ubiquitin ligase to target proteins for poly-ubiquitinylation and degradation by the $26 \mathrm{~S}$ proteasome [18]. Under normoxic conditions, HIF- $1 \alpha$ is hydroxylated on 2 evolutionary conserved proline residues (Pro-402 and Pro-564 in humans) by a family of prolyl 4-hydroxylases (PHDs) that belong to the 2-oxoglutarate-dependent oxygenase superfamily [19, 20]. PHDs depend not only on $\mathrm{O}_{2}$ but also on iron and 2-oxoglutarate. These two hydroxyl groups are necessary for VHL binding to the HIF-alpha subunits, which then mediates their rapid destruction [18]. In contrast, in response to decreasing oxygen, PHD activity decreases; therefore, HIF- $1 \alpha$ and HIF-2 $\alpha$ are stabilized, facilitating their dimerization with the constitutively expressed ARNT/HIF- $\beta$ to form the HIF-1 or HIF-2 transcription factor complexes. Increased protein stability results in the accumulation of nuclear HIF-1 or HIF-2 and coincides with a large and sustained increase in the transcription of genes that contain HIF binding elements (hypoxia response elements, HREs) in their regulatory regions. Microarray profiling has demonstrated that the HIFs directly stimulate or repress transcription of over one hundred genes in breast, renal, endothelial or embryonic fibroblast cells as part of the hypoxic response [21] [22, 23]. These target genes are 

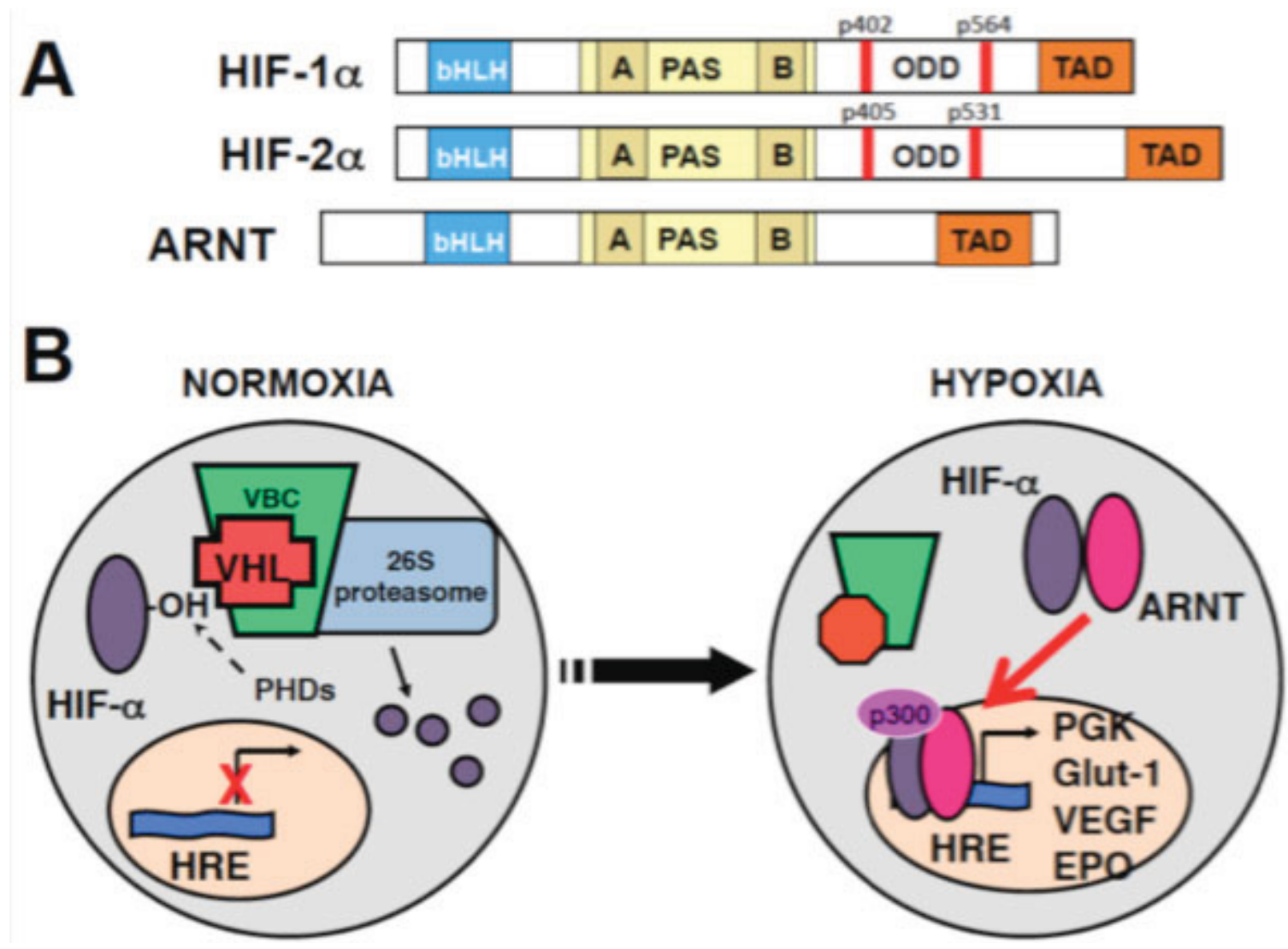

Figure 1-1. HIF protein structure and regulation.

(A) The protein general structure of HIF-1 $\alpha$ and HIF-2 $\alpha$ is highly conserved. Both HIF $\alpha$ subunits contain a basic helix-loop-helix domain (bHLH), a Per-Arnt-Sim (PAS) domain, an oxygen-dependent degradation domain (ODD), and a C-terminal transactivation domain (TAD). The HIF $\alpha$ binding protein, ARNT (HIF-1 $\beta$ ), does not contain the ODD. (B) Under normoxia, HIF $\alpha$ subunits are targeted for degradation via the hydroxylation of two key proline residues (red lines) in the ODD domain by the PHD enzymes, facilitating interaction with the VHL protein. At hypoxia, HIF $\alpha$ subunits are stabilized and bind to their shared partner ARNT to form the HIF-1 or HIF-2 transcriptional complexes, which mediate transcription of downstream target genes by binding to the hypoxic response element (HRE) within their promoters. 
implicated in control of a variety of cellular processes, including glucose transport, glycolysis, angiogenesis, cell cycle, chemotherapy and radiation resistance, invasion and metastasis, stem cell maintenance, stress response and apoptosis [24, 25]. While many of these genes can be regulated by either HIF-1 or HIF-2, some genes rely more heavily on one HIF $\alpha$ subunit or the other. For example the genes carbonic anhydrase $9(C A 9)$ and BCL2/adenovirus E1B $19 \mathrm{kDa}$ protein-interacting protein 3 (BNIP3) are more dependent upon HIF-1 activity, whereas epidermal growth factor $(E G F R)$ and WNT1-inducible signaling pathway protein-2 (WISP2) depend more on HIF-2 regulated transcription [26]. In addition to canonical HIF target mRNAs, the HIFs are also known to be involved in microRNA (miRNA) regulation [27]. For example, the microRNA, miR-210, which has been shown to be both hypoxia-inducible and a direct HIF-1 transcriptional target [28] is inversely correlated with disease-free and overall survival in breast cancer patients [29].

\section{HIFs Role in Normal Development}

$H I F 1 A$ and $H I F 2 A$ mRNAs are expressed in all mammalian tissues [30, 31]. However, in contrast to HIF1A, HIF2A mRNA is more highly expressed in developing vascular endothelium, fetal lung, and catecholamine-producing cells [32-37]. Several studies conducted in genetically modified mouse models have clearly demonstrated that deletion of either HIF gene is deleterious to development. One of the first indications that hypoxia-induced gene expression is a critical regulator of mammalian development was observed following the genetic deletion of the HIF $\alpha$ binding partner, ARNT (HIF-1 $\beta$ )[38, 39]. Arnt $^{-1-}$ mice died midway through gestation due to impaired blood vessel formation. In normal embryos, Hifla expression increases between E8.5 and E9.5, and Hifla ${ }^{-/-}$ global knockout (KO) embryos die by E11 [40, 41]. In HIF-1 $\alpha$ null mice, from E8.5 there was lack of blood vessel formation in the brain, along with a dramatic reduction in the number of somites and defective formation of the neural fold. In addition, these embryos exhibited multiple defects in cardiovascular development. Use of the nitroimidazole marker, EF5, which detects DNA and protein adducts in hypoxic cells, demonstrated global hypoxia in $\mathrm{Hifla}^{-/-}$embryos [41] as compared to wild-type (WT) littermates. Therefore, HIF-1 $\alpha$ is necessary for embryonic development.

Unlike Hifla, Hif2a seems to be more sensitive to the presence of modifier alleles in the various distinct mouse backgrounds used to generate the knockouts. As a result, independent groups have reported different phenotypes. One study observed embryonic lethality at mid-gestation as a result of a defect in catecholamine synthesis upon Hif2a deletion [36]. Another group targeting Hif2a null mice observed embryonic lethality between E9.5 and 13.5. They found that post-vasculogenesis changes required for the formation of the adult vasculature did not take place in these mice [42]. A third group reported that $H i f 2 a^{--}$embryos do not survive beyond E16.5, and while they had normal systemic vasculature, they experienced impaired fetal lung maturation [43].

Due to the lethality of global HIF $\alpha$ knockouts, conditional deletion of either Hifla or Hif2a, using Cre/lox technology, has become a widespread useful tool in studying HIF $\alpha$ knockout phenotypes in adult mice in specific tissues of interest [44, 45]. 
Investigators approach conditional knockouts by creating a "floxed" exon or promoter within a target gene by inserting loxP recombination sites in flanking introns or noncoding regions. Mice with "floxed" genes are normal and display no phenotype compared to WT controls. Floxed mice can then be bred with transgenic mice that express the Cre recombinase enzyme, which may be driven by a tissue-specific promoter. When the tissue-specific promoter is active, the Cre enzyme will excise the target sequence between the loxP sites thus creating a tissue-specific, conditional knockout of a gene. This approach has been used to study the HIF $\alpha$ family members and related HIFdependent proteins in many tissue types of the post-natal mouse.

As one example, Cramer et al observed that conditional deletion of Hifla in myeloid cells led to an unexpected suppression of macrophage and neutrophil function [46]. Another group found that conditional deletion of Hifla in skeletal muscle resulted in mice that had increased exercise endurance, but at the cost of increased muscle damage after repeated exercise bouts [47]. Conditional deletion of Hif2a 1 week after birth using a tamoxifen-inducible Cre system resulted in anemic mice [48]. Most closely related to our studies, the Johnson laboratory has deleted Hifla in mammary epithelial cells (MECs), and has shown that deletion of Hifla results in a failure of the mammary gland to functionally differentiate and to secrete milk proteins and lipids leading to impaired lactation $[49,50]$. These data, along with other defects observed in heart, colon, skin and brain [51-54] indicate that the HIFs are essential for normal development. Therefore, the expression levels of the individual $\mathrm{HIF} \alpha$ subunits must be tightly regulated since either their deletion or over-expression is detrimental to normal development and tissue physiology.

\section{HIFs Role in Tumorigenesis}

The biological function of the HIF pathway is highly relevant to tumorigenesis. HIFs play key roles in many critical aspects of cancer biology including angiogenesis [55-57], stem cell maintenance [58-60], metabolic reprogramming [61, 62]; autocrine growth factor signaling [63]; epithelial-mesenchymal transition [64-67], invasion [68, 69], metastasis [70-72], and resistance to radiation therapy [73] and chemotherapy [74]. Overexpression of HIF- $1 \alpha$ and HIF- $2 \alpha$ has been documented in multiple human cancers, including breast cancer $[75,76]$. In particular over-expression of HIF-1 $\alpha$ protein has been observed in many tumor metastases, including breast cancer [77]. Immunohistochemical analyses demonstrated that there are detectable levels of HIF-1 $\alpha$ protein in benign breast lesions, elevated levels in primary poorly differentiated tumors, and a marked further increase in tumor metastases, in contrast to virtual absence of HIF- $1 \alpha$ staining in normal tissues $[76,78]$. In a model of Ras-transformed, immortalized mouse embryonic fibroblasts (MEFs), injection of HIF-1 $\alpha$ (or HIF-1 $\beta$ ) wild-type or knockout cells to immunocompromised mice revealed that HIF-1 $\alpha$ is a positive factor during tumorigenesis $[49,79]$. In addition to the response to a hypoxic microenvironment, HIF $\alpha$ expression can be further enhanced by cancer-causing mutations. For example, loss of wild-type tumor suppressor p53, loss of VHL, loss of PTEN and HER2 amplification all result in either increased HIF protein expression or HIF-dependent transcription [80-82]. 
Moreover, HIF levels can be activated by growth factor stimulation in an oxygenindependent manner through the phosphatidylinositol 3-kinase (PIK3) or mitogenactivated protein kinase (MAPK) pathways [24].

\section{HIFs Role in Breast Cancer}

Breast cancer is the second most commonly diagnosed cancer, with 1 in 8 women developing breast cancer over their lifetime. Relapse due to metastatic lesions is the primary cause of breast cancer related deaths. Oxygen tensions in advanced breast cancers can be as low as $0.1-1 \% \mathrm{O}_{2}$ [83], a range commonly used to model tissue hypoxia in vitro. A majority of ductal carcinoma in situ (DCIS) and almost all poorly differentiated, invasive breast carcinomas over-express HIF-1 $\alpha$ [84]. HIF-1 $\alpha$ protein is over-expressed in $\sim 29 \%$ of primary breast tumors and $\sim 69 \%$ of metastases [76]. Either the over-expression of HIF-1 $\alpha$ protein or the enrichment of a hypoxic gene signature in the primary tumor correlates with poor prognosis and decreased survival in breast cancer patients $[21,85]$.

Breast tumors are diverse in their genetic make-up and individual responses to therapy. In 2000 Perou et al characterized gene expression patterns in a set of primary human breast tumors using DNA microarrays [86]. The expression patterns identified by the array provided a distinctive molecular portrait for each tumor. Based on these data, tumors could be classified into five intrinsic subtypes of breast cancer distinguished by differences in their gene expression patterns. The five subtypes identified are commonly known as Luminal A (ER ${ }^{+}$, typically low grade), Luminal $\mathrm{B}\left(\mathrm{ER}^{+}, \mathrm{PR}^{+}\right.$, typically high grade), HER2- enriched (amplified for HER2 protein), Normal-like and Basal-Like (ER, $\mathrm{PR}$, and HER2 negative) $[86,87]$.

Based on hierarchical clustering, the basal-like subtype is characterized by high expression of various markers of the basal or myoepithelial cell linages, such as the keratins 5 and 17, laminin and fatty acid binding protein. In addition, the basal-like tumors predominantly include tumors negative for the steroid receptors ER, PR, and HER2, hence they are commonly referred to as triple negative breast cancers (TNBC, Figure 1-2) [86, 87]. TNBCs tend to have the worst prognosis, as they rapidly develop resistance to conventional chemotherapies, including the frontline therapy doxorubicin (DOX), and patients are at a high risk for recurrence and decreased survival $[88,89]$. The HER2-enriched tumors are characterized by high expression of several genes in the ERBB2 amplicon, including ERBB2 and GRB7. Recent studies from our lab have shown that HIF- $1 \alpha$ protein is highly expressed in HER $2 / \mathrm{Neu}$ positive murine mammary tumors and that HIF- $1 \alpha$ is required to promote anoikis survival, a feature required for cells to acquire full metastatic potential [90]. Tumor samples in the normal-like group show the highest expression of many genes known to be expressed by adipose tissue and other nonepithelial cell types. In contrast, the luminal subtypes are characterized by high expression markers of the epithelial cell lineage, such as keratins 8 and 18, as well as high expression of estrogen receptor alpha (ESR1/ER $\alpha$ ). These tumors, therefore, can be targeted with anti-estrogenic therapies, and patients tend to have better prognosis and 

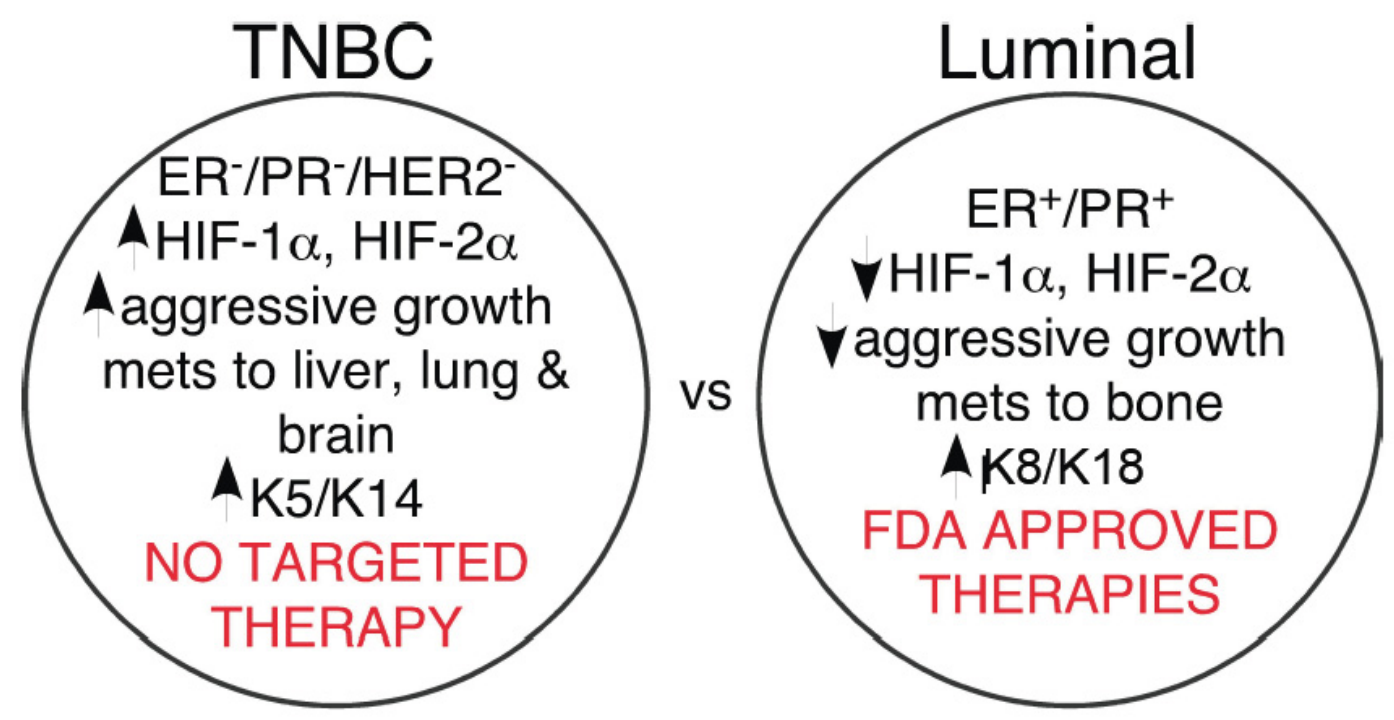

Figure 1-2. Overview of differences between triple negative and luminal breast cancer.

Basal breast cancers, including triple negative breast cancers (TNBC), are generally ER/PR/HER2 negative and express high levels of HIF $\alpha$ proteins. Additionally, these tumors exhibit aggressive growth with metastases in the liver, lung, and brain and no FDA-approved targeted therapies are currently available. In contrast, luminal tumors are generally ER/PR positive and express lower levels of the HIF $\alpha$ proteins [1]. Luminal tumors are typically less aggressive and metastasize mainly to bone. There are many FDA approved therapies for luminal tumors. 
longer survival times [91]. Whole genome expression profiling of the breast cancer subtypes has revealed that TNBCs and HER2-enriched tumors have an enriched hypoxic core gene signature as compared to luminal breast cancers [92]. Independently, the HIF gene signature was also identified as a major pathway enriched in TNBCs versus luminal cancers in The Cancer Genome Atlas Network (TCGA) study, which profiled over 800 primary samples of human breast cancer [93].

Although HIF-1 $\alpha$ was previously hypothesized to be the predominant regulator of the hypoxic response in breast cancer cells $[1,22,26]$, emerging evidence also indicates that HIF-2 $\alpha$ may also play a role in breast cancer growth, as will be further discussed in Chapter 4. Recent studies have proposed that while HIF-1 controls the acute or immediate response to hypoxia during tumor development, HIF-2 may be responsible for the prolonged hypoxic response [26]. Furthermore, there is evidence indicating the role that HIF-1 or HIF-2 plays within tumors depends on their opposing regulation of MYC transcriptional activity [94]. In addition to the differing roles each HIF $\alpha$ isoform plays in tumor progression, there have been conflicting reports on the prognostic value of HIF- $2 \alpha$. One study found HIF-2 $\alpha$ associated with distant recurrence and poor outcome in invasive breast cancer [95], while another reported significantly longer patient survival in breast cancer with high levels of HIF-2 $\alpha$ [26]. It is likely that both HIF-1 and HIF-2 each play important, and perhaps opposing roles, in the development of breast cancer and the contribution of each HIF $\alpha$ subunit in the various subtypes of breast cancer must be better understood for the development of successful targeted therapies against the HIF pathway.

\section{Significance of the Hypoxic Response in Metastasis}

The ultimate mortality of patients with solid tumors results from the occurrence of distance metastases. Metastasis is the outcome of processes that involve interactions between cancer cells and their microenvironment to allow tumor cells to populate and colonize in new tissue habitats and, ultimately, cause organ dysfunction and death. The basic steps of metastasis include 1) local invasion of tumor cells into surrounding stroma, 2) intravasation, cells entering into the blood stream, 3) circulation, 4) extravasion, exit of tumor cells from circulation into a new organ and 5) colony formation of cells at distant organ sites. All of these steps require the coordination of numerous genes, many of which are regulated by the HIF transcription factors [96]. The first step in local invasion requires basement membrane disruption, and this process is promoted by the HIF-1 $\alpha$ dependent upregulation of cathepsin D (CTSD), urokinase-type plasminogen-activator receptor (uPAR), and matrix metalloproteinase-2 (MMP2) [97]. Hypoxia also upregulates expression of lysyl oxidase (LOX) to increase focal adhesion, cell migration and motility [70]. The HIF-1 $\alpha$ target proteins matrix metalloproteinase 1 (MMP1) and MMP2 are synergistic mediators of vascular permeability and the intravasation step [98] and the hypoxia responsive factor TRKB, a suppressor of anoikis, may be responsible for keeping cells alive during circulation [99, 100]. Extravasion is promoted in a similar way to intravasation, by the HIF-1 $\alpha$ target genes VEGF, MMP1 and MMP2. Furthermore, angiopoetin like 4 (ANGPTL4), another direct HIF-1 $\alpha$ target gene, is responsible for extravasion of breast tumor cells to the lung [72]. Homing of tumor cells to distant organs 
is responsible in part by chemokine receptor CRCX4, a direct HIF target gene. Once cells have extravasated in the organ, tumor cell outgrowth is also a hypoxia-mediated event involving genes such as connective tissue growth factor (CTGF), interleukin 6 (IL6), interleukin 8 (IL8), vascular endothelial growth factor (VEGF), osteopontin (OPN) and many others [101].

Although significant progress has been made in early diagnosis of breast cancer and the treatment of hormone-receptor positive ("luminal") and HER2+ breast cancers, the overall survival rate of patients with metastatic breast cancer (MBC) remains dismal. Since breast cancer deaths are due to metastatic disease, basic research need to focus more on identifying pathways that promote metastasis, and not just regulate primary tumor growth. The hypoxic response is clinically relevant to MBC since hypoxic tumor cells are refractory to chemotherapy and radiation and since hypoxia stimulates cell migration and metastasis $[25,102]$.

\section{Identification of Novel or Understudied HIF-Regulated Genes to Identify New Therapeutic Targets in Breast Cancer}

As I will discuss further in Chapter 3, when Hifla is conditionally deleted in the mouse MMTV- PyMT transgenic model of breast cancer, there is a delay in tumor latency and a dramatic reduction in lung metastases. These studies confirm that HIF-1 $\alpha$ has a direct role in both breast cancer progression and metastasis. Therefore, strategies that down-regulate HIF-1 $\alpha$, or its downstream target genes, may be effective in enhancing clinical outcome. To better understand how hypoxia drives not only breast cancer progression, but also breast cancer metastasis, our lab performed whole-genome mRNA microarray profiling. We identified 94 mRNAs that are commonly differentiallyexpressed in each of two settings: 1) WT and KO cells cultured at hypoxia ( $6 \mathrm{~h}, 0.5 \% \mathrm{O} 2)$ and 2) in end-stage mammary tumors derived from transplantation of parental WT and KO cells, with 68 mRNAs being up-regulated and 26 mRNAs being down-regulated. Several of these genes were identified that regulate cell proliferation and/or the metastatic switch, in additional to multiple genes involved in stem cell maintenance. In Chapter 5, which reviews data generated from the whole genome screen, I will discuss some of these genes in further detail. In addition, in Chapter 6, I will further focus on one gene we found to be particularly relevant to breast cancer biology, creatine kinase brain isoform $(C k b)$.

There are currently a number of different HIF inhibitors being used in small animal studies and clinical trials, as reviewed in [24, 103]. While some have shown initial success in delaying tumor growth and prolonging survival, such as digoxin [104], YC-1 $[105,106]$ and PX-478 [107], the most effective therapies have been those that target HIF-related pathway genes. For example, Traztuzumab (Herceptin) is a frontline therapy in the treatment of patients with HER2-enriched breast cancers [80], and as previously described HIF- $1 \alpha$ is highly expressed in HER2 positive tumors. Additionally, mTOR, which regulates HIF-1 $\alpha$ translation initiation, can be inhibited by Rapamycin [108], another commonly used chemotherapy drug in the treatment of cancers, including breast 
cancer. Doxorubicin and daunorubin, two anthracyclines which have been reported in one study to inhibit HIF transcriptional activity [24] are also frontline therapies in the treatment of several solid tumors.

All of these drugs, although initially effective, do come with drawbacks and debilitating side effects. Moreover, most of these compounds treat only the recurrence of the primary tumor and do not focus on the prevention or treatment of MBC. Therefore, identifying novel or understudied HIF-dependent genes, such as those identified on our microarray, may lead to improved targeted therapies for the treatment of not only primary tumors, but also for the inhibition of metastasis, perhaps with decreased negative side effects, leading to increased patient survival.

\section{Hypoxia and Cancer Stem Cells}

\section{Cancer Stem Cell Theory and Regulation of Breast Cancer Stem Cell Activity by HIF}

Breast cancer cells exhibit properties of cancer stem-like cells (CSCs), also referred to as tumor initiating cells (TICs). The CSC hypothesis postulates that tumors arise from a small population of cancer cells with stem cell-like properties [109], with a corollary that CSC-like cells play a primary role in relapse promoting refractory disease and death due to metastases [110]. Hypoxic exposure promotes self-renewal of several cell types, including neurospheres, hemapoietic stem cell cells (HSCs) and embryonic stem (ES) cells [111]. HIF-1 is also necessary in vivo for HSCs since its deletion causes stem cell exhaustion [112]. In addition, hypoxic regions of secondary breast tumors that arise after treatment of the primary tumor with anti-angiogenic therapies are enriched with CSC-like cells [113]. Another common feature of hypoxic cells and CSCs is that both hypoxic regions of tumors and CSC-like cells are known to be highly refractory to radiation and chemotherapy $[102,114]$.

Accumulating evidence supports the hypothesis that stem cells and TICs exist in a hypoxic niche microenvironment [111]. The direct relationship of the hypoxic response to TIC activity has been demonstrated in adult glioma, human acute myeloid leukemia (AML) and murine lymphoma [60, 115]. In gliomas, expression of HIF2A was enriched in CSCs, and knockdown of $H I F 2 A$, but not $H I F 1 A$, reduced TIC activity in cells derived from patient xenografts [115]. In contrast, HIF-1 was found to be essential for maintaining TIC activity in a syngeneic rodent transplant model of lymphoma and in AML patient xenografts via regulation of the Notch pathway [60]. Interestingly, in gliomas, the TIC population was enriched via cell sorting based on the expression of a single cell surface marker, CD133 (PROM1). PROM1, a transmembrane protein without a known ligand, is a known hypoxia-responsive protein regulated by HIF-1 [116, 117]. Several studies have shown that the population of breast tumor cells with the ability to self-renew is enriched with the ability to initiate tumorigenesis in vivo [118-121]. Furthermore, TICs may drive metastasis $[122,123]$. 


\section{HIF Regulation of Cancer Stem Cells in the MMTV-PyMT Mouse Model}

The specific contribution of HIF- $1 \alpha$ or HIF- $2 \alpha$ in regulating TIC activity in breast cancer is still being defined, particularly in the context of syngeneic rodent models that recapitulate the breast cancer microenvironment. The MMTV-PyMT model is one of the most commonly utilized pre-clinical mouse models in breast cancer research since tumor latency is short and there is a high frequency of metastasis to the lung [124]. Wholegenome array profiling indicates that PyMT tumors most closely resemble the luminal B subtype of human breast cancer [125], although end-stage PyMT tumors are estrogen and progesterone receptor (ER/PR)-negative [124]. Dr. Seagroves has previously shown that conditional deletion of Hifla in PyMT+ tumors by crosses to mice expressing MMTVCre recombinase delayed the onset of palpable tumors, delayed the progression of hyperplasias to carcinomas and reduced lung metastases [126]. Our lab created in an improved model system in which Hifla is efficiently deleted in the mammary tumor epithelium via ex vivo viral transduction with Cre recombinase prior to the injection of mammary tumor cells to the cleared fat pads of recipient mice [127]. Validating the use of a transplantation paradigm in lieu of intact transgenic mice, PyMT tumor cells transplanted serially into recipients exhibit similar morphology and gene expression profiles as the original tumors [128].

As I will review in further detail in Chapter 3, using these novel HIF-1 $\alpha$ wild type (WT) and null (KO) mammary tumor epithelial cells (MTECs), we demonstrated that HIF-1 $\alpha$ prominently augments primary tumor growth and lung metastasis and accelerates relapse due to metastasis through regulation of breast CSC activity. Furthermore, we demonstrated for the first time that HIF-1 $\alpha$ promotes mammary tumorsphere formation and enhances TIC frequency in vivo, in part through regulation of the expression of markers associated with the basal lineage, the Notch pathway and CD133. Together, these data indicated that suppressing the hypoxic response may be beneficial not only to reduce primary tumor mass but also to suppress the breast TIC sub-population that may be ultimately responsible for patient relapse. Through our studies investigating the expression of several cell surface markers in WT and KO PyMT cells and tumors, we observed that several cell surface proteins routinely used to enrich cells for CSC activity were up-regulated by HIF-1. One set of results from these studies, focusing on HIFdependent regulation of CD49f, will be further discussed in Chapter 4.

\section{Moving into Translational Research: Investigating the Role of HIF-1 and HIF-2 in Human Models of Luminal and Triple Negative Breast Cancers}

Traditionally, cancer research has focused on in vitro characterization of conventional human cell lines derived from patient samples. In the 1990's several genetically modified syngeneic mouse models were created that represented an improvement in modeling breast cancer [129]. While these models have provided valuable insight into cancer biology, they still face some limitations. One of the main drawbacks in using genetically modified mouse models is that, as a result of originating from a murine background, tumors do not always replicate the histology, physiological 
effects, biochemical pathways or metastatic pattern observed in the corresponding human tumor type $[129,130]$. Additionally, the use of conventional human breast cancer cell lines cultured in monolayer in the presence of serum only partially replicates the genetic features and metastatic potential of tumors observed clinically in patients [131]. These limitations make it difficult to accurately predict patient responses to therapy. Recently advances have been made in patient derived mouse xenograft models that more faithfully replicate clinical phenotypes. For example, Dr. Alana Welm at The Huntsman Cancer Institute (HCI) has developed a new model of serially transplantable, orthotopic breast tumor grafts [132] that represents the next major advance in modeling metastatic breast cancers in mice. In addition, Zhang and colleagues [133] and Kabos and colleagues [134] have had success in establishing patient derived xenografts (PDXs), as reviewed in Landis et al [135].

To create the HCI breast tumor tissue bank, the Welm laboratory transplanted fresh primary tumor material, metastatic breast cancer samples or freshly collected tumor cells present in pleural fluid into female NOD/SCID mice immediately following harvest [132]. As of 2011, thirteen tumor lines from 10 patients were successfully maintained through multiple rounds of serial transplantation. Being serially passaged only through mice, these tumors were never exposed to in vitro cell culture conditions and manipulations, and, therefore maintained their gene expression and SNP profiles over sequential generations, and recapitulated the same sites of metastasis in the mouse from the primary tumor as was clinically observed in the patients. The tumors engrafted represent a variety of breast cancer subtypes including 6 luminal tumors, 1 HER2enriched tumor, and 5 basal-like tumors [132]. All tumor grafts were evaluated by pathologists following 4-8 rounds of serial transplantation, and all retained the key characteristics of the original tumors. The most striking feature of these tumor grafts was their ability to spontaneously metastasize from the orthotopic site with the same patterns of metastasis as seen in the original patient. Further evaluation using molecular gene signatures demonstrated that tumor grafts clustered with their original human tumor and within a node containing other primary tumors of their subtype. The features of this model provide a unique way in which to study breast cancer and offer multiple advantages over breast cancer cell lines including (1) the ability of grafts to maintain critical features of the original tumors including histopathology, clinical markers, and gene expression profiles; (2) the representation of all major clinical types of breast cancer; and (3) the spontaneous metastasis of grafts to many of the same organs that were affected in patients.

Use of the HCI tissue bank will be useful in our lab to explore the differing roles that the HIFs may play in different subtypes of breast cancer. For example TNBCs and HER2-enriched tumors have relatively high levels of both HIF-1 $\alpha$ and HIF- $2 \alpha$ compared to luminal tumors $[1,92]$, and this could confer to them more rapid and aggressive growth. As I will discuss in greater detail in Chapter 6, by knocking down individual HIF $\alpha$ subunits in specific tumor subtypes, we will be poised to evaluate how deletion of each HIF $\alpha$ gene impacts primary tumor growth and metastases. Moreover, labeling the tumor cells with a reporter, such as firefly luciferase (Luc2), will facilitate live bioimaging over time. Using these tools and techniques, we will have the ability to assess 
how rapidly tumors and their metastases form in the presence or absence of HIF $1 A$, $H I F 2 A$ or both genes, even before we are able to manually measure any tumor mass with calipers. Finally, we plan to use this resource to study novel HIF-dependent genes, originally identified in the MMTV-PyMT transgenic mouse in a more clinically relevant human model to propose of testing novel, targeted HIF therapies that may be beneficial in the clinic to prevent MBC or to prolong overall survival (OS).

\section{Hypothesis and Specific Aims}

Our hypothesis is that there is a subset of novel or understudied HIF- $1 \alpha-$ dependent genes in mammary tumor epithelial cells (MTECs) that primarily contribute to tumor growth and/or metastasis. Additionally, there is a population of tumor-initiating cells with stem-like features that require the HIF-1 or HIF-2 transcription factors to promote tumor initiation, growth, metastasis and chemoresistance. Two aims were designed to investigate this hypothesis:

- To investigate the contribution to tumor growth and metastasis of the most highly HIF-1-dependent genes with a prior implicated role in cancer.

- To delineate the role of HIF regulation of tumor initiating cells (TICs) and how TICs may influence therapeutic resistance in human breast cancers. 


\title{
CHAPTER 2. VHL DELETION IMPAIRS MAMMARY ALVEOLOGENESIS BUT IS NOT SUFFICIENT FOR MAMMARY TUMORIGENESIS ${ }^{\dagger}$
}

\section{Introduction}

Hypoxia-Inducible Factor-1 (HIF-1) $\alpha$ is over-expressed in most solid tumors and correlates with poor prognosis and high risk of metastasis in breast cancer patients. Because HIF-1 $\alpha$ protein stability is tightly controlled by its interaction with the tumor suppressor protein von Hippel- Lindau (VHL), deletion of $V H L$ results in inappropriate, constitutive HIF-1 $\alpha$ expression. In order to determine if $V H L$ plays a role in normal breast development, and if HIF-1 $\alpha$ over-expression is sufficient to initiate breast cancer, we conditionally deleted Vhlh (the murine $V H L$ homologue) in the mammary epithelium using the Cre/lox system. Upon first pregnancy and lactation, loss of Vhlh produced subtle changes in epithelial cell differentiation. However, $V h l h^{-/-}$mammary glands from multiparous mice exhibited a progressive loss of alveoli with each pregnancy/lactation cycle and the few alveoli that formed were poorly differentiated. Deletion of $V h l h$ in the epithelium also impacted the mammary stroma, as indicated by increased microvessel density, accompanied by areas of hemorrhage. However, deletion of $V h l h$, and therefore, constitutive Hif- $1 \alpha$ expression, was not sufficient to induce mammary tumorigenesis, consistent with a lack of report of increased breast cancer risk in patients with VHL disease. Moreover, co-deletion of Hifla/Vhlh was not sufficient to rescue the $\mathrm{Vhlh}^{-/-}$ phenotype, suggesting that additional genes besides HIF-1 $\alpha$ are mis-regulated in response to deletion of Vhlh in the breast epithelium.

\section{Methods}

\begin{abstract}
Animals
Vhlh conditional mice (flanked by loxP sites, or "floxed") were obtained directly from Dr. Volker Haase and generated as described in [136]. Hifla conditional mice were generated as in [41, 49]. Mice expressing the MMTV-Cre (line D) or the Wap-Cre transgene were obtained from Dr. Kay-Uwe Wagner (University of Nebraska, Eppley Cancer Center) $[137,138]$. Female mice used in experiments were generated by mating floxed/floxed; MMTV - or Wap-Cre-positive male mice with floxed/floxed ("double floxed", DF); Cre-negative females, producing littermate control (Cre-negative) and test (Cre-positive) progeny. To co-delete Hifla and Vhlh, Vhlh/Hifla compound DF females were bred to $\mathrm{Vhl} / \mathrm{Hifla}$ floxed/floxed males positive for MMTV-Cre. Therefore, in all cases, expression of Cre would induce homozygous deletion of the floxed gene. Animals
\end{abstract}

\footnotetext{
${ }^{\dagger}$ Adapted with permission. Seagroves, T. N., Peacock, D.L, et al. (2010). "VHL deletion impairs mammary alveologenesis but is not sufficient for mammary tumorigenesis." Am J Pathol 176(5): 2269-2282.
} 
were housed in microisolator cages and provided food and water ad libitum in an Association for Assessment and Accreditation of Laboratory Animal Care (AALAC)approved facility under protocols approved by the University of California San Diego or the University of Tennessee Health Science Center (UTHSC) Laboratory Animal Use and Care Committee.

\section{Protein Extraction and Western Blotting}

Whole inguinal mammary glands were harvested from C57BL/6 female mice (JAX, Bar Harbor, ME) at the following developmental stages: mature virgin (12 wks of age), day 6 (6-P), 10 (10-P), 15 (15-P) and 18 (18-P) of pregnancy, based on the date of plug observed as day 0 , day 10 of lactation and at involution (following 4 days of forced weaning from day 10 of lactation); $n=3$ mice/time point. Using procedures previously described [139], tissue extracts were prepared from whole mammary glands for each time point from frozen, pooled tissue that was ground to a fine powder under liquid nitrogen and immediately homogenized in a modified RIPA buffer to produce a whole cell extract (WCE) comprised of cytoplasmic and nuclear proteins. WCE were resolved on 10\% BisTris NuPAGE gels (Invitrogen, Carlsbad, CA) and blotted to PVDF membrane (Millipore, Bellirica, MA). The membrane was blocked in 5\% non-fat dry milk prior to addition of anti-VHL antibody (clone Ig32, BD Biosciences, San Jose, CA) at 1:200 for $3 \mathrm{~h}$ at RT, followed by incubation in a 1;10,000 dilution of anti-mouse IgG-HRP (GE Life Sciences, Piscataway, NJ) for 30 minutes, and development in ECLPlus substrate (GE Life Sciences). Membranes were stripped and re-probed with an anti-guinea pig cytokeratin 8/18 primary antibody (Progen GmbH; Heidelberg, Germany) at 1:5,000 for $1 \mathrm{~h}$ at RT, followed by incubation in a 1:5,000 dilution of donkey anti-guinea pig IgGHRP (Jackson Immunologicals, West Grove, PA), prior to exposure to ECLPlus substrate.

\section{Continuous Breeding and Lactation Studies}

To examine the effects of $V h l h$ deletion in multiparous females, homozygous floxed, Cre-negative or Cre-positive females were housed singly and continuously bred to FVB males. During constitutive breeding, all pups were weaned at 21 days of age. For glands harvested during the lactation period, litter size was normalized on the date of birth to 8 pups per dam, using either the endogenous litters (born to the dam) or by crossfostering pups that were born to lactating FVB dams. To permit accumulation of milk in the lactating gland, all litters were weaned from nursing dams for 2-3h prior to gland harvest, and all pups then returned to the mother once she recovered from anesthesia.

\section{Tissue-Harvest, Histology and Whole-Slide Scanning}

Individual inguinal (\#4) or thoracic (\#3) mammary glands were biopsied from anesthetized mice at specified time points during the first pregnancy or lactation and/or 
during sequential survival surgeries performed on the same animal over the course of multiple gestations. Each gland was fixed in neutral phosphate-buffered formalin (NBF) for $6 \mathrm{~h}$ at room temperature (RT) and/or flash frozen in liquid nitrogen for preparation of RNA or protein. Fixed tissue was embedded in paraffin and $5 \mu \mathrm{m}$ sections prepared. Sections were stained with hematoxylin and eosin (H\&E) or used for immunohistochemistry.

\section{Immunohistochemistry}

Antigen retrieval, GLUT-1 and CD34 immunostaining and the calculation of microvessel density using Chalkley analysis were performed as previously described [50, 126]. To detect HIF-1 $\alpha$, sections were incubated with a 1:100 dilution of a rabbit polyclonal anti-human HIF-1 $\alpha$ antibody (generated to amino acids 600-800) generously provided by Dr. Robert Abraham, Burnham Institute, La Jolla, CA. Leukocytes were detected with the pan-leukocyte marker CD45 (BD Biosciences) at a 1:100 dilution. VEGF was detected with a 1:40 dilution of a goat polyclonal antibody to murine VEGF (R\&D Biosystems, Minneapolis, MN). The Vector ABC Elite staining kit (Vector Laboratories, Burlingame, CA) was utilized in conjunction with DAB (3,3'diaminobenzidine) or DAB Impact peroxidase reagent (Vector Laboratories) to visualize all immunoreactive complexes. All slides were counterstained with Harris hematoxylin. To determine the percentage of proliferating mammary epithelial cells (MEC) during pregnancy, $15-\mathrm{P}$ females were injected $2 \mathrm{~h}$ prior to tissue harvest with $0.1 \mathrm{~mL} / 10 \mathrm{~g}$ body weight of cell proliferation reagent (Bromodeoxyuridine, BrdU, GE LifeSciences). BrdU incorporation was detected by anti-BrdU-FITC antibody (BD Biosciences, San Jose, CA) and the average percentage of $\mathrm{BrdU}+$ cells/MEC ( $\mathrm{n}=5$ animals/genotype) was determined as previously described [140]. Similar procedures were utilized to calculate the percentage of CD45+ cells/total MEC in glands harvested from multiparous dams, except that 5 random fields at $40 \mathrm{x}$ magnification were observed ( $\mathrm{n}=4$ animals/genotype). To visualize the gross vasculature of the mammary gland, thrice parous lactating dams were anesthetized, the thoracic mammary gland was bathed in PBS and the glands were digitally imaged under a Leica dissecting stereozoom microscope. Dams were then returned to their respective weaned litters following recovery from surgery.

\section{Automated Analysis of Epithelial Cell Content}

The Aperio Pixel Density algorithm was utilized to quantitate the number of MEC contained within inguinal, $\mathrm{H} \& \mathrm{E}$-stained glands harvested from mice at $15-\mathrm{P}(\mathrm{n}=4$ mice/genotype) or 18-P ( $\mathrm{n}=3$ mice/genotype). Default settings for this algorithm were adjusted until pixels from the stroma and the vasculature were not detected. The following settings were utilized: Image Zoom, 1; Mark-up Compression, 0; Compression Quality, 30; Classifier Neighborhood, 30; Classifier, 30; Hue Value, 0.75; Hue Width, 0.35; Color Saturation Threshold, 0.25; Iwp (high), 200; Iwp (low), 175; Ip (low), 100, Isp (low); Inp (high), -1 . The total number of positive pixels was calculated by adding the weak, median and strongly positive pixel values (Nweak, Np and Npositive, respectively) 
and dividing this total by the area analyzed (in $\mathrm{mm}^{2}$ ). The average number of positive pixels $/ \mathrm{mm}^{2}$ was then compared between genotypes. In all cases, the area occupied by the inguinal gland lymph node was excluded.

\section{Gene Expression Analysis by RT-PCR}

Total RNA was prepared from flash frozen whole, inguinal mammary glands harvested at 18-P or on the date of birth during the first lactation. Tissue was ground to a fine powder under liquid nitrogen and homogenized immediately in RNABee reagent (Isotex Diagnostics, Friendswood, TX). Total RNA was prepared according to manufacturer instructions and RNA quality was analyzed by the Agilent 2100 Bioanalyzer (Santa Clara, CA). Only samples with RNA Integrity Number (RIN) values higher than 9.0 were chosen for preparation of cDNA using the High-Capacity cDNA Reverse Transcription kit (ABI, Foster City, CA). Real-time PCR was performed using optimized primer and FAM-labeled probe sets designed by the Roche Universal Probe Library Assay Design Center software available on the Roche website. Real-time PCR was performed using the Roche LightCycler 480 machine for 40 cycles. The crossing point $(\mathrm{Cp})$ for each sample was determined using the Roche absolute quantitation algorithm. The average sample $\mathrm{Cp}$ value was then calculated for all independent replicates/genotype/time point. To control for cDNA input, the average sample Cp values were first normalized based on expression of the Ints 3 gene. To compensate for changes in MEC content among genotypes, Ints3-normalized, average Cp values were then normalized for expression of cytokeratin 18 (Krt 18). The average fold-change (FC) in gene expression was determined by comparing the dual-normalized $\mathrm{Cp}$ values between control (floxed/floxed) and gene-deleted samples using the ${ }^{\Delta \Delta} \mathrm{Cp}$ method.

\section{Statistics}

Statistical significance was determined using an unpaired Student's $t$ test (Prism 4.0, GraphPad). All p-values less than 0.05 were considered to reflect statistically significant changes between genotypes.

\section{Results}

\section{VHL Expression Increases during Pregnancy and Decreases during Involution}

To determine the expression levels of VHL over the course of development of normal mammary gland, whole cell protein extracts (WCE) were prepared from whole mammary glands harvested from C57BL/6 nulliparous, pregnant, lactating or involuting females and VHL detected by western blotting. As a positive control, extract from a whole E15 embryo was included. A single isoform of VHL was detectable at $24 \mathrm{kDa}$ at all stages of development. Expression of VHL increased dramatically from early (6-P) to 
mid-pregnancy (10-P), a period of rapid alveolar expansion, and levels remained high until involution, a time of extensive epithelial cell death and tissue remodeling

(Figure 2-1A). Based on the increased expression of cytokeratins 8/18 over the course of mammary gland development, known markers of the luminal mammary epithelium, VHL expression appears to increase as the number of epithelial cells increases. These data suggest that murine VHL is preferentially expressed by mammary epithelial cells (MEC) relative to the mammary stroma, in agreement with prior reports for the human breast [141].

In order to specifically delete Vhlh in the mammary epithelium, a Cre/lox strategy was employed. Previously characterized Vhlh homozygous floxed mice were bred to either MMTV-Cre or WAP-Cre transgenic mice. Whereas in line D of MMTV-Cre mice the Cre transgene is activated upon puberty and induces recombination throughout mammary gland development [137], the Wap-Cre transgene is only induced at high levels beginning at mid-pregnancy [142]. Furthermore, there is promiscuous expression of Cre when its expression is driven by the MMTV promoter since recombination activity was observed in other tissues, including the skin, B and T cells and the pancreas. In contrast, expression of the Wap-Cre transgene is highly restricted to the mammary epithelium of pregnant and lactating mice [137].

To confirm that we successfully generated Vhlh conditional knockout mammary epithelium $\left(V h l h^{\mathrm{MEC}-/-}\right)$, WCE were prepared from whole mammary glands of agematched, littermate lactating dams that were either negative or positive for the Wap-Cre transgene and subjected to western blotting for VHL. VHL was barely detectable in extracts prepared from Wap-Cre-positive mice, confirming efficient deletion of $V h l h$. The blots were stripped and re-probed with antibodies to cytokeratin 8/18 (CK8/18)

(Figure 2-1B). The extent of deletion of VHL observed at lactation was similar between Wap-Cre and MMTV-Cre transgenic mice (data not shown).

\section{Conditional Deletion of Vhlh Impairs MEC Proliferation and Differentiation during Pregnancy}

We next assessed the contribution of Vhlh to mammary gland development by comparing the histology of wild type and $V h l h^{-/ \mathrm{MEC}}$ mammary glands in which deletion was achieved via expression of the MMTV-Cre (line D) transgene. In this particular line of MMTV-Cre transgenic mice, Cre is activated in epithelial cells at approximately 3 weeks of age, when ductal morphogenesis is in progress [137]. However, no gross differences in ductal branching or extent of outgrowth into the mammary fat pad were observed in mature nulliparous mice that had completed ductal morphogenesis Likewise, no gross changes were observed in alveolar number or appearance at day 6 of pregnancy (early pregnancy) or at day 10 of pregnancy (mid-pregnancy) [143].

The transition from differentiation during pregnancy to successful milk secretion at lactation has been divided into two stages, termed secretory differentiation and secretory activation [144]. The first phase, secretory differentiation, begins at mid- 


\section{A}
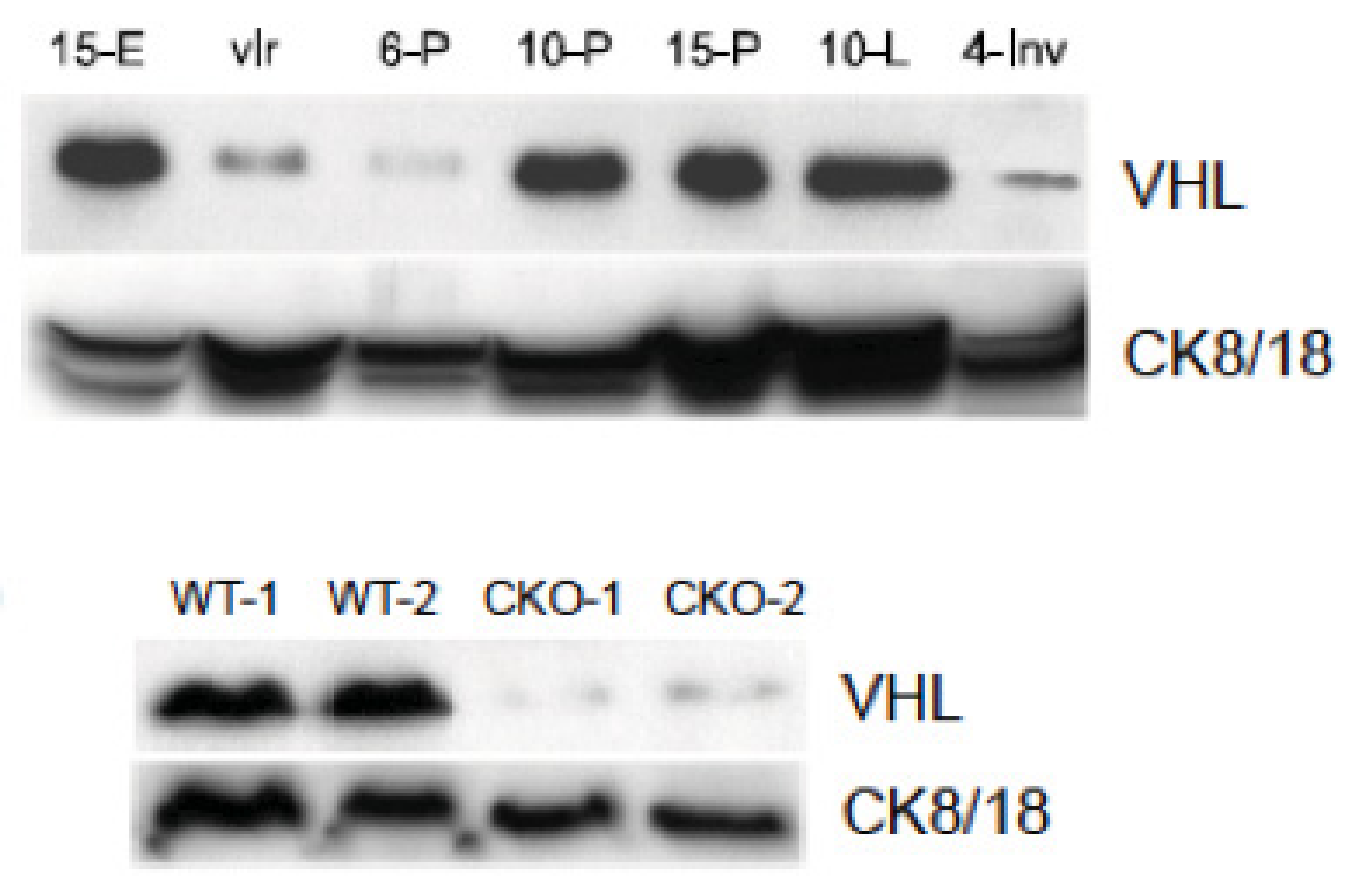

Figure 2-1. Expression of murine VHL increases over the course of normal mammary gland development as epithelial cell content increases.

(A) Western blotting for VHL was performed using tissue whole cell extracts (WCE, 100 $\mu \mathrm{g} / \mathrm{lane}$ ) prepared from mid-gestation embryos (15-E) or whole mammary glands harvested from mice over the course of gestation and lactation. Extracts were prepared from mature nulliparous (vir) mice, at day 6,10 or 15 of pregnancy (6-P, 10-P, 15-P), at mid-lactation (10-L) or 10-L followed by 4 days of forced involution (4-Inv). The same blot was stripped and reprobed with antibodies to cytokeratins 8/18 (CK8/18) to indicate the relative abundance of mammary epithelial cell (MEC) content at each stage of development. Note the increased expression of VHL from day 6 of pregnancy to day 10 of pregnancy that persists throughout lactation, and then dramatically decreases at 4-Inv, a peak period of MEC cell death. The virgin sample was overloaded as indicated by the increased expression of $\mathrm{CK} 8 / 18$ in this lane relative to sample prepared from 6-P mice.

(B) To confirm efficient deletion of Vhlh in response to Wap-Cre-mediated recombination (conditional knockout, $\mathrm{CKO}$ ), tissue WCE were prepared from whole glands isolated from two independent control (WT) or CKO lactating dams during the third round of lactation. VHL expression is reduced by $>90 \%$ when normalized for MEC content based on CK8/18 expression.

Data generated by T.N. Seagroves. 
gestation with the production of significant quantities of milk protein and lipid. The second phase, secretory activation, depends on the completion of secretory

differentiation, and is coordinated with the birth of pups to begin lactation. Therefore, we next analyzed tissue histology at day 15 of pregnancy (15-P), a period well within the secretory differentiation phase. At this stage, differentiation may be visualized by H\&E staining by the presence of large cytoplasmic lipid droplets (CLD) within MEC as well as the presence of proteinaceous material in the alveolar lumen, which is likely comprised of milk proteins that accumulate in preparation for lactation [145].

At day 15 of pregnancy, alveoli formed in $\mathrm{Vh}^{-/-\mathrm{MEC}}$ glands were not differentiated since the alveolar lumens remained closed (Figure 2-2B). This was in contrast to the control glands (Figure 2-2A), which contained alveoli with defined lumens and the presence of CLD. Moreover, there appeared to be fewer alveoli per field in $\mathrm{Vhlh}^{-/ \mathrm{MEC}}$ glands compared to controls. To determine if there were differences in MEC proliferation at this stage, the percentage of MEC incorporating bromodeoxyuridine (BrdU) was measured. The average percentage of cells positive for BrdU incorporation decreased by $30 \%$ in $\mathrm{Vhlh}^{-/ \mathrm{MEC}}$ glands compared to control glands $(5.2 \% \mathrm{vs} .7 .5 \%)$ (Figure 2-2E). To confirm these results, we also compared the MEC content of glands at day 15 of pregnancy by automated quantitation of images of whole-slide digitized H\&Estained sections using a pixel density algorithm as described in the methods. The number of pixels corresponding to the mammary epithelium $/ \mathrm{mm}^{2}$ of tissue was decreased by $22 \%$ ( $\mathrm{n}=4 \mathrm{mice} /$ genotype, Figure 2-2F). By day 18 of pregnancy (18-P), just prior to parturition, H\&E-stained slides showed that the glands containing $V h l h^{-/-\mathrm{MEC}}$ appeared to maintain a reduced number of alveoli per field (Figure 2-2D). Moreover, there were also lobuloalveolar units within the gland that did not appear to be well-differentiated.

Automated pixel density analysis revealed that epithelial content was reduced by $\sim 50 \%$ at 18-P ( $\mathrm{n}=3$ mice/genotype, Figure 2-2F).

To assay for molecular changes in differentiation, real-time PCR was also performed using cDNA prepared from 18-P glands (Figure 2-2G). Three markers of secretory differentiation were analyzed, adipose differentiation-related protein (ADRP; also known as adipophilin), ß-casein (CSN2) and whey acidic protein (WAP). Whereas $\beta$-casein is one of the earliest markers of differentiation induced by pregnancy hormones, Wap and Adrp are expressed during later stages of differentiation [146, 147]. Antibodies to ADRP have also been used to immunostain the surface of CLD produced by differentiated MEC [145]. In addition, we assayed for changes in expression of two direct HIF-1 target genes, Glut-1 and Vegf. Compared to control glands, expression of Adrp, Csn 2 and Wap mRNA decreased by 2.6-fold, 6.2-fold and 46.4-fold, respectively, in $V h l h^{-/-M E C}$ glands. As would be expected when $V h l h$ is deleted, expression of Glut 1 mRNA increased by $40 \%$ and the expression of $V e g f$ mRNA increased by 10.5 -fold. Together, these results demonstrate that loss of VHL activity delays both MEC proliferation and secretory differentiation during pregnancy and impacts gene expression. 
Figure 2-2. Reduced alveolar proliferation and differentiation in response to $V h l h$ deletion via MMTV-Cre.

Mammary glands were harvested from floxed control (A, C, H, J) or $\operatorname{Vhlh}^{-/ \mathrm{MEC}}(\mathrm{B}, \mathrm{D}, \mathrm{I}$, $\mathrm{K})$ glands at day 15 (A-B; 15-P, 200x magnification) or day 18 of pregnancy (C-D; 18-P, 200x magnification), day 10 of the first lactation (H-I; 10Lac\#1) or day 10 of the second lactation (J-K; 10Lac\#2), sectioned and stained with H\&E. All scale bars represent 10 $\mu \mathrm{m}$. (A-B) Note the decreased number of alveoli per field and the lack of differentiation in Vhlh $^{-/-\mathrm{MEC}}$ glands at 15-P. (C-D) Fewer alveoli were also observed in $\mathrm{Vhlh}^{-/ \mathrm{MEC}}$ glands at 18-P. (E) The percentage of BrdU+ cells per total number of MEC was determined at day 15-P in individual mice ( $\mathrm{n}=5 /$ genotype), and the means compared between genotypes. (F) The number of hematoxylin-positive pixels associated with $\mathrm{MEC} / \mathrm{mm}^{2}$ gland scan area was compared using whole-slide scanned, digitized slides by the automated Aperio Pixel Density algorithm as described in the methods. The mean number of pixels plus the SEM is presented. (G) Real-time PCR was performed to compare expression of Adrp, ß-casein (Csn2), Wap, Glut1 and Vegf mRNAs in control and $\mathrm{Vhlh}^{-/ \mathrm{MEC}}$ glands harvested at day 18 of pregnancy. The normalized fold-change (FC) in gene expression is expressed relative to expression levels observed in cDNA samples from control glands. Blue or orange bars indicate those genes for which relative expression decreased or increased, respectively. The average fold-change plus the SEM is presented. $(\mathrm{H}-\mathrm{K}) \mathrm{H} \& \mathrm{E}$-stained sections were prepared from mammary glands harvested from mid-lactation dams during the first (10Lac\#1; H-I, 400x magnification) or second (10Lac\#2; J-K, 200x magnification) cycles of lactation from the same cohort of test and control mice. As expected, the morphology of the wild type gland remains relatively unchanged from the first to the second lactation (H vs. J).

Data generated by D.L. Peacock, T.N. Seagroves and L.P. Schwab. 

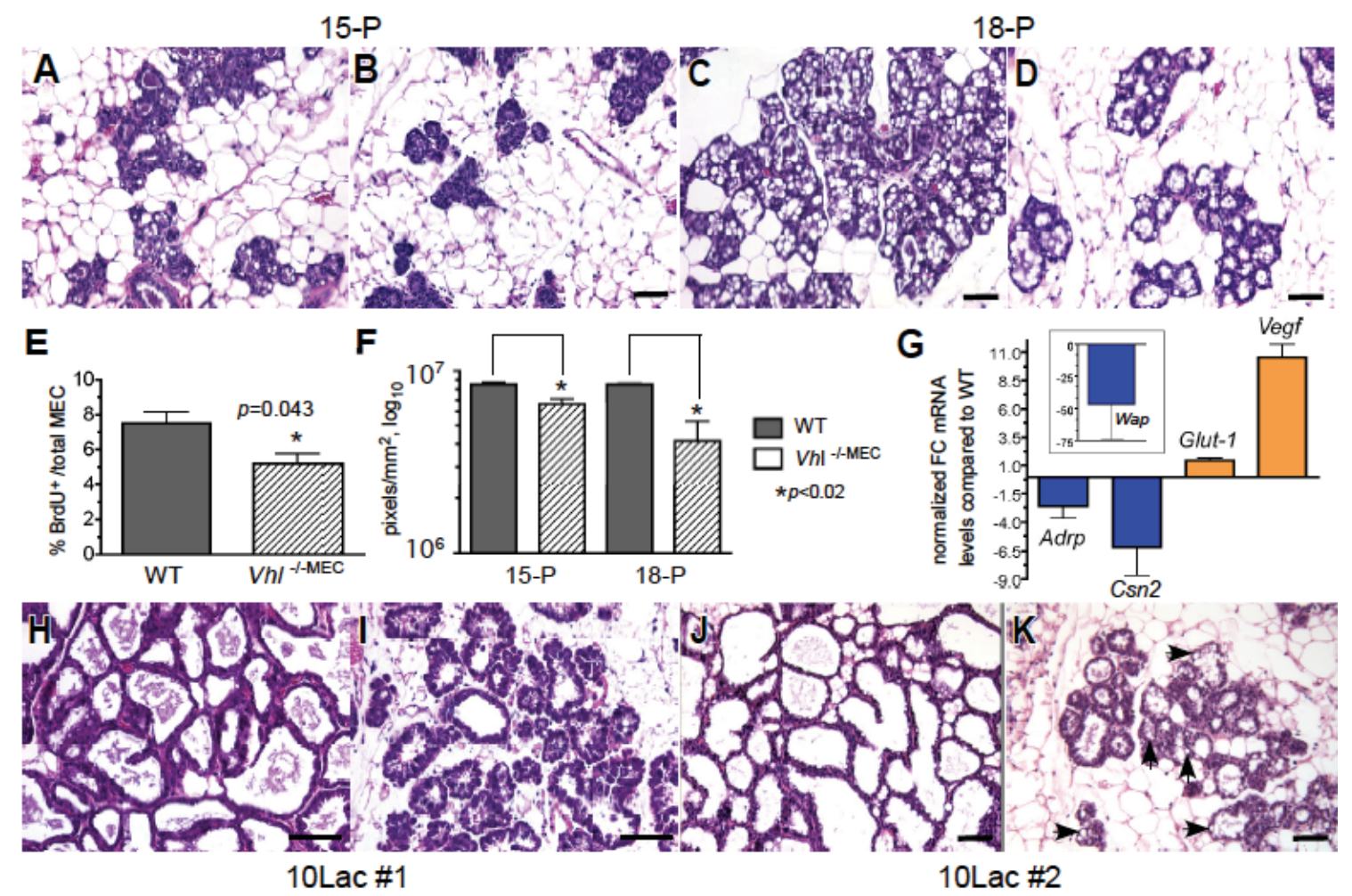


\section{Deletion of Vhlh Results in Subtle Changes in Secretory Activity during First Lactation}

In contrast to the strong lactation phenotype we observed previously upon deletion of Hifla in MEC during the first period of lactation [50], lactating mammary glands comprised of $\mathrm{Vhlh}^{-/ \mathrm{MEC}}$ were able to successfully produce milk, as no striking changes in pup size were apparent at weaning of the first litter. However, upon close histological examination of biopsied glands, subtle defects in MEC secretory function were observed in lactating glands at day 10 of lactation, a period of peak milk production (Figure 2-2H-I). After a weaning period of 2-3h to permit milk accumulation in the gland, much less evidence of milk production was present in the $V h l h^{-/ M E C}$ alveoli compared to controls. Moreover, the majority of MEC within lobuloalveolar units of $\mathrm{Vhlh}^{-/ \mathrm{MEC}}$ glands were not completely flattened with a basal nuclear location, as was observed in control glands. In addition, the $V h l h^{-/-\mathrm{MEC}}$ alveoli exhibited reduced lumen size and areas of adipose tissue were still visible in each field.

Multiple lobuloalveolar units present within the $V h l h^{-/-M E C}$ glands also exhibited an abundance of cytoplasm that stained heavily basophilic. This feature is indicative of hypersecretory, apocrine metabolism, and is reminiscent of the Arias-Stella reaction. The Arias-Stella phenomenon refers to a pathology of the endometrium that is found in a subset of pregnant women characterized by nuclear hyperchromatism, cytoplasmic swelling and vacuolization of apocrine epithelium due to a premature response to pregnancy hormones [148]. Therefore, at first lactation, deletion of Vhlh produces a mild secretory phenotype.

In contrast, during the second round of lactation, more severe defects were noted. Histological examination of biopsied glands revealed a more dramatic inhibition of alveolar differentiation and secretion at day 10 of lactation (Figure 2-2J-K). As expected, the histology of the glands of wild type mice between the first and second lactation periods was indistinguishable. Because the phenotypes observed in MMTV-Crepositive transgenic females did not manifest until pregnancy and lactation, we then proceeded to confirm these observations using the Wap-Cre transgenic line. Furthermore, by using Wap-Cre to delete $V h l h$, we avoided any potential indirect effects on mammary gland development due to the promiscuous expression of MMTV-Cre in other cell types besides the mammary epithelium [137].

\section{Deletion of Vhlh Contributes to Progressive Lactation Failure}

The primary goal of utilizing Wap-Cre transgenic mice to delete Vhlh in continuously bred, multiparous females was to determine if its deletion, and therefore, HIF- $1 \alpha$ constitutive expression, was sufficient to result in mammary tumorigenesis. We also sought to determine if use of Wap-Cre transgenic mice would phenocopy the Vhlh deletion lactation phenotype observed in the MMTV-Cre transgenic mice. To perform continuous breeding, littermate Vhlh control (wild type; WT) or $V h l h^{-/ M E C}$ females were constitutively housed with FVB males since prior studies have shown that continuous 
breeding dramatically accelerates mammary tumorigenesis $[149,150]$. Therefore, we reasoned that if loss of the tumor suppressor Vhlh is sufficient for mammary tumor initiation, then mammary tumors would be observed in aged, multiparous females due to selection for stochastic mutations in $V h l h^{-/-M E C}$. Furthermore, continual breeding would maintain constitutively high Cre transgene levels due to pregnancy hormone stimulation of the Wap promoter [147].

In order to observe changes in histology during successive rounds of lactation, individual thoracic or inguinal mammary glands were harvested from the same cohort of lactating dams during survival surgery at the first, third and fifth period of lactation (Lac\#1, Lac\#3 or Lac\#5) and sections prepared for H\&E analysis. As observed following deletion of Vhlh by the MMTV-Cre transgene, at day 10 of the first round of lactation, although there were mild defects in secretory activation in $V h l h^{-/-M E C}$ glands

(Figure 2-3A vs. Figure 2-3B), pups were able to thrive and appeared to be normal size at weaning of the first litters. In contrast, by the second round of lactation, there was a $27 \%$ decrease in the mean pup weight at day 10 of lactation (Figure 2-3E), although these litters survived and could be successfully weaned to independent cages at 21 days of age.

In contrast, by the third round of lactation, $100 \%$ of litters born to Wap-Cre positive dams were visibly severely runted throughout lactation and pup weight was decreased by $62 \%$ at day 10 of lactation (Figure 2-3E). As evident in H\&E-stained sections of glands harvested during the third lactation period, there were dramatic reductions in the both the number of $V h l h^{-/-M E C}$ alveoli and the ability of the alveoli formed $\mathrm{Vhlh}^{-/ \mathrm{MEC}}$ to produce milk. Moreover, the majority of alveoli present were poorly organized, and in several areas, no distinct alveolar lumen could be observed. Whereas the entire lactating mammary gland of wild type multiparous mice is compromised of fully differentiated alveoli, in the $V h l h^{-/ M E C}$ glands, the paucity of alveoli present was striking. In fact, the $\mathrm{Vhlh}^{-/ \mathrm{MEC}}$ glands were only $20-30 \%$ filled with alveoli by the third round of lactation (Figure 2-3C). Alveoli exhibiting hypersecretory, basophilic cytoplasmic secretions were also frequently observed. By H\&E staining, there also appeared to be increased leukocyte infiltration and microvessel density around the remaining islands of alveoli, which became even more pronounced in the $\mathrm{Vhlh}^{-/ \mathrm{MEC}}$ glands after subsequent rounds of lactation (for example, Figure 2-3G, fifth lactation). However, patches of well-differentiated lobuloalveolar units could also be detected in the thrice parous gland. The non-uniform appearance of the lobuloalveolar units in the $\mathrm{Vhlh}^{-/-}$ MEC glands likely results from the mosaic expression pattern of the Wap-Cre transgene during pregnancy and lactation (Figure 2-4).

To test whether these impaired alveologenesis and differentiation phenotypes were incremental in nature and progressively escalated upon additional rounds of breeding, we then harvested glands from the same cohort of mice between days 2-10 lactation during the fifth lactation. No defects were noted in multiparous control glands (data not shown), however the phenotype of the multiparous $\mathrm{Vhlh}^{-/ \mathrm{MEC}}$ gland was even more pronounced. Only $10-15 \%$ of the $\mathrm{Vhlh}^{-/ \mathrm{MEC}}$ glands were filled with alveoli (Figure 2-3F-G) and the alveoli exhibited little evidence of differentiation or milk 


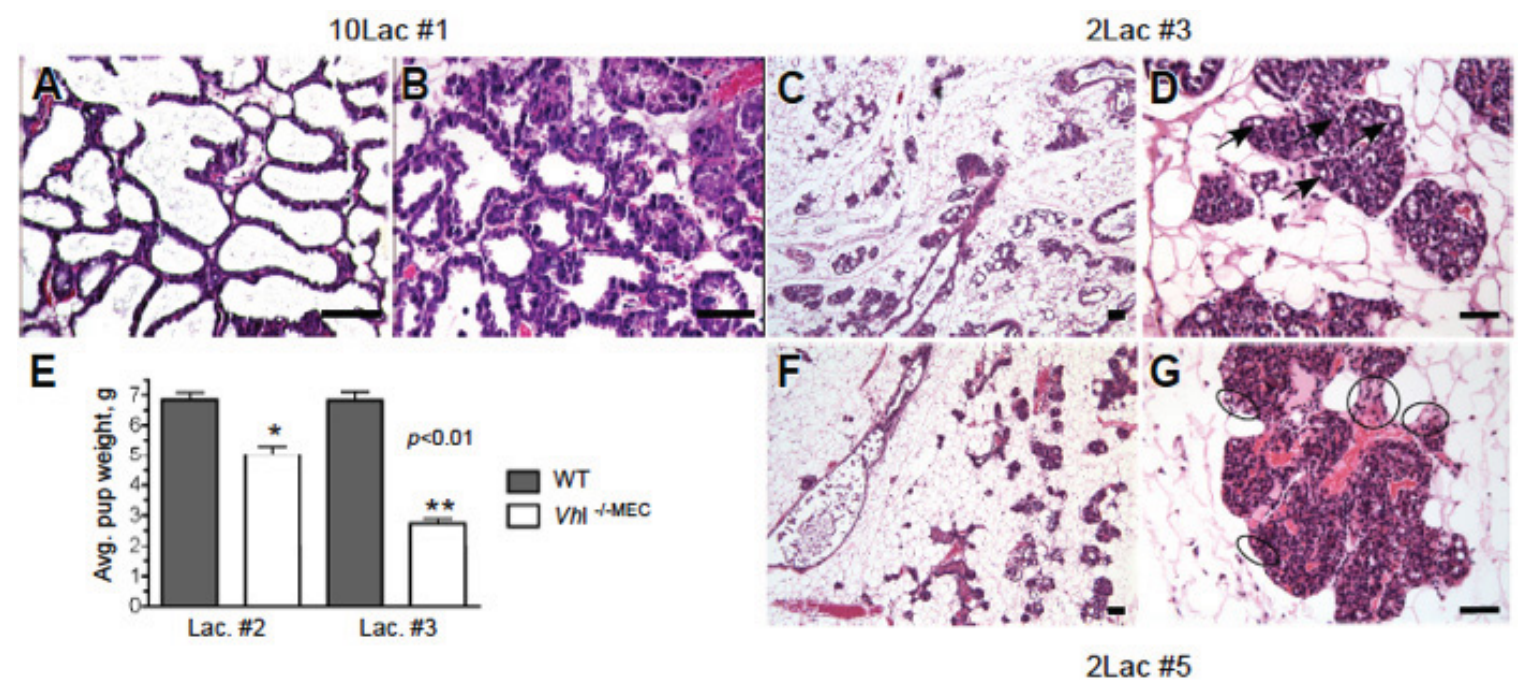

Figure 2-3. Deletion of Vhlh via Wap-Cre phenocopies results obtained with MMTV-Cre.

Mammary glands were harvested from the same cohort of control or Wap-Cre+ multiparous dams during successive rounds of lactation and stained with H\&E (A-D, FG). All scale bars represent $10 \mu \mathrm{m}$. (A-B) During the first cycle of lactation (10Lac\#1), the $V_{h l h}{ }^{-/ M E C}$ mammary gland accumulated less milk and there were subtle changes in MEC architecture, including the more frequent presence of basophilic cytoplasm in lobuloalveolar units (B) as compared to control glands (A), 400x magnification. Progressive changes in MEC content were determined by H\&E staining of glands biopsied from dams at day 2 of the third (C-D) or fifth (F-G) of lactation. As observed at low power magnification (50x), there are relatively few alveoli present in the $\mathrm{Vhlh}^{-/ \mathrm{MEC}}$ mammary gland during the third $(\mathrm{C}, 2 \mathrm{Lac} \# 3)$ or the fifth $(\mathrm{F}, 2 \mathrm{Lac} \# 5)$ rounds of lactation. As indicated from higher power images obtained from the same $V h l h^{-/ \mathrm{MEC}}$ mammary glands (D, G, 200x magnification), in response to Vhlh deletion there is a complete collapse of the alveolar lumen and there are multiple, large CLD still trapped in the MEC at the third lactation that should not be present during lactation (D, black arrows). By the fifth lactation, the lobuloalveolar structure is more disorganized, there are no distinct alveolar lumens and there appears to be increased microvessel density (D vs. G) and immune cell infiltration ( $\mathrm{G}$, black circles). The mean pup weight per litter $+/-$ SEM was calculated between control and $V h l h^{-/-M E C}$ dams ( $\mathrm{n}>5$ dams/genotype) on day 10 during the second (Lac. \#2) and third (Lac. \#3) cycles of lactation.

Data generated by T.N. Seagroves. 

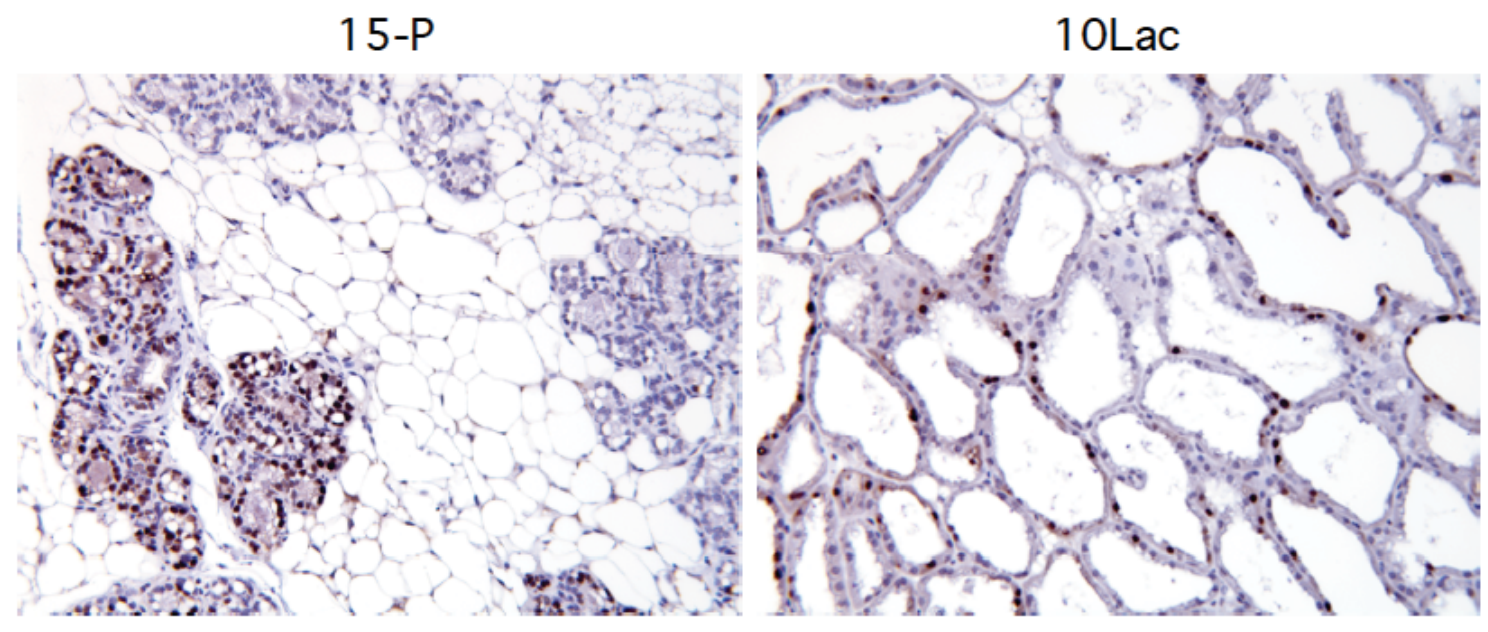

Figure 2-4. Mosaic nature of Cre expression under control of the Wap promoter. Cre (brown staining) was detected by immunostaining formalin-fixed tissue sections as described in [50]. The 15-P example demonstrates that Cre-positive lobules may be adjacently located to Cre-negative lobules. Cre expression continues to be mosaic in the lactating mammary gland.

Data generated by T.N. Seagroves. 
production. By the fifth round of lactation, no endogenous litters born to $V h l^{-/-\mathrm{MEC}}$ dams survived past the date of birth, therefore, pups obtained from lactating FVB females were cross fostered to Cre-positive dams daily to ensure a continuous suckling stimulus. These glands were harvested at day 2 of lactation since lactation failure was evident due to lack of milk in the stomachs of nursing pups. None of the $V h l h^{-/ M E C}$ glands harvested from dams bred five times contained areas that appeared by H\&E-staining to be differentiated enough to produce milk. In summary, extreme changes in gland structure were observed beginning at the third round of lactation, including loss of alveoli, decreased evidence of secretory differentiation, increased immune cell infiltration and increased microvessel density, which all became more prominent during subsequent rounds of breeding. Because the proportion of affected MEC increased with each period of gestation and lactation, it is possible that VHL is necessary for maintaining the regenerative potential of the secretory mammary epithelium.

\section{Deletion of Vhlh Does Not Promote Mammary Carcinoma in Aged Females}

Although we had expected to observe that deletion of a tumor suppressor that causes HIF-1 $\alpha$ over-expression would result in mammary tumorigenesis, we did not observe any hyperplastic alveolar nodules or carcinomas in any mammary glands biopsied from multiparous Wap-Cre-positive mice. No palpable masses or tumors were observed in any of the Vhlh conditional knockout females subjected up to eight pregnancies and maintained up to 24 months of age ( $n=9$ mice/genotype). Instead, as described, we observed a progressive loss of the secretory epithelium with each round of pregnancy. Therefore, it is clear that deletion of Vhlh in the MEC is not sufficient to promote mammary tumorigenesis. These data are consistent with the lack of report of mutations in $V H L$ in sporadic breast cancer.

Although no overt tumors were detected, we did observe in H\&E-stained sections from multiparous, lactating females $\left(3^{\text {rd }}-5^{\text {th }}\right.$ lactation period) features suggestive of mild dysplasia ( $n=5$ mice). These areas were estimated to occur in less than $3 \%$ of the epithelial cells. The dysplastic lesions were noted only during the lactation period in Crepositive dams that had been bred at least three times. For example, there were ducts oracini that contained multiple, fingerlike projections characterized by large MEC with dark, snout-like, amphophilic cytoplasms that projected into the ductal/alveolar lumens. The nuclei of these regions also were occasionally hyperchromatic. Within these regions, there were also pockets of cells that appeared to be syncitia with 2 or more nuclei observed per cell (Figure 2-5). However, these changes were not permanent since the no dysplastic regions were observed the same dams when contralateral glands were prepared for histology 14 days after removal of the litter. Therefore, we did not observe any direct evidence that loss of Vhlh transforms the mammary epithelium. 


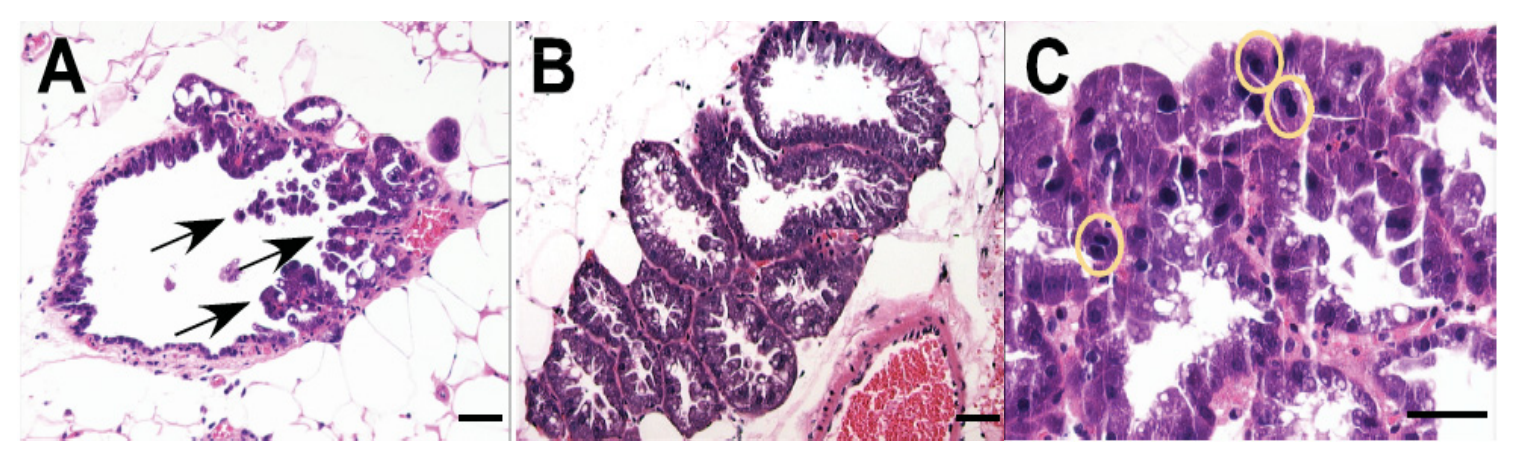

Figure 2-5. In multiparous dams, $V h l h^{-/-M E C}$ glands exhibit evidence of mild dysplasia.

Example of rarely observed fingerlike projections into the ducts, 200x magnification. (B) Example of the more commonly observed fingerlike projections into the alveolar lumen, 200x magnification. (C) Example of a region containing syncitia of bi-nucleated cells (yellow circles) as well as basophilic staining cells, 400x magnification. All scale bars represent $10 \mu \mathrm{m}$.

Data generated by T.N. Seagroves. 


\section{Constitutive Expression of HIF-1 $\alpha$, GLUT1 and VEGF, Increased Microvessel Density and Enhanced Recruitment of Leukocytes in Response to Vhlh Deletion}

To further characterize markers downstream of VHL in the $V h h^{-/-M E C}$ glands, including HIF-1 $\alpha$ and its direct transcriptional targets, Vegf and Glut1, we performed immunostaining on glands harvested from multiparous Wap-Cre transgenic females during the third lactation period. Of note, we had previously demonstrated that GLUT1 immunoreacitivity is strongly down-regulated in the normal mammary gland when Hifla is deleted in MECs [50]. Because high levels of VEGF can induce leukocyte infiltration and blockage of VEGF signaling can reduce immune cell infiltration into breast tumors [151], we also stained sections with a pan-leukocyte marker, CD45.

In wild type glands, HIF-1 $\alpha$ was detected primarily in the nucleus, as expected, and was expressed by approximately $60 \%$ of the acinar MEC (Figure 2-6A). In contrast, deletion of $V h l h$ caused up-regulation of HIF-1 $\alpha$ expression (Figure 2-6B). It should be noted that in response to Vhlh deletion, HIF-1 $\alpha$ immunoreactivity was observed in both the nucleus and the cytoplasm of MEC, but that no expression was detected in the more basally-located myoepithelial cells or in the cells immediately adjacent to the alveoli, which by H\&E-staining appeared to be infiltrating immune cells (Figure 2-6G, circled regions). The expression of GLUT1 (basolateral localization) and cytoplasmic VEGF was also strongly up-regulated in the $V h l h^{-/ M E C}$ glands compared to wild type glands (Figure 2-6C and 2-6D and Figure 2-6E and F), confirming that the HIF-1 $\alpha$ protein detected by immunostaining was transcriptionally functional.

\section{Epithelial-Specific Deletion of Vhlh Induces a Stromal Response}

Although deletion of $V h l h$ produces multiple MEC phenotypes, there were also severe defects in the mammary-associated stromal vasculature resulting from MECspecific deletion of $V h l$. This was first noticed during gross dissection of lactating glands. As shown in (Figure 2-6G and 2-6J), hlh $^{-/-M E C}$ glands harvested from thrice lactating multiparous dams were hypervascular, and the blood vessels leading to the mammary gland were dilated as compared to vessels in control glands. The $V h h^{-/-M E C}$ glands in multiparous mice also bled profusely during surgical resection, requiring heavy cauterization. Closer observation under a stereozoom microscope revealed the presence of large areas of hemorrhage within the glands (Figure 2-6J). In addition, there was very little accumulated milk within the $V h l h^{-\mathrm{MEC}}$ glands; the only milk present was restricted to few patches of presumably non-recombined alveoli. Finally, during milk collection, blood could occasionally be extracted along with milk from dams harboring $\mathrm{Vhlh}^{\text {-/MEC }}$, but not from wild type dams (data not shown). These data demonstrate VHL expression in the mammary epithelium is necessary for maintenance of the vasculature of the normal mammary gland and that VHL-regulated signals originating from the mammary epithelium control the proper branching and integrity of the mammary gland vasculature. To quantitate the changes in microvessel density between genotypes, Chalkley analysis was performed following immunostaining with anti-CD34 antibodies. As shown in 


\section{Figure 2-6. Changes in HIF-1 $\alpha$, GLUT1 and VEGF expression, microvessel density and immune cell infiltration in multiparous mice.}

(A-F) Deletion of Vhlh in the mammary epithelium results in uniform over-expression of HIF-1 $\alpha$ (B), GLUT1 (D) and VEGF (F) by MEC in thrice multiparous mice. Paraffin sections from formalin-fixed control (WT) or $\mathrm{Vhlh}^{-/ \mathrm{MEC}}$ mammary glands generated from Wap-Cre transgenic stocks were prepared from glands isolated from lactating dams during the third round of lactation. All scale bars represent $10 \mu \mathrm{m}$. Following antigen retrieval in citrate buffer, sections were stained with antibodies to HIF-1 $\alpha$, glucose transporter-1 (GLUT1), vascular endothelial cell growth factor (VEGF) or the panleukocyte marker CD45. (A-B) HIF-1 $\alpha$ signal (brown nuclear stain) was detected in approximately $60 \%$ of MEC in WT lactating tissue (A), whereas intense nuclear staining as well as cytoplasmic staining of HIF-1 $\alpha$ was observed in all MEC in which Vhlh was deleted (B). Note the lack of HIF-1 $\alpha$ staining in the stromal cells immediately adjacent to and surrounding the alveoli (B); the majority of these stromal cells have the morphology of myoepithelial cells (white arrowheads) or leukocytes (black arrowheads), 400x magnification. (C) As expected, GLUT1 is expressed by all WT MEC at lactation, but there was an extreme up-regulation of GLUT1 expression in the $V h l h^{-/-M E C}$ glands (D), 200x magnification. (E-F) Similarly, low levels of VEGF were expressed by WT glands (E), whereas expression of VEGF was strongly up-regulated in the $\operatorname{Vhlh}^{-/ \mathrm{MEC}}$ glands (F), 400x magnification. (G-H) Deletion of Vhlh results in hyper-vascularity and hemorrhage of the mammary gland. The gross appearance of thrice multiparous wild type $(\mathrm{G}-\mathrm{H})$ or $\mathrm{Vhlh}^{-/ \mathrm{MEC}}$ (I-J) thoracic mammary glands was compared by imaging live, anesthetized mice under a stereozoom dissecting microscope. The edge of the thoracic gland is shown in each low power field (G, I, 12.5x magnification). The dashed blue box indicates the area highlighted in panels $\mathrm{G}$ and I. In these panels individual alveoli and their supporting blood vessels can be visualized ( $\mathrm{H}, \mathrm{J}, 25 \mathrm{x}$ magnification). In control dams, all alveoli are fully distended with milk ( $\mathrm{G}$, white opaque material), and the uniform basketlike network of vasculature that surrounds each alveolus can be identified $(\mathrm{H})$. In contrast, in $\mathrm{Vhlh}^{-/-}$ MEC glands, the majority of vessels supporting the alveoli are hyper-dilated, overall vessel density appears to be increased and there are areas of hemorrhage ( $\mathrm{J}$, orange asterisk). The large intramammary vessels are also dilated (J, white arrows). Moreover, the paucity of milk-containing alveoli in the Vhlh null gland is striking, as there are only a few lobules capable of producing milk (yellow dashed oval). (K-L) Sections were stained with anti-CD45 antibodies. Very few CD45+ cells were detected in the stroma of wild type lactating glands ( $\mathrm{K}$, arrowheads), whereas there was an increase in the number of CD45+ cells present in the $V h h^{-/-M E C}$ glands (L). To quantitate changes in microvessel density and leukocyte infiltration, Chalkley analysis was performed following immunostaining for CD34 (M) and the mean percentage of CD45+ cells/total number of MEC was determined $(\mathrm{N})$. The mean Chalkley score and the percentage of CD45+/MEC \pm SEM is presented.

Data generated by D.L. Peacock, T.N. Seagroves and R.L. Krueger. 

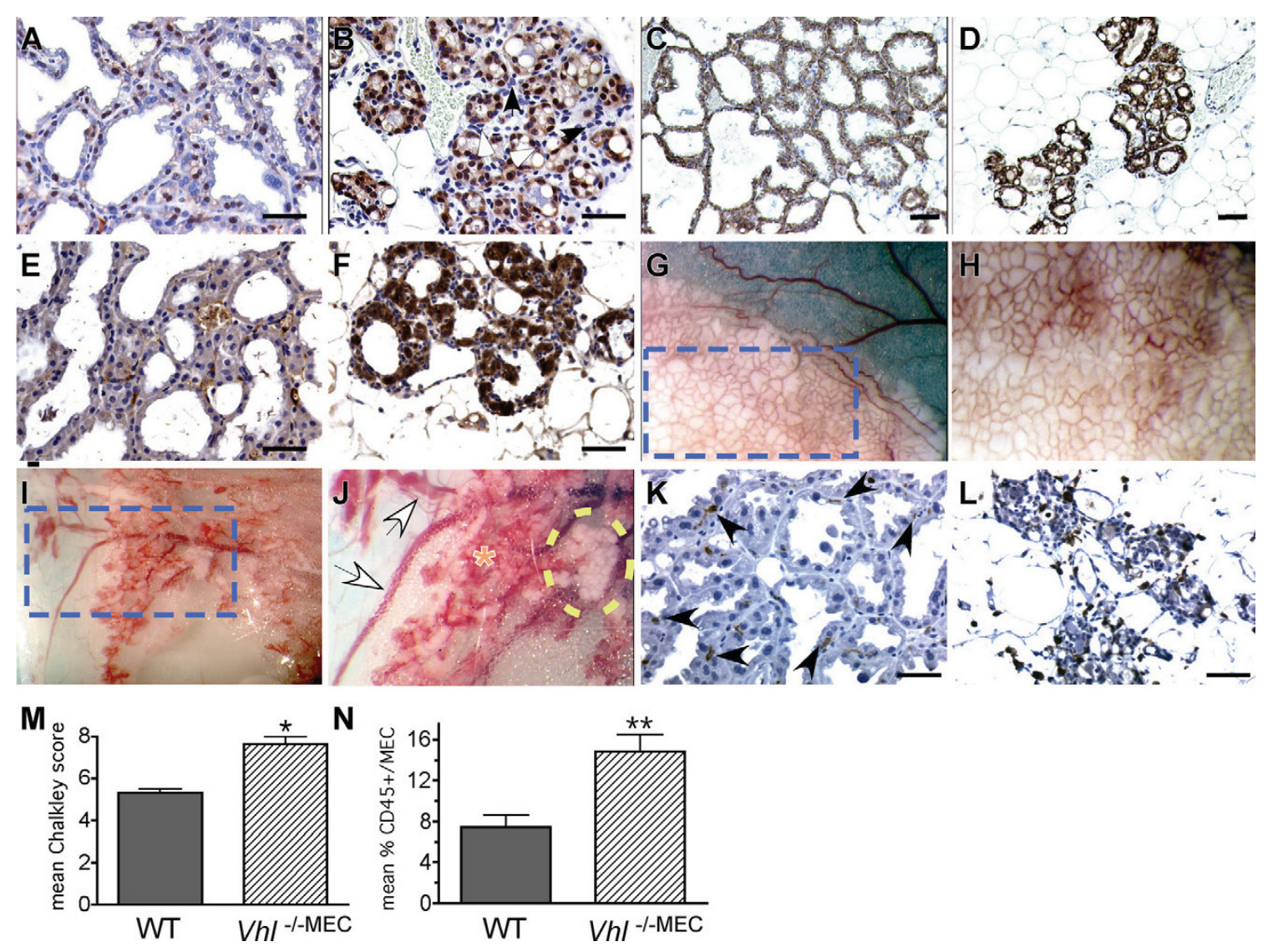
Figure 2-6M, there was a 30\% increase in the mean Chalkley score in multiparous Vhlh $^{-}$ /-MEC glands.

Finally, whereas CD45+ cells were occasionally detected in the stroma surrounding lobuloalveolar units in the wild type lactating gland (Figure 2-6K), we observed a dramatic increase in the number of CD45+ cells present in the stroma of the $\mathrm{Vh}^{-/ \mathrm{MEC}}$ glands immediately surrounding abnormal acini (Figure 2-6L), possibly due to increased production of VEGF. The mean percentage of CD45+ cells/total MEC increased $>2.0$-fold in multiparous $V h l h^{-/-M E C}$ glands (Figure 2-6N).

\section{Conditional Co-Deletion of Hif1a Does Not Rescue the VHL-Dependent Phenotypes}

Because HIF-1 $\alpha$ is a key substrate of VHL that at least partially confers the phenotypes observed in VHL disease, we then tested whether conditional co-deletion of Hifla could rescue the Vhlh-dependent phenotypes. To achieve deletion as early as possible during mammary gland development, the MMTV-Cre line D transgene was utilized. As shown in Figure 2-7, deletion of Hifla was not sufficient to rescue the phenotypes resulting from Vhlh deletion. In fact, during the first lactation period, there were fewer alveoli present in the double knockout gland (Figure 2-7C) than were observed in glands in which either Vhlh (Figure 2-3D) or Hifla (Figure 2-7B) had been singly deleted. Moreover, the few alveoli present in the co-deleted gland were more collapsed and poorly differentiated compared to alveoli present in either the control glands (Figure 2-7A) or the glands in which Hifla alone was deleted (Figure 2-7B). No pups survived that suckled the co-deleted gland, even during the first lactation period, therefore, exogenous pups were continuously cross-fostered to maintain the suckling stimulus.

To measure changes in gene expression, cDNA was prepared from glands biopsied from co-deleted or Vhlh-deleted glands on the date of birth, when secretory activation begins ( $\mathrm{n}=3$ mice/genotype). Relative to control glands, expression of Vegf mRNA was up-regulated 3.4-fold in Vhlh-deleted mice compared to 2.0-fold in codeleted glands. In addition, expression of $\beta$-casein (Csn2) mRNA was down-regulated $67 \%$ in Vhlh-deleted glands and 5.5-fold in co-deleted glands and the expression of Wap mRNA was decreased 50\% in Vhlh-deleted glands and 10.6-fold in co-deleted glands. Therefore, the decreased expression of differentiation markers in the co-deleted gland corresponded with changes observed by histology.

\section{Discussion}

We have generated the first conditional knockout mouse model to target deletion of $V h l$ and co-deletion of Vhlh and Hifla within the epithelium of the mammary gland. We have shown that in the normal murine mammary gland, VHL expression increases over the course of pregnancy as MEC content increases and then decreases sharply at involution when MEC regress. These data are supported by previous observations that 


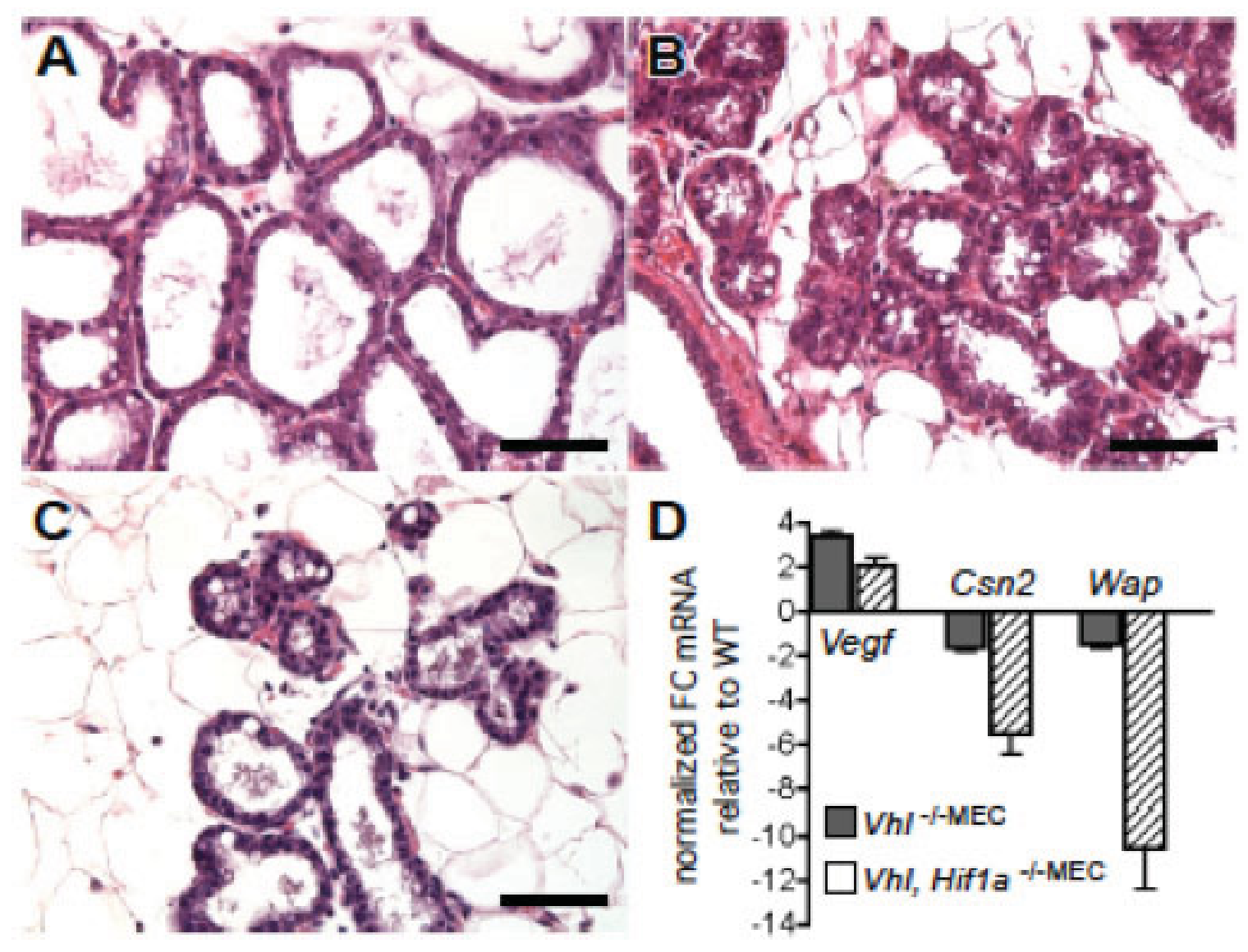

Figure 2-7. Co-deletion of Vhlh and Hifla via does not rescue the Vhl lactation phenotype.

Mammary glands were harvested from mice of each genotype at day 2 of lactation of the first gestation, sectioned and stained with H\&E. All scale bars represent $10 \mu \mathrm{m}$. In each case, deletion was achieved using the MMTV-Cre line D transgene. (A) Lactation is normal in Cre-negative Vhlh/Hifla floxed control dams. (B) Single deletion of Hifla via MMTV-Cre line D produces mammary glands with poorly differentiated alveoli and trapped CLD, as previously reported [50], although dams were capable of nursing litters. (C) Co-deleted Vhlh/Hifla ${ }^{-/ \mathrm{MEC}}$ glands contain relatively few alveoli, which are poorly differentiated and characterized by small lumens. (D) Expression of Vegf, ß-casein $(C s n 2)$ and Wap mRNA was compared between Vhlh $^{-/ \mathrm{MEC}}$ and Vhlh/Hifla ${ }^{-/ \mathrm{MEC}}$ glands on the date of birth, when secretory activation begins. The normalized fold-change (FC) in gene expression is expressed relative to expression levels observed in cDNA samples from control glands. The average fold-change (FC) +/- SEM is presented per genotype per gene (dark grey bars, single Vhlh deletion, hatched bars, Vhlh/Hifla co-deletion).

Data generated by D.L. Peacock, L.P. Schwab and T.N. Seagroves. 
expression of VHL is primarily localized to the epithelium rather than the stroma [141, $152,153]$. Conditional deletion of Vhlh in response to either MMTV-Cre or Wap-Cre transgene expression reduced alveolar proliferation and secretory differentiation during the first round of pregnancy.

Although there were subtle defects in MEC architecture during the first period of lactation, dams were able to complete lactation without impacting pup growth. In contrast, beginning during the second period of lactation, average pup weight was decreased. In addition, with each subsequent gestation, deletion of VHL resulted in the continued loss of alveoli capable of producing milk, leading to complete lactation failure. The potential of $V h l h^{-/ M E C}$ to re-populate the parous mammary gland with functional alveoli was strikingly reduced by the third lactation, which resulted in a decrease in average pup weight of $\sim 60 \%$. The progressive nature of this phenotype was revealed by the observations that both the number of alveoli $V h l h^{-/ M E C}$ and the differentiation potential of the MEC continued to decrease with each subsequent pregnancy until lactation completely failed by the fifth period of lactation. Changes in mammary epithelial cell (MEC) architecture and secretory function were also accompanied by changes in the mammary stroma, including increased microvessel density, hemorrhage and increased leukocyte infiltration.

Moreover, Vhlh deletion in the mammary epithelium and the downstream upregulation of HIF-1 $\alpha$ was not sufficient to induce mammary hyperplasias or carcinomas. These results are consistent with observations that no VHL mutations have been detected in sporadic breast cancer and the lack of reports of increased lifetime incidence of breast cancer in VHL patients [152]. Although mutations in $V H L$ may not play a role in breast cancer, recent studies have shown that $V H L$ mRNA levels are altered in breast cancer patients. For example, real-time PCR analysis of 124 breast cancer tissue samples indicated that levels of VHL mRNA are decreased in node-positive breast cancers, in patients with poor prognosis and in patients with metastases or high grade tumors [153]. These results imply that decreases in VHL expression may occur as breast cancer cells become less-differentiated and more aggressive, which would parallel with the observed timing of over-expression of HIF- $1 \alpha$ during tumor progression in breast cancer patients [84].

\section{A Role for VHL in Mammary Progenitor Cells}

The striking reduction in the number of alveoli observed in $V h l h^{-/ \mathrm{MEC}}$ glands with each sequential lactation, combined with the previously described ability of the Wap-Cre transgene to target a population of self-renewing, progenitor cells known as parityinduced MECs (PI-MECs) [142, 154], suggests that loss of Vhlh may inhibit the renewal capacity of the MEC. The PI-MECs are hormone-responsive progenitor cells that have the capacity to proliferate and to renew over several generations [142, 155]. It has been observed using the flox-STOP-ROSA26 reporter line of mice that the MEC exposed to Wap-Cre during the first gestation period constitute an increased percentage of the total regenerated mammary epithelium during subsequent pregnancy cycles, forming both 
ducts and alveoli comprised of luminal epithelial cells [142]. Therefore, the inability of Vhlh ${ }^{-/ \mathrm{MEC}}$ glands of multiparous mice to fully re-populate with alveoli and to achieve complete secretory activation upon multiple, successive pregnancies is particularly intriguing since the Wap-Cre transgene targets Cre expression to differentiated cells as well as the PI-MECs.

The most dramatic decreases in alveolar number accompanied by the reduced ability to differentiate fully to produce milk were noted in Wap-Cre transgenic females bred at least 3 times. Because the MEC derived from the PI-MEC population expressing Wap-Cre will expand with successive rounds of pregnancy and lactation, it is possible that there would be a larger proportion of Vhlh-deleted MEC present in the Wap-Cre+ glands with each successive round of lactation. This phenomenon could explain why the Vhlh deletion phenotypes were enhanced with each gestation period and why dams were able to successfully nurse their litters at the first and second lactations, but could not maintain their litters during subsequent lactations.

There is increasing evidence that the $V H L / H I F$ axis is involved in stem cell renewal, as reviewed by Keith et al. [156]. For example, in murine embryonic stem (ES) cells, HIF- $1 \alpha$ and HIF- $2 \alpha$ are expressed, but HIF- $2 \alpha$ is not transcriptionally active [157]. In human cancer cell lines, HIF-1 $\alpha$ has been shown to physically interact with the Notch intracellular domain and with $\beta$-catenin, which modulates transcriptional target gene activation $[158,159]$; both the Notch and Wnt/ß-catenin pathways genes are key players in stem cell regulation. The relationship of this axis to cancer stem cell biology is particularly intriguing since hypoxic regions of solid tumors are known to be highly resistant to radiation and chemotherapy [160] and since cancer stem cells are thought to represent the therapeutic-resistant fraction of tumors [161]. Therefore, anti-HIF therapies may enhance the clinical response to chemotherapy or radiation $[162,163]$.

In the normal murine mammary gland and in various mouse models of breast cancer, the pluripotent population of primitive stem cells defined by enriched selfrenewal in vitro and outgrowth potential in vivo has been defined as $\mathrm{CD}_{2} 4^{+}$(heat stable antigen) and CD29 ${ }^{\text {hi }}$ or CD49f ${ }^{\text {hi }}$ ( $\alpha 6$-integrin) [118, 121, 164-166]. A sub-population of the PI-MECs have been shown to co-express CD24/CD49f [167]. This population is thought to exhibit characteristics of a more committed progenitor MEC with limited pluripotent potential rather than a more primitive stem cell since most PI-MECs did not belong to the long-term label-retaining population [168]. Together, these observations are consistent with our data that that the predominant defect in $V h l h^{-/-M E C}$ glands from multiparous females is a failure of the alveoli to regenerate and to differentiate during pregnancy. We propose that the luminal progenitor cell population that has the potential to form ductal and alveolar luminal MEC decreases in response to loss of Vhlh.

Further investigation is warranted to determine if HIF-1 $\alpha$ also plays a direct role in regulation of mammary progenitor cell expansion or lineage commitment. Our hypothesis is not without precedent as HIF-1 $\alpha$ has been shown to act through the Notch3 pathway to promote breast cancer stem cell renewal when cells are cultured under hypoxic conditions [169] and since the Rich laboratory has found that the HIFs directly 
regulate cancer stem cell activity in glioblastomas [115]. In fact, in the glioma model, there was HIF-dependent, preferential expression VEGF and GLUT1 in the cancer stem cell population compared to the non-stem population [115].

\section{A Key Role for Local VEGF in Regulating the Mammary-Associated Vasculature}

The increased microvessel density, presence of hemorrhage and increased immune cell infiltration observed in multiparous glands may be due to the up-regulation of $V e g f$ mRNA levels, which was initially observed in $V h l h^{-/-\mathrm{MEC}}$ glands during the first round of pregnancy and lactation. By the third period of lactation, we also observed via immunostaining extremely high levels of VEGF protein localized to the mammary epithelium. These phenotypes contrast with our previous observations of the effects of conditional Hifla deletion in the mammary gland, in which we observed profound defects in alveolar differentiation without any impact on alveolar cell number/proliferation, changes in microvessel density or changes in $V e g f$ mRNA expression [50].

However, it is unlikely that VEGF acts alone to cause the Vhlh-dependent phenotype. This conclusion is derived from our observations that co-deletion of Hifla in the Vhlh null background only slightly reduced $V e g f$ mRNA levels, but enhanced the block in lactation when compared to deletion of either Hifla or Vhlh alone. It is known that VEGF and VEGF receptor (VEGFR) expression increases during pregnancy and lactation and that VEGF immunostaining switches from the stroma to the epithelium during late gestation and lactation $[170,171]$. Therefore, local VEGF produced by MEC is critical for lactation as demonstrated by conditional deletion of $V e g f$ in the mammary gland via expression of the cytokeratin-5 (K5)-Cre transgene. In these experiments, deletion of Vegf in MEC and myoepithelium resulted in decreased angiogenesis during pregnancy and blocked milk production at lactation [172]. Therefore, it would be of interest to determine if co-deletion of $V e g f$ could reverse the multiple vascular phenotypes observed in the $V h l h^{-/-M E C}$ mammary gland. In this scenario, Vegf would not be expected to rescue MEC secretory defects or the production of milk.

\section{Hif1a Deletion Cannot Rescue the Vhlh Phenotype, a Role for Other VHL- Regulated Targets}

Based on the strong correlation between loss of VHL function and constitutive HIF activity in VHL patients [173], we predicted that co-deletion of Hifla would at least partially rescue the Vhlh phenotype. We focused on deletion of Hifla in these studies rather than Hif $2 a$ since up to $80 \%$ of genes in breast epithelial cells are transcriptionally regulated by HIF- $1 \alpha$ rather than HIF- $2 \alpha[22,174]$. Moreover, in a panel of breast cancer cell lines, HIF-2 $\alpha$ expression was low to undetectable, also pointing to HIF-1 $\alpha$ as the primary regulator of the hypoxic response in the breast [175]. The dependence of breast cancer cells upon HIF-1 $\alpha$ for mediating the hypoxic response was later confirmed using 
specific siRNAs to either HIF-1 $\alpha$ or HIF- $2 \alpha$ and then analyzing expression of key HIF target genes [22].

Instead of ameliorating the Vhlh-dependent phenotypes, co-deletion of Hifla resulted in a further reduction in the number of alveoli at lactation. In addition, the few alveoli that formed exhibited even less evidence of secretory activity by histology than was observed for single deletion of either Hifla or Vhlh. In addition, dams harboring codeleted mammary glands were incapable of producing enough milk to support their litters even at the first round of lactation. These results suggest that in the context of the Vhlhdeleted gland, HIF-1 $\alpha$ over-expression acts in part to maintain pathways required for proper secretory differentiation and milk production. However, the phenotypes arising from deletion of Vhlh cannot solely reflect enhanced HIF-1 $\alpha$ activity as co-deletion of Hifla could not rescue lactation and instead amplified the impaired lactation phenotype. Therefore, additional VHL-regulated substrates or interacting proteins besides the HIFs must contribute to the pathology observed in the $V h l h^{-/ \mathrm{MEC}}$ glands.

There is increasing evidence that VHL regulates multiple proteins through HIFindependent pathways $[176,177]$, including via protein-interaction activities that are independent of its ability to target proteins for proteasome-dependent degradation. For example, VHL has been implicated in regulation of the physical integrity of cells and the extracellular matrix network, through modulation of microtubule stability [178] and the processing of the fibronectin extracellular matrix $[179,180]$. VHL also physically interacts with several other proteins [177]. For example, VHL interactions stabilize the putative tumor suppressor Jade-1 (gene for apoptosis and differentiation in epithelia), which then promotes ubiquitinylation of ß-catenin [181]. Finally, it is possible that deletion of $V h l h$ in the mammary epithelium may also lead to premature senescence independent of HIF $\alpha$ function via up-regulation of the cyclin-dependent kinase inhibitor p27, as recently described in mouse embryonic fibroblasts [182].

\section{A Role for VHL in the Prolactin Response}

The mammary gland is resilient and often compensates for lack of gene function in subsequent pregnancies by ameliorating the phenotype observed at first lactation. Our data suggest that VHL function is critical to regulate either the ability of progenitor cells to develop into differentiated epithelium. An alternate explanation is that the $\mathrm{Vhlh}^{\text {-/MEC }}$ are unable to respond to pregnancy hormones. Interestingly, the incremental loss of alveolar differentiation phenotype observed upon deletion of $V h l h$ is similar to that observed previously upon Wap-Cre mediated deletion of Jak2 in the murine gland, the kinase that activates STAT5 in response to prolactin. Conditional deletion of Jak2 completely blocks alveolar proliferation and differentiation, which is compounded by a failure to maintain differentiated cells during lactation [183]. Similarly, deletion of Stat5 via Wap-Cre, in which Stat5 is deleted after alveolar differentiation has initiated, results in a loss of differentiated cells, suggesting that STAT5 is also needed for maintaining alveolar differentiation [184]. Previous studies have shown that hypoxia can activate STAT5 through JAK2 in normal murine HC11 MEC and in MCF7 breast cancer cells 
[185], however, it is unclear whether STAT5 activation would also occur in response to deletion of Vhlh since HIF-1 $\alpha$ is constitutively expressed.

\section{Summary}

In conclusion, although VHL does not function as a classic tumor suppressor in the breast, we have shown for the first time that it is required to regulate proper development and expansion of the mammary epithelium during repetitive cycles of pregnancy and lactation. Since the mammary gland is not essential for survival of the individual animal, the conditional deletion model system we have developed will be useful to continue to dissect the general role of VHL in regulation of proliferation, differentiation and/or self-renewal of secretory epithelium. 


\section{CHAPTER 3. HYPOXIA-INDUCIBLE FACTOR $1 \alpha$ PROMOTES PRIMARY TUMOR GROWTH AND TUMOR-INITIATING CELL ACTIVITY IN BREAST CANCER}

\section{Introduction}

Over-expression of the oxygen-responsive transcription factor Hypoxia Inducible Factor (HIF)-1 alpha correlates with poor prognosis in breast cancer patients. The MMTV-PyMT (polyoma virus middle T) mouse is a widely utilized pre-clinical mouse model that resembles luminal breast cancer and is highly metastatic. Prior studies in the PyMT model demonstrated that HIF-1 is essential to promote carcinoma onset and lung metastasis, although no differences in primary tumor endpoint size were observed. Using a refined model system, we investigated whether HIF-1 is directly implicated in regulation of tumor-initiating cells (TICs) in breast cancer. Mammary tumor epithelial cells (MTECs) were created from MMTV-PyMT mice harboring conditional alleles of Hifla, followed by transduction ex vivo with either Adenovirus-beta-galactosidase or Cre to generate wild type (WT) and HIF-1 null (KO) cells, respectively. The impact of HIF-1 deletion on tumor-initiating potential was investigated using tumorsphere assays, limiting dilution transplantation and gene expression analysis. Efficient deletion of HIF-1 $\alpha$ reduced primary tumor growth and suppressed lung metastases, prolonging survival. Loss of HIF-1 led to reduced expression of markers of the basal lineage (K5/K14) in cells and tumors and of multiple genes involved in the epithelial to mesenchymal transition. HIF$1 \alpha$ also enhanced tumorsphere formation at normoxia and hypoxia. Decreased expression of several genes in the Notch pathway, Vegf and Prominin-1 (CD133) were observed in response to HIF deletion. Immunohistochemistry confirmed that CD133 expression was reduced in $\mathrm{KO}$ cells and in tumorspheres. Tumorsphere formation was enhanced in CD133 ${ }^{\text {hi }}$ versus CD133 ${ }^{\text {neg }}$ cells sorted from PyMT tumors. Limiting dilution transplantation of WT and KO tumor cells into immunocompetent recipients revealed $>30$-fold enrichment of TICs in WT cells. These results demonstrate that HIF-1alpha plays a key role in promoting primary mammary tumor growth and metastasis, in part through regulation of TICs. HIF-1 regulates expression of several members of the Notch pathway, CD133 and markers of the basal lineage in mammary tumors. Our results suggest that CD133, which has not been profiled extensively in breast cancer, may be a useful marker of TICs in the PyMT model. These data reveal for the first time that HIF-1 directly regulates breast TIC activity in vivo.

\footnotetext{
* Adapted with permission. Schwab, L. P., Peacock, D. P., et al. (2012). "Hypoxiainducible factor 1alpha promotes primary tumor growth and tumor-initiating cell activity in breast cancer." Breast Cancer Res 14(1): R6.
} 


\section{Methods}

\section{Animals}

Mice harboring two alleles of exon 2 of Hifla flanked by loxP sites (doublefloxed, DF) were provided by Dr Randall Johnson (University of California San Diego) on a mixed genetic background (129Sv-C57BL/6) and were described in full detail in [41]. Hifla stock mice were first backcrossed to the FVB/Nj strain (The Jackson Laboratory, Bar Harbor, ME, USA) for 11 generations prior to being bred to MMTVPyMT transgenic mice obtained from Dr Kent Hunter (National Cancer Institute, Frederick, MD, USA), which had previously been backcrossed to the $\mathrm{FVB} / \mathrm{Nj}$ strain. Lung metastasis induced by the PyMT transgene is highly penetrant in the $\mathrm{FVB} / \mathrm{Nj}$ background [186]. All procedures were approved by Institutional Animal Care and Use Committee at the University of Tennessee Health Science Center.

\section{Establishing Hif1a Wild-Type and Knockout Mammary Tumor Epithelial Cells}

Several mammary tumors $\left(>500 \mathrm{~mm}^{3}\right)$ were isolated from Hifla DF, PyMT+ bigenic female mice. Tumors were chopped with scalpels and then with razor blades, and the paste was digested with $1 \mathrm{mg} / \mathrm{ml}$ collagenase type III (Worthington Biochemical Corp, Lakewood, NJ, USA) in RPMI media containing 5\% fetal bovine serum (FBS) (5 $\mathrm{ml} / \mathrm{g}$ tissue) for 2 hours at $37^{\circ} \mathrm{C}$. Organoids were pelleted at $1,100 \mathrm{rpm}$, washed four times with digestion buffer and then plated into standard tissue culture plates in plating medium as described previously [187]. After 48 to 72 hours, the plating medium was switched to complete mammary epithelial cell growth medium (GIBCO DMEM: Nutrient Mixture F-12 (DMEM/F-12; Invitrogen, Carlsbad, CA, USA), 5\% FBS, $5 \mu \mathrm{g} / \mathrm{ml}$ insulin (Sigma-Aldrich, St Louis, MO, USA), $10 \mathrm{ng} / \mathrm{ml}$ recombinant murine epidermal growth factor (EGF, Invitrogen). At passage 6, MTECs were transduced with either adenovirus $\beta$-galactosidase (adeno- $\beta$-gal) or adeno-Cre at a multiplicity of infection (moi) of 80 plaque-forming units (pfu)/cell to generate WT and KO MTECs, respectively. Adenoviral transduction was repeated, and the deletion efficiency between WT and KO MTECs was confirmed by both quantitative RT-PCR (qRT-PCR) and Western blot analysis. After adenoviral transduction, MTECs were weaned to medium containing only $2 \%$ FBS (DMEM/F-12 + 2\% FBS).

For subcultivation, cells were rinsed twice with Puck's A saline, then incubated for up to 60 minutes at $37^{\circ} \mathrm{C}$ in a $3: 1$ solution of dispase II $/ 0.25 \%$ trypsin reconstituted in Puck's A. No ethylenediaminetetraacetic acid (EDTA) was utilized to subcultivate cells because treating cells with trypsin-EDTA changed tumor cell morphology from an epithelial (cuboidal) to a mesenchymal-like (spindle) appearance. All cells were passaged less than 30 times before use in tumorsphere or in vivo assays. Spent media were routinely tested for mycoplasma using the MycoAlert Kit (Lonza, Basel, Switzerland). 
All cells were grown either at normoxia in an air-jacketed $\mathrm{CO}_{2}$ incubator $(5 \%$ $\mathrm{CO}_{2}$; SANYO, Wood Dale, IL, USA) or at hypoxia $\left(0.5 \% \mathrm{O}_{2}, 5 \% \mathrm{CO}_{2}\right)$ in a multigas incubator (SANYO) in which $\mathrm{N}_{2}$ gas displaces $\mathrm{O}_{2}$. Cells were exposed acutely ( $\leq 6$ hours) or chronically ( $>6$ hours to several days) to hypoxia and were removed from chronic hypoxic exposure only for brief periods to change the media.

For immunostaining of cultured cells, WT or KO cells passaged with a dispasetrypsin mixture were plated into tissue culture-treated chamber well slides (BD Biosciences, Franklin Lakes, NJ, USA or EMD Millipore Corporation, Billerica, MA, USA), grown to $60 \%$ to $80 \%$ confluence and then post fixed for 20 minutes at room temperature with $2-4 \%$ paraformaldehyde (PFA)-PBS, followed by permeabilization with $0.5 \%$ Triton X-100 for 5 minutes. Cells stained with CD133 were not permeabilized. Primary antibodies to CD133, Troma-I (K8), cytokeratin 14 (K14) and cytokeratin 5 (K5) were incubated overnight at $4^{\circ} \mathrm{C}$. All cells were stained with 4',6-diamidino-2phenylindole (DAPI) prior to being mounted with VECTASHIELD Mounting Medium (Vector Laboratories, Burlingame, CA, USA).

\section{Western Blot Analysis}

Insoluble material remaining after preparation of whole-cell extract (WCE) was reextracted in high-salt $(\mathrm{HS})$ solution $(400 \mathrm{mM} \mathrm{NaCl})$ buffer as described in Chapter 2, except that the deubiquitinase inhibitor N-ethylmaleimide (NEM) was added to a final concentration of $0.5 \mu \mathrm{M}$. HS-WCE was resolved on $3 \%$ to $8 \%$ Tris-acetate gels ( 1 to 10 $\mu \mathrm{g} / \mathrm{lane}$; Invitrogen) and transferred onto polyvinylidene fluoride membranes prior to blocking with $5 \%$ milk and enhanced chemiluminescence-based detection of antibody complexes.

\section{Gene Expression}

Total RNA was prepared using RNA-Bee RNA isolation reagent (amsbio, Lake Forest, CA, USA), and RNA quality was confirmed by the Agilent 2100 Bioanalyzer (Agilent Technologies, Santa Clara, CA, USA) assay. RNA with an RNA integrity number $>9.0$ was used to prepare cDNA using the High-Capacity cDNA Reverse Transcription Kit (Applied Biosystems, Foster City, CA, USA). qRT-PCR was performed using optimized primer and 6-carboxyfluorescein-labeled probe sets designed using Universal ProbeLibrary Assay Design Center software (Roche Applied Science, Indianapolis, IN, USA) as described previously in Chapter 2. To control for cDNA input (40 to $80 \mathrm{ng} /$ reaction) when using cultured cells as the source of RNA, the crossing point (Cp) values were normalized based on the expression of the integrator complex subunit 3 (Ints3) gene, which is expressed at moderate levels in the mammary gland. This gene changes $<20 \%$ between WT and KO cells cultured at normoxia or hypoxia and in WT and KO tumors by Illumina whole-genome expression arrays (Illumina Inc, San Diego, CA, USA) (TNS, personal observations). To compensate for any changes in epithelial 
content in whole tumors between genotypes, because only the tumor epithelium is deleted for Hifla, Ints3-normalized Cp values were also normalized to Krt18 (cytokeratin 18).

\section{Cell Growth and Invasion Assays}

MTECs were grown in normoxic culture (ambient air; $5 \% \mathrm{CO}_{2}$ ) or hypoxic culture $\left(0.5 \% \mathrm{O}_{2}, 5 \% \mathrm{CO} 2\right)$ in medium buffered with $25 \mathrm{mM}$ 4-(2-hydroxyethyl)-1piperazineethanesulfonic acid (HEPES). On the day before enumeration, either 350,000 cells/well in 6-well format (complete growth medium) or 100,000 cells/well in 12-well format ( $2 \%$ FBS medium) were seeded into multiwell plates. In all cases, the medium was changed postplating, but was not replenished for the duration of the experiment. Cells were harvested after culture for $0,24,48,72$ or 96 hours of culture at normoxia and hypoxia. All cells were plated in triplicate or quadruplicate per genotype/oxygen tension/time point. Each replicate was counted by hemacytometer, and counts were verified using an Accuri personal flow cytometer after gating against cell debris (BD Biosciences).

For invasion assays, WT and KO MTECs that had been gradually weaned to medium supplemented with $0.5 \%$ FBS were cultured overnight in serum-free DMEM/F12 medium. The next day 25,000 cells were plated onto control inserts or Matrigel-coated transwell inserts (BD Biosciences) and attracted to wells containing complete growth medium with 5\% FBS. Cells were plated in triplicate per genotype/oxygen tension. The mean cell invasion index corrected for random migration was calculated after 48 hours according to the manufacturer's instructions. Changes in invasion are expressed as a fold changes relative to the invasion index observed for WT cells cultured at normoxia (fold change $=1.0)$.

\section{Mammary Tumor Epithelial Cell Transplant into FVB Recipients}

MTECs dissociated into single cells with $0.05 \%$ trypsin-EDTA were counted using a hemacytometer and diluted into HBSS. When transplanted into recipients at relatively low density ( $\leq 500$ cells/gland), cells were diluted 1:1 (vol:vol) with growthfactor reduced Matrigel-Hank's balanced salt solution (HBSS). At higher densities, cells were resuspended in HBSS alone. Cells were kept on ice until injection into the right inguinal mammary fat pads $(10 \mu \mathrm{l})$ of 3-week old female $\mathrm{FVB} / \mathrm{Nj}$ recipients (The Jackson Laboratory) using a 26-gauge PT2 needle mounted on a Hamilton syringe, followed by clearing of the endogenous epithelium. Recipients were palpated one or two times per week, and outgrowths were measured with digital calipers to calculate tumor volume as described previously [126]. 


\section{Tissue Histology and Immunostaining}

Tumors were harvested from anesthetized mice and flash-frozen in liquid nitrogen for preparation of RNA or protein, or they were fixed in $10 \%$ neutral buffered formalin (NBF) for 6 hours at room temperature for histological staining $(\mathrm{H} \& \mathrm{E})$ and immunostaining. Paraffin-embedded sections ( 5 to $7 \mu \mathrm{m})$ were immunostained after antigen retrieval $(1 \times$ citrate buffer), followed by development with a VECTASTAIN Elite ABC Kit and ImPACT DAB (diaminobenzidine) substrate (Vector Laboratories). Alternatively, to prepare frozen sections suitable for CD133, Troma I, K14 and K5 immunofluorescent staining, anesthetized mice were perfused intracardially with $10 \%$ NBF. Tumor tissue was postfixed for 10 minutes in NBF at room temperature prior to cryoprotection overnight at $4^{\circ} \mathrm{C}$ in $30 \%$ sucrose-PBS, embedding in OCT medium and preparation of $10 \mu \mathrm{m}$ sections by cryostat. Sections used for visualizing the keratins were postfixed for an additional 10 minutes in $10 \% \mathrm{NBF}$ prior to $0.5 \%$ Triton $\mathrm{X}-100$ permeabilization.

\section{Lung Metastasis and Survival Studies}

Lungs were either harvested from recipients at the same time as primary tumors (at a volume of approximately $1,000 \mathrm{~mm}^{3}$ ), 8 weeks after primary tumor resection (500 to $750 \mathrm{~mm}^{3}$ ) or when recipients subjected to tumor resection were moribund as indicated by decreased body condition scores and panting. Lungs were inflated with $10 \% \mathrm{NBF}$ and postfixed in NBF overnight. Paraffin-embedded sections $(7 \mu \mathrm{m})$ representing every 100 $\mu \mathrm{m}$ of lung tissue were obtained for each paraffin block, and all sections were stained with $\mathrm{H} \& \mathrm{E}$. The individual $\mathrm{H} \& \mathrm{E}$-stained section containing the highest number of metastases per recipient, as evaluated by counting each slide under a light microscope at $\times 50$ original magnification, was used to determine the mean of metastases per genotype.

\section{Tumorsphere Culture and Immunostaining}

WT or KO MTECs were briefly trypsinized, washed and strained (40 $\mu \mathrm{m}$ filter) to obtain single cells. The presence and viability of single cells were verified by viewing trypan blue-stained cells using a hemacytometer, and only cells with $>90 \%$ viability were used in sphere assays. Single cells were resuspended in serum-free DMEM-F-12 mammosphere media containing $20 \mathrm{ng} / \mathrm{ml}$ mouse recombinant EGF, $20 \mathrm{ng} / \mathrm{ml}$ basic fibroblast growth factor, $1 \times$ B27 (all from Invitrogen) and $4 \mu \mathrm{g} / \mathrm{ml}$ heparin (SigmaAldrich, St Louis, MO, USA) as described previously [188]. Primary tumorspheres were derived by plating 30,000 single cells/well into six-well ultra-low-adhesion dishes. Secondary and tertiary tumorspheres were plated at 5,000 to 10,000 cells/well and 2,000 cells/well, respectively. Dishes were cultivated at normoxia or hypoxia $\left(0.5 \% \mathrm{O}_{2} ; 5 \%\right.$ $\mathrm{CO}_{2}$ ) for 10 to 14 days prior to enumeration of spheres. Individual spheres $\geq 100 \mu \mathrm{m}$ from each replicate well ( $n \geq 12$ wells/genotype/oxygen tension) were counted under a dissecting microscope. The percentage of cells capable of forming spheres, termed the "sphere formation efficiency" (SFE), was calculated as follows: [(number of spheres 
formed/number of single cells plated) $\times 100$ ]. End point tumorspheres were collected from ultra-low-adhesion dishes, washed with PBS, then flash-frozen for preparation of RNA or dried onto slides for 10 to 15 minutes at $37^{\circ} \mathrm{C}$. Slides were postfixed with $4 \%$ PFA-PBS for 15 minutes and immunostained. All slides were stained with DAPI prior to being mounted in either ProLong Gold antifade reagent (Invitrogen) or VECTASHIELD Mounting Medium (Vector Laboratories).

\section{Tumor Digestion and Flow Sorting}

All mammary gland tumors used for flow-sorting experiments were harvested from intact Hifla DF, MMTV-PyMT+ (equivalent to HIF-1 $\alpha$ WT) transgenic female mice at a size of $>300 \mathrm{~mm}^{3}$ but $<1,500 \mathrm{~mm}^{3}$. Necrotic areas were removed from solid tumor tissue, and solid tissue was cut into small fragments with scissors. Tumor tissue was then weighed and chopped for 5 minutes with scalpels, followed by 5 minutes of chopping to a fine paste with razor blades. The tissue paste was digested in digestion buffer $($ DMEM/F-12, $1 \times$ gentamicin, $1 \times$ antibiotic-antimycotic (Sigma-Aldrich), 300 $\mathrm{U} / \mathrm{mL}$ collagenase type III (Worthington Biochemical Corp) and $100 \mathrm{U} / \mathrm{mL}$ hyaluronidase (Sigma-Aldrich)) for 1 to 2 hours at $37^{\circ} \mathrm{C}$ at $125 \mathrm{rpm}(10 \mathrm{~mL} / \mathrm{g}$ tissue). Digested tissue was pelleted by centrifugation, and the red blood cells were lysed with a solution of $0.8 \% \mathrm{NH}_{4} \mathrm{Cl}$-HBSS. To obtain single cells, organoids were further digested with $0.25 \%$ trypsin-EDTA for up to 10 minutes at $37^{\circ} \mathrm{C}$ and washed with serumcontaining medium to inactivate trypsin, followed by a final digestion for 10 minutes at $37^{\circ} \mathrm{C}$ in $5 \mathrm{mg} / \mathrm{ml}$ dispase II (Roche Applied Science)/Puck's A solution containing 1 $\mathrm{mg} / \mathrm{mL}$ DNase I (Roche Applied Science). The cell suspension was passed through a 40 $\mu \mathrm{m}$ filter insert, and this flow-through was then passed through a flow cytometer tube with a $35 \mu \mathrm{m}$ filter cap insert to further enrich for single cells. Viability post-digestion was routinely assessed by trypan blue staining. Only cells with viability $>85 \%$ were subjected to antibody staining.

Isolated single cells were resuspended to a density of up to 5 million cells $/ \mathrm{ml}$ in flow buffer (HBSS, 2\% FBS, $10 \mathrm{mM}$ HEPES) and incubated for 1 hour on ice with the biotin- or fluorophore-conjugated antibodies. These antibodies included a biotinconjugated anti-mouse hematopoietic lineage panel supplemented with anti-mouse CD31-biotin, anti-mouse CD133-phycoerythrin (CD-133-PE) and anti-mouse CD24fluorescein isothiocyanate (CD24-FITC), each at a 1:100 dilution. Secondary antibody incubation (streptavidin-allophycocyanin) in flow buffer was performed for 30 minutes on ice. After being stained, cells were strained through a clean flow cytometer tube with a $35 \mu \mathrm{m}$ filter cap. To determine cell viability, either SYTOX Blue ( $1 \mu \mathrm{M}$; Invitrogen) or 7AAD ( $1 \mu \mathrm{g} / \mathrm{ml}$; BD Biosciences) was added to each flow tube sample 10 to 20 minutes before flow cytometry analysis.

Cells were subjected to cytometric profiling using a $100 \mu \mathrm{m}$ nozzle at a sheath pressure of 20 psi on a BD Biosciences FACSAria flow cytometer (maintained by the University of Tennessee Health Science Center Flow Cytometry and Sorting Core) equipped with violet $(404 \mathrm{~nm})$, blue $(488 \mathrm{~nm})$, green $(532 \mathrm{~nm})$ and red $(635 \mathrm{~nm})$ lasers and applying FACSDiva software. All sample and collection tubes were maintained on 
ice. BD Biosciences CompBeads stained with either CD133-PE or CD24-FITC were used to set the compensation gates, and cells stained with isotype-matched secondary antibodies were used as controls for staining specificity. Cells heat-killed for 30 minutes at $65^{\circ} \mathrm{C}$ were used to gate against dead cells positive for SYTOX Blue or 7-AAD. Lineage-negative $\left(\mathrm{Lin}^{\mathrm{neg}}\right)$ cells were identified by gating against cells positive for the mouse lineage panel (BD \# 559971) plus CD31-biotin. Because almost 100\% of cells isolated from late-stage carcinomas of the PyMT-transgenic mouse were positive for CD24-FITC as previously described [118], viable, Lin $^{\text {neg }}$ mammary tumor cells were gated in a two-way sort for CD133 ${ }^{\text {hi }}$ versus CD133 ${ }^{\text {neg }}$ cells.

Sorted cell samples were collected directly on ice into $15 \mathrm{~mL}$ conical tubes precoated with $20 \%$ FBS, and a subset of collected cells was subjected to postsorting analysis to verify the purity and viability of the sorted populations. Sorted cell populations were immediately pelleted, washed to remove FBS and counted using a hemacytometer prior to reconstitution in mammosphere medium and plated in six-well ultra-low-adhesion dishes (Corning Life Sciences, Corning, NY, USA) at the indicated densities. FACSDiva plots were imported into FlowJo version 7.0 software (Tree Star Inc, Ashland, OR, USA) for data analysis and creation of histogram plots.

\section{Limiting Dilution Transplantation}

WT or KO MTECs were briefly trypsinized, washed with HBSS and serially diluted into 1:1 HBSS/Matrigel. Six dilutions per genotype $(500,200,100,50,25$ or 10 cells $/ 10 \mu \mathrm{l}$ ) were introduced into the right inguinal cleared fat pads of $\mathrm{FVB} / \mathrm{Nj}$ recipients. Tumor-initiating potential was defined as the ability to form a palpable tumor mass $>5$ mm diameter (approximately pea-sized). The estimated TIC frequency was calculated using Extreme Limiting Dilution Analysis (ELDA) software [189]. Fisher's exact $\left(\chi^{2}\right)$ test was used as a complementary approach to compare TIC activity between WT and KO cells at each cell density evaluated (GraphPad Prism version 4.0 software; GraphPad Software, Inc, San Diego, CA, USA). All animals were palpated twice weekly until surgical resection of the primary tumor or euthanasia.

\section{Image Acquisition}

Western blots were scanned at 600 dpi and imported into Photoshop software (Adobe Systems, Inc, San Jose, CA, USA). Digital images of immunostained or H \& Estained tissue sections and cells cultured on chamber well slides were captured using a Leica DM6000 upright fluorescence microscope (Leica Microsystems, Inc, Buffalo Grove, IL, USA) mounted with a SPOT Insight cooled charge-coupled device camera (SPOT Imaging Solutions, Inc, Sterling Heights, MI, USA) and imported into Photoshop using SimplePCI software (Hamamatsu Photonics, Sewickley, PA, USA). All images between genotypes and conditions per experiment were digitally captured for the same exposure time. Immunostaining images were not digitally altered to reduce background or to adjust brightness or gain. Confocal images of spheres were captured every $0.75 \mu \mathrm{m}$ with a Zeiss LSM210 microscope system (Carl Zeiss, Thornwood, NY, USA). 
Appropriate gain and black level settings were determined based on spheres incubated with secondary antibody alone. All images were captured using the same gain and exposure settings, and upper and lower thresholds were set using the range indicator function. No additional background correction algorithms were applied. Digital slices were merged into a single, composite image using Zeiss Zen software.

\section{Results}

\section{A Mammary Tumor Model with Constitutively Deleted HIF-1 $\alpha$}

We observed that HIF-1 $\alpha$ expression generally increased during tumor progression in MMTV-PyMT mice as previously observed in patients [84], thereby confirming that the MMTV-PyMT model is appropriate for these studies

(Figure 3-1). To generate a parental pool of MTECs, mammary tumors were isolated from Hifla floxed (DF), PyMT + females (FVB/Nj background), and epithelial cells were transduced with ether adeno- $\beta$-gal or adeno-Cre to create HIF- $1 \alpha$ WT and KO cell lines, respectively. Efficient Hifla deletion was confirmed by qRT-PCR and Western blot analysis (Figure 3-1).

During expansion, MTECs were weaned from complete growth medium to medium supplemented only with $2 \%$ FBS, as low-serum medium was previously utilized to evaluate cell growth [126]. The levels of HIF-1 $\alpha$ detected by Western blot analysis following acute ( 0 to 6 hours) or prolonged ( $>6$ hours) hypoxia was determined using WT MTECs. On the initial day of hypoxia exposure, cells were $80 \%$ confluent prior to transfer to a hypoxic chamber for 24, 12, 8, 6 and 3 hours prior to harvest. Cells taken from normoxic culture (labeled as time $t=0$ ) were harvested at the same time as cells that had been exposed to hypoxic conditions for 24 hours. Similar to the profile observed in Hep3B cells [190], HIF-1 $\alpha$ peaked by 3 hours of exposure to $0.5 \% \mathrm{O}_{2}$, and, by 24 hours, expression had attenuated to basal levels (Figure 3-2A). This decrease in HIF-1 $\alpha$ expression may be due HIF-1 $\alpha$ 's role in transcriptional regulation of the prolyl hydroxylase (PHD) enzymes, which mediate HIF-1 $\alpha$ 's turnover by the proteasome, thereby creating a negative feedback loop [191].

In contrast, when WT cells were cultured in the presence of serum and EGF, HIF$1 \alpha$ levels were not attenuated by prolonged hypoxia exposure (Figure 3-3), and, in fact, HIF-1 $\alpha$ stabilization at hypoxia remained robust for at least 24 hours. Compared to the levels of HIF-1 $\alpha$ expression observed in cells cultured in 2\% FBS alone, EGF treatment increased HIF-1 $\alpha$ levels approximately four-fold at normoxia (time $t=0$ ), and this upregulation increased to $>10$ - or $>20$-fold following 3 or 24 hours in hypoxic culture, respectively. The effect was not due to the differences in serum or insulin concentrations between culture media because WT cells cultured in 5\% FBS $+5 \mu \mathrm{g} / \mathrm{ml}$ insulin without EGF expressed levels of HIF-1 $\alpha$ similar to those of cells cultured in $2 \%$ FBS alone (data not shown). Overall, these results indicate that EGF is a potent regulator of HIF-1 $\alpha$ expression in normoxic PyMT mammary tumor cells, as previously observed in SK-BR-3 


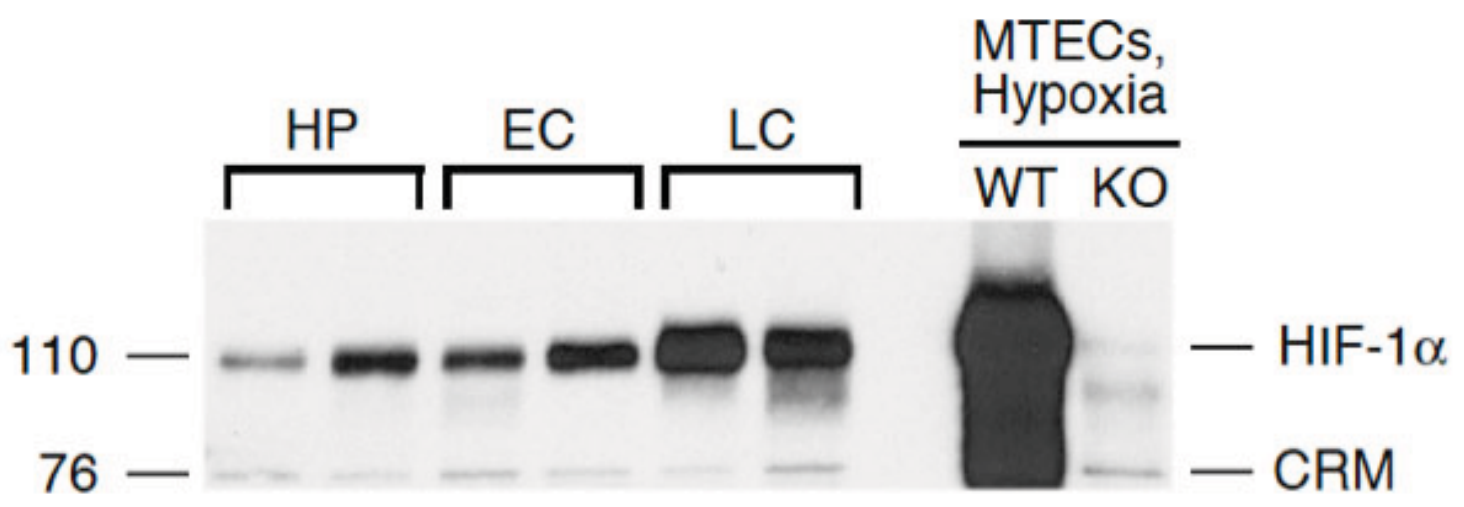

Figure 3-1. HIF-1 $\alpha$ expression increases during tumor progression in the MMTV-PyMT model and confirmation of HIF-1 $\alpha$ deletion.

HS-WCE protein extracts were prepared from two individual transgenic MMTV-PyMT female mice (FVB/ $\mathrm{Nj}$ strain) between 6-9 weeks of age. By palpation, each mouse had at least one mammary gland with hyperplastic (HP; palpable as grainy), early carcinoma $\left(\mathrm{EC},<250 \mathrm{~mm}^{3}\right)$ or late carcinoma $\left(\mathrm{LC},>500 \mathrm{~mm}^{3}\right)$ lesions, which was expected, since tumor progression among glands is asynchronous in this model. Two independent glands (one from each mouse) per stage were utilized to prepare HS-WCE for western blotting. As shown, HIF-1 $\alpha$ expression generally increases during progression, and is most abundant in late stage carcinomas. Included as controls are HS-WCE prepared from WT and KO MTECs grown to $80 \%$ confluence followed by hypoxic exposure for $6 \mathrm{~h}$ at $0.5 \%$ $\mathrm{O}_{2}$. A cross-reactive band (CRM) at $\sim 76 \mathrm{kDa}$ was detectable when using lot $\mathrm{E} 2$ of the Novus Biologicals anti-mouse HIF-1 $\alpha$ primary antibody (NB 100-479). KO cells were transduced twice with Adenovirus-Cre in monolayer culture at 80-100 p.f.u./cell in order to achieve $>99 \%$ deletion efficiency as determined by qRT-PCR and western blotting. 
Figure 3-2. Effect of HIF-1 $\alpha$ deletion upon growth and invasion.

A. WT MTECs grown to $80 \%$ confluence were subjected hypoxia culture for the indicated number of hours for up to $24 \mathrm{~h}$, or cells were continued to be cultured at normoxia, such that the $\mathrm{t}=0$ sample was harvested on the same day as the $\mathrm{t}=24 \mathrm{~h}$ hypoxic sample. HS-WCE were resolved on 3-8\% Tris-Acetate gels and blotted to PVDF membrane, which was divided horizontally at approximately $60 \mathrm{kDa}$. The top half of the blot was blotted for HIF-1 $\alpha$ and the lower portion was blotted for lamin (loading control) to avoid the need to strip and reprobe the blot. B. Growth curve of WT and KO MTECs cultured at normoxia (Nor) or hypoxia (Hyp) in growth medium supplemented with 5\% FBS + EGF (left panel) or with 2\% FBS (right panel). For cells grown in 5\% FBS + EGF, a representative graph is shown, in which the mean cell number \pm S.E.M. per time point of quadruplicate wells per genotype/oxygen tension is plotted per time point. For cells grown in $2 \% \mathrm{FBS}$, the grand mean of cell number \pm S.E.M. is presented, calculated as an average of the mean cell number observed per replicates per time point, as observed in three replicate experiments. All data was analyzed by two-way ANOVA $\left(*^{*}<0.05\right)$. C. The mean fold-change in invasion was normalized to the invasion index observed for WT cells cultured at normoxia $(\mathrm{FC}=1.0)$. Data represent the mean fold change in invasion observed in three independent experiments. All columns were compared to each other using one-way ANOVA analysis with a Bonferroni post-test, ${ }^{*} p<0.05$. 
A

$\begin{array}{lllllll}\text { Time (hours) } & 0 & 3 & 6 & 8 & 12 & 24\end{array}$

$$
\begin{aligned}
& 110-\square=-\mathrm{HIF}-1 \alpha \\
& 65-\longrightarrow-\operatorname{lamin} \mathrm{A}
\end{aligned}
$$

B

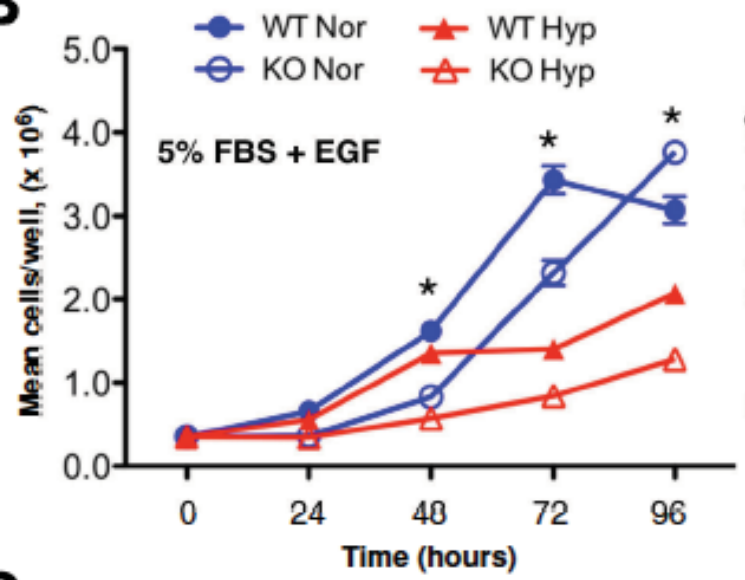

C
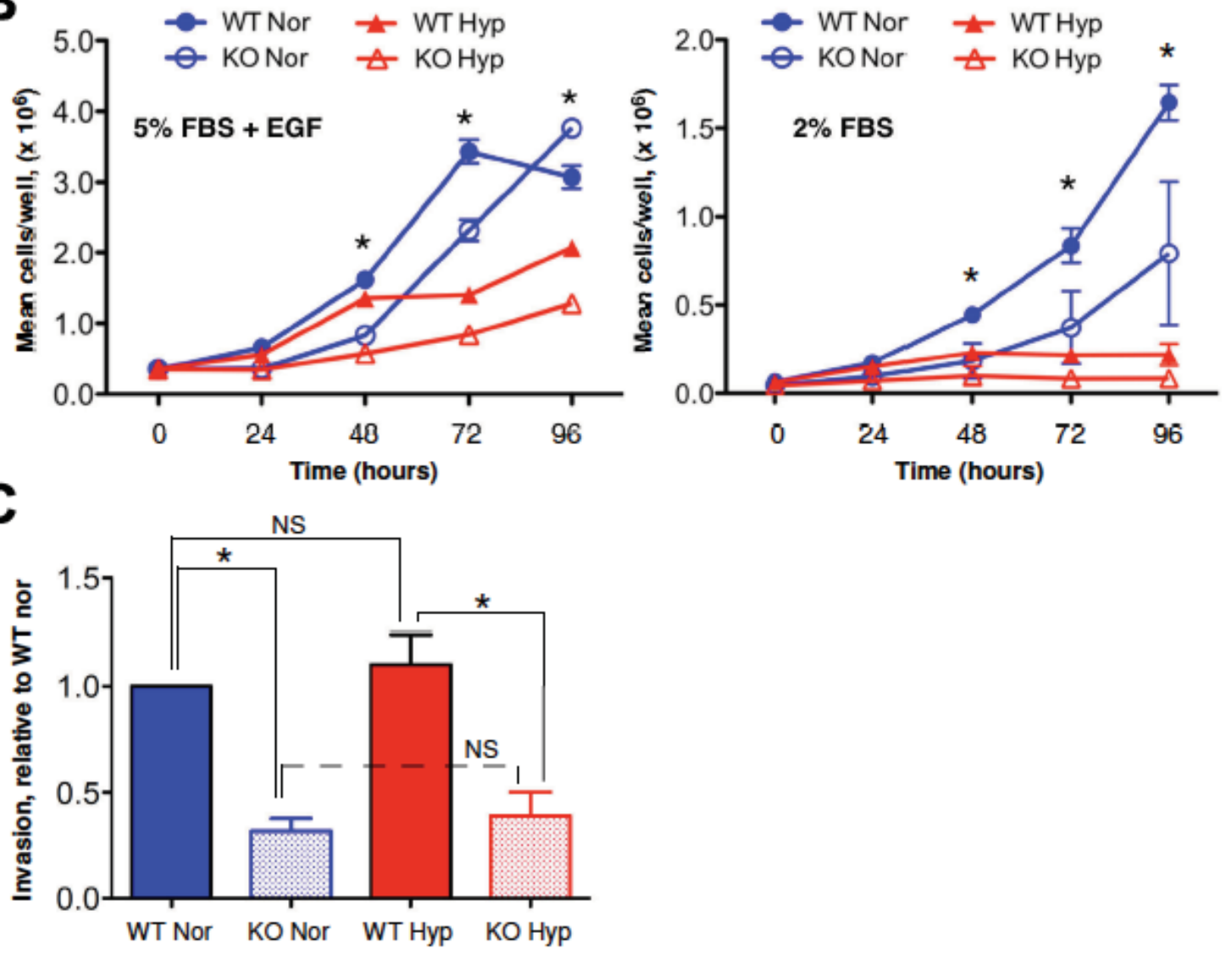


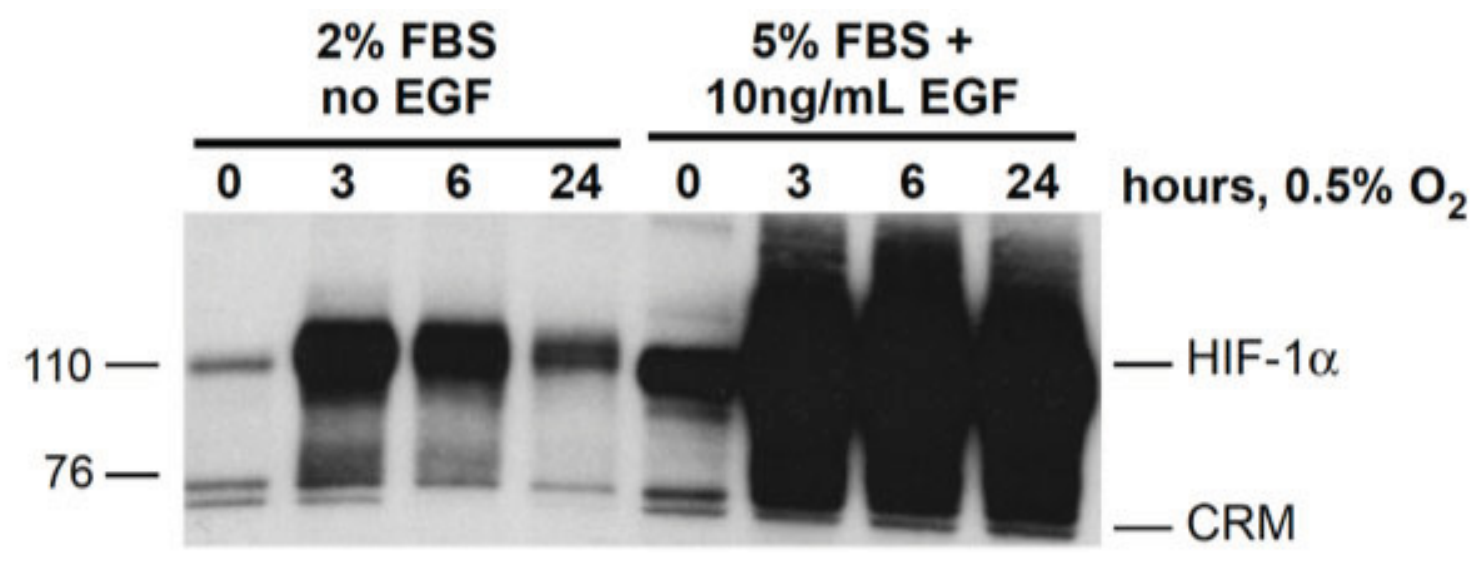

Figure 3-3. HIF-1 $\alpha$ protein expression increases in response to EGF treatment at normoxia, and EGF prolongs HIF-1 $\alpha$ stabilization at hypoxia.

WT MTECs were either cultured in complete growth medium (containing 5\% FBS +5 $\mu \mathrm{g} / \mathrm{mL}$ insulin $+10 \mathrm{ng} / \mathrm{mL}$ EGF) or in medium supplemented with $2 \%$ FBS only for at least five passages prior to re-plating to test the effect of EGF treatment on HIF-1 $\alpha$ expression. Cells grown to $80 \%$ confluence were incubated at hypoxia for the number of hours indicated $(3,6,24)$. All cells were put into hypoxic culture beginning $24 \mathrm{~h}$ prior to harvest of the $\mathrm{t}=0 \mathrm{~h}$ time point, such that cells at the $\mathrm{t}=0 \mathrm{~h}$ time point (normoxic control) were harvested at the same time as the $\mathrm{t}=24 \mathrm{~h}$ hypoxia time point. Cells were exposed to hypoxia for the indicated number of hours prior to removal from culture and the immediate extraction of HS-WCE. HIF-1 $\alpha$ was detected using lot E2 of NB 100-479. 
and MCF-7 cells [192], and that EGF prolongs HIF-1 $\alpha$ stabilization under hypoxic conditions.

\section{HIF-1 $\alpha$ Promotes Cell Growth and Invasion}

To determine whether the HIF-1 $\alpha$ KO PyMT+ MTECs derived from ex vivo adenoviral transduction behave similarly in vitro as previously described [126]; cell growth was compared in WT and KO cells cultured at normoxia and hypoxia. Cell growth was also compared for WT and KO cells cultured in complete or minimal $(2 \%$ FBS only) growth medium. By 48 hours of culture, and then throughout the time course, there was a statistically significant difference between WT and KO cell number at normoxia and hypoxia for each medium formulation (Figure 3-2B); overall, fewer KO than WT cells were observed following exposure to either oxygen tension. Cells cultured in complete growth medium grew faster than those in reduced serum. In cells grown in $5 \%$ FBS + EGF, more KO than WT cells were often observed by 96 hours of culture at normoxia (Figure 3-2B), owing to the acidic environment of the superconfluent WT cells as reported previously [193]. As expected, hypoxia exposure decreased the rate of growth for both WT and KO cells, although the difference between WT and KO cell density was the most striking following extended hypoxia exposure ( $\geq 72$ hours).

Cells cultured in 2\% FBS medium were gradually weaned to reduced serum $(0.5 \% \mathrm{FBS})$ prior to use in invasion assays since immediate serum withdrawal resulted in massive cell death even when cells had been routinely cultured in medium containing $2 \%$ FBS. Deletion of Hifla reduced invasion at normoxia by 3.9 -fold and by 3.5 -fold at hypoxia (Figure 3-2C). In contrast, significant induction of invasion by WT cells during hypoxia was not observed as previously described [126]. Although there was a trend toward increased invasion, the differences were not significant on the basis of analysis of variance (ANOVA) analysis. Likewise, there was no significant difference in the invasion potential of $\mathrm{KO}$ cells between normoxic and hypoxic culture.

\section{Expression of Basal Markers Is Reduced in Cultured Knockout Cells}

The expression of markers of the luminal lineage (Troma-I, detecting cytokeratin $8, \mathrm{~K} 8$ ) and the basal lineage (cytokeratins 5 and 14, K5 and K14) by WT and KO cells was evaluated by immunofluorescent staining of nearly confluent cells that were cultured overnight (12 to 14 hours) at normoxia or hypoxia (Figure 3-4). The majority of WT and KO MTECs cultured at normoxia (Figure 3-4A and 3-4E) and hypoxia (Figure 3-4I and 3-4M) stained with antibodies to K8. This was expected because the PyMT model is a luminal-like model of breast cancer. However, fewer KO cells expressed K14 when cultured at either normoxia (Figure 3-4B vs 3-4F) or hypoxia (Figure 3-4J vs 3-4N). Hypoxic culture downregulated K14 expression in both WT and KO cells (Figure 3-4B vs 3-4J and Figure 3-4F vs 3-4N). Overall, fewer KO cells were dual-positive for K8 and K14 (yellow cells), suggesting a reduction in a bipotent progenitor cell population in response to HIF-1 $\alpha$ deletion. The most striking phenotype was that no $\mathrm{K} 5+$ cells could be 


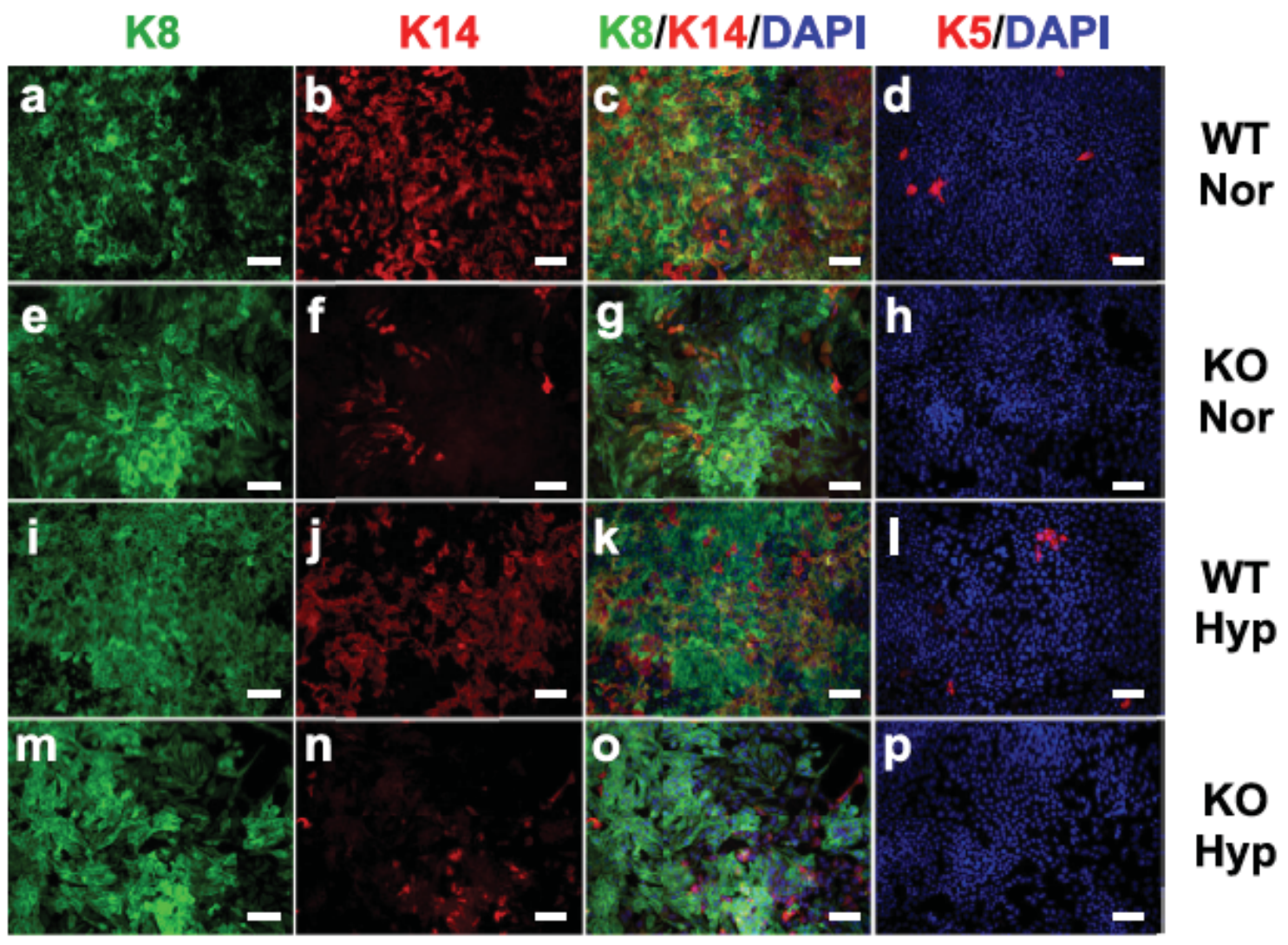

Figure 3-4. Loss of basal marker expression in cultured HIF-1 KO cells. WT or KO cells were plated onto tissue-cultured treated slides and grown to subconfluence, at which time a subset of cells were exposed overnight to hypoxic culture or remained under normoxic culture. Cells were co-stained with Troma-I (K8, green) and K14 (red) (panels a-c, e-g, i-k and m-o) or with K5 alone (panels d, h, 1, p); all slides were counterstained with DAPI. Images were captured at 200x magnification (scale bar indicates $50 \mu \mathrm{m})$. 
detected in KO cells cultured at either normoxia (Figure 3-3D vs 3-3H) or hypoxia (Figure 3-4L vs 3-4P). Hypoxic induction of K5 mRNA has previously been reported in MCF-7 cells, [194]; however, an increase in the number of K5+ cells was not apparent in PyMT WT cells exposed to hypoxia (Figure 3-4D vs 3-4L).

\section{Primary Mammary Tumor Growth Is HIF-1 $\alpha$-Dependent}

To evaluate the contribution of HIF-1 $\alpha$ to primary tumor growth in a syngeneic transplant approach, WT or KO MTECs $(n=50,000)$ were injected into single, cleared inguinal mammary fat pads of FVB/Nj recipient females. Single-side injections were utilized to prevent one tumor from influencing the outgrowth of a contralateral tumor. There was an approximately $60 \%$ decrease in the wet weight and volume of $\mathrm{KO}$ tumors at week 8 post-transplant, when recipients bearing WT tumors required euthanasia in compliance with institutional maximum tumor size recommendations (Figure 3-5A). Moreover, the rate of $\mathrm{KO}$ tumor growth was slower over the entire course of tumor development (Figure 3-5B). When fewer WT or KO cells were utilized to generate mammary tumors (500 cells/gland), there was a more pronounced delay in the ability of $\mathrm{KO}$ cells to form a tumor with a volume $>500 \mathrm{~mm}^{3}$ (Figure 3-5C). The median time to form large tumors increased from 64 days for WT cells to 127 days for KO cells (Figure 3-5C).

To confirm that end point KO tumors did not express HIF-1 $\alpha$, Western blotting was performed using extracts isolated from three randomly selected whole WT or KO tumors. Very low levels of HIF-1 $\alpha$ were detected in $\mathrm{KO}$ end-stage tumors (Figure 3-5D). Residual expression was likely observed because of the presence of stromal components derived from the host recipients, such as tumor-associated macrophages. As expected, the expression of three classic HIF-1 target genes, vascular endothelial growth factor (Vegf), phosphoglycerate kinase 1 (Pgkl) and glucose transporter 1 (Slc2A1 or Glut1), was reduced in KO tumors (Figure 3-5E). However, the gross histopathology of tumors was not affected by deletion of Hifla; all tumors were poorly differentiated, solid adenocarcinomas as previously described in the PyMT-transgenic mouse [124] (Figure 3-6).

Because WT tumors were larger than KO tumors, they also exhibited more extensive necrosis than KO tumors. Because a larger percentage of KO tumor cells were viable, it is not surprising that more Ki67 and caspase 3-positive cells in KO tumors were detected by immunostaining (Figure 3-5F and Figure 3-7). Overall, these changes would be expected to have a zero net effect on KO tumor growth. In addition, as previously reported for late-stage PyMT carcinomas [124], tumors derived from transplanted WT or $\mathrm{KO}$ cells did not exhibit any cells positive for ER $\alpha$ by immunostaining (Figure 3-8). 
Figure 3-5. Deletion of Hifla decreases primary tumor growth.

A. WT or KO cells $(50,000)$ were transplanted into $\mathrm{FVB} / \mathrm{Nj}$ recipients. All tumors were harvested at day 56 to evaluate tumor weight, volume and burden (\% tumor weight/total body weight) ( $\mathrm{n}=10$ recipients/genotype, $p<0.05$, unpaired Student's t-test). B. The growth rate of WT and KO tumors at 50,000 cells input. Best-fit curves were established based on a polynomial fit algorithm in Prism 4.0. Data in A-B are representative of seven independent experiments ( $>60$ recipients/genotype). C. When $500 \mathrm{WT}$ and KO cells are input, the median time to $50 \%$ of recipients to develop tumors $>500 \mathrm{~mm}^{3}$ is 64 days for WT and 127 days for $\mathrm{KO}(\mathrm{n}=14$ recipients/genotype, $p<0.001$, log-rank test). D. Western blotting for HIF-1 $\alpha$ in three independent tumors $\left(500-750 \mathrm{~mm}^{3}\right)$ per genotype; WT and $\mathrm{KO}$ cells that were cultured $6 \mathrm{~h}$ at hypoxia serve as positive and negative controls (CRM, cross-reactive material). E. Mean fold-change \pm S.E.M in expression of HIF-1 targets in $\mathrm{KO}$ tumors as determined by qRT-PCR ( $\mathrm{n}=5$ tumors/genotype). F. An increase in Ki67+ cells in $\mathrm{KO}$ tumors is balanced by an increase in caspase- $3+$ cells $(\mathrm{n}=5$ tumors/genotype, $* p<0.05$, Student's t-test). 
A
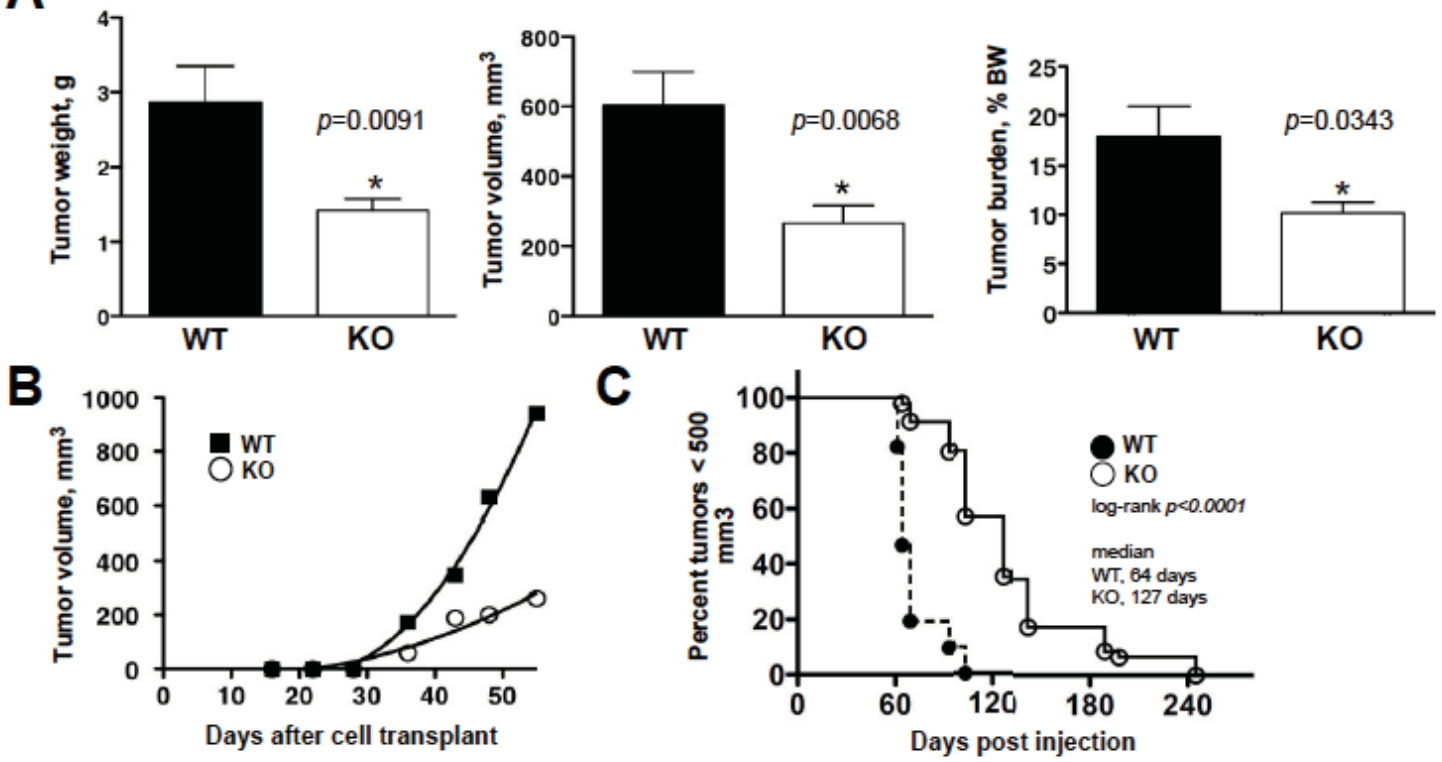

D

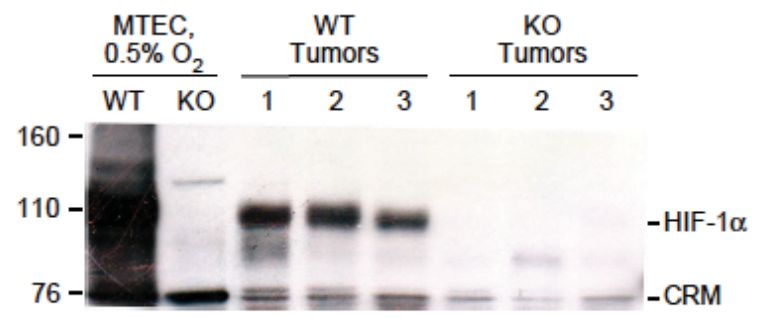

E

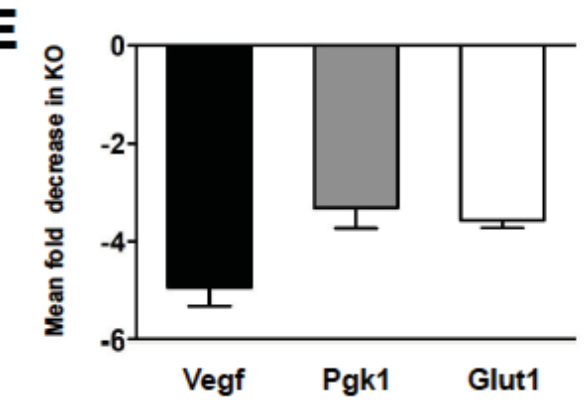

F
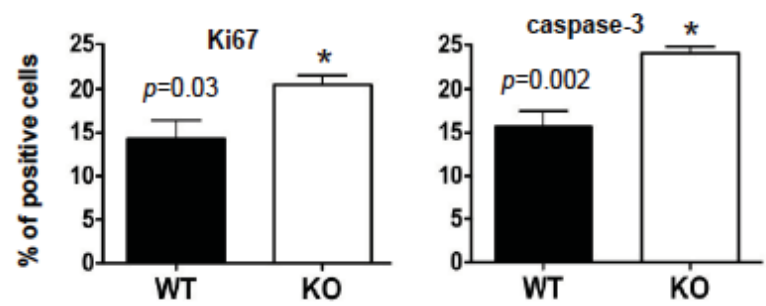


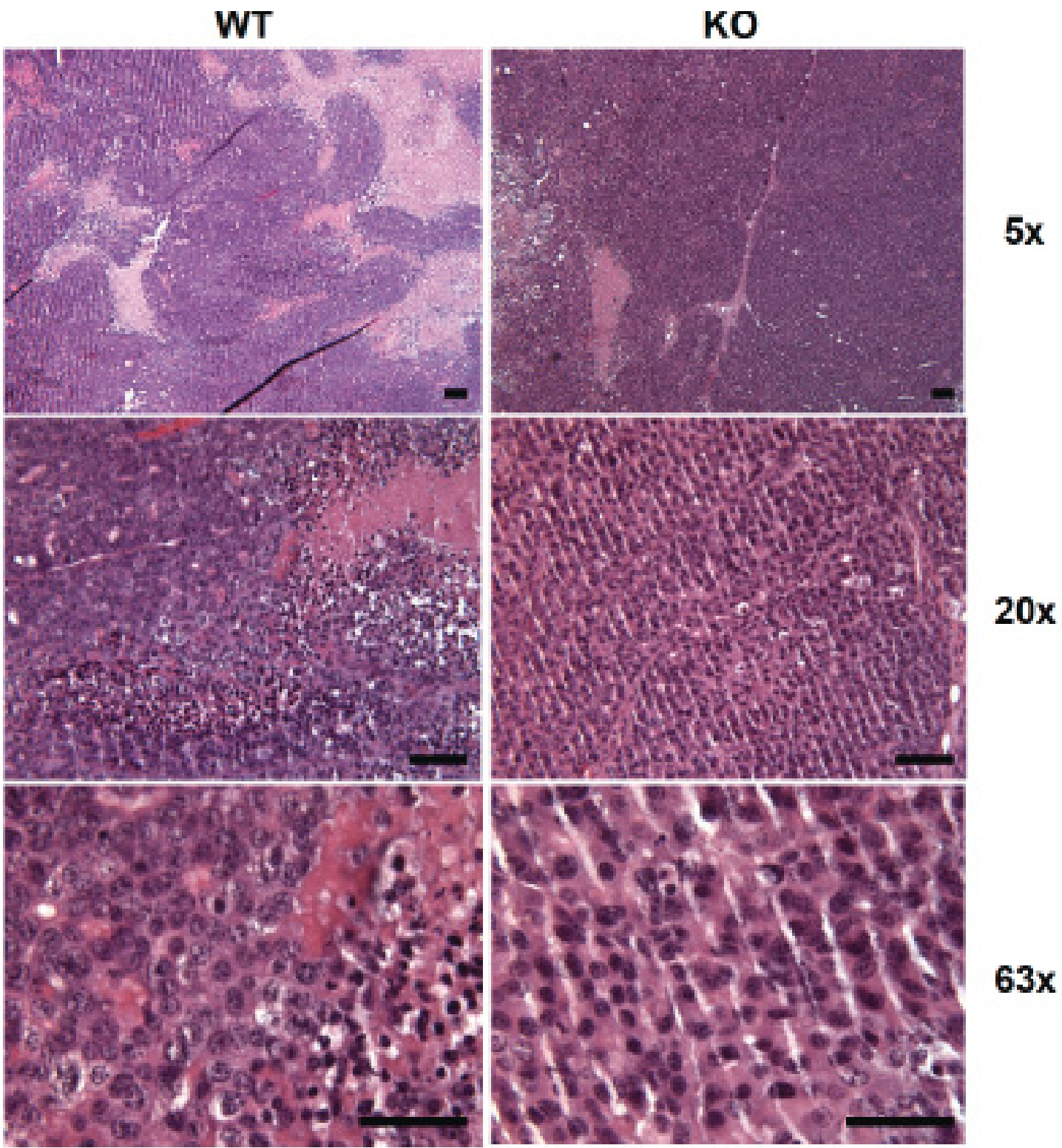

Figure 3-6. Histology of HIF-1 $\alpha$ WT and KO end-stage tumors.

$\mathrm{H} \&$ E-stained sections of end-stage $\left(>750 \mathrm{~mm}^{3}\right)$ tumors derived from WT or KO cells. The WT tumors typically contained more extensive areas of necrosis than KO tumors. One representative tumor per genotype was imaged using the $5 \mathrm{x}, 20 \mathrm{x}$, and $63 \mathrm{x}$ objectives of a Leica DM6000 upright microscope with a SPOT camera, for a final magnification of 50x, 200x or 630x, respectively. Scale bar indicates $50 \mu \mathrm{m}$. 


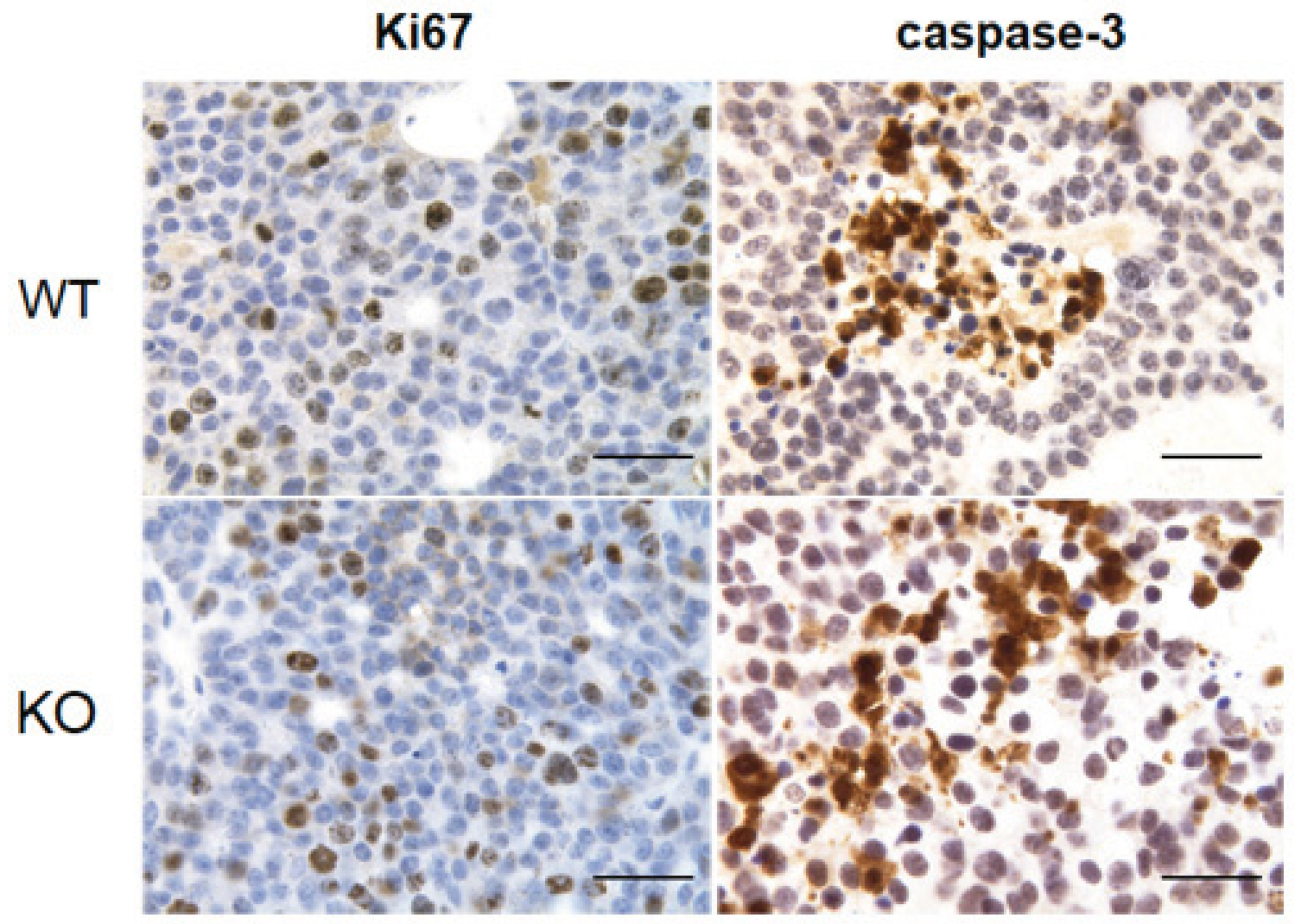

Figure 3-7. Ki67 and activated caspase-3 immunostaining in end-stage PyMT tumors.

Paraffin-embedded, formalin fixed tissue sections from WT and KO tumors $(>500-750$ $\mathrm{mm}^{3}$ ) were immunostained with either Ki67 or activated caspase-3 antibodies and immunoreactive complexes detected using the Vectastain ABC Elite kit and developed with DAB Impact, followed by counterstaining with Harris hematoxylin. Scale bar indicates $100 \mu \mathrm{m}$. 


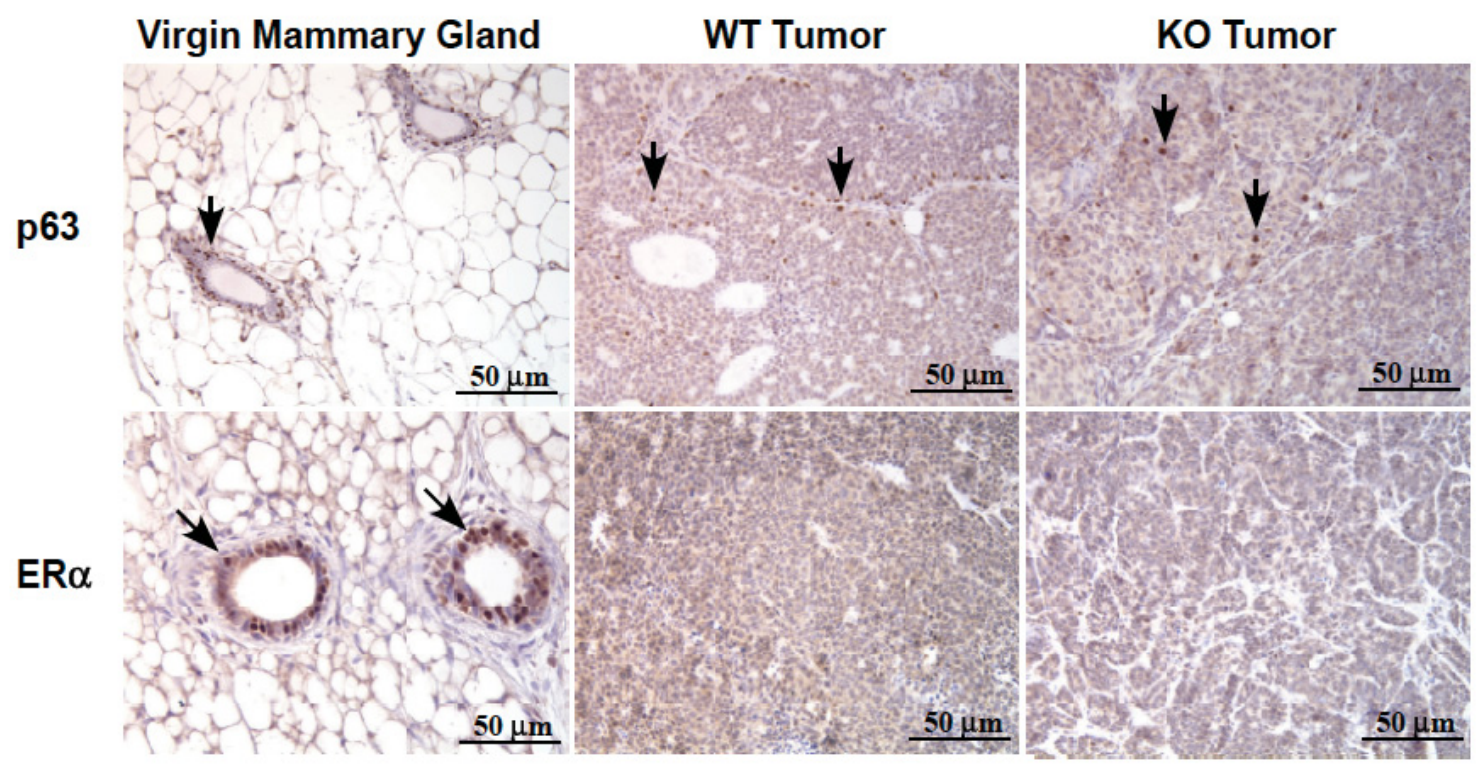

Figure 3-8. Expression of p63 and ER $\alpha$ in WT and KO end-stage PyMT tumors. Representative results of $\mathrm{p} 63$ and $\mathrm{ER} \alpha$ immunostaining in WT and KO tumors ( $\mathrm{n}=4$ /genotype), along with expression observed in normal, virgin mammary glands, which serve as the positive control ( $\mathrm{n}=2$ mice/ 8-weeks of age, FVB strain); 200x magnification, scale bar is $50 \mu \mathrm{m}$. Black arrows point to examples of positive cells. No obvious changes in p63 expression or localization were detected between WT and KO tumors, whereas p63 was located basally within the virgin mammary ductal tree, as expected. No immunoreactivity for ER $\alpha$ was detected in end-stage WT or KO tumors (which were derived from late stage carcinomas of the MMTV-PyMT transgenic mouse), whereas almost all ductal epithelial cells in the virgin mammary gland expressed ER $\alpha$. 


\section{HIF-1 $\alpha$ Promotes an Epithelial-to-Mesenchymal Transition Phenotype and Regulates K5 Expression in vivo}

To determine whether the expression of K5 and $\mathrm{K} 14$ was reduced in $\mathrm{KO}$ tumors as in cultured cells, expression of K8, K14 and K5 was also evaluated by immunofluorescent staining of end-stage tumors harvested from FVB recipients bearing WT or KO tumors (four tumors/cohort). The immunostaining pattern was compared to that observed in tumors harvested from intact PyMT-transgenic mice. In contrast to results obtained in cultured cells, very few cells were dual-positive for K8 and K14 in WT tumors. Furthermore, no cells positive for K5 or K14 were detected in any KO tumors (Figure 3-9A) (four tumors/genotype). In contrast, there were no obvious changes in the expression or localization of $\mathrm{p} 63$ detected by colorimetric immunostaining of paraffin-embedded sections (Figure 3-8).

qRT-PCR analysis of RNA prepared from the same tumors for which sections were prepared for immunostaining revealed that Krt14 (K14) and Krt5 (K5) mRNA levels were reduced in KO tumors by 3.8- and 2.0-fold, respectively. As expected based on the ability of hypoxia to promote an epithelial-to-mesenchymal transition (EMT) phenotype through the Notch pathway [195], multiple core genes in the EMT signature were downregulated in KO tumors compared to WT tumors, including Snail, Slug, Twistl and Fnl (fibronectin), whereas expression of Krt18 (K18) was not affected by HIF-1 $\alpha$ deletion (Figure 3-9B).

\section{Constitutive Hifla Deletion Represses Metastasis and Prolongs Survival}

Initially, metastasis was evaluated in WT or KO tumor recipients by harvesting lungs on the same day as the primary tumors. Because recipients bore a single tumor, in contrast to the transgenic model, in which all ten mammary glands become tumor-laden, very few metastases were present. The lungs from WT hosts exhibited a mean of two micrometastases, and the majority of lungs from $\mathrm{KO}$ hosts were devoid of metastases (Figure 3-10A).

One caveat with regard to interpreting these data is that the tumors developed by the KO cells were 60\% smaller than the WT (Figure 3-10B). Therefore, to compare the effect on lung metastasis when WT and KO tumor volumes were equivalent, the primary tumor was resected during a survival surgical procedure when tumors were at least 1.5 $\mathrm{cm}$ in diameter with a corresponding volume range of 500 to $750 \mathrm{~mm}^{3}$. Animals subjected to survival surgery to resect the primary tumor were divided into two cohorts. In the first cohort, females were euthanized 8 weeks post surgery. WT tumor cells developed large macrometastases visible to the naked eye, as well as several micrometastases visible by microscopic analysis, with a mean of 19 metastases per lung (Figure 3-10B and 3-10A). In contrast, lungs from $\mathrm{KO}$ tumor recipients contained 50\% fewer metastases (Figure 3-10B and 3-10C), which were also smaller overall than the WT metastases. Furthermore, $50 \%$ of host mice transplanted with WT cells were deemed moribund and required euthanasia prior to the 8-week postsurgical period, whereas all 
Figure 3-9. Loss of cells expressing K14 or K5 in HIF-1 KO tumors is accompanied by a reduction in the expression of markers of EMT.

A. Frozen sections were prepared from tumors harvested from PyMT + transgenic mice, or from WT and KO tumors harvested from FVB recipients. All tumors were $\geq 500 \mathrm{~mm}^{3}$ in volume. Sections were co-stained with antibodies to K14 (red) and K8 (green) or K5 (red) alone, and counterstained with DAPI. Images were captured at 200x magnification; the scale bar represents $50 \mu \mathrm{m}$. B. The mean fold-change \pm S.E.M. in gene expression observed in end-stage KO tumors of Krt14 and Krt5 mRNAs, as well multiple markers of EMT, as determined by real-time PCR performed using primers and probes in Table S2 $(\mathrm{n}=5$ tumors/genotype). The mean \pm S.E.M. of biological replicates is graphed. The expression of Snail, Slug, Twist1, Fn1, Krt14 and Krt5 mRNAs was reduced in KO tumors compared to WT tumors, however, the expression of Krt18 varied less than $20 \%$ between genotypes. 


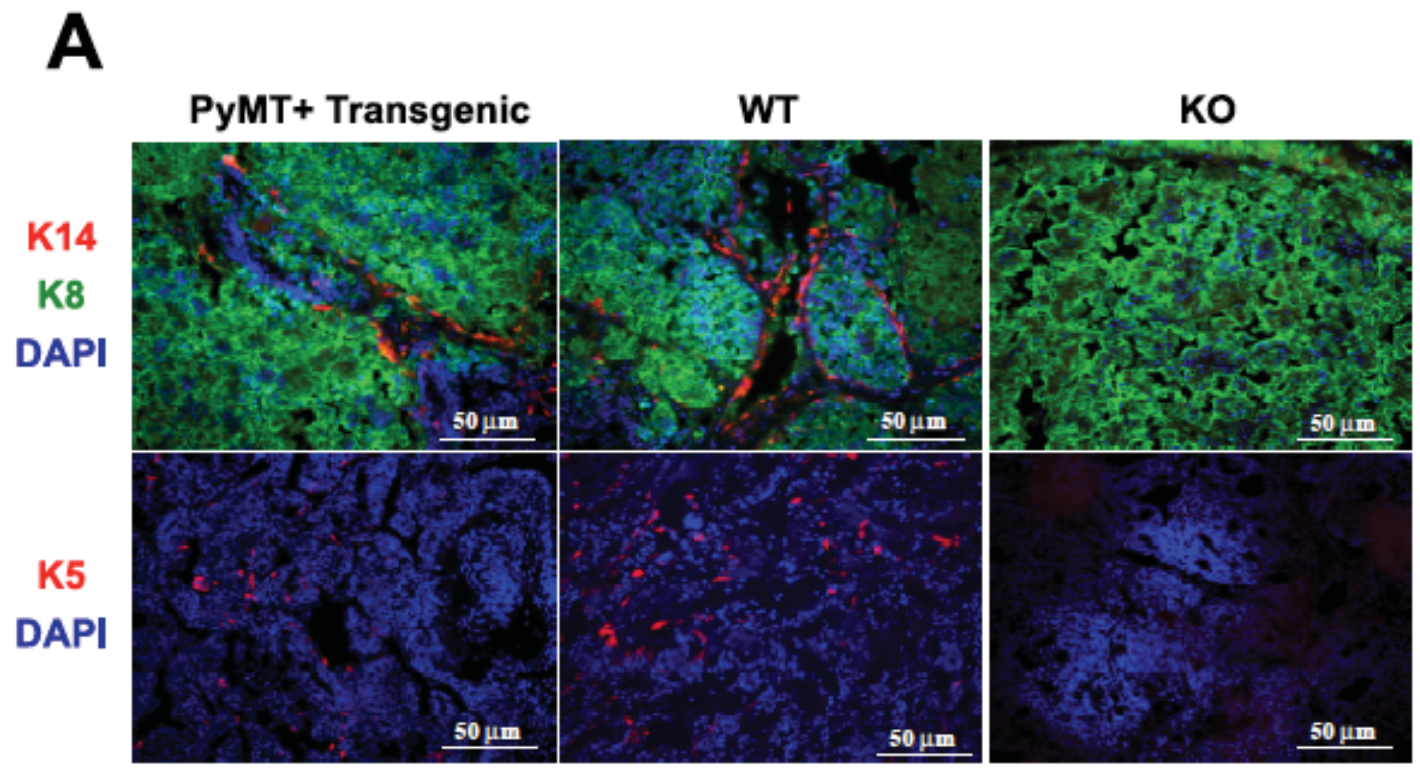

B

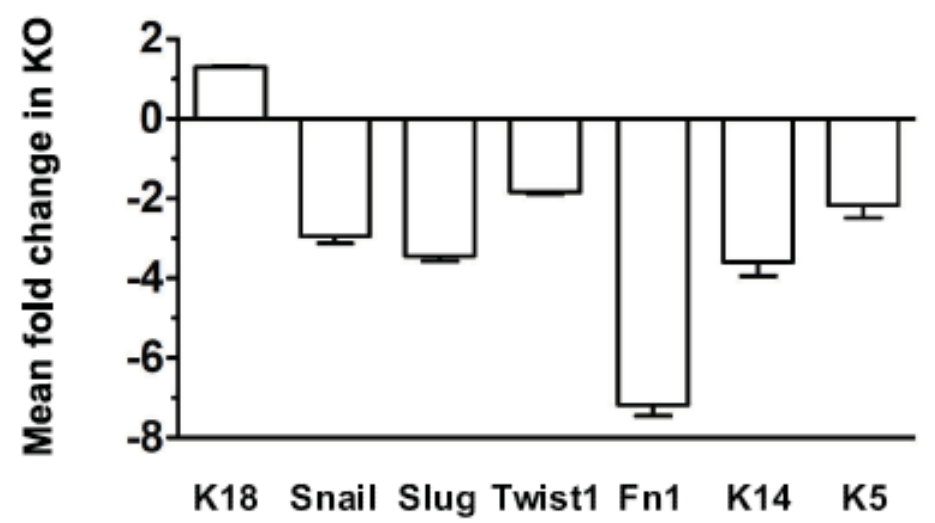


A

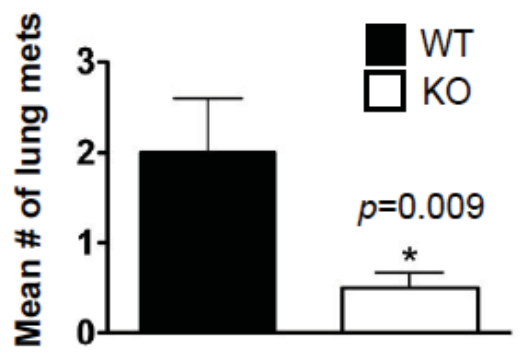

C

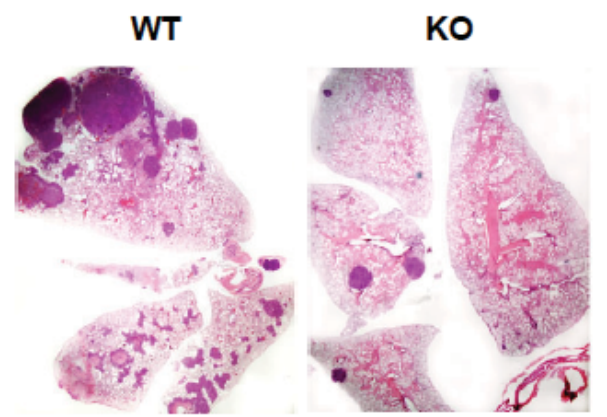

B

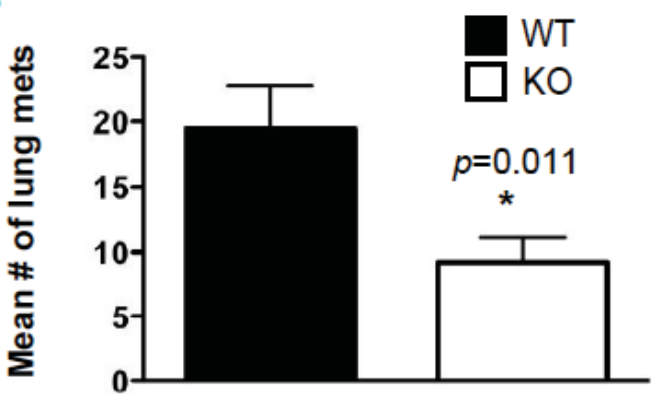

D

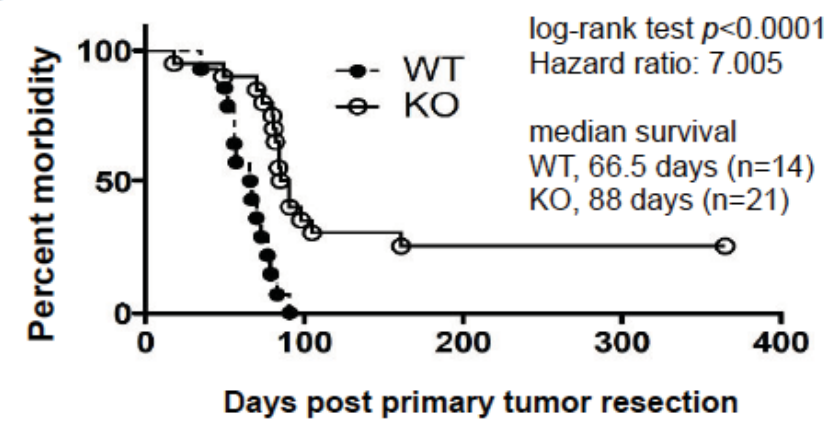

Figure 3-10. HIF-1 $\alpha$ expression in tumor epithelium is required for metastasis. A. Mean number of micrometastases \pm S.E.M observed when lungs are harvested at the same time as the primary tumor $\left(>1,000 \mathrm{~mm}^{3}\right)\left(\mathrm{n}=9 \mathrm{WT}, 14 \mathrm{KO}\right.$ recipients, ${ }^{*} p<0.05$, unpaired student's t-test). B. Enhancement of lung metastases when WT and KO primary tumors are surgically resected at equivalent volumes $\left(500-750 \mathrm{~mm}^{3}\right)$, followed by 8 weeks of survival ( $\mathrm{n}=32 \mathrm{WT}, 22 \mathrm{KO}$ recipients, ${ }^{*} p<0.05$ Student's $t$-test). C.

Representative images of lungs harvested from WT or KO MTEC recipients 8 weeks after tumor resection (50x magnification). D. The impact of HIF-1 $\alpha$ expression in tumor cells on the survival of recipients following tumor resection. A subset of animals subjected to primary tumor resection was allowed to survive until moribund due to metastasis. The morbidity hazard ratio is 3.78 times higher when hosts bear WT tumors $(\mathrm{n}=14 \mathrm{WT}, 15 \mathrm{KO}$ hosts, log-rank test, $p=0.00039,95 \%$ C.I. $=1.53-9.31)$. 
hosts harboring KO cells lived the full 8 weeks. Therefore, although KO MTECs retain the capacity to complete the full metastatic program, metastasis is significantly suppressed.

In the second cohort subjected to the tumor resection paradigm, recipients were allowed to survive until moribund due to lung metastases (Figure 3-10D). Recipients bearing WT tumors succumbed to lung metastases sooner than KO recipients did (Figure 3-10D). The median survival increased from 66.5 days for WT recipients to 88 days for $\mathrm{KO}$ recipients ( $14 \mathrm{WT}$ and $21 \mathrm{KO}$; log rank score, $p<0.001$ ). Furthermore, about $25 \%$ of mice implanted with KO tumors ( 5 of 21 ) lived longer than 1 year and did not develop any lung metastases as evaluated by $\mathrm{H} \& \mathrm{E}$ staining of sections from their lungs.

\section{Deletion of Hif1a Decreases Tumorsphere Formation}

To begin to determine whether HIF-1 $\alpha$ may play a role in regulating breast cancer stem cell activity, single WT or KO cells were placed into tumorsphere culture. All data shown in Figure 3-11 are representative of experiments with several biological replicates. As shown in Figure 3-11A, the mean sphere forming efficiency (SFE) decreased 4.1-fold in the primary, 2.2-fold in the secondary and 2.5-fold in the tertiary generations of spheres derived from KO cells, respectively. The mean SFE among biological replicate experiments varied from $0.06 \%$ to $1.55 \%$ for WT cells and from $0.015 \%$ to $0.85 \%$ for KO cells. Variability in the sphere assay among biological replicates by more than a $\log _{10}$ factor is not unusual [196]. More importantly, SFE within each experiment was always greater for WT cells than for KO cells.

As shown in Figure 3-11, the mean fold enrichment in SFE in WT cells was 4.3fold for the primary generation (range 2.85- to 7.23-fold), 4.1-fold for the secondary generation (range 1.85- to 6.7-fold) and 2.61-fold for the tertiary generation (range 2.1- to 2.79-fold). In each case, the mean fold change was determined on the basis of at least three biological replicates/genotype/generation. Because the WT and KO cells had previously been cultivated in monolayer in the presence of serum, the ability of HIF-1 $\alpha$ to promote SFE was confirmed using tumor cells derived from freshly digested Hifla DF, PyMT + tumors originating in intact transgenic mice.

Following isolation from freshly digested tumors, single cells were immediately exposed in suspension to adeno- $\beta$-gal or adeno-Cre (moi of 80 , for 2 to 3 hours), washed to remove viral particles and then plated at equal density into sphere culture conditions. Spheres derived from the primary generation were digested with trypsin, reexposed to adeno- $\beta$-gal or adeno-Cre in suspension ( 80 moi for 2 to 3 hours) and plated for secondgeneration spheres. Deletion efficiency was determined for each generation by qRT-PCR of genomic DNA isolated from a fraction of cells collected at 72 hours postplating, prior to the formation of large tumorspheres.

In contrast to the established KO MTECs, not all floxed cells exposed 2-3h to 
Figure 3-11. HIF-1 $\alpha$ promotes tumorsphere formation in cultured cells and in freshly isolated tumor cells independent of oxygen tension.

A. Representative experiment in which the mean sphere formation efficiency (SFE) \pm S.E.M. was determined when WT and KO cells were cultured in tumorsphere culture conditions. Tumorspheres $\geq 100 \mu \mathrm{m}$ were scored positive, and the mean SFE (the percentage of cells capable of forming spheres per total number of single cells plated) per experiment ( $\mathrm{n} \geq 12$ wells/genotype; $p<0.05$, unpaired Student's $t$-test) was determined at each of the primary, secondary and tertiary generations of spheres. B. A representative experiment in which the mean primary and secondary SFE \pm S.E.M. was determined when single cells were isolated from mammary tumors of Hifla DF; MMTV-PyMT+ mice followed by immediate adenoviral transduction ( $\mathrm{n} \geq 12$ wells/genotype; $p<0.05$, unpaired Student's $t$-test). C. Comparison of SFE when WT or KO cultured cells were subjected to normoxia or chronic hypoxia. Immediately following plating of single cells, dishes were cultured at normoxia or hypoxia throughout the duration of the experiment, therefore, cells were exposed to chronic hypoxia. The graph presents mean SFE obtained from the secondary generation of spheres ( $\mathrm{n} \geq 12$ wells/genotype). Differences among columns were analyzed by ANOVA, ${ }^{*} p<0.05$. D. The mean fold change in gene expression observed in KO tumorspheres evaluated by qRT-PCR. The grand mean fold change \pm S.E.M. was determined from three independent experiments; cDNA was derived from three preparations of WT and KO secondary spheres grown in 6-well format and pooled at experiment endpoint to prepare total RNA $(\mathrm{n}=3$ pools of spheres/genotype). The mean expression of Notch 4 (N4), Dll1, Hey1, Hey2 and Prom 1 (CD133) was reduced in KO tumorspheres by greater than 1.5 -fold, whereas the mean expression of Notch1, Notch2, and Notch3 (N1-N3), or Vegf did not vary by more than $50 \%$ between genotypes. The decreased expression in $\mathrm{KO}$ tumorspheres is indicated as a negative fold-change. 
A
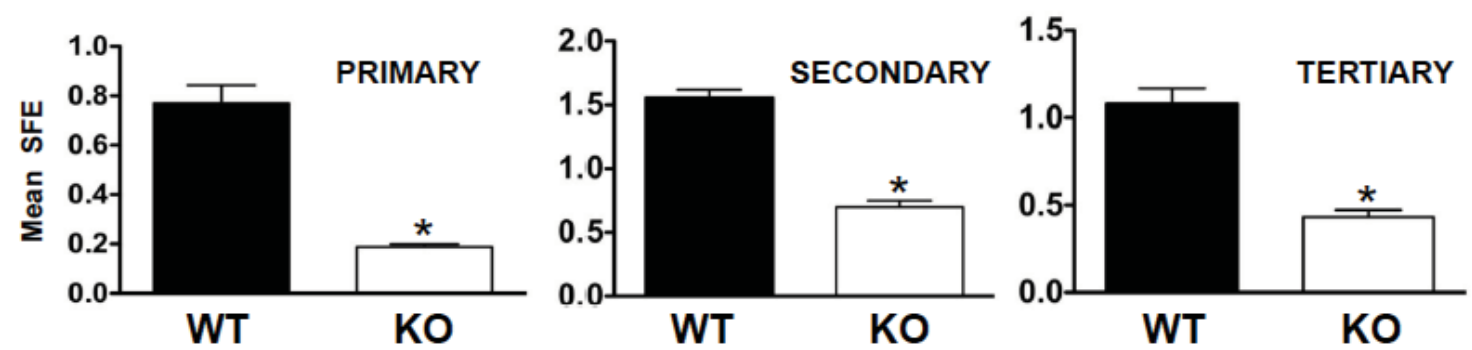

B
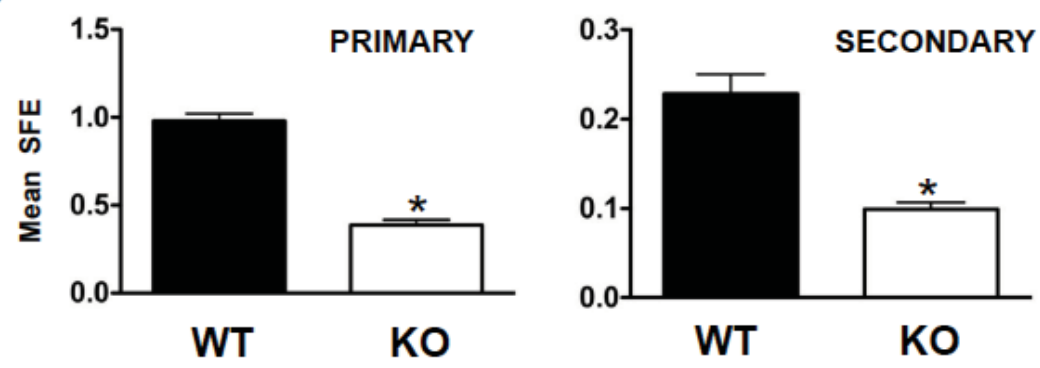

0

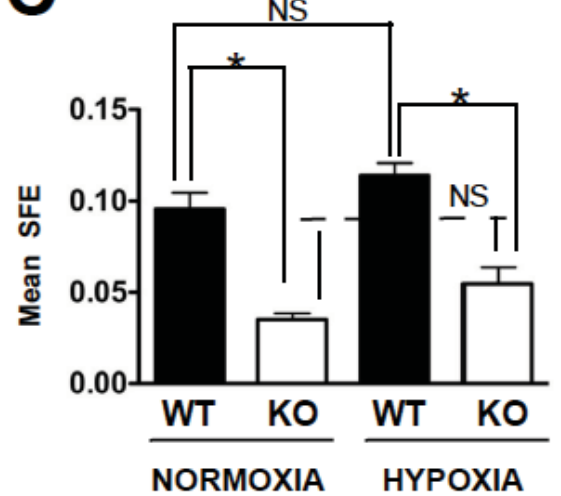

D

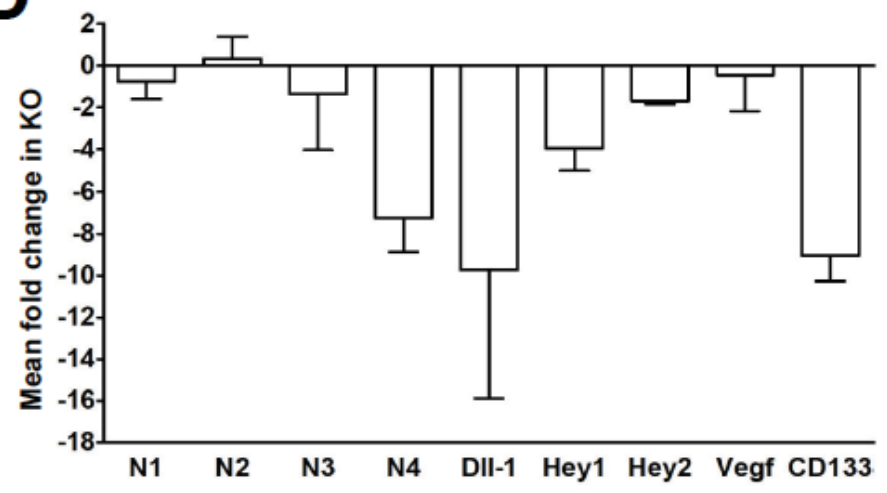


adeno-Cre were deleted for Hifla. A mean deletion efficiency of $71 \%$ or $84 \%$ was observed following the first or second round of transduction with adeno-Cre,respectively. Despite incomplete Hifla deletion primary and secondary SFE was reduced in Hifladeleted cells by 2.53 -fold and 2.30 -fold at the primary and secondary generations, respectively (Figure 3-11B). The mean fold change in SFE between WT and Hifla deleted $(\mathrm{KO})$ cells among three biological replicates of this assay was 5.74-fold for the primary generation (range of 2.53-8.2- fold) and 4.38-fold for the secondary generation (range of 2.30-6.54-fold). These results confirm that the enrichment in SFE observed for WT cells previously cultured in monolayer with FBS was not an artifact due to culture conditions, and suggested that HIF-1 $\alpha$ may promote breast TIC potential in vivo.

Sphere formation was also assayed after culture of WT and KO cells at normoxia or chronic hypoxia. Figure 3-11C presents data from one experiment in which there was a 2.28-fold reduction in KO SFE at normoxia and a 2.1-fold reduction in KO SFE at hypoxia, as compared to WT cells. Among biological replicates the mean fold reduction in KO SFE at normoxia was 3.26-fold (range of 2.20-6.15-fold) and 6.85-fold at hypoxia (range of 2.1 to 9.17-fold). Although there was a trend towards hypoxia increasing WT SFE (mean fold increase in SFE of 1.38, and a range of 1.17- to 1.70-fold), the increase was not statistically significant in any independent experiment by ANOVA. Changes in KO SFE between normoxia and hypoxia were also not significant (mean fold change of 0.94 , and a range of $0.35-2.05$-fold).

To determine if HIF-1 $\alpha$ expression varied in spheres cultured acutely or chronically at hypoxia, HIF-1 $\alpha$ expression was evaluated by western blotting of HS-WCE prepared from WT or KO spheres exposed to prolonged hypoxia ( $\geq 4$ days), or from WT or $\mathrm{KO}$ spheres that were initially grown in normoxic culture, but were then briefly exposed acutely to hypoxia (6 h) prior to harvest (Figure 3-12). In sphere culture conditions, HIF-1 $\alpha$ was barely detectable in WT cells exposed to chronic hypoxia, whereas robust expression of HIF-1 $\alpha$ was observed in WT cells exposed acutely to hypoxia. Therefore, in contrast to data obtained from monolayer cultured cells, EGF was not sufficient to stabilize HIF-1 $\alpha$ expression when cells are grown constitutively at hypoxia in sphere culture.

One caveat of the tumorsphere assay is that spheres may be derived from aggregation of single cells, or even fusions of small spheres. The contribution of cell aggregation to sphere formation was assessed by plating single cells labeled with GFP or mKate at a ratio of $1: 1$ in sphere medium supplemented with $0.5 \%$ methylcellulose, which acts as a physical barrier to impede cellular aggregation. Figure 3-13 shows in these conditions, most spheres were not aggregates of each other, only red cells made red spheres and green cells made green spheres. 


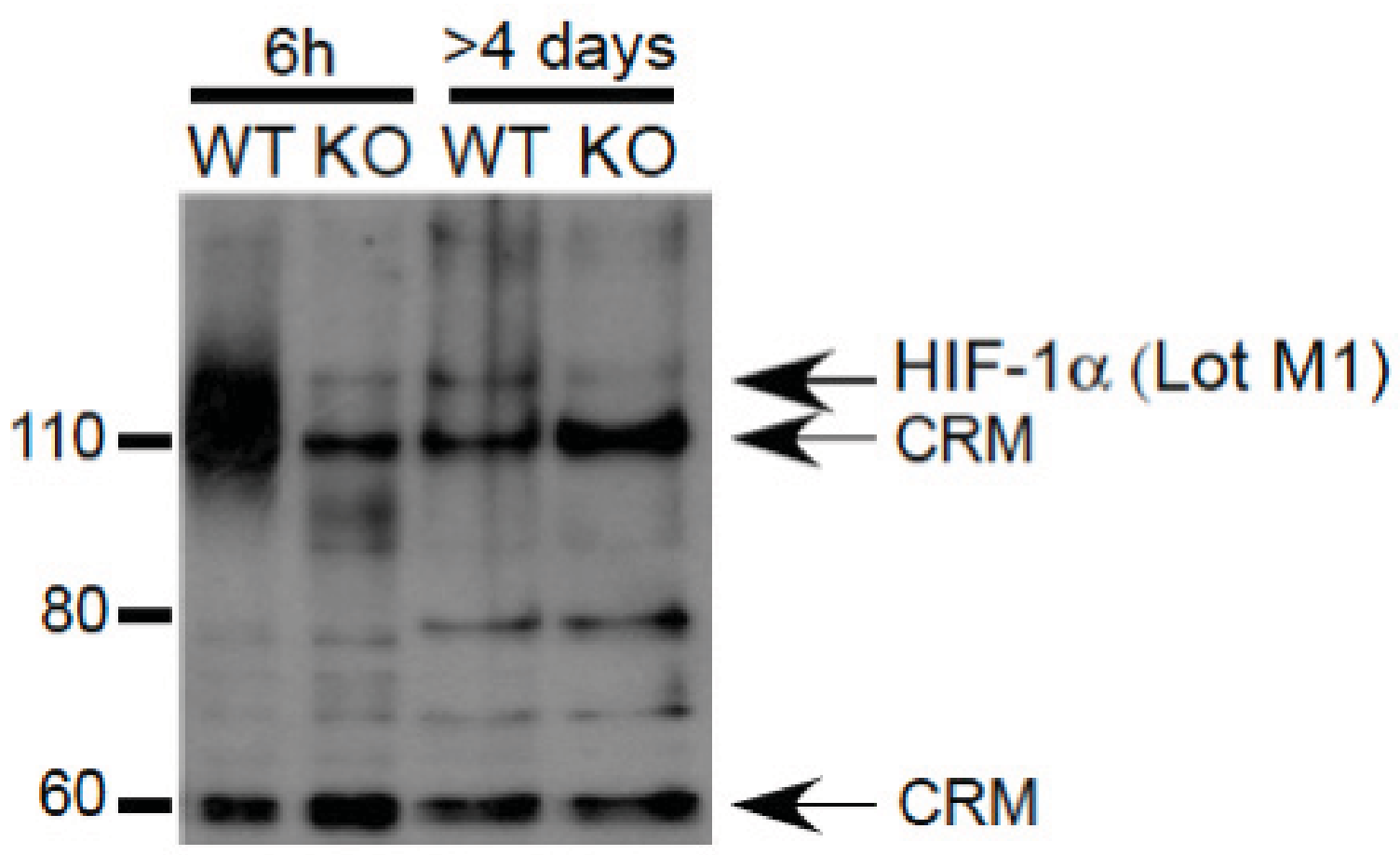

Figure 3-12. HIF-1 $\alpha$ expression in tumorspheres cultured acutely or chronically at hypoxia.

WT or KO tumorspheres were originally derived from single cells plated into ultralow adhesion dishes and cultured at normoxia. A subset of WT and KO tumorspheres (80$100 \mu \mathrm{m}$ in size) were then transferred from normoxic culture to hypoxia $\left(0.5 \% \mathrm{O}_{2}\right)$ for the duration of incubation, which was either acute (6h exposure) or chronic ( $>4$ days exposure). Spheres were then isolated, HS-WCE prepared and HIF-1 $\alpha$ expression levels were evaluated by western blotting (input of $10 \mu \mathrm{g}$ HS-WCE/lane) using lot M1 of Novus 100-479, which produced more cross-reactive material (CRM) bands than previously observed with lot E2. 


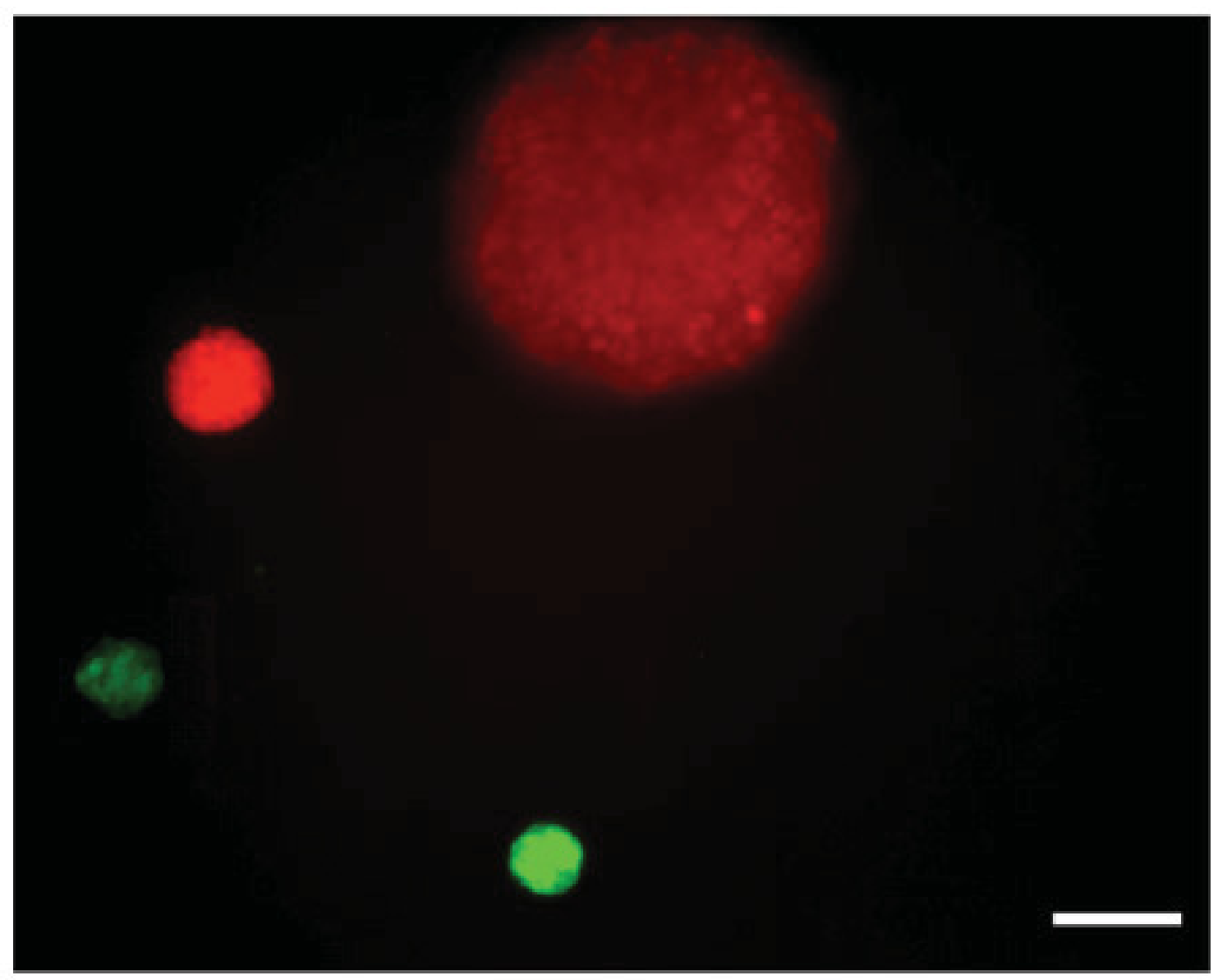

Figure 3-13. Clonal expansion of tumorspheres.

Tumorspheres were derived from PyMT WT single cells labeled with either GFP or mKate mixed at a ratio of 1:1 and plated into ultralow adhesion dishes in 5\% methylcellulose. Scale bar represents $100 \mu \mathrm{m}$. 


\section{HIF-1 $\alpha$-Dependent Regulation of CD133 and the Notch Pathway}

Since CD133, Notch receptors and Notch target genes may each be modulated by the HIF transcription factors $[60,164,197]$, the expression of CD133, genes in the Notch pathway and classic HIF targets were evaluated in WT and KO tumorspheres by qRTPCR. Figure 3-11D presents the mean fold reduction in gene expression observed in $\mathrm{KO}$ tumorspheres. Only genes that were consistently observed to be downregulated across three biological replicate experiments are shown. Expression of Notch4 was downregulated an average of 7.24-fold, whereas expression of the Notch receptorsNotch1, Notch 2 and Notch 3 did not vary by more than twofold. The Notch ligand Delta-like-1 (DlllI) was also downregulated in KO spheres an average of 9.73fold, and the transcription factors Heyland Hey2 were downregulated 3.95-fold and 1.9fold, respectively. The $H E Y$ family of genes has been proposed to function as surrogate markers of Notch activity in multiple human breast cancer cell lines [198, 199].

Although the expression of the Jagged ligands has been reported to be hypoxiainducible in breast cancer cell lines [198, 199], we were unable to confirm a consistent effect of HIF-1 $\alpha$ on the expression of either Jagged 1 or Jagged 2 by tumorspheres. For example, the expression of Jagged 1 was upregulated 3.0-fold in two experiments and downregulated 3.0-fold in a third experiment, whereas the expression of Jagged 2 was observed to be upregulated between 3 - to 10-fold in two experiments and downregulated 3.0-fold in a third experiment (data not shown). In addition, the expression of Dll4, Hes 1 , Hes 2 or Vegf, which is a known direct HIF target gene, did not vary between WT and KO spheres by more than 1.5-fold. The most striking difference in gene expression was observed for Proml (CD133), which was consistently downregulated $>9.0$-fold in KO spheres (Figure 3-11).

On the basis of the qRT-PCR results, the expression of CD133 was then evaluated by immunostaining monolayer-cultured WT and KO MTECs grown at normoxia or hypoxia (Figure 3-14A). Compared to WT cells, CD133-positive cells were not readily detectable by immunostaining of $\mathrm{KO}$ cells following culture at normoxia or hypoxia (Figure 3-14A). These results were validated by flow cytometry following staining of WT and KO cells cultured at normoxia with CD133-PE antibodies. As shown in Figure 3-14B, the expression of CD133 was reduced in KO cells more than 9.0-fold. CD133 expression was also compared by immunostaining tumorspheres derived from WT and KO cells that were grown constitutively at normoxia (Figure 3-14C). As in cultured cells, the expression of CD133 was increased in WT spheres relative to KO spheres (Figure 3-14C), although in contrast to monolayer culture, CD133-positive cells were observed in $\mathrm{KO}$ spheres. Culture conditions influenced the expression of CD133 mRNA levels because expression increased by a mean of 5-fold in WT cells spheres relative to the same passage of WT cells cultured in monolayer in 2\% FBS (data not shown).

To test whether CD133 expression influences SFE, spheres were derived from the $\mathrm{CD} 133^{\text {hi }}$ or CD133 ${ }^{\text {neg }}$ subpopulations of late-stage carcinomas arising in PyMT+ transgenic females. Live Lin ${ }^{\text {neg }}$ single cells were studied by flow cytometry after being 
Figure 3-14. CD133 expression in PyMT MTECs is regulated by HIF-1. A. Sub-confluent WT or KO cultured cells were exposed to acute hypoxia (6h), then immunostained for CD133 (green) and counterstained with DAPI. Images were captured at 200x magnification; the scale bar represents $50 \mu \mathrm{m}$. B. Representative CD133-PE staining profiles of WT (red histogram) and KO (blue histogram) cells cultured at normoxia and subjected to FACS analysis. The isotype only antibody control is also plotted (green histogram). The percentage of cells that were CD133 positive was determined based on the live, singlet, Lin $^{\text {neg }}$ parent population using FlowJo. C. Secondary WT or KO tumorspheres cultured at normoxia were harvested at study endpoint from ultralow adhesion wells, dried onto glass slides, stained with CD133-PE, counterstained with DAPI and imaged by confocal microscopy. The highlighted area (white boxes) shows a higher magnification image, which demonstrates that CD133 is localized to the cell surface/membrane. D. Histogram of CD133-PE (red) in the live, singlet, Lin ${ }^{\text {neg }}$ parent population of cells isolated from tumors that arose in the PyMT transgenic mouse, as compared to the isotype antibody control (green); approximately 6\% of cells were defined in this experiment as CD133 ${ }^{\mathrm{hi}}$. D. A representative experiment showing the mean SFE/well \pm S.E.M of CD133 ${ }^{\text {hi }}$ vs. CD133 ${ }^{\text {neg }}$ sub-populations that were isolated by flow sorting and cultured at normoxia at a density of 10,000 cells/well in 6well format ( $\mathrm{n} \geq 8$ wells/genotype, unpaired Student's $t$-test). The SFE was determined as the percentage of cells capable of forming spheres per the total number of single cells plated. 
A

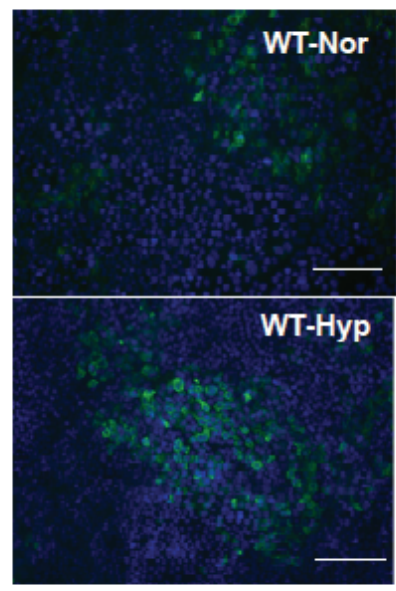

C
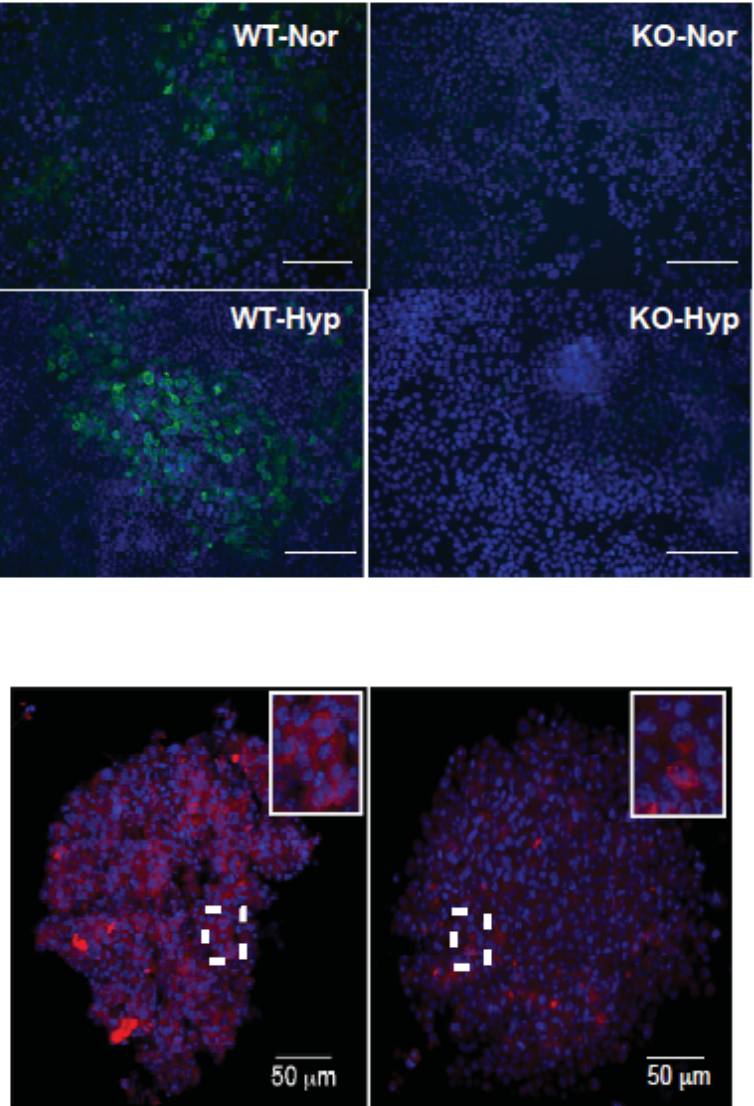

E

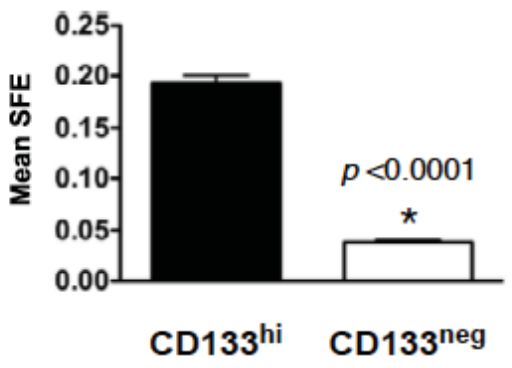

B

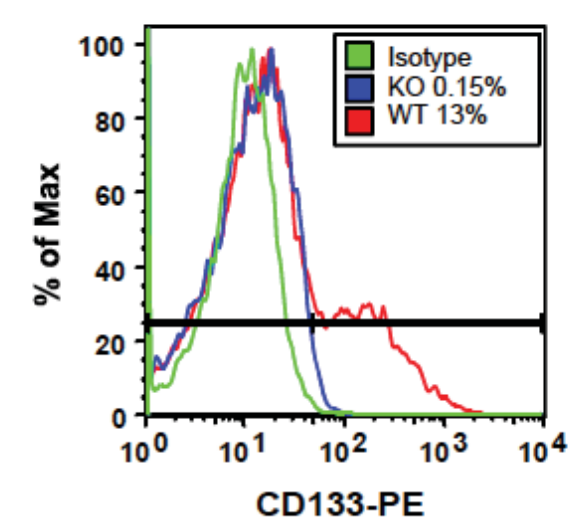

D

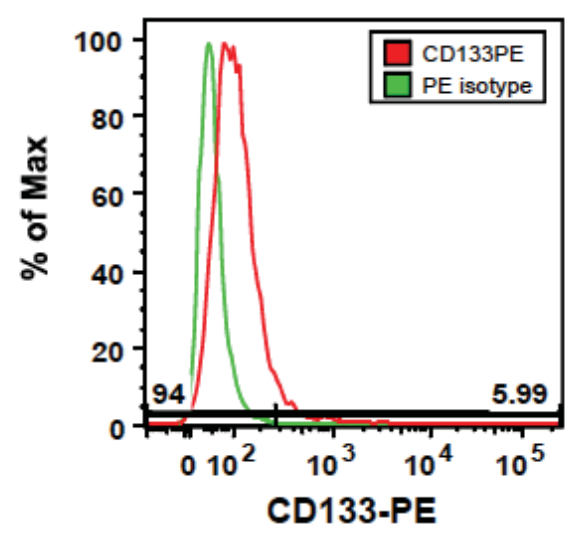


stained with CD133-PE. Among replicate experiments, the tumor epithelial cells identified as highly positive for CD133-PE ranged from 5\% to $12 \%$. A representative histogram is presented in Figure 3-14D, showing that $5.99 \%$ of cells were isolated as the CD133 ${ }^{\text {hi }}$ fraction. The mean increase in SFE in the CD133 ${ }^{\text {hi }}$ subpopulation compared to the $\mathrm{CD} 133^{\text {neg }}$ fraction among replicate experiments was $>4.0$-fold (a representative experiment is shown in Figure 3-14E), suggesting that CD133 may be a useful cell surface marker to enrich for TICs in the PyMT model.

\section{HIF-1a Promotes Tumor-Initiating Cell Frequency in vivo}

On the basis of the dramatic differences in growth rates of WT and $\mathrm{KO}$ tumors and the reduction in SFE by HIF- $1 \alpha-\mathrm{KO}$ cells in tumorsphere assays, the ability of WT and $\mathrm{KO}$ cells to form tumors in recipients under limiting dilution transplantation conditions was evaluated. To permit estimation of TIC frequency due exclusively to loss of HIF- $1 \alpha$ activity, TIC potential was compared using unsorted WT or KO MTECs to avoid the physical stress encountered during sorting that reduces cell viability and therefore affects the calculation of TIC frequency [119]. The ability of WT or KO MTECs to form a palpable tumor mass $>5 \mathrm{~mm}$ was evaluated using a broad range of cell dilutions (10 to 500 cells: 10, 25, 50, 100, 200 and 500) (Table 3-1). At each cell density evaluated, there was a statistically significant enrichment in TIC activity in WT cells, which was indicated by the percentage of $\mathrm{FVB} / \mathrm{Nj}$ recipients positive for a palpable tumor (Figure 3-15A and Table 3-1). ELDA analysis [189] revealed a 35-fold reduction in TIC frequency from KO to WT cells at day 36 posttransplant (Table 3-1). Data were also statistically significant on the basis of the Fisher's exact test (Table 3-1). The difference in TIC potential remained statistically significant at day 62 posttransplant in the fold enrichment of TIC frequency at day 62 may be that the data from the 500- and 200-cell recipients were excluded from the ELDA analysis [127]. All recipients in these two cohorts were euthanized at day 36 to compare the histological and gene expression profiles of early-stage lesions. Notably, WT recipients in the 50- and 100-cell input cohorts required surgical intervention or euthanasia based on tumor volume by day 62 , whereas no intervention was required for KO recipients until day 96 posttransplant [127].

The mean volume of $\mathrm{KO}$ tumors derived under limiting dilution conditions was reduced, and this difference was maintained over several months. None of the KO tumors derived from transplantation of 50 or fewer cells exceeded a volume of $100 \mathrm{~mm}^{3}$ by day 62 (Figure 3-15B). By day 112 posttransplant, 96\% of recipients that received $10 \mathrm{WT}$ cells had developed measurable tumors, whereas $55 \%$ of recipients that received $10 \mathrm{KO}$ cells had not. The mean tumor volume among the tumor-positive 10 -cell $\mathrm{KO}$ recipients was $<250 \mathrm{~mm}^{3}$. The difference in TIC potential in the 10 -cell cohort remained significant until day 244, when all animals were euthanized (Supplementary Data, [127]).

HIF-1 $\alpha$-dependent changes in gene expression were evaluated in early-stage WT and $\mathrm{KO}$ masses ( 75 to $200 \mathrm{~mm}^{3}$; five tumors per genotype). Similar to results obtained from spheres, Notch4, Heyl and Hey2 mRNA levels were decreased in KO tumors 2.25-, 4.27- and 2.3-fold, respectively (Figure 3-15C), whereas Notch1-Notch3 mRNA levels 
Table 3-1. Estimation of TIC frequency of HIF-1 $\alpha$ WT and KO MTECs.

\begin{tabular}{lccc}
\hline \multirow{2}{*}{$\begin{array}{c}\text { Number of Cells } \\
\text { Injected }\end{array}$} & HIF-1 $\boldsymbol{\alpha}$ WT & HIF-1 $\boldsymbol{\alpha}$ KO & $\begin{array}{c}\text { Fisher's Exact } \\
\text { Test }\end{array}$ \\
\cline { 2 - 3 } 500 & $78 \%(7 / 9)$ & $0 \%(0 / 8)$ & $p=0.0023$ \\
200 & $90 \%(9 / 10)$ & $10 \%(1 / 10)$ & $p=0.0011$ \\
100 & $77 \%(10 / 13)$ & $9 \%(1 / 11)$ & $p=0.0003$ \\
50 & $69 \%(11 / 16)$ & $6 \%(1 / 16)$ & $p=0.0006$ \\
25 & $30 \%(6 / 20)$ & $0 \%(0 / 20)$ & $p=0.0202$ \\
10 & $35 \%(8 / 23)$ & $0 \%(0 / 22)$ & $p=0.0038$ \\
Estimated TIC & & & \\
Frequency by & $1 / 82$ & $1 / 2915$ & \\
ELDA (95\% C.I.) & $(1 / 57-1 / 117)$ & $(1 / 925-1 / 9193)$ & $p=5.79 \mathrm{e}^{-2} 21$ \\
\hline
\end{tabular}


Figure 3-15. HIF-1 $\alpha$ regulates tumor-initiation potential in vivo.

A. Evaluation of the percentage of recipients (number of recipients positive for a palpable mass/number of recipients successfully transplanted x100) that developed a tumor mass by day 36 post-injection of unsorted WT and KO tumor cells. The asterisks indicate a significant difference between genotypes for a given cell density by Fisher's exact test. B. Comparison of WT and KO mean tumor volume \pm SEM over time when 10, 25 or 50 cells are injected into the mammary fat pad. C. The mean fold change \pm S.E.M. in gene expression profiles of early-stage tumor masses was evaluated by qRT-PCR; all genes displayed were down-regulated in KO tumors as compared to WT tumors $(n=4$ tumors/genotype/experiment). 
A

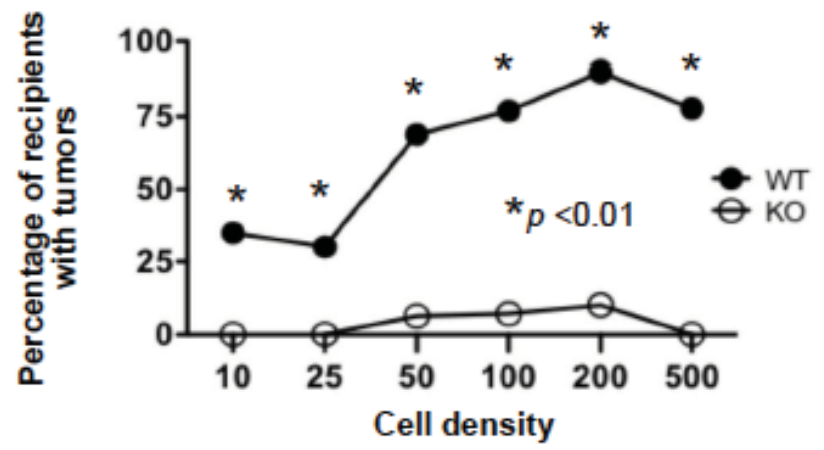

B

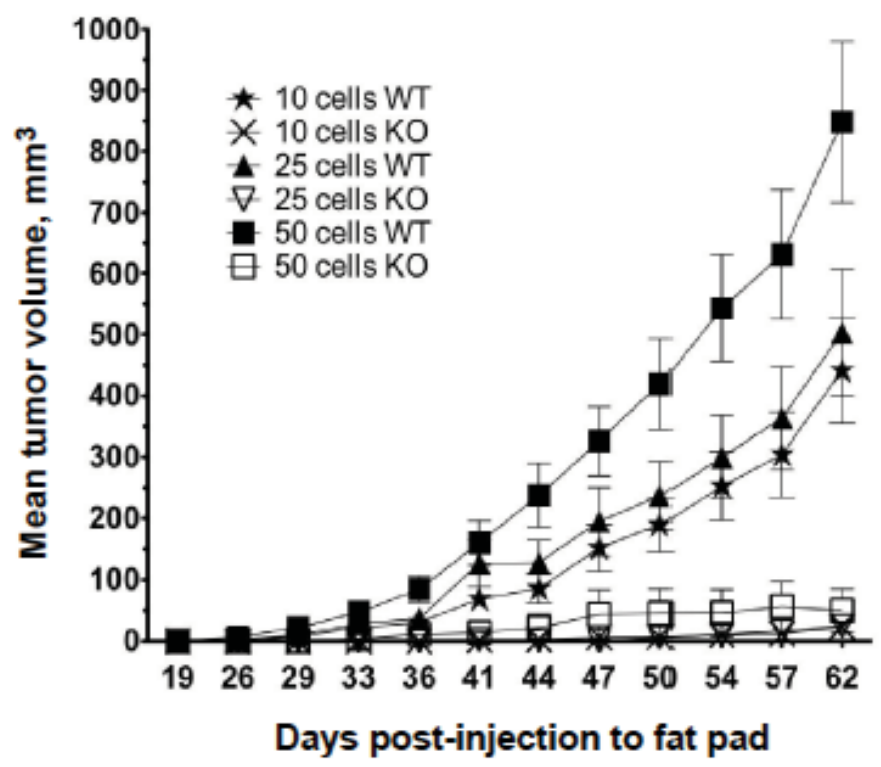

C

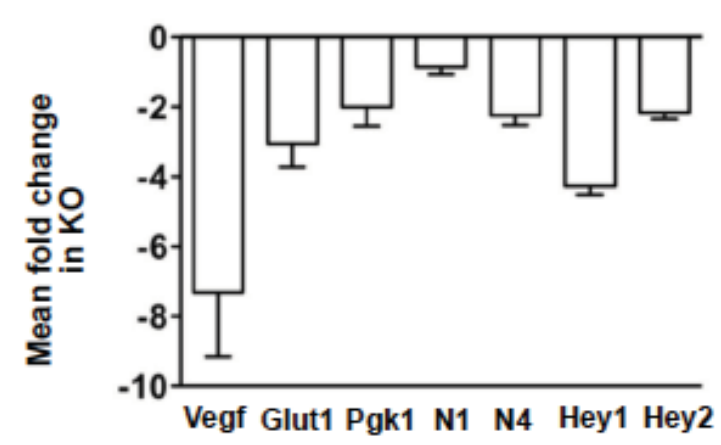

D

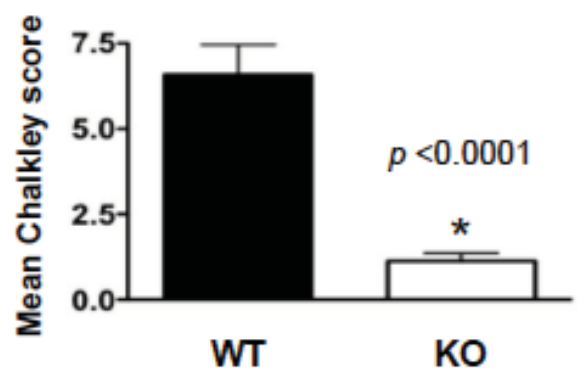


decreased by less than $50 \%$. All other genes in the Notch pathway that were previously found to be differentially expressed in spheres were not differentially expressed among WT and KO tumors, except for Vegf, which was downregulated by a mean of 7.3-fold. As observed in end-stage tumors, WT and KO Glut 1 and Pgkl mRNA levels were reduced in tumors $<200 \mathrm{~mm}^{3}$.

No gross changes in WT or KO tumor histopathology were observed in recipients transplanted with 500 tumor cells. None of the KO masses within this cohort were detectable by manual palpation, but microscopic analysis of $\mathrm{H} \&$ E-stained sections revealed lesions $<5 \mathrm{~mm}$ in five of eight recipients. Since $V e g f$ mRNA was differentially expressed, microvessel density was evaluated by Chalkley scoring of sections immunostained with anti-CD34 antibodies. Decreased Vegf mRNA levels corresponded with a concomitant decrease in mean Chalkley score in KO tumors (Figure 3-15D). In summary, deletion of Hifla reduced TIC potential as well as neoangiogenesis.

\section{Discussion}

Although the role of hypoxia and the HIF-1 $\alpha$ transcriptional response in promoting tumor progression and metastasis is well-established, the direct contribution of the HIF family to the regulation of TICs in breast cancer is unknown. HIF-1 $\alpha$ rather than HIF-2 $\alpha$ is believed to be the predominant regulator of the hypoxic response in breast cancer [22]; therefore, we sought to determine the effect of Hifla deletion in the MMTVPyMT model of breast cancer. In this study, by using an ex vivo genetic deletion approach, we generated constitutive HIF-1 $\alpha-\mathrm{KO}$ and control (WT) MTECs isolated from a pool of MMTV-PyMT tumors. One distinct advantage of this approach is that the MTECs are transplantable to immunocompetent hosts, preserving any host-derived effects on TIC potential following limiting dilution transplantation, in contrast to the xenograft of human cells into immunocompromised mice. Herein we show for the first time that HIF-1 $\alpha$ positively regulates TIC activity in breast cancer as suggested by sphere formation assays in vitro and validated through limiting dilution transplantation of WT and $\mathrm{KO}$ cells.

Additional advantages of the exogenous transduction and transplantation approach versus crosses to MMTV-Cre-transgenic mice include avoiding the mosaic nature of MMTV-Cre expression, because not all epithelial cells harboring floxed alleles of Hifla undergo recombination [50], and avoiding the use of a mixed genetic strain background by utilizing mouse models backcrossed to a single strain (FVB/ Nj). Because Hifla deletion impairs cell proliferation [193], an effect we also observed in cultured KO MTECs (Figure 3-2B), incomplete recombination could permit nontargeted cells to outgrow the recombined (KO) cells. Additionally, it had not previously been assayed whether the lung metastases originating in the MMTV-Cre-derived conditional $\mathrm{KO}$ females were derived from the recombined tumor cells or were generated from cells that had escaped recombination [126]. In addition, the prior use of a mixed strain background may have introduced genetic modifier effects that could influence tumor incidence and lung metastasis. In particular, both tumor burden and lung metastasis in the 
MMTV-PyMT model are enhanced on the FVB/N background as compared to C57BL/6 [200].

In agreement with the findings of previous studies, our study results confirm that HIF-1 $\alpha$ promotes the growth of MTECs cultured at hypoxia and enhances lung metastasis in vivo [126]. However, there were some differences observed between the two model systems. For example, we found that deletion of Hifla repressed the growth of cells at normoxia as well as at hypoxia (by 48 hours of culture). In addition, the magnitude of the decrease in KO cell invasion was similar, regardless of whether cells were cultured at normoxia or hypoxia, whereas Liao et al. reported a difference only at hypoxia [126]. No statistically significant increase in invasion potential between WT cells cultured at normoxia and hypoxia was observed, as reported previously [126]. In contrast to observations from either PyMT model system in which Hifla was deleted, short hairpin RNA-mediated knockdown of HIF $1 A$ in MDA- MB-231 breast cancer cells does not significantly change cell number at either normoxia or hypoxia $\left(1 \% \mathrm{O}_{2}\right)$ [72]. It is possible that the effects of loss of HIF-1 $\alpha$ activity on PyMT cell growth in monolayer culture would be attenuated if cells were cultured at higher serum levels since MDA-MB231 cells were cultured in medium containing 10\% FBS whereas PyMT cell lines were cultured in medium containing $2 \%$ or $5 \%$ FBS. We further demonstrate that primary tumor growth and survival from distant metastases are dependent upon epithelial cell intrinsic HIF-1 $\alpha$ expression. In stark contrast to results obtained previously [126], we found that HIF-1 $\alpha$ plays a significant role in the control of primary PyMT-induced mammary tumor growth under either standard (50,000-cell input) or limiting dilution cell transplantation conditions (50- to 500-cell input). The cause of the control of net mammary tumor growth by HIF- $1 \alpha$ is unclear, although our data suggest that HIF-1 $\alpha$ dependent control of TIC activity may be a primary mechanism driving tumorigenesis. The significant changes in $V e g f$ mRNA expression and microvessel density observed in early-stage KO tumors may also contribute to the phenotype by further restricting tumor growth once tumors are initiated. In agreement with our observations, a HIF-1 $\alpha$ dependent effect on tumor growth was demonstrated recently in both MDA-MB-231 and MDA-MB-435 breast cancer xenografts $[71,72]$.

Characterization of the expression of luminal (K8/K18) and myoepithelial (K14/K5) lineage markers in WT and KO PyMT cells and tumors revealed that fewer KO cells than WT cells coexpressed K8/K14, primarily due to loss of K14-positive cells. These results suggest that loss of HIF-1 $\alpha$ activity corresponds with a reduction in bipotent, stemlike cells in PyMT tumors. The most dramatic phenotype was the absence of K5-positive cells in KO cultures and the loss of both K14- and K5-positive cells in KO tumors. These data are of interest, given the stratification of triple-negative breast cancers (TNBCs) into non-basal-like or basal-like subtypes, based on the expression of both K5 and epidermal growth factor receptors (EGFRs) in tumors within the basal-like subtype [201].

TNBC patients with a basal-like classification have shorter disease-free or overall survival $[202,203]$ and tumors from TNBC patients with metastatic disease exhibit higher levels of K5 and EFGR [204]. A potential influence of HIF-1 $\alpha$ on the ability of the 
K5 and EGFR biomarkers to predict disease-free survival in basal-like TNBC is intriguing in light of our observations that, in cultured cells, EGF stabilizes HIF-1 $\alpha$ expression at normoxia and further potentiates hypoxia-inducible expression of HIF-1 $\alpha$. Although the PyMT model is classified as a luminal-like cancer [125], late-stage tumors such as the ones utilized to generate HIF-1 $\alpha$ WT and KO cells are ER- [124]. Subpopulations of both WT cells and WT tumors were positive for K5.

It has also been observed in luminal breast cancer cell lines (T47D and MCF7), which contain subpopulations of ER-/PR-/K5+ cells, that the K5+ cells are enriched for TIC activity and are resistant to conventional chemotherapies compared to ER+/PR+/K5cells [205]. Of additional relevance to our results regarding the decreased expression of K5, K14 and markers of EMT, including Slug, by KO tumors is that hypoxia-dependent elevation in K5 mRNA levels occurs in a SLUG-dependent manner in MCF7 cells [194]. Furthermore, a statistically significant correlation was found to exist between tumors with high SLUG expression and PROM1 (CD133) expression. These tumors also expressed high levels of carbonic anhydrase IX (CAR9), which is a known HIF-1 target gene [194].

Recent whole-genome expression profiling of breast cancers has revealed that the hypoxic response (predominantly through HIF-1 $\alpha$ ), the EGFR and signal transducer and activator of transcription 3 (STAT3) pathways are positively correlated together in TNBCs as compared to luminal cancers [92]. In support of a functional association between the HIF-1 $\alpha$ and EGFR pathways in TNBCs, MDA-MB-231 cells treated with gefitinib were found to exhibit downregulation of HIF-1 $\alpha$ transcriptional activity that corresponded with decreases in cell viability and migration, whereas resistance to cetuximab or lapatinib therapy was hypothesized to be due to the inability of either drug to downregulate HIF-1 $\alpha$ activity [205]. Taken together, these observations suggest that targeting the HIF pathway may be beneficial to TNBC patients, particularly those patients diagnosed with basal-like TNBC.

Based upon the HIF-1 $\alpha$-dependent control of CD133 expression in MTECs and tumorspheres, as well as the enrichment of sphere formation in the $\mathrm{CD} 133^{\mathrm{hi}}$ versus $\mathrm{CD} 133^{\text {neg }}$ populations, we have identified CD133 as a cell surface marker that may enrich for TICs in the PyMT model. Antibodies to epitope 2 of CD133 (CD133/2) have been utilized extensively to enrich for TICs in other solid tumors, particularly in human colon cancer and gliomas. In contrast, in the normal mammary gland, CD133 is expressed by differentiated ER+ luminal cells, and CD133+ cells exhibit lower regenerative capacity than CD133- cells [206]. Notably, deletion of Prom 1 in a knockout mouse model did not impair the regenerative capacity of the normal mammary gland, but did reduce ductal branching during morphogenesis by increasing the ratio of luminal to basal cells [146]. Yet, in the NKI 295 data set, PROMI expression levels were found to be lower in ER $\alpha^{+}$ tumors than in ER $\alpha$ - tumors [146]. The association of HIF-1 $\alpha$ and PROMI expression in ER- breast cancers is not surprising, given that hypoxia is a potent stimulator of ER $\alpha$ degradation $[207,208]$.

In contrast to observations in the normal mammary gland, Meyer and colleagues have recently shown that, in the context of ER- breast cancers, CD133 enriches for TICs 
when used in conjunction with CD49f (integrin a6) and CD44 [209]. Specifically, the CD49f+/CD44+/CD133 ${ }^{\text {hi }}$ population identified tumor cells with enriched sphere-forming and xenografting potential. Likewise, CD133 has also been shown to enrich for TICs in the Brcal conditional mouse [120]. That a marker of a differentiated normal mammary epithelial cell could enrich for cells with TIC activity in the context of breast cancer is also supported by recent evidence from multiple laboratories that, in $B R C A 1$ basal-like tumors, the TIC population arises from the luminal lineage [210-212]. Interestingly, basal-like BRCA1 tumors have previously been shown to overexpress HIF-1 $\alpha$ and higher HIF-1 $\alpha$ levels have been found to be correlated with decreased disease-free survival [213, 214]. Moreover, Proia et al. found that SLUG promoted a basal-like phenotype before and after transformation in BRCAl tumors [212], which is consistent with our observation that expression of Slug decreased more than threefold in HIF-1 $\alpha$-KO PyMT tumors that did not express the basal markers K5 and K14.

Furthermore, accumulating evidence suggests that differentiated cells (lineagerestricted progeny) may reacquire stem cell-like potential and tumor-initiating capacity rather than follow a strict linear hierarchy as originally proposed for the normal mammary gland. The plasticity involved in breast stem cell biology is emerging. Two independent groups have observed the spontaneous conversion of non-stem cells into stem cells $[215,216]$. Likewise, the results of recent lineage-tracing experiments by researchers in the Blanpain laboratory have challenged the requirement for a bipotent stem cell in the postnatal normal mammary gland [217]. Under the limiting cell conditions routinely used to document the regenerative capacity of a given cell population, these authors found that the disruption of the normal luminal-tomyoepithelial cell ratio is sufficient to stimulate a unipotent myoepithelial progenitor cell to reacquire a bipotent progenitor activity that is normally restricted to the embryonic gland [217]. Yet, how, or if, this model derived from lineage-tracing experiments can be applied to TICs during breast tumorigenesis remains unknown.

One mechanism of HIF-1 $\alpha$-dependent control of TIC may be through regulation of the Notch pathway. Interactions of HIF-1 $\alpha$ with the Notch intracellular domain enhances the regulation of Notch transcriptional targets, such as the $H E Y$ genes, and promotes EMT in breast cancer $[60,158,218]$. In addition, in breast cancer, NOTCH1 and NOTCH4 have been positively correlated with stemness, and blocking antibodies to NOTCH4 reduces mammosphere formation [218]. In KO tumorspheres and early-stage KO tumors, decreased expression of several members of the Notch pathway, particularly Notch4 and Heyl, was observed. Changes in Notch4 are of particular interest because previous studies have shown that blocking NOTCH4 receptor activity inhibits tumor formation of xenografted breast cancer cells, whereas blocking NOTCH1 has less of an effect [218]. Although a positive correlation between hypoxia and NOTCH3 expression was previously described in breast cancer [169], no HIF-1 $\alpha-$ dependent changes in Notch3 were observed in our studies.

HIF-1 $\alpha$-dependent effects on sphere formation efficiency in vitro and TIC activity in vivo were observed using parental tumor cells without first enriching for a putative CSC subpopulation based on cell surface markers. One rationale for this 
approach is the lack of comprehensive information on the markers that define TICs in the PyMT model. In a similar PyMT tumor cell transplant paradigm to ours, the population of CD24 (heat stable antigen $)^{\text {hi }} / \mathrm{CD} 29\left(\beta_{1}\right.$-integrin $)+/ \operatorname{CD} 61\left(\beta_{3}\right.$-integrin $)+$ cells was found to significantly increase during tumor progression, specifically at the transition from hyperplasia to carcinoma. Greater than $90 \%$ of cells were characterized as CD $24 \%$ hi $29+/ C D 61+$ in late-stage carcinomas, and this population also had enhanced invasive potential in vitro [118]. In addition, researchers in the Visvader laboratory have shown that, in cells derived from PyMT adenomas (early-stage lesions), CD14 and c-kit, along with CD49f/CD24, enriches for cells with colony-forming potential [219]. More recently, it was shown that CD24/CD90 enriches for stem cells in the PyMT model as only the CD24+/CD90+ cells were able to form pulmonary metastases following tail vein injection as compared to the CD24/CD90 depleted cells [220]. Moreover, whereas there was no significant difference in proliferation between the populations in primary tumorigenesis, there was a large and significant increase in proliferation of the CSC population during lung colonization, suggesting it is the stem cell population that initiates lung metastasis in the PyMT model.

In addition, the physical stress of flow sorting decreases cell viability, which therefore directly influences the estimated TIC frequency as determined through limiting dilution transplantation. As observed for the MMTV-Neu model, the TIC frequency of unsorted cells was 1 in 61, decreasing to 1 in 177 for cells enriched by sorting [119]. Likewise, for cells isolated from PyMT adenomas, the TIC frequency was estimated to be 1 in 556 for unsorted cells, whereas the TIC frequency in Lin $^{\text {neg }}$ cells following sorting for CD24 was 1 in 648 [219]. Using unsorted cells isolated from late-stage carcinomas of the PyMT model, we observed a TIC frequency of 1 in 82 . It is possible that the TIC frequency in the PyMT model may vary based upon both the stage of progression and subtle differences in technical procedures among laboratories.

The specific cell surface markers that enrich for breast tumorsphere or TIC activity are also likely to vary in each mouse model. In the Balb/C $\mathrm{p} 53^{-/-}$model, mammary tumor cells double-positive for CD24 and CD29 were found to exhibit TIC activity [121]. In contrast, in the MMTV-Neu model Sca-1+ cells were found to correlate with sphere formation [221], and CD61 was also described as a marker that enriched for TICs [119]. How HIF-1 $\alpha$ directly affects various tumor cell subpopulations defined through flow cytometric profiling for the known murine mammary stem cell markers, or whether HIF-1 $\alpha$ is preferentially expressed in a given subpopulation requires further extensive investigation.

\section{Conclusion}

Tumor hypoxia profoundly affects all aspects of tumorigenesis, including tumor growth, angiogenesis, metastasis and response to chemotherapy and radiation. Although HIF's effects are pleiotropic, evidence for the role of HIF-dependent TICs in controlling these phenotypes and the solid tumor stem cell niche is increasing. Notably, a recent report indicated that oxygen tension can profoundly influence TICs because repetitive cycles of hypoxia and reoxygenation promoted breast cancer cell lines to permanently 
acquire stemlike properties [222]. Our studies demonstrate that the hypoxic response, specifically through HIF-1 $\alpha$, is important for controlling breast cancer stem cell behavior through the regulation of CD133 and the Notch pathway. These data, together with previous observations that the HIFs directly mediate glioma, lymphoma and AML TIC activity $[60,115]$, suggest that attenuation of HIF activity may effectively eradicate TICs in a variety of cancers, leading to improved therapeutic response and overall survival.

We acknowledge the possibility that HIF-1 $\alpha$ may regulate activity of TICs and differentiated cell lineages simultaneously within a tumor. For example, HIF-1 $\alpha$ may regulate breast TIC activity in a stemlike cell population expressing CD133, but HIF-1 $\alpha$ activity may also be required by differentiated cells that may act to support TICs through paracrine signaling, perhaps via a HIF-dependent secreted growth factor such as VEGF. Whether the EGFR pathway is required for HIF-1 $\alpha$-dependent regulation of TIC activity, particularly in basal-like TNBC, also requires further investigation. Given the pleiotropic role of HIF- $1 \alpha$ in tumorigenesis and metastasis, as well as the increasing evidence that stem cells may evolve de novo from non-stem cells, targeting both the TIC and non-TIC ("bulk") populations in breast cancer is likely necessary to successfully treat primary breast cancer and to prevent metastasis. 


\section{CHAPTER 4. ITGA6 (CD49F) IS DIRECTLY REGULATED BY HYPOXIA- INDUCIBLE FACTORS (HIFS) AND PROMOTES TUMOR INITIATION AND LUNG METASTASIS}

\section{Introduction}

One of the most widely used approaches to enrich for cells with stem-like features is the use of antibodies against cell surface markers followed by FACS cytometry. Several cell surface markers routinely used to enrich for CSCs were recently reported to be regulated by hypoxia in a HIF-1-dependent manner, including CD44 and CD24 (heat stable antigen) $[223,224]$. Notably, the combination of CD44 and CD24 was the first marker set used to enrich for CSCs/TICs in solid tumors, identifying a sub-population of TICs $\left(C D 44^{\text {hi }} / \mathrm{CD} 24^{\mathrm{lo}}\right)$ in breast cancers [225]. In a variety of transgenic mouse models of breast cancer, CD24 is used along with other cell surface markers to enrich for mammary cells with TIC potential $[121,220,226]$. As discussed previously, hypoxia also induces expression of Prominin-1 (CD133) [117], a marker which has been widely used to enrich for putative CSCs in a variety of solid tumors, particularly in gliomas [227].

Several integrin subunits that function as receptors for extracellular matrix (ECM) proteins also serve as common cell surface markers to enrich for normal mammary stem/progenitor cells or breast CSCs. These include integrin beta 1 (ITGB1; CD29), integrin beta 3 (ITGB3; CD61) and integrin alpha 6 (ITGA6, CD49f), as reviewed in [228]. In breast cancers, the CD49 $\mathrm{f}^{\mathrm{hi}}$ population also identifies a sub-population of breast epithelial cells with basal/myoepithelial characteristics that is thought to be the origin of luminal cancers [212, 229].

ITGA6 heterodimerizes with either ITGB1 or integrin B4 (ITGB4, CD104) to bind to laminin, an abundant component of the mammary gland ECM. In particular, the $\alpha 6 / 34$ heterodimer has been implicated as key factor in mediating breast cancer cell motility and metastasis [230]. Moreover, increased ITGA6 immunoreactivity was previously shown to correlate with reduced survival of breast cancer patients [231]. Moreover, chronic hypoxia increases the cell surface expression of CD49f in MDA-MB231 cells through a Rab11-dependent mechanism [232]. Finally, as will be presented in Chapter 5, CD49f was identified in our microarray profiling screen as being up regulated in Hifla WT cells more than 2-fold.

Since CD49f expression and the HIF transcriptional core signature are both enriched in basal-like breast tumor cells relative to luminal $(\mathrm{ER}+)$ tumors [92, 93, 229], we sought to determine if CD49f might also be a direct HIF target gene. In this Chapter, I will demonstrate that ITGA6 is a direct transcriptional target of either the HIF-1 or HIF-2 transcription factors. ITGA6 mRNA and protein expression decreases upon deletion or knockdown of Hifla alone in PyMT cells, or in response to knockdown of both HIF $1 A / H I F 2 A$ in human MDA-MB-231 cells. At least three hypoxic response elements (HREs) were identified in the human ITGA6 promoter that bound either HIF-1 or HIF-2. In the PyMT model, CD49f ${ }^{\text {hi }}$ cells enriched for TIC potential as shown by limiting 
dilution transplantation. Furthermore, enriching for CD49 $\mathrm{f}^{\text {hi }}$ cells in MDA-MB-231 cells potentiated lung metastasis in a tail vein assay. The reduced tumor-initiating and metastatic-initiating potential in the CD49 $\mathrm{f}^{\text {lo }}$ tumor cells partially recapitulates the phenotypes observed upon deletion of Hifla in the PyMT model [127]. Together, these results suggest that HIF-dependent transcriptional regulation of CD49f is partially responsible for HIF's promotion of TIC activity, ultimately resulting in enhanced breast tumor growth and metastasis.

\section{Methods}

\section{Animals}

MMTV-PyMT transgenic mice $(\mathrm{FVB} / \mathrm{Nj})$ were originally obtained from Dr Kent Hunter (National Cancer Institute). FVB/Nj and homozygous NOD.Cg-

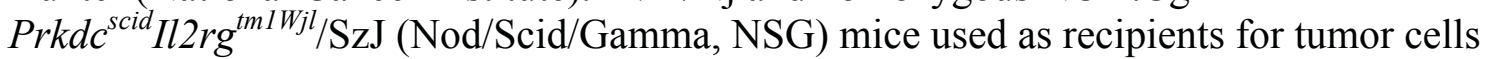
were purchased from The Jackson Laboratory (Bar Harbor, ME, USA) or bred in-house. All animal procedures were approved by the Institutional Animal Care and Use Committee at the University of Tennessee Health Science Center.

\section{Cell Culture}

PyMT WT and KO MTECs were generated and propagated in cell culture as described in Chapter 3 and [127]. MDA-MB-231 cells in which HIF1A, HIF $2 A$, or both genes, were stably knocked down by targeting with shRNAs generated as in [233] and characterized as in [1]. Parental MDA-MB-231 cells were authenticated by DDC Medical and results compared to the ATCC STR database. Cells were grown in DMEM-Hi, supplemented with 10\% FBS, 1x antimycotic-antibiotic (AA) (Sigma, St Louis, MO, USA), $25 \mathrm{mM}$ HEPES and shRNA selection antibiotics [single knockdown (KD): shHIF1A or shHIF2A cells, $4 \mu \mathrm{g} / \mathrm{ml}$ puromycin, or double knockdown (DKD): $8 \mu \mathrm{g} / \mathrm{ml}$ puromycin and $2 \mathrm{mg} / \mathrm{ml}$ hygromycin B]. Spent media from confluent cells were routinely screened for mycoplasma using the MycoAlert kit (Lonza, Basel, Switzerland).

\section{Flow Cytometry and Sorting}

Cells grown in monolayer were detached with $2.5 \%$ trypsin/EDTA, washed several times with HBSS and resuspended in flow buffer (HBSS containing 2\% FBS, 10 $\mathrm{mM}$ HEPES, and $1 \mathrm{mg} / \mathrm{mL}$ DNase I). Cell number and viability were verified by trypan blue staining prior to staining. Cells $\left(1 \times 10^{6}\right.$ in $200 \mu$ flow buffer $)$ were stained with the indicated primary antibodies (BD Biosciences, San Jose, CA) on ice for 1 hour: biotinconjugated mouse lineage panel (cat\#559971), CD31biotin (cat\#553371) CD49f-FITC (cat\#555735). Samples were rinsed once with $2 \mathrm{~mL}$ flow buffer, pelleted and kept on ice 
until analysis at the UTHSC Flow Cytometry core on a LSR II flow cytometer. All raw data exported from DIVA were analyzed using FlowJo software.

For FACS analysis of cells from digested tumors, cultured HIF-1 WT and KO PyMT MTECs were transplanted into the right inguinal mammary fat pad of 3-week old $\mathrm{FVB} / \mathrm{Nj}$ female recipients (50,000 cells input), as described in [127]. Tumors (350-750 $\mathrm{mm}^{3}$ ) minced to a fine paste and digested with collagenase type III (Worthington Biochemical Corp, Lakewood, NJ, USA) to prepare single cells for staining prior to FACS analysis. For cell sorting, $40 \times 10^{6}$ cells derived from either cultured PyMT or MDA-MB-231 cells, or digested PyMT tumors, were stained as above, rinsed with flow buffer, and sorted for purity using the $100 \mu \mathrm{m}$ nozzle on a FACSAria cytometer using the gating strategy described in [127].

After exclusion of dead cells and doublets, cells were sorted into either of two gates: for PyMT, CD $49 \mathrm{f}^{+} / \mathrm{CD} 24^{\text {high }}$ or $\mathrm{CD} 49 \mathrm{f}^{\text {neg }} / \mathrm{CD} 24^{\text {low }}$, or for MDA-MB-231 cells CD49f $\mathrm{f}^{\text {high }}$ or CD49f $\mathrm{f}^{\text {low }}$. Sorted cells were collected into $4.5 \mathrm{~mL}$ tubes pre-coated with $100 \%$ FBS and filled with $1 \mathrm{~mL}$ of DMEM $+20 \%$ FBS. Post-sort analysis was performed to verify purity and viability (7-AAD) of sorted populations. Cell viability was reconfirmed in the laboratory by trypan blue staining and hemacytometer analysis just prior to cell culture or to injection into live mice.

\section{Limiting Dilution Transplantation}

Four cell inputs $(25,50,100$ or 200 cells) per sorted cell population $\left(\mathrm{CD} 49 \mathrm{f}^{+} / \mathrm{CD} 24^{\text {hi }}\right.$ or $\left.\mathrm{CD} 49 \mathrm{f}^{\text {neg }} / \mathrm{CD} 24^{\text {low }}\right)$ were injected into the cleared mammary fat pads of 3-wk old $\mathrm{FVB} / \mathrm{N}$ recipients ( $\mathrm{n}=$ at least 8 mice/cohort) in a volume of $10 \mu \mathrm{l}$ of 1:1 HBSS: growth-factor reduced Matrigel, BD Biosciences, San Jose, CA). Mice were palpated 1-2x/week and tumors measured with digital calipers. Lesions were scored positive for tumor initiation when the diameter of the lesion was $\geq 5 \mathrm{~mm}$ in diameter. Data was input into the Extreme Limiting Dilution Analysis (ELDA) software for estimation of CSC frequency, as in [127].

\section{Tail Vein Injection of MDA-MB-231 Cells into NSG Recipients}

MDA-MD-231 cells were mock-sorted (no primary antibody), or stained with CD49f-FITC and either not sorted or sorted for CD49f $\mathrm{hi}^{\mathrm{hi}} \mathrm{vs}$. CD49f $\mathrm{f}^{\mathrm{lo}}$ sub-populations (10$20 \%$ high versus low gates). Post-sort, cells were counted by hemacytometer and adjusted to 150,000 to 185,000 cells/100 $\mu \mathrm{l}$ HBSS. Cells were injected into the pre-warmed tail vein of NSG recipient mice ( $\mathrm{n}=7 /$ cohort) and euthanized 7 weeks later. Lungs of anesthetized mice were harvested and processed for histology as in [127]. Image $\mathbf{J}$ analysis software was used to calculate the mean area of lungs occupied by metastases. 


\section{Tumorsphere Assay}

Single cells derived from digested PyMT+ tumors were FACS-sorted based on the expression of CD49f and CD24 into two populations: CD49f $\mathrm{f}^{+} / \mathrm{CD} 24^{\mathrm{hi}}$ and $\mathrm{CD} 49 \mathrm{f}^{\text {neg }} / \mathrm{CD} 24^{\text {lo }}$. A subset of sorted cells from each population was immediately plated at a density of 15 cells/ $\mathrm{ml}$ into ultra low adhesion (ULA) tissue culture plates (Corning, NY, USA) containing tumorsphere medium and tumorsphere formation efficiency (TSFE) calculated as in [127]. Data shown is representative of three independent experiments.

\section{Gene Expression}

Total RNA quality was assessed by the Agilent 2100 Bioanalyzer (Santa Clara, CA, USA) and converted to cDNA using the High-Capacity cDNA reverse Transcription kit (Applied Biosystems, Foster City, CA, USA). Optimized primer and probe sets were designed with the Universal Probe Library Assay Design Center software (Roche Applied Science, Indianapolis, IN). Ints3 (integrator complex subunit 3) or CYPA (cyclophylin A) were used as a loading control for murine or human samples, respectively. The advanced relative quantification algorithm of the Roche LightCycler 480 system was used to calculate Itga6 expression relative to the loading control.

\section{Immunofluorescence}

PyMT MTECs or MDA-MB-231 cells were plated onto tissue-cultured treated glass chamber well slides in standard culture media. At 80\% confluence, cells were placed at normoxia or hypoxia $\left(0.5 \% \mathrm{O}_{2}\right)$ for 6 additional hours. Slides were fixed with 24\% paraformaldehyde (PFA) for 10 minutes at room temperature followed by immunostaining with anti-CD49f-FITC (BD Biosciences) or anti-CD49f (Chemicon) followed by anti-rat IgG Alexa Fluor ${ }^{\circledR} 488$. For tumorspheres, endpoint spheres were dried onto glass slides for 15 minutes at $37^{\circ} \mathrm{C}$, post-fixed with $4 \%$ PFA for 15 minutes and immunostained with CD49f-FITC.

\section{Promoter Analysis and Chromatin Immunoprecipitation (ChIP)}

The promoter of ITGA6 was scanned for the presence of putative HIF response elements (HREs) using Transcription Factor Matrix (TFM) Explorer. A $2500 \mathrm{bp}$ sequence $(-2000$ to +500$)$ was scanned for the consensus HRE sequence using weight matrices available from JASPER and TRANSFAC. Primers were designed to three independent HREs in the human ITGA6 promoter. MDA-MB-231 shEmpty (empty pLKO. 1 vector) or shHIF1A transduced cells were cultured at $21 \%$ (normoxia) or $0.5 \%$ $\mathrm{O}_{2}$ (hypoxia) for 6-24 hours, and ChIP performed using primary antibodies against Histone H3 (AbCam 1791), HIF-1 $\alpha$ (AbCam 2185 or Santa Cruz sc-10790X), HIF-2 $\alpha$ (Novus NB100-122), or rabbit IgG, as the non-specific control. 


\section{Breast Tumor Subtype Analysis}

ITGA6 mRNA expression across breast cancer subtypes was analyzed using two independent data sets. The Cancer Genome Atlas [93] data was obtained from http://tcgadata.nci.nih.gov/tcga/tcgaHome2.jsp. The GSE1992 [234] data set was downloaded from the Gene Expression Omnibus website (GEO, http://ncbi.nlm.nih.gov/projects/geo). Boxplots depicting ITGA6 mRNA expression stratified by PAM50 classification were generated in Prism, and $p$-values calculated following ANOVA analysis with Bonferroni correction.

\section{Kaplan-Meier Curves}

Survival analysis data was derived from the GSE1992 microarray data set. Normalized ITGA6 expression values were divided into four equal quartiles based on distribution frequencies. Forty-nine tumors were identified in the top quartile ( $\geq 75 \%$ percentile) with "high" ITGA6 expression ( $\geq 0.484)$, and 49 tumors were identified in the lowest quartile ( $\leq 25 \%$ percentile) with "low" ITGA6 expression $(\leq-0.813)$. Survival analyses were performed in Prism and $p$-values derived using the log-rank test.

\section{Results}

\section{Deletion of HIF1A Down-Regulates CD49f Expression}

To assess if HIF-1 regulates expression of CD49f, Hifla WT and KO MTECs derived late stage PyMT carcinomas were grown at normoxia or hypoxia and CD49f levels evaluated by quantitative real-time PCR (qRT-PCR) and immunostaining. Expression of Itga6 mRNA increased 2-fold in WT cells in response to hypoxia (6h), and this hypoxic induction was reduced in HIF-1 KO cells. At normoxia, expression of Itga6 mRNA was decreased by 50\% in KO cells (Figure 4-1A). Similarly, examination of CD49f protein levels by immunofluorescence (IF) staining of WT and KO cells revealed a decrease in CD49f signal in normoxic KO cells relative to WT cells; this effect was more striking at hypoxia (Figure 4-1B). However, FACS cytometry profiling using the same antibody as used in IF (CD49f-FITC) did not reveal any significant changes in the mean fluorescence intensity of CD49f between WT and KO PyMT cells.

To determine whether CD49f is differentially expressed in WT and KO tumors in vivo, WT and KO MTECs were implanted into syngeneic FVB hosts to generate tumors. Single cells from digested tumors were stained with CD49f-FITC and subjected to FACS profiling ( $\mathrm{n} \geq 5$ tumors/genotype). As compared to the isotype control, the mean enrichment in CD49f MFI was 1.8 fold higher in WT versus KO tumors (Figure 4-1C). When total RNA from these same tumors was analyzed by RT-PCR, the mean expression level of Itga6 mRNA was decreased $\sim 2$-fold in KO tumors. Although this effect was small, it was statistically significant and consistent with data from cultured cells 
Figure 4-1. ITGA6 (CD49f) is down-regulated upon deletion of Hifla in PyMT cells, tumors and in HIF-1 $\alpha$ KO tumorspheres.

A. HIF-1 $\alpha$ WT or KO cultured cells were analyzed for relative Itga6 mRNA levels at normoxia or following 6 hours hypoxia $(* * p<0.01, * * * p<0.001$, ANOVA with Bonferroni post-test, $\mathrm{n}=3$ biological replicates/genotype, representative of one experiment). Fold change is relative to KO mRNA levels at normoxia which was set to 1.0. B. WT and KO cultured cells were exposed to normoxia or 6 hours hypoxia, then immunostained for CD49f-FITC and counterstained with DAPI. Images were captured at 200x magnification; the scale bar represents $20 \mu \mathrm{m}$. C. Representative CD49f FACS staining histograms of WT (black histogram) and KO (blue histogram) tumors subjected to FACS analysis. The isotype only antibody control is also plotted (red histogram). Data is representative of $n \geq 5$ tumors/genotype. The percentage of tumor cells that were CD49f positive was determined based on the live, singlet, Lin $^{\text {neg }}$ parent population using FlowJo. D. Average fold change CD49f mean fluorescence intensity (MFI) between replicate FACS analyses ( $p<0.01$, Student's $t$-test, $\mathrm{n} \geq 5$ tumors/genotype) E. Average fold change in Itga6 mRNA in HIF-1 $\alpha$ WT and KO tumors, each relative to control $(p<0.01$, Student's $t$-test). F. End-point HIF-1 $\alpha$ WT and KO tumorspheres were dried onto glass slides, stained with CD49f-FITC, counterstained with DAPI and imaged by confocal microscopy. Scale bar represents $50 \mu \mathrm{m}$. 

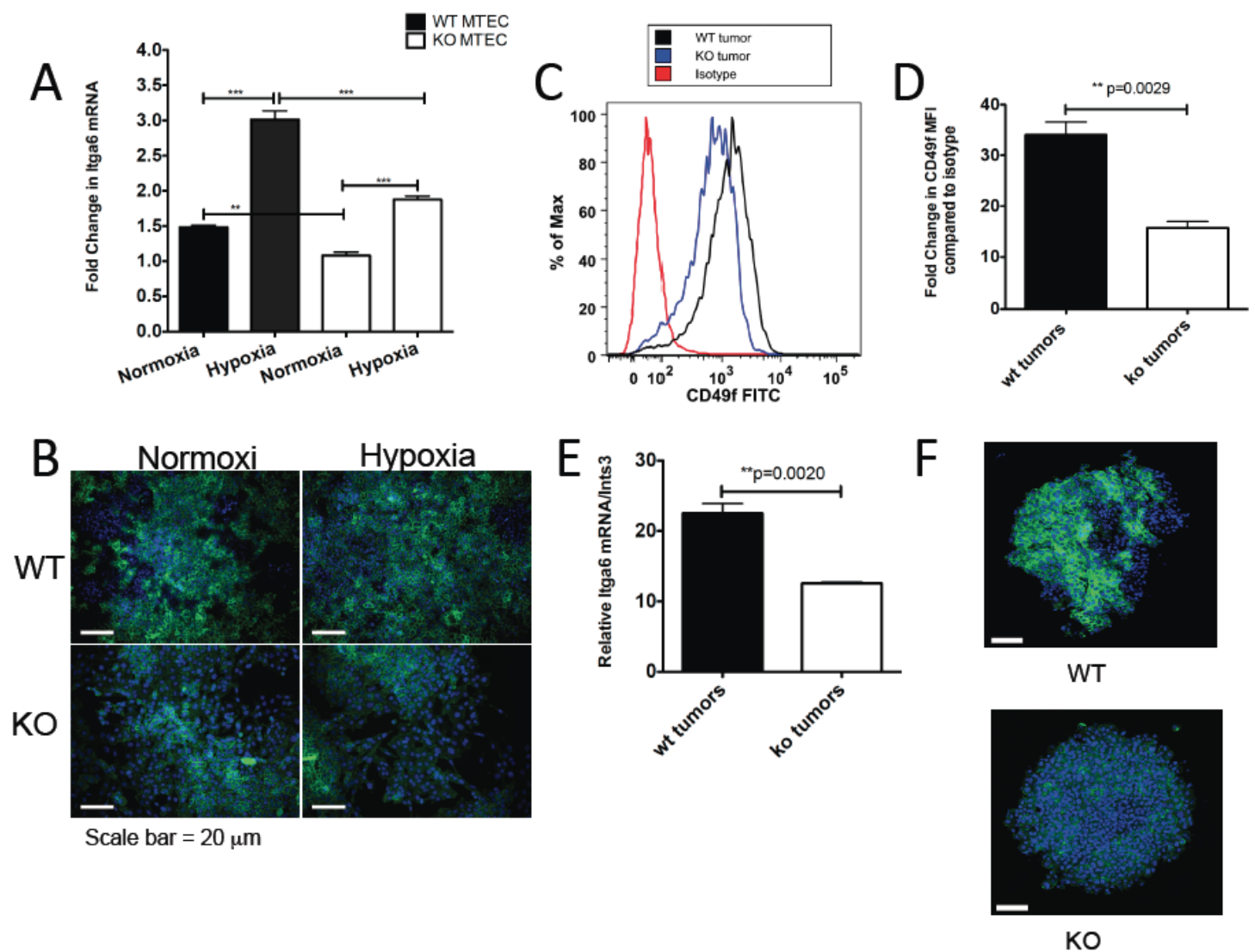
(Figure 4-1E). Together, these results suggest that hypoxia/HIF-1 regulate CD49f at both the mRNA and protein levels.

Previously we showed that deletion of HIF-1 significantly decreased tumorsphere formation efficiency (TFE) in the PyMT model and reduced expression of CD133 in tumorspheres [127]. Tumorspheres derived from KO MTECs, also exhibit reduced expression of CD49f as compared to WT tumorspheres (Figure 4-1F).

\section{Deletion of HIF1A Alone or Together with $H I F 2 A$, Results in Decreased Expression of CD49f by the Human Breast Cancer Cell Line MDA-MB-231}

To confirm that the effect of HIF1A deletion on CD49f was not restricted to the PyMT model, we analyzed the effect of HIF $\alpha$ deletion on CD49f expression in the human breast cancer cell line MDA-MB-231. MDA-MB-231 cells were previously shown to be CD49f $\mathrm{f}^{+} / \mathrm{CD} 24^{\mathrm{Neg}}$ [229]. CD49f expression was profiled in these cells (Figure 4-2A) in 5 independent experiments by flow cytometry. Knockdown of HIF $1 \mathrm{~A}$ alone decreased CD49f by a mean of 1.58 -fold, which increased to 2.27 fold in DKD. Knock down of HIF2A alone did not reduce CD49f expression (Figure 4-2B).

Immunofluorescence staining of MDA-MB-231 cells cultured on coverslips revealed a modest hypoxic induction of CD49f. While there was a slight decrease in CD49f signal intensity in the shHIF1A cells as compared to EV or shHIF2A cells, the combination of both HIF $1 A$ and HIF $2 A$ knockdown resulted in almost no CD49f expression (Figure 4-2C). These data suggest that CD49f expression is regulated by the HIF transcription factors, but is more sensitive to HIF- $1 \alpha$ than HIF- $2 \alpha$ loss. Of note, IF staining is still being optimized to repeat and verify these results.

To determine if the HIFs regulate expression of CD49f, we cultured all four MDA-MB-231 genotypes at normoxia and hypoxia and analyzed the expression of ITGA6 mRNA. Hypoxia did not increase baseline mRNA levels of ITGA6 in control cells (shEmpty). Expression of ITGA6 mRNA was $~ 50 \%$ lower in shHIF1A cells as compared to shEmpty cells at normoxia or hypoxia. In contrast, decreased ITGA6 mRNA relative to shEmpty cells was only observed at 6 or 24 hours of hypoxia. Independent of oxygen concentration, there was a $>3$-fold decrease in ITGA6 expression when HIFIA and $H I F 2 A$ were simultaneously knocked down (Figure 4-2D). In agreement with results from cultured cells, mammary gland tumors derived from all four MDA-MB-231 genotypes transplanted into NSG mice also showed decreased expression of ITGA6 mRNA upon knock down of $H I F 1 A$ alone or in conjunction with $H I F 2 A$. There was no change in ITGA6 mRNA in tumors derived from shHIF2A cells compared to shEmpty tumors (Figure 4-2E). 

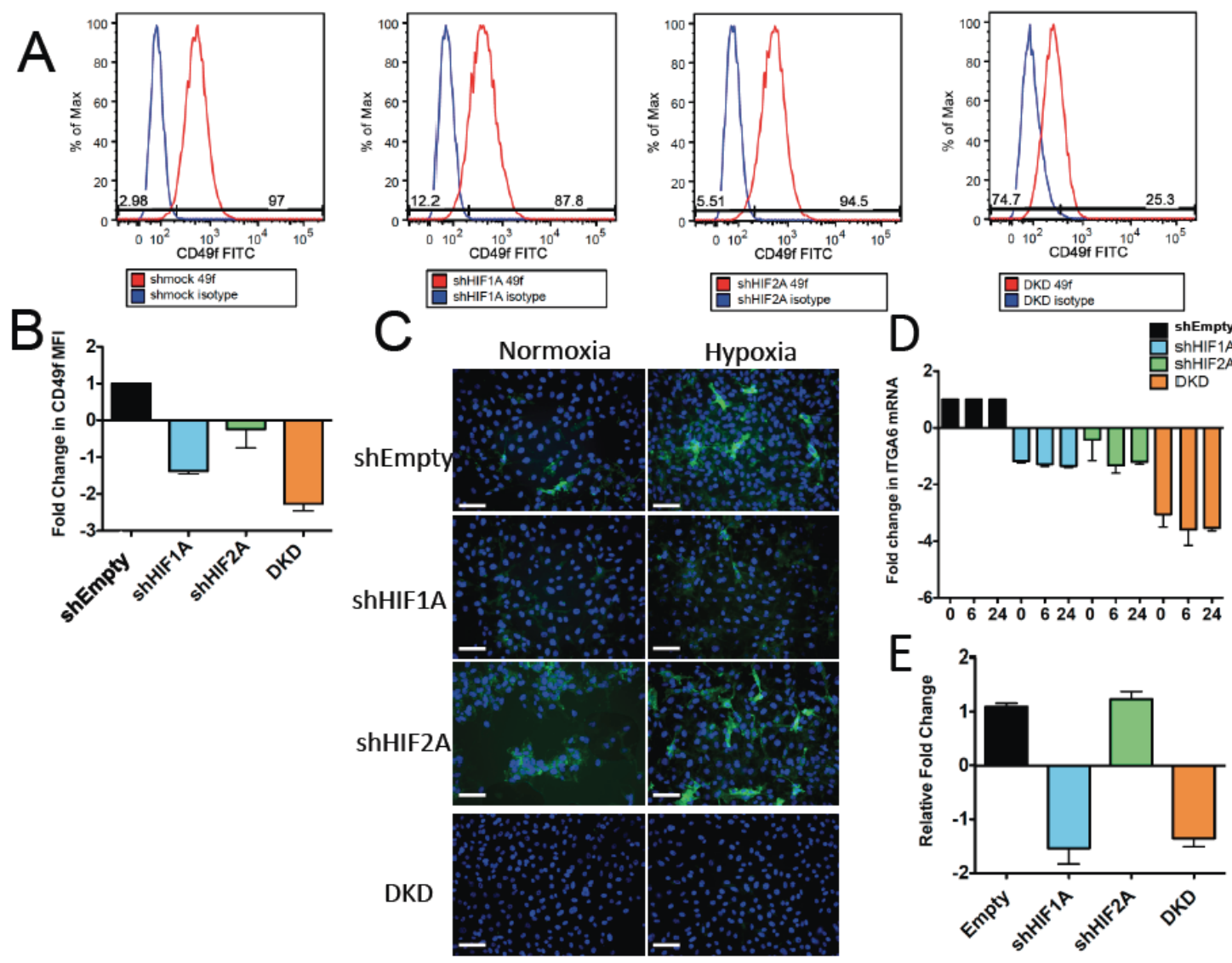

Figure 4-2. CD49f expression is reduced in $H I F 1 A$ and $H I F 2 A$ double knockdown MDA-MB-231 cells and tumors.

A. MDA-MB-231 EV, shHIF-1 $\alpha$, shHIF-2 $\alpha$ and DKD cultured cells were stained for CD49f-FITC and subjected to FACS analysis. Plots represent the maximum fluorescent intensity of stained cells (red) versus the isotype control (blue, representative of 4 individual experiments). B. Multiple cytometry analyses were combined to determine the average fold change in mean fluorescence intensity (MFI) among the four genotypes $(n=4$ independent experiments). C. Cells were plated on tissue culture glass slides and cultured until $90 \%$ confluent prior to being subjected to normoxia or hypoxia for 6 hours. Cells were fixed with 4\% PFA and stained for CD49f. Scale bar represents $20 \mu \mathrm{m}$. D-E. Mean fold change in ITGA6 mRNA expression in cultured MDA-MB-231 cells ( $\mathrm{n}=3 /$ genotype) and end-stage tumors ( $\mathrm{n}=3$ tumors/genotype). mRNA expression was evaluated after 6 and 24 hours of hypoxic exposure. shEmpty at 24 hours normoxia ( 0 hours hypoxia) was set to represent a fold change of 1 . 


\section{ITGA6 (CD49f) Is a Direct HIF Transcriptional Target Gene}

Due to the apparent HIF $\alpha$-dependent regulation of ITGA6 mRNA we observed in both PyMT and MDA-MB-231 cells, we hypothesized that ITGA6/CD49f was a direct HIF target. Upon examination of the ITGA6 promoter, three potential HIF Response Elements (HRE) were identified upstream of the transcriptional start site (TSS) in human ITGA6 (Figure 4-3A). The three sites were located at $-170,-1333$, and -1762 base pairs from the TSS. One potential conserved HRE site was identified in the mouse Itga6 promoter at -1690 from the TSS. To examine HIF-1 $\alpha$ recruitment to the CD49f promoter, we performed chromatin-immunoprecipitation (ChIP) assays using MDA-MB-231 shEmpty (EV) and shHIF1A cells cultured at hypoxia for 6 hours. HIF-1 $\alpha$ was strongly recruited to both the -170 and $-1762 \mathrm{HRE}$ sites in EV cells relative to shHIF1A cells (Figure 4-3B, upper panel). In contrast, there was very weak enrichment of HIF-1 $\alpha$ bound to the -1333 site. A previously characterized HRE site in the HIF- $1 \alpha$ target gene erythropoietin (EPO) was used a positive control for HIF-1 $\alpha$ binding (Figure 4-3), and histone $\mathrm{H} 3$ as a general active transcription control. To determine if HIF- $2 \alpha$ could compensate for loss of HIF-1 $\alpha$ during CD49f transcription, ChIP assays were repeated using an anti-HIF-2 $\alpha$ antibody. The same putative HRE sites were analyzed by qRTPCR. At both the -170 and -1762 sites, HIF-2 $\alpha$ was weakly bound in WT cells (Figure 4-3B, lower panel). However, in the shHIF1A cells, HIF-2 $\alpha$ binding increased, suggesting a compensatory role for CD49f regulation with loss of HIF-1 $\alpha$. These data are consistent with our prior observations that loss of HIF-1 $\alpha$ in MDA-MB-231 cells results in up-regulation of HIF-2 $\alpha$ protein [1].

\section{CD49f Promotes Primary Tumor Formation and Increases TIC Frequency in the PyMT Model}

Individual tumors harvested from a PyMT transgenic female mouse were combined and digested to obtain a single cell preparation that was used to sort MTECs based on expression of CD49f in combination with CD24. Sorted cells were injected into mice in a limiting dilution transplantation assay or plated for a tumorsphere formation assay. The tumorsphere formation efficiency (TSFE) of CD24 ${ }^{\text {high }} / \mathrm{CD} 49 \mathrm{f}^{+}$cells was 3.5 times higher than the $\mathrm{CD} 24^{\text {low }} / \mathrm{CD} 49 \mathrm{f}^{\text {neg }}$ cells (representative of $\mathrm{n}=2$ biological replicates, $p=0.0001$ ) (Figure 4-4A). When transplanted into FVB hosts, CD24 $4^{\text {high }} / \mathrm{CD} 49 \mathrm{f}^{+}$MTECs resulted in primary tumors that were larger in volume at study endpoint than tumors derived from $\mathrm{CD} 24^{\text {low }} / \mathrm{CD} 49 \mathrm{f}^{\text {neg }}$ MTECs (Figure 4-4B). In addition, when analyzed by ELDA, CD24 $4^{\text {high }} / \mathrm{CD}_{4} 4 \mathrm{f}^{+}$derived tumors showed a significantly higher frequency for TICs than the CD $24^{\text {low }} / \mathrm{CD} 49 \mathrm{f}^{\text {neg }}$ derived tumors ( 1 of 99 vs. 1 of 578 , respectively, $p=0.00043$ ) (Table 4-1). These data indicate that MTECs with high expression of CD24/CD49f have a higher tumor initiating potential than CD24 low $/$ CD $49 f$ cells.

At experiment endpoint, primary tumors derived from the transplanted sorted cells were harvested, digested to a single cell preparation and analyzed by flow cytometry (Figure 4-4C). Tumors derived from CD2 $4^{\text {high }} / \mathrm{CD} 49 \mathrm{f}^{+}$MTEC were composed of 2 distinct cell populations: CD24 $4^{\text {high }} / \mathrm{CD} 49 \mathrm{f}^{+} \mathrm{MTECs}$ and CD $24^{\text {low }} / \mathrm{CD} 49 \mathrm{f}^{\text {neg }} \mathrm{MTECs}$, 

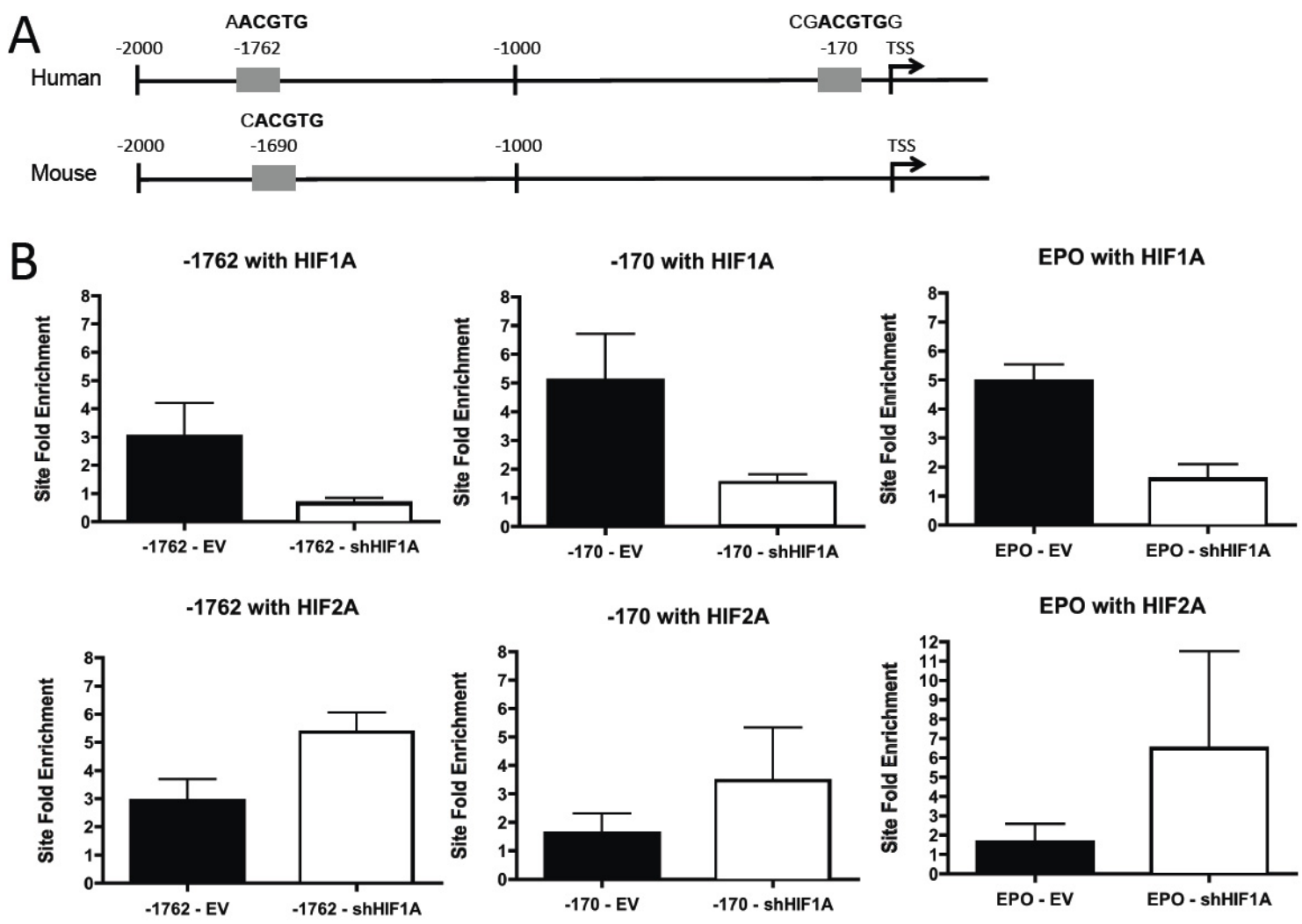

Figure 4-3. ITGA6 (CD49f) is a direct HIF target gene in MDA-MB-231 cells. A. A schematic representation of HREs in the proximal promoter of ITGA6 that were assessed for HIF- $1 \alpha$ and HIF- $2 \alpha$ recruitment. B. MDA-MB-231 EV and shHIF- $1 \alpha$ cells were cultured at hypoxia $\left(0.5 \% \mathrm{O}_{2}\right)$ for 6 hours. ChIP assays were performed with HIF$1 \alpha$ (upper panels) and HIF-2 $\alpha$ (lower panels) and qRT-PCR conducted on the isolated DNA to determine HIF $\alpha$ recruitment. ChIP for the EPO promoter was conducted as a positive control. Binding enrichment for each antibody (site fold enrichment) represent antibody binding at each HRE site over IgG control. Each panel represents the combination of replicate biological experiments ( $n=3$ for HIF- $1 \alpha$ and $n=2$ for HIF- $2 \alpha$ for each comparison). 

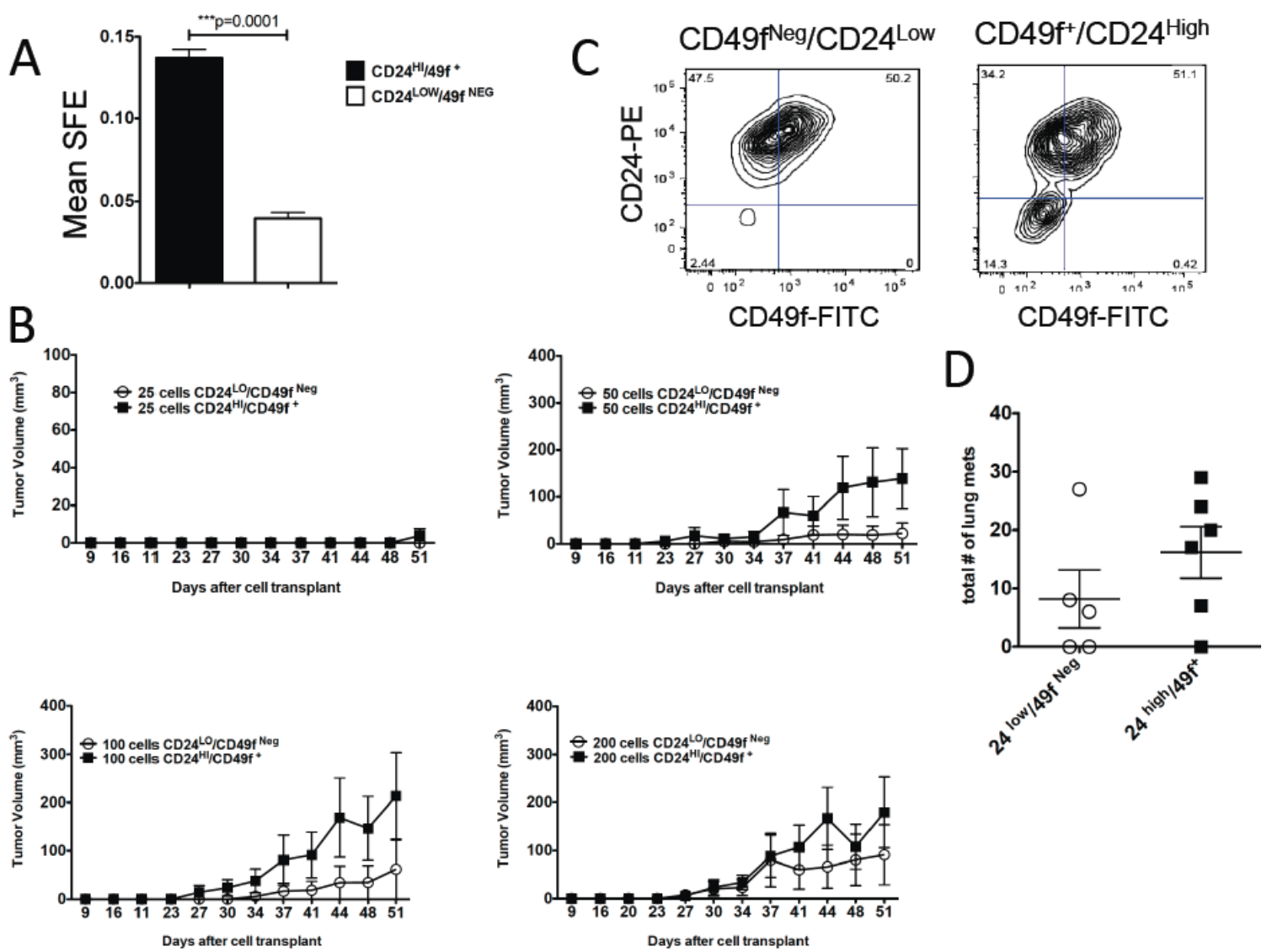

Figure 4-4. PyMT CD24 ${ }^{\text {hi }}$ CD49f $^{+}$cells are enriched for TIC activity in vitro and in vivo.

A. PyMT transgenic tumors were harvest, digested, and stained with CD49f and CD24 antibodies prior to being subjected to FACS analysis. CD $24{ }^{\text {hi }} / \mathrm{CD} 49 \mathrm{f}^{+}$(double positive, $\mathrm{DP}$ ) and $\mathrm{CD} 24^{\text {low }} / \mathrm{CD} 49 \mathrm{f}^{\text {neg }}$ double negative (DN) cells were collected and plated for tumorsphere formation efficiency (A) or (B) used in a limiting dilution transplantation assay. Mean SFE/well \pm S.E.M. Cells were plated at a density of 30,000 cells/well in 6well format and mean SFE/well \pm S.E.M. determined ( $\mathrm{n} \geq 8$ wells/genotype, unpaired Student's $t$-test). B. Comparison of DP and DN mean tumor volume $\pm \mathrm{SEM}$ over time when $25,50,100$ or 200 cells are injected into the mammary fat pad. C. CD49f/CD24 expression was analyzed in tumors derived from initial transplantation of DP (right panel) and DN (left panel). D. DP and DN recipient mice ( $\mathrm{n}=5 /$ genotype) were evaluated for lung metastases at time of primary tumor removal. Graph represents mean number of metastases \pm S.E.M. While the data trended toward significance, there were not enough animals within each cohort to power the study to detect smaller changes in metastatic potential. 
Table 4-1. ELDA analysis of TIC frequency in CD24 $4^{\text {high }} /$ CD49f $\mathrm{f}^{+}$or CD24 ${ }^{\text {low }} / \mathrm{CD} 4 \mathrm{f}^{\text {neg }}$ derived tumors at day 51 post transplant.

\begin{tabular}{lccc}
\hline \multirow{2}{*}{ Number of Cells Injected } & \multicolumn{2}{c}{ MTEC Phenotype } & \\
\cline { 2 - 3 } & $\mathbf{C D 2 4}^{\text {Low }} /$ CD49f $^{\text {Neg }}$ & C24 $^{\text {High }} /$ CD49f $^{+}$ & $\boldsymbol{p}_{\text {-Value }}$ \\
\hline 200 & $2 / 7(29 \%)$ & $5 / 7(71 \%)$ & \\
100 & $1 / 6(17 \%)$ & $4 / 6(67 \%)$ & \\
50 & $1 / 8(12 \%)$ & $6 / 9(67 \%)$ & \\
25 & $0 / 8(0 \%)$ & $1 / 8(12 \%)$ & \\
Estimated TIC Frequency & $1 / 578$ & $1 / 99$ & 0.00043 \\
\hline
\end{tabular}

suggesting that $\mathrm{CD} 24^{\text {high }} / \mathrm{CD} 49 \mathrm{f}^{+}$MTEC are able to regenerate not only the same cell population from which they were derived, but also the weakly tumorigenic $\mathrm{CD} 24^{\text {neg }} / \mathrm{CD} 49 \mathrm{f}^{\text {neg }}$ MTECs. When lungs from mice bearing either CD24 ${ }^{\text {high }} / \mathrm{CD} 49 \mathrm{f}^{+}$ MTEC or CD24 ${ }^{\text {low }} / \mathrm{CD} 49 \mathrm{f}^{\text {neg }}$ MTEC derived tumors were scored for number of metastatic lesions, mice transplanted with CD24 ${ }^{\text {high }} / \mathrm{CD} 49 \mathrm{f}^{+}$MTECs had on average $50 \%$ more total lung metastases than mice transplanted with CD $24^{\text {low }} / \mathrm{CD} 49 \mathrm{f}^{\text {neg }} \mathrm{MTEC}$ (Figure 4-4D). These data suggest that CD49f not only plays a role in primary tumor initiation, but also promotes metastatic potential from the primary mammary tumor site to the lung.

\section{CD49f Promotes Lungs Metastases Induced by Tail Vein Injection of MDA-MB-231 Cells}

To further investigate if metastatic potential is driven by CD49f, MDA-MB-231 shEmpty cells were FACS sorted based on CD49f expression levels into CD49f $\mathrm{f}^{\text {high }}$ and CD49f ${ }^{\text {low }}$ populations. When CD49 $\mathrm{f}^{\text {high }}$ cells were injected into the tail vein of NSG hosts, the area of the lungs occupied by metastases was $25 \%$ higher than the metastatic area induced by CD $49 \mathrm{f}^{\text {low }}$ cells $(p=0.001)$, and $>50 \%$ higher than 'mock sorted' (unstained cells subjected to the mechanical stress of passing through the cell sorting nozzle, $p=0.0001$ ) (Figure 4-5A-B). These data suggest that the presence of CD49f enriches for a cell phenotype with an elevated ability to colonize the lungs. Since this was only a single experiment, we aimed to replicate these results by repeating the FACS sorting and tail vein study. However, in contrast to the initial experiment, in the second experiment we stained all cells with CD49f-FITC. Cells were then sorted based on CD49h ${ }^{\text {high }}, \mathrm{CD} 49 \mathrm{f}^{\text {low }}$ or mock sorted (subjected to sorting without selecting for a subpopulation). Cells were again injected into the tail vein of NSG hosts and metastatic area of the lung analyzed after 7 weeks. In contrast to the first experiment, in which the mock sorted lungs produced very few metastases compared to either the CD49 $\mathrm{f}^{\text {high }}$ or CD49fl ${ }^{\text {ow }}$ sorted samples, this time the lung of mock sorted injected cell lungs produced a range of metastases (Figure 4-5C-D), with the majority of lungs filled at least 50\% with metastases. This result is more consistent with our prior observations that by flow cytometry $96-98 \%$ of the MDA-MB-231 shEmpty cells are positive for CD49f. It would 
A

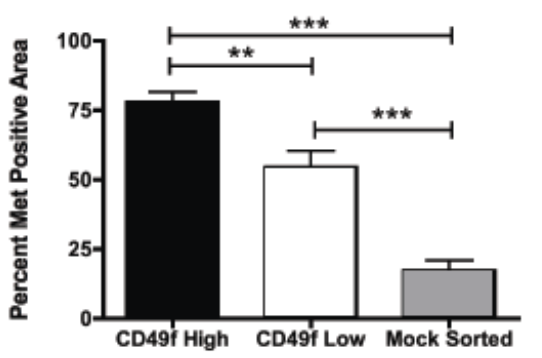

C

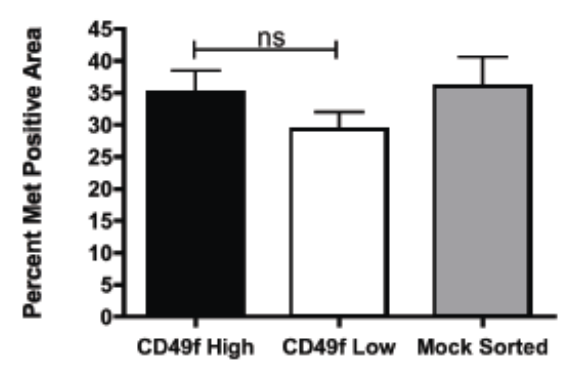

B

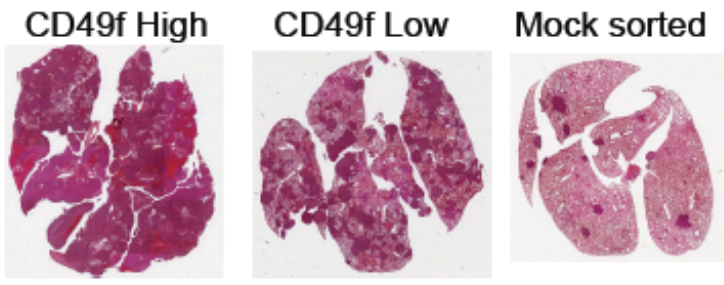

D CD49f High

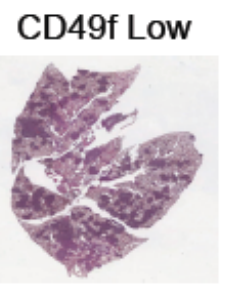

Mock sorted
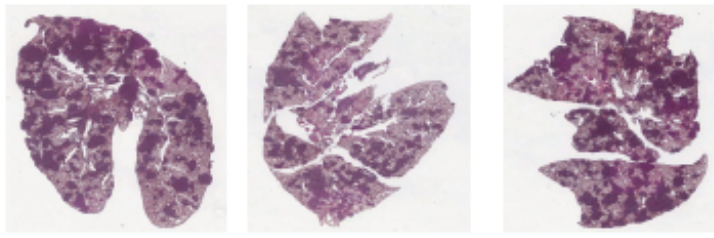

Figure 4-5. FACS-sorted CD49 $\mathrm{f}^{\text {high }}$ cells exhibit increased lung metastatic potential in a tail vein assay.

MDA-MB-231 EV cells were stained with CD49f-FITC antibody and subjected to FACS sorting. Cells collected for CD49 $\mathrm{f}^{\text {high }}$ were gated as the top $20 \%$ of CD49f expressing cells, and cells collected for CD49 $\mathrm{f}^{\text {low }}$ were gated as the lower $20 \%$. Mock sorted cells not exposed to antibody but subjected to sorting serve as the non-enriched control. Cells $(185,000$ cells $/ 100 \mu \mathrm{L})$ for each genotype were injected into the tail vein of NSG mice and lungs were harvested for NBF fixation and H\&E staining 7.5 weeks later. A. Graph of mean area of lung occupied by metastases per population $(* * p<0.01, * * * p<0.001$, ANOVA with Bonferroni post-test). B. Representative H\&E stained images of lungs from each genotype ( $\mathrm{n}=5$ lungs/cell population). The experiment was repeated, this time collecting for $\mathrm{CD} 49 \mathrm{f}^{\text {high }}$ by gating for the top $10 \%$ of CD49f expressing cells, and collecting for $\mathrm{CD} 49 \mathrm{f}^{\text {low }}$ by gating for the lower $10 \%$ of CD49f expressing cells. Mock sorted cells were stained with antibody and subjected to sorting without gating for CD49f expression. C. Graph of the mean area of lung occupied by metastases per population. B. Representative H\&E stained images of lungs from each genotype. 
be expected that injecting the mock cells, which contain a mixture of high- and lowexpressing CD49f cells would give rise to a wide range of lung metastases, with some lungs having more metastases compared to others. These data also suggest that the CD49f antibody (clone $\mathrm{GoH} 3$ ) may activate integrin signaling to stimulate metastasis.

\section{CD49f Expression Is Enriched in Specific Subtypes of Human Breast Cancers and Correlates with Decreased Survival}

Breast cancer has been classified into five major subtypes on the basis of global gene expression [86]. We sought to determine the expression of the HIF regulated TIC marker, CD49f in human breast cancers. By examining previously published microarray analyses of primary breast tumor sample [236] [234], we found that CD49f is significantly upregulated in basal-like breast cancers compared to HER2 enriched or luminal breast cancers (Figure 4-6A). As expected, increased levels of ITGA6 correlated with increased levels of $H I F 1 A$ across all subtypes. However, there was no correlation between HIF2A and ITGA6 expression (Figure 4-6B-C). We also discovered that ITGA6 expression is prognostic for survival in patients with breast cancer. Patients with tumors with high ITGA6 levels have significantly reduced overall and recurrence free survival (Figure 4-6D).

\section{Discussion}

After examining the HIF-dependent expression of several cell surface markers in the PyMT model that were previously reported to enrich for CSC activity in various transgenic models of breast cancer (CD24, CD29, CD133, CD49f), we identified CD49f as a HIF-1 $\alpha$ regulated CSC marker in PyMT tumors and in MDA-MB-231 cells. We demonstrated that upon deletion of Hifla in the PyMT murine model of breast cancer, the expression level of CD49f mRNA and protein decreases. This HIF-1 $\alpha$ effect on CD49f was observed not only in vitro, but also in vivo since mammary gland tumors derived from Hifla KO MTECs also expressed less CD49f than WT tumors. We validated these findings in the commonly used human metastatic breast cancer cell line, MDA-MB-231. $\mathrm{HIF} \alpha$ knockdown in these cells revealed that knockdown of HIF1A had a more prominent effect on CD49f than knockdown of HIF2A, while simultaneous knockdown of both HIF $\alpha$ subunits has the most significant impact on CD49f expression, decreasing expression by $>75 \%$.

Additionally, we identified 3 putative HRE sites within the human ITGA6 proximal promoter, and demonstrated that HIF-1 $\alpha$ directly binds to at least 2 of these sites. The use of the HIF1A knockdown (KD) cells in this experiment provides compelling evidence that HIF- $1 \alpha$ is direct transcriptional regulator of ITGA6, since little to no HIF-1 binding was observed in the HIF $1 A$ KD cells. Additionally, in terms of HIF$2 \alpha$ binding, there was only moderate enrichment observed at these HRE sites in the EV cells, while in the HIF IA KD cells there was a strong enrichment. These data suggest that while HIF- $1 \alpha$ may be the main regulator of ITGA6, upon HIF- $1 \alpha$ loss, HIF- $2 \alpha$ can play a 

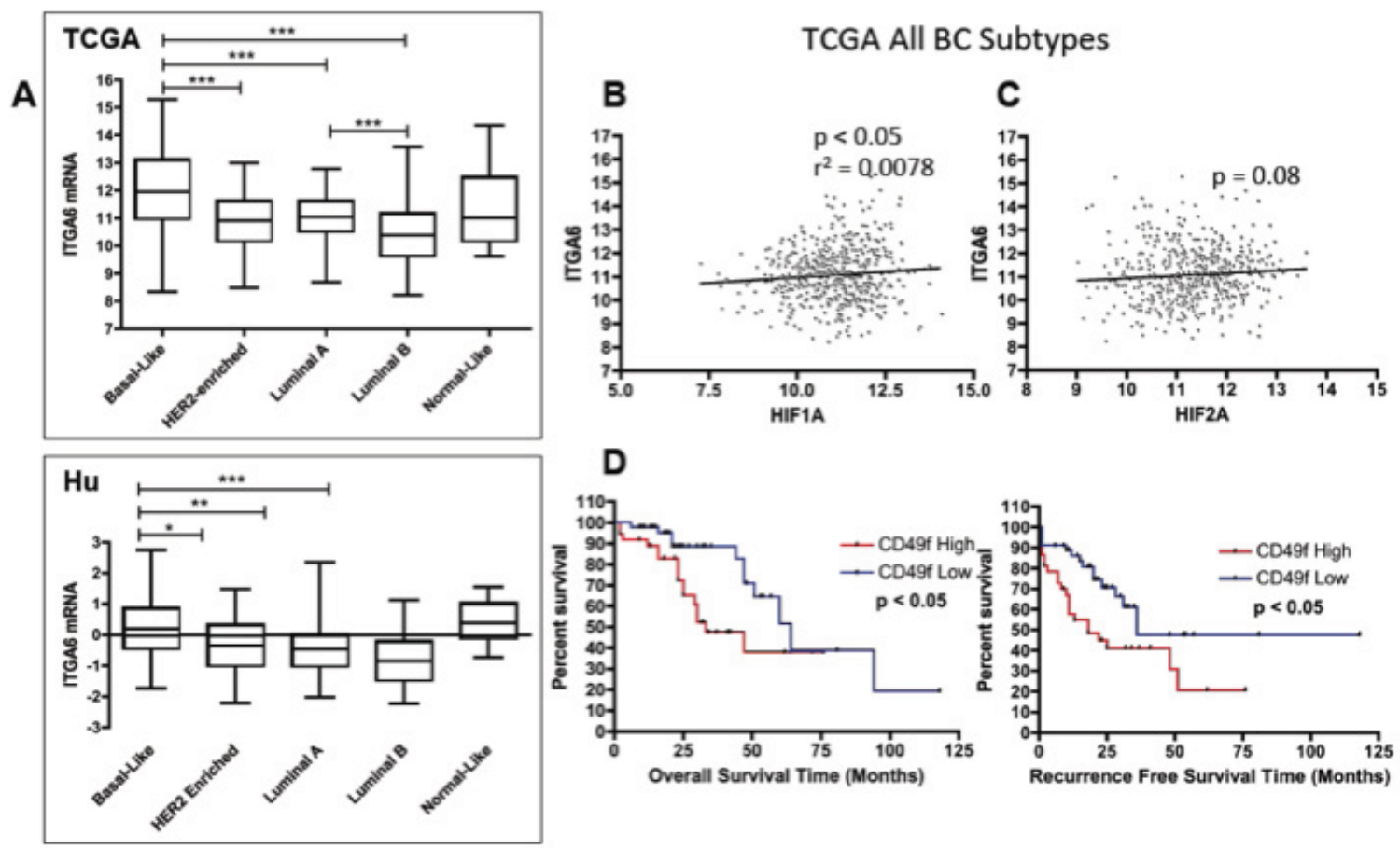

Figure 4-6. Correlation between ITGA6 and HIF1A expression and survival in breast cancer patients.

A. ITGA6 mRNA is differentially expressed in human breast cancer subtypes. Publically available datasets [93, 234] were used to compare levels of ITGA6 mRNA in breast cancer samples classified by PAM50 $\left({ }^{*} p<0.05, * * p<0.01, * * * p<0.001\right.$, ANOVA with Bonferroni post-test). B. In the TCGA dataset, ITGA6 mRNA positively correlates with HIF1A $\left(p<0.05\right.$, correlation coefficient $\left.r^{2}=0.0078\right)$ mRNA but not HIF $2 A$ mRNA $(p=$ $0.08)(C)$. D.Kaplan-Meier curves depict the probability of breast cancer survival and recurrence based on relative ITGA6 mRNA expression in the Hu data set [234] ( $\mathrm{n}=75$ tumors for high CD49f and $\mathrm{n}=75$ tumors for low CD49f, Log rank test, $p<0.05$ ). 
compensatory role and also induce CD49f transcription. These conclusions are in agreement with qRT-PCR, FACS, and immunofluorescence experiments, which revealed little to no CD49f expression or activity in DKD cells. ChIP experiments are currently in progress between shEmpty and DKD cells to further validate these data.

Upon further investigation into the role CD49f plays in primary mammary tumor formation and lung colonization, we discovered that PyMT MTECs enriched for high levels of CD49f $\left(\mathrm{CD}^{4} 9^{+}\right)$by FACS analysis had significantly increased tumorsphere formation in vitro and had enriched TIC potential in vivo as compared to cells not enriched for CD 49f $\left(\mathrm{CD} 24^{\text {low }} / \mathrm{CD} 49^{\mathrm{Neg}}\right)$. These data revealed that CD24 $4^{\text {high }} / \mathrm{CD} 49^{+} \mathrm{PyMT}$ cells are enriched $>5$-fold for TIC frequency than $\mathrm{CD}_{4} 9 \mathrm{f}^{\mathrm{Neg}}$ cells. Additionally, analysis of the metastatic potential of PyMT CD24/CD49f enriched cells revealed that the average number of metastatic lesions present in the lungs was higher in the mice transplanted with $\mathrm{CD} 24^{\text {high }} / \mathrm{CD} 49 \mathrm{f}^{+}$cells than in the mice that received CD $24^{\text {low }} / \mathrm{CD} 49 \mathrm{f}^{\mathrm{Neg}}$ cells. However, the variability of the metastases among cohorts and the small sample size did not provide enough power for sufficient statistical analyses for this experiment. It should also be noted, that after this experiment was completed, Bryan Welm shared unpublished data that he found that only CD24 ${ }^{\text {high }}$ PyMT tumor cells retain TIC activity. Therefore it is possible that in these experiments, the absence of CD2 $4{ }^{\text {high }}$ cells in the CD $49 \mathrm{f}^{\text {neg }}$ population may be confounding the results that CD49f enriches for TICs. Therefore, repeating the experiment with $\mathrm{CD} 24^{\text {high }} / \mathrm{CD} 49^{+}$and $\mathrm{CD} 24^{\text {high }} / \mathrm{CD} 49 \mathrm{f}^{\text {neg }}$, would provide further insight into the exact role of CD49f in promoting tumor growth and metastasis.

However, using the MDA-MB-231 cell line, we found that high CD49f expression augments lung metastases in the tail vein assay when MDA-MB-231 cells enriched for CD $49^{\text {High }}$ and CD49f ${ }^{\text {Low }}$ populations are compared. The metastatic lesions in the lungs of mice injected with CD49 $\mathrm{f}^{\mathrm{High}}$ cells were so numerous and large that it was not possible to discern the number of individual foci. Instead, the total area of metastatic burder lung was determined by ImageJ analysis, revealing a 50\% increase in total lung area occupied by metastases in mice that received CD $49^{\text {High }}$ cells as compared to those that received CD $49^{\text {Low }}$ cells. In agreement with our data, when 4T1 breast cancer cells are injected in a tail vein assay, knock down of CD49f resulted in decreased metastasis to the lung [237]. These combined data suggest an important role for CD49f not only in promoting stem-like activity in vitro, primary tumor growth and TIC potential in vivo, but also in promoting lung metastases in multiple models of breast cancer.

Integrins such as ITGA6/CD49f are essential for invasion and migration of tumor cells during metastasis. In order for cancer cells to break through a target tissue's basal membrane, they must attach to the tissue's cell surface receptors, most of which are integrins. Integrins are crucial, not only for physically tethering cells to the matrix, but also for sending and receiving molecular signals that regulate these processes during tumorigenesis. In breast cancer, specifically, an increase in ITGA6 corresponds to an increase in intermediate filaments keratin and vimentin to increase tumor cell migratory potential [238]. Additionally, in advanced breast carcinomas ITGA6, in combination with integrin beta 4 (ITGB4), has been found at the leading edge of invasive cells, where it cooperates with ErbB2 and ErbB3 to promote aberrant cell signaling [239]. Moreover, 
ITGA6/ITGB4 regulates expression of SPARC (secreted protein acidic and rich in cysteine), which is involved with matrix remodeling and enhanced SPARC expression promotes invasion [240].

To determine if our experimental results have any direct relevance to human disease, we performed bioinformatic analyses of publically available data sets in regard to ITGA6 mRNA expression among human breast cancer subtypes. The basal-like subtype of breast cancer, including TNBCs, have the highest levels of ITGA6 mRNA as compared to all other subtypes. Additionally, high ITGA6 expression positively correlated with high $H I F 1 A$ expression across all subtypes of breast cancer. Whole genome expression profiling of the breast cancer subtypes has revealed that TNBCs, and to a lesser extent, HER2-enriched tumors, have an enriched hypoxic gene signature as compared to luminal breast cancers [92]. The HIF pathway has also been identified as one of three key pathways enriched in basal versus luminal cancers in The Cancer Genome Atlas data set [93]. In addition, in a variety of solid tumors, HIF-1 $\alpha$ or HIF-2 $\alpha$ expression has been linked to increased activity of TICs [156]. As HIF protein expression is enriched in TNBCs, and HIFs regulate breast TICs, the hypoxic response influence may directly influence the increased aggressive nature and chemotherapy resistance phenotypes observed in TNBCs. Finally, elevated expression of ITGA6 is prognostic, predicting a decrease in overall and recurrence free survival patients with breast cancer, indicating its potential usefulness as a biomarker to predict metastasis. 


\section{CHAPTER 5. IDENTIFYING NOVEL OR UNDERSTUDIED HIF- REGULATED GENES}

\section{Rationale}

As discussed in Chapter 3 and in [127], we have extensively characterized HIF-1 $\alpha$ wild type (WT) and knockout (KO) mammary tumor epithelial cells (MTECs) derived from late stage, ER-/PR-/Neu+ carcinomas of MMTV-PyMT transgenic mice also harboring Hifla floxed alleles. Two microarray screens were performed to identify HIF$1 \alpha$-regulated candidate genes that may promote tumor growth and/or lung metastasis. Differential gene expression profiles were compared between WT and KO cells cultured at normoxia $\left(21 \% \mathrm{O}_{2}\right)$ and hypoxia $\left(0.5 \% \mathrm{O}_{2}\right)$ and between end-stage WT and $\mathrm{KO}$ mammary tumors derived from transplantation of the parental cell lines into the mammary fat pad. Several genes were down-regulated in response to deletion of Hifla that are predicted to regulate primary tumor growth and/or the metastatic switch. HIF-1 $\alpha$ is known to promote multiple steps in metastasis and we found HIF-1 regulates expression of many uncharacterized hypoxia-inducible genes in PyMT mammary tumor cells. Characterization of the roles that genes downstream of HIF-1 play in driving metastatic potential may identify additional pathways that are amenable to therapeutic intervention and that may be more specific to the metastatic program than globally repressing HIF-1 activity using HIF inhibitors.

\section{Methods}

\section{Gene Expression Microarray Analysis}

PyMT HIF-1 $\alpha$ WT and KO cells were plated in triplicate in standard growth media (Chapter 3). As we found that addition of EGF to the culture media super induces HIF-1 $\alpha$ expression, even at normoxia (Figure 3-2, [127]), cells were cultured either in the presence of absence of $10 \mathrm{ng} / \mathrm{mL}$ mouse recombinant EGF (Invitrogen). Each condition (WT $\pm \mathrm{EGF}, \mathrm{KO} \pm \mathrm{EGF}$ ) was plated in triplicate (technical replicates) for both normoxic and hypoxic exposure (summarized in Table 5-1). At approximately $90 \%$ confluence, cells were placed at either normoxia $\left(21 \% \mathrm{O}_{2}\right)$ or hypoxia $\left(0.5 \% \mathrm{O}_{2}\right)$ for 6 hours. This time point was chosen as HIF-1 $\alpha$ protein expression begins to peak at 3 hours of hypoxic exposure (Figure 3-3) and mRNA levels of classic hypoxia-inducible genes, such as Vegf and Glutl are highest at 6 hours. Additionally, three HIF-1 $\alpha$ WT and three HIF- $1 \alpha \mathrm{KO}$ end-stage tumors were harvested and size matched based on volume and weight. Total RNA was harvested from flash frozen cells and from end-stage tumors as described in Chapter 3. All samples were analyzed for RNA integrity (RIN) by an Agilent Bioanalyzer and only samples with a RIN greater than 9 were hybridized on a MouseRef-8 v2.0 Expression BeadChip Kit (Illumina) at the UTHSC Molecular Resource Center. This BeadChip contains approximately 25,600 well-annotated RefSeq transcripts for $\sim 19,100$ unique genes in the mouse genome. Raw data was transformed to 
Table 5-1. Summary of culture conditions used for cells harvested for microarray profiling.

\begin{tabular}{lcc}
\hline \multicolumn{1}{c}{ Genotype } & O Exposure & EGF Addition \\
\hline Hifla WT & Normoxia, $6 \mathrm{hrs}$ & None \\
Hifla WT & Hypoxia, $6 \mathrm{hrs}$ & None \\
Hifla WT & Normoxia, $6 \mathrm{hrs}$ & $10 \mathrm{ng} / \mathrm{mL}$ \\
Hifla WT & Hypoxia, $6 \mathrm{hrs}$ & $10 \mathrm{ng} / \mathrm{mL}$ \\
Hifla KO & Normoxia, $6 \mathrm{hrs}$ & None \\
Hifla KO & Hypoxia, $6 \mathrm{hrs}$ & None \\
Hifla KO & Normoxia, $6 \mathrm{hrs}$ & $10 \mathrm{ng} / \mathrm{mL}$ \\
Hifla KO & Hypoxia, $6 \mathrm{hrs}$ & $10 \mathrm{ng} / \mathrm{mL}$ \\
\hline
\end{tabular}

the $\log _{2}$-scale, and intensity values were quantile normalized using Expander [free download, Tel-Aviv University, Israel (2003)]. Normalized log-scale intensity values were analyzed for differential expression using the Expander software. Seven pairwise comparisons were made from the cell data (Table 5-2) in order to obtain a complete image of gene changes either in the presence or absence of EGF and in response to exposure to hypoxia. All genes with a mean fold differential $\geq 2$, and with a $p$-value less than 0.05 among technical replicates were included in gene lists. However, data obtained from the three tumors/genotype revealed that one WT and one KO tumor were distinct outliers compared to the other tumors for nearly all of the genes. These tumors were removed from the analysis and only two tumors/genotype were compared, therefore, mean fold changes did not have $p$-values associated with them. Additionally, since gene expression changes in vivo were not as robust as in vitro, a fold change cut off of 1.5 was used to assess gene expression changes in the tumors.

\section{Functional Analysis}

In order to obtain a comprehensive set of functional annotations from the list of genes we identified as being differentially regulated, we used DAVID (the Database for Annotation, Visualization and Integrated Discovery, http://david.abcc.ncifcrf.gov). From this analysis we were able to identify functionally related gene groups. Additionally, the KEGG database (Kyoto Encyclopedia of Genes and Genomes, http://www.genome.jp/kegg/) was used to place predicted HIF-1 $\alpha$-target genes into molecular pathways. Gene Set Enrichment Analysis (GSEA, http://www.broadinstitute.org/gsea/index.jsp) was used to determine if the gene sets identified with our arrays significantly correlated with other published pathway and 
Table 5-2. All pairwise comparisons from datasets from cells.

\begin{tabular}{lcc}
\hline \multicolumn{1}{c}{ Comparison } & Data Set 1 & Data Set 2 \\
\hline 1 & WT Normoxia plus EGF & WT Normoxia without EGF \\
2 & WT Normoxia without EGF & WT Hypoxia without EGF \\
3 & WT Hypoxia plus EGF & WT Hypoxia without EGF \\
4 & WT Normoxia plus EGF & KO Normoxia plus EGF \\
5 & WT Normoxia without EGF & KO Normoxia without EGF \\
6 & WT Hypoxia plus EGF & KO Hypoxia plus EGF \\
7 & WT Hypoxia without EGF & KO Hypoxia without EGF \\
\hline
\end{tabular}

functional gene sets. Dr. Fan was instrumental in advising our lab in how to access and utilize these tools.

\section{Results}

\section{Effects of EGF on HIF-1 $\alpha$-Dependent Gene Expression}

As discussed in Chapter 3 (Figure 3-2), exposing PyMT MTECs to EGF increases HIF-1 $\alpha$ protein levels at normoxia, and further increases HIF- $1 \alpha$ levels at hypoxia as compared to cells grown in $2 \%$ FBS alone. When WT normoxia plus EGF samples were compared to WT normoxia without EGF, 99 genes were found to be up regulated in cells treated with EGF. When exposed to 6 hours of hypoxia this number decreased to only 83 genes that were-up regulated in the presence of EGF. This result is interesting as it was expected the combination of hypoxic exposure and the presence of EGF would increase the number of up-regulated genes versus EGF alone. Furthermore, comparison of the genes in each of these groups revealed only 6 genes that overlapped (Figure 5-1A), further suggesting that EGF treatment primarily regulates genes in a HIF$\alpha /$ hypoxia-independent manner. In the absence of EGF, hypoxic exposure up-regulated 141 genes and only a few of these genes (Figure 5-1B) overlapped with genes in either of the previous two comparisons, resulting in 128 genes that were hypoxia-inducible only, and 90 that were EGF-inducible only.

At normoxia, 95 genes were found to be up regulated in HIF-1 $\alpha$ WT versus HIF$1 \alpha \mathrm{KO}$ cells in the presence of EGF (Figure 5-2A). However, in the absence of EGF 308 genes were found to be up-regulated in WT versus KO cells. Comparing the two groups there were 61 genes in common, suggesting that most genes are HIF-1 $\alpha$, but not EGFdependent. As expected, upon hypoxic exposure the number of genes up-regulated in WT 

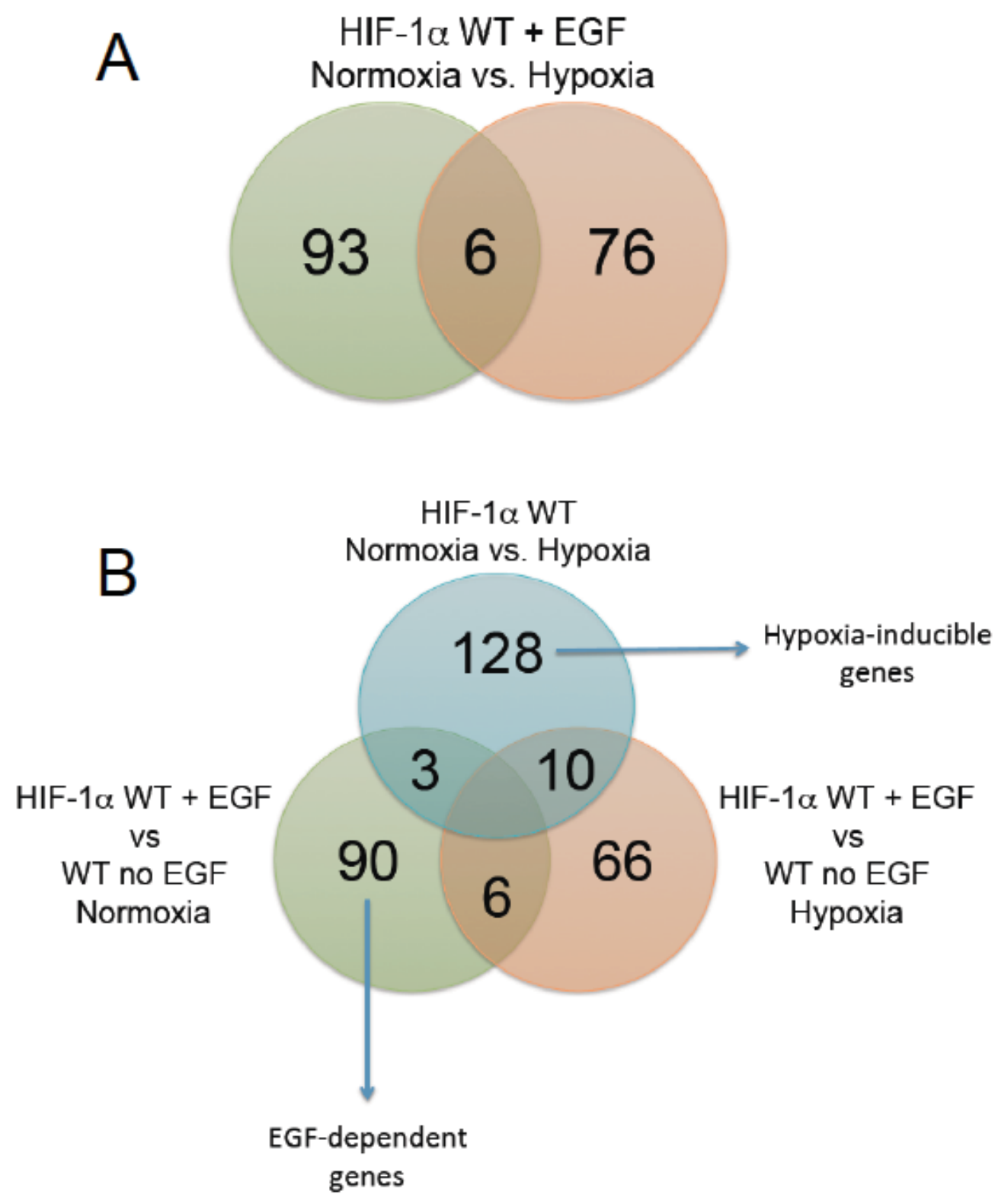

Figure 5-1. Comparison of up-regulated genes in HIF-1 $\alpha$ WT cells in the presence of EGF \pm hypoxia. 


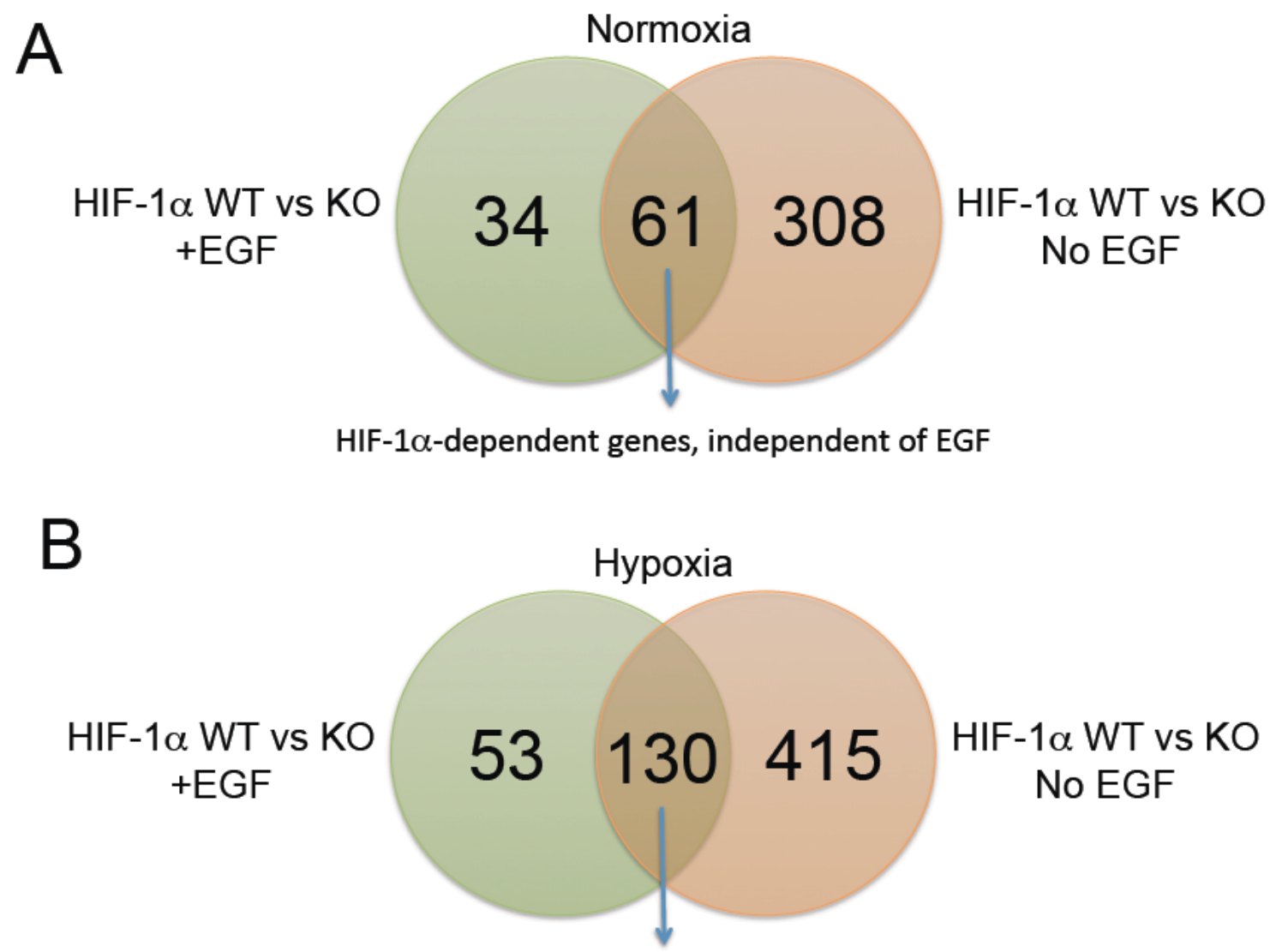

HIF-1 $\alpha$-dependent, hypoxia-inducible genes, independent of EGF

Figure 5-2. HIF-1 $\alpha$-dependent genes in the presence and absence of EGF and hypoxia. 
versus KO cells increases from 95 to 183 in the presence of EGF and from 308 to 545 in the absence of EGF, with 130 overlapping genes (Figure 5-2B). We have shown that not only does EGF treatment induce HIF-1 $\alpha$ protein stabilization, but that it also induces gene expression independent of HIF- $1 \alpha$. Therefore, the observation that more genes are differentially regulated in the absence of EGF could be a result of the presence of EGF masking the full effects of HIF-1 $\alpha$-dependent gene expression. Therefore, we chose to focus on the data generated from cells cultured without EGF for further comparisons. Since the cells are cultured in low serum without additional growth factors, we felt that the comparison 7 data set would reveal genes most dependent upon HIF-1 in the hypoxic environment.

\section{HIF-1 $\alpha$-Dependent Gene Expression in PyMT MTECs and Tumors}

Comparing HIF-1 $\alpha$ WT to HIF-1 $\alpha$ KO cells with no EGF, we found 512 differentially regulated genes at normoxia and 820 differentially regulated genes at hypoxia (Figure 5-3). At normoxia, there were 369 genes that were up-regulated in WT cells and 143 genes that were down-regulated. At hypoxia, there were 545 genes up regulated in WT cells and 275 genes that were down regulated.

Analysis of the data obtained from tumors revealed that 696 genes are downregulated in WT tumors and 782 genes up regulated in WT tumors. Comparing up regulated genes in cells and tumors, 68 genes were found to be in common. For downregulated genes, 26 genes were in common (Figure 5-4). These numbers suggest that the in vivo tumor microenvironment plays a large role in regulation of HIF-1-dependent gene expression. Table 5-3 lists the top 10 up and down regulated genes that are in common between cells and tumors. Some of the genes identified as up-regulated are already known to be HIF target genes, such as pyruvate dehydrogenase kinase 1 (Pdkl) [241]. Others, such as Creatine Kinase brain isoform $(C k b)$, Vitamin D receptor $(V d r)$, and Embigin $(E m b)$, do not have a clear published role as to their relationship with HIF or the hypoxic response. Differential expression of $V d r$ was not validated at the protein level (data not shown). We decided to focus on $C k b$ due to dearth of publications on $E m b$, and a lack of robustly tested commercially available reagents (such as antibodies to Emb).

DAVID functional annotation clustering analysis revealed the 7 top functional pathways dependent upon HIF-1 (Table 5-4). Specifically, cytokine and growth factor signaling pathways are strongly affected; both are important pathways not only in cell growth and survival, but also in tumor growth and progression. Not surprisingly, angiogenesis is also enriched as the HIFs regulate a main angiogenesis gene, VEGF [175, 242]. Additionally, extracellular matrix, cell-cell adhesion and cell migration and motility are also enriched pathways, and each is important for tumor growth, progression and metastasis [243] [101, 244]. KEGG pathway analysis revealed similar pathways to DAVID (Table 5-5) and also implicated additional cancer pathways that were enriched in our HIF-1 $\alpha$ differentially regulated gene set. Gene set enrichment analysis (GSEA) correlated with DAVID and KEGG and showed that both cell cycle and EMT pathways are enriched (Table 5-6). In addition, GSEA suggested that breast cancer pathways and 


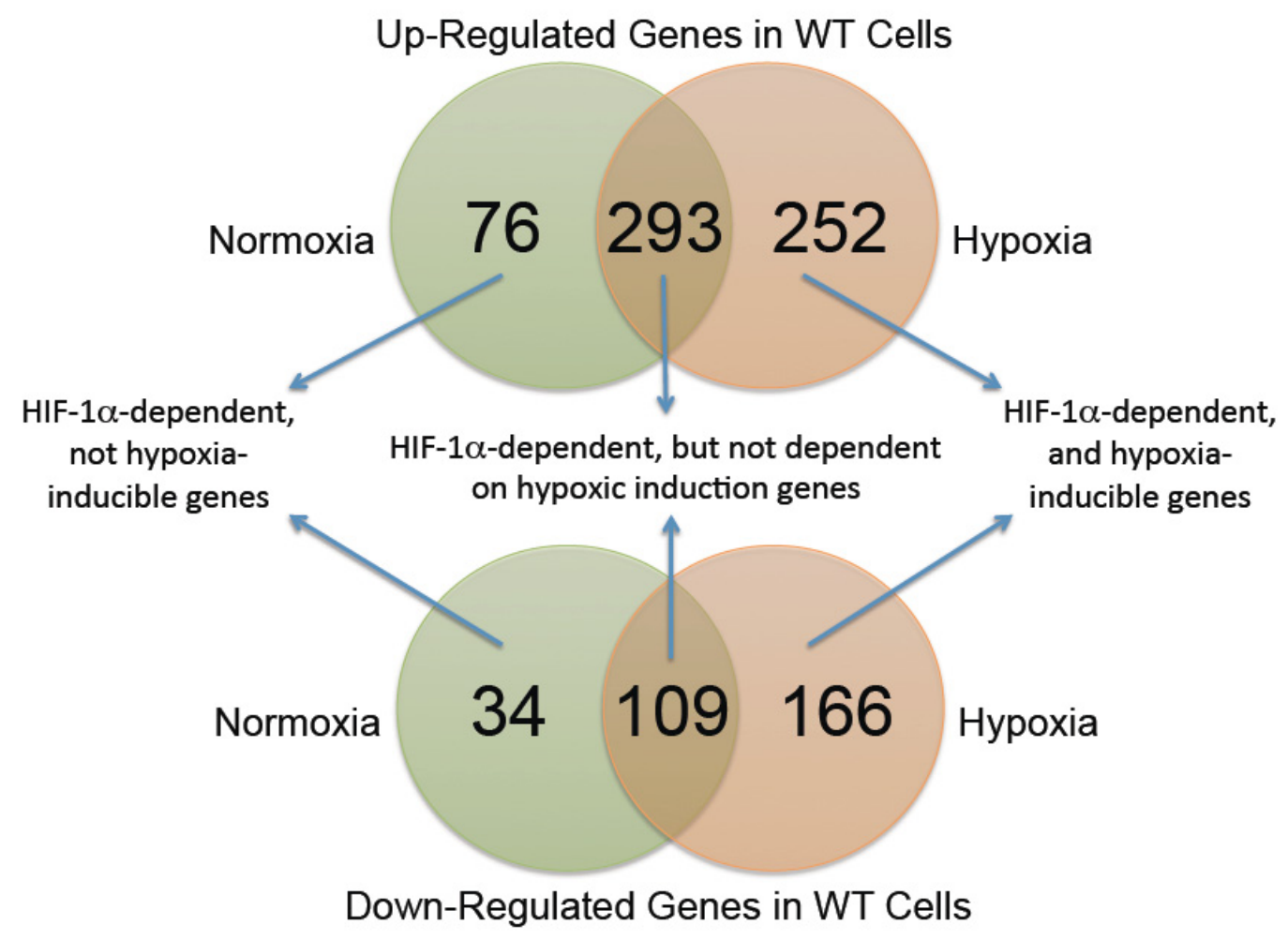

Figure 5-3. Differentially regulated genes between HIF-1 $\alpha$ WT and KO cells at normoxia and hypoxia. 


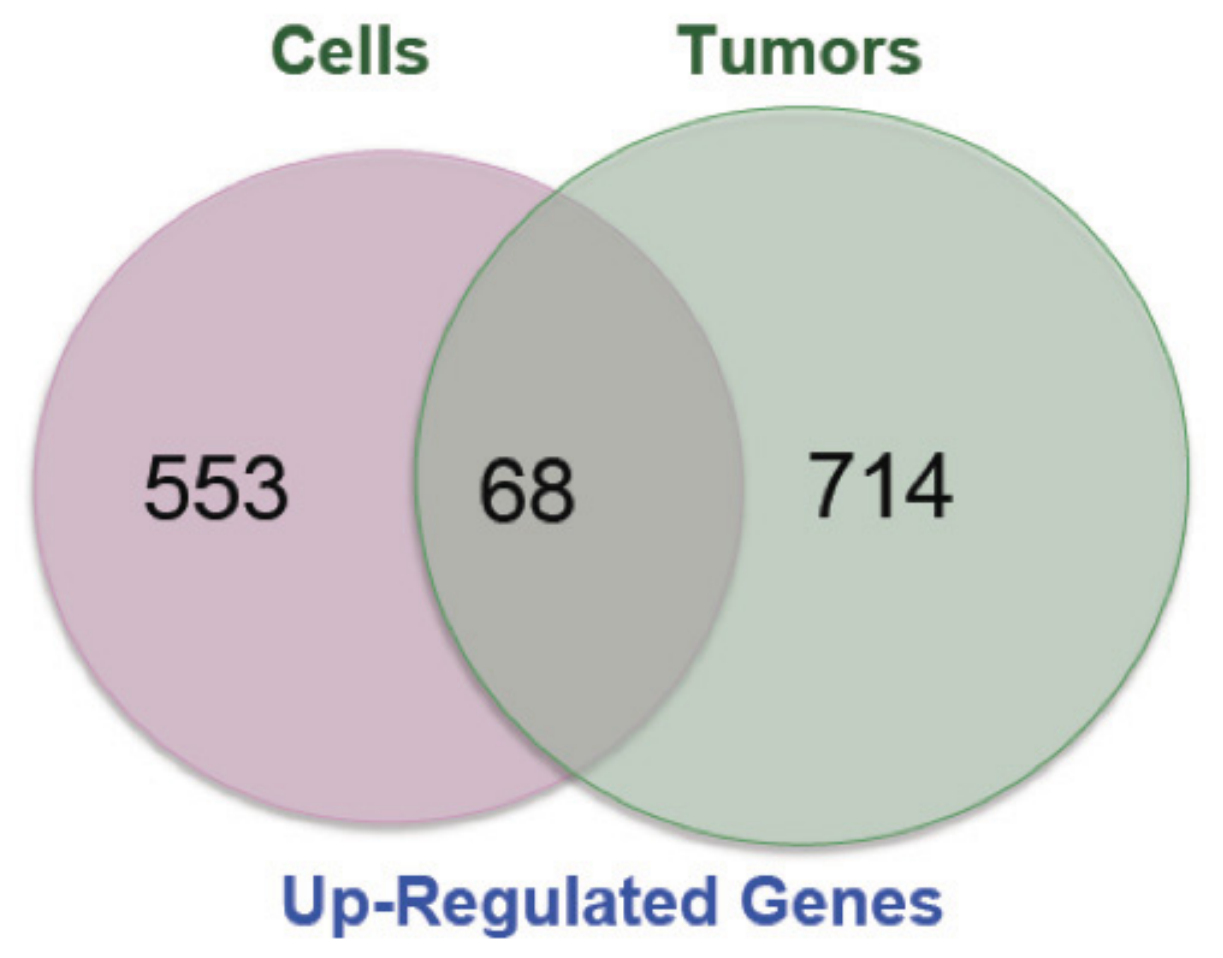

\section{Cells Tumors}

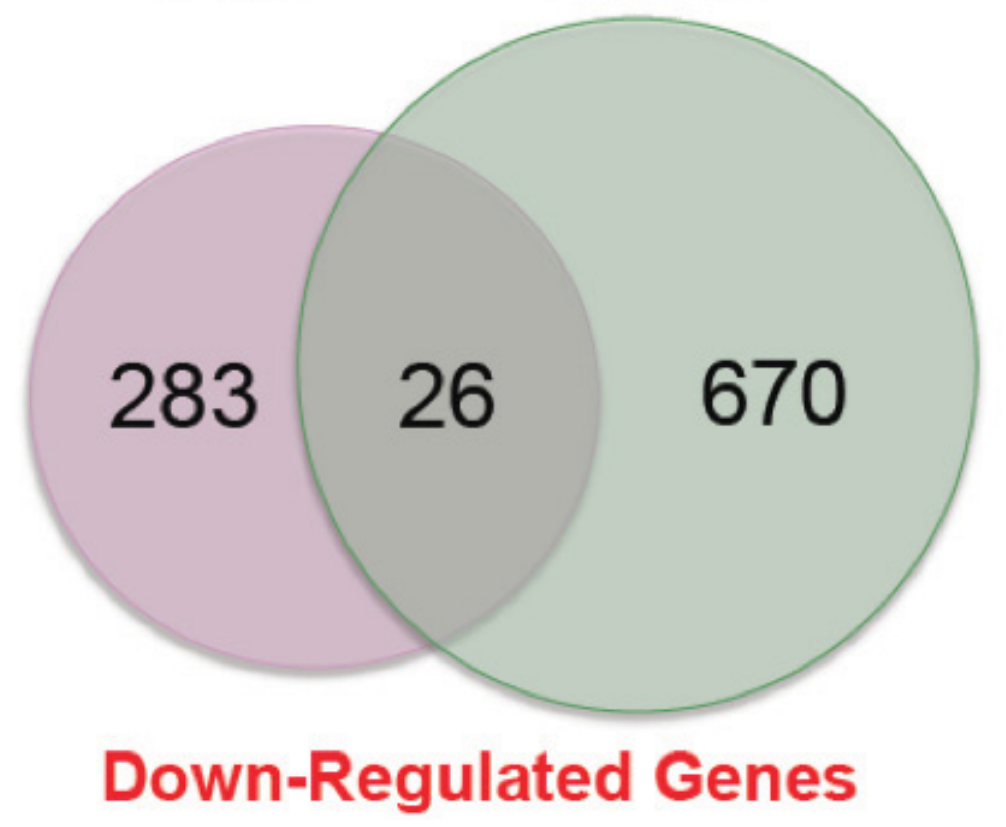

Figure 5-4. Differentially regulated genes in common between PyMT MTECs and end stage tumors. 
Table 5-3. Top 10 differentially regulated genes between cells and tumors.

\begin{tabular}{|c|c|c|c|c|c|}
\hline \multirow[b]{2}{*}{$\begin{array}{c}\text { Genes Up- } \\
\text { Regulated in WT }\end{array}$} & \multicolumn{2}{|c|}{ Fold Change } & \multirow[b]{2}{*}{$\begin{array}{c}\text { Genes Down-Regulated } \\
\text { in WT }\end{array}$} & \multicolumn{2}{|c|}{ Fold Change } \\
\hline & $\begin{array}{l}\text { Cell } \\
\text { FC }\end{array}$ & $\begin{array}{l}\text { Tumor } \\
\text { FC }\end{array}$ & & $\begin{array}{l}\text { Cell } \\
\text { FC }\end{array}$ & $\begin{array}{l}\text { Tumor } \\
\text { FC }\end{array}$ \\
\hline $\begin{array}{l}\text { Creatine kinase } \\
\text { brain isoform }(C k b)\end{array}$ & 23.64 & 1.50 & $\begin{array}{l}\text { Cation transport regulator } \\
\text { homolog } 1 \text { (Chacl) }\end{array}$ & 10.32 & 1.76 \\
\hline $\begin{array}{l}\text { Vitamin D receptor } \\
(V d r)\end{array}$ & 16.76 & 2.35 & $\begin{array}{l}\text { Caspase recruitment } \\
\text { domain-containing } \\
\text { protein } 14(\text { Card14) }\end{array}$ & 6.12 & 1.69 \\
\hline Embigin $(E m b)$ & 11.71 & 2.40 & B-cell linker $(B \ln k)$ & 5.82 & 1.23 \\
\hline $\begin{array}{l}\text { Pyruvate } \\
\text { dehydrogenase } \\
\text { kinase, isozyme } 1 \\
(P d k l)\end{array}$ & 8.27 & 1.60 & $\begin{array}{l}\text { DTW domain containing } \\
1(D t w d l)\end{array}$ & 5.26 & 1.60 \\
\hline $\begin{array}{l}\text { Nuclear prelamin A } \\
\text { recognition factor } \\
(\text { Narf) }\end{array}$ & 8.04 & 3.21 & $\begin{array}{l}\text { Bifunctional } \\
\text { methylenetetrahydrofolate } \\
\text { dehydrogenase/cyclo- } \\
\text { hydrolase, mitochondrial } \\
(M t h f d 2)\end{array}$ & 4.93 & 1.51 \\
\hline $\begin{array}{l}\text { Peptidyl-prolyl cis- } \\
\text { trans isomerase C } \\
(\text { Ppic })\end{array}$ & 7.23 & 2.58 & $\begin{array}{l}\text { Insulin-like growth factor } \\
\qquad 2(\operatorname{Igf} 2)\end{array}$ & 3.97 & 1.53 \\
\hline $\begin{array}{l}\text { Insulin-like growth } \\
\text { factor-binding } \\
\text { protein } 4(\operatorname{Ig} f b p 4)\end{array}$ & 6.57 & 3.30 & Glypican (Gpcl) & 3.58 & 1.54 \\
\hline $\begin{array}{l}\text { A disintegrin and } \\
\text { metalloproteinase } \\
\text { with } \\
\text { thrombospondin } \\
\text { motifs } 4 \text { (Adamts } 4 \text { ) }\end{array}$ & 5.74 & 2.03 & $\begin{array}{l}\text { DNA damage-inducible } \\
\text { transcript } 3 \text { (Ddit } 3 \text { ) }\end{array}$ & 3.20 & 3.07 \\
\hline $\begin{array}{l}\text { Milk fat globule- } \\
\text { EGF factor } 8 \text { protein } \\
(M f g e 8)\end{array}$ & 5.39 & 2.44 & $\begin{array}{l}\text { Coiled-coil-helix-coiled- } \\
\text { coil-helix domain } \\
\text { containing } 10 \\
(C h c h d 10 / N d g 2)\end{array}$ & 3.07 & 1.56 \\
\hline $\begin{array}{l}\text { Surfactant, } \\
\text { pulmonary- } \\
\text { associated protein D } \\
(\text { Sftpd })\end{array}$ & 5.30 & 2.84 & $\begin{array}{l}\text { Nuclear pore } \\
\text { glycoprotein-210 } \\
(\text { Nup } 210)\end{array}$ & 2.83 & 1.58 \\
\hline
\end{tabular}

$\mathrm{FC}=$ Fold Change 
Table 5-4. DAVID functional analysis of differentially regulated HIF-1 $\alpha$ genes.

\begin{tabular}{lc}
\hline \multicolumn{1}{c}{ DAVID Annotation Cluster } & Enrichment Score \\
\hline Cytokine and Growth Factor Signaling & 14.32 \\
Extracellular Matrix & 8.45 \\
Blood Vessel Development/Angiogenesis & 3.2 \\
Cell-Cell Adhesion & 2.9 \\
Growth Factor Binding & 2.51 \\
Glucose Metabolism & 2.45 \\
Cell Migration and Motility & 2.11 \\
\hline
\end{tabular}

Table 5-5. Top 10 KEGG Pathway Analysis.

\begin{tabular}{ll}
\hline \multicolumn{1}{c}{ KEGG Pathway } & $\boldsymbol{p}$-Value \\
\hline ECM-receptor interaction & $2.50 \mathrm{E}-04$ \\
Cytokine-cytokine receptor interaction & $4.20 \mathrm{E}-04$ \\
Focal adhesion & $2.60 \mathrm{E}-03$ \\
Pancreatic cancer & $4.10 \mathrm{E}-03$ \\
Dilated cardiomyopathy & $6.90 \mathrm{E}-03$ \\
Alanine, aspartate and glutamate metabolism & $9.20 \mathrm{E}-03$ \\
Melanogenesis & $1.20 \mathrm{E}-02$ \\
Pathways in cancer & $1.50 \mathrm{E}-02$ \\
Metabolism of xenobiotics by cytochrome P450 & $2.50 \mathrm{E}-02$ \\
Galactose metabolism & $2.90 \mathrm{E}-02$ \\
\hline
\end{tabular}


Table 5-6. GSEA Analysis of HIF-1 $\alpha$ regulated genes enriched in WT cells and tumors.

\begin{tabular}{|c|c|c|}
\hline Gene Set Name (MsigDB) & Description & $p$-Value \\
\hline RAS_ONCOGENIC_SIGNATURE & $\begin{array}{l}\text { Genes selected in supervised } \\
\text { analyses to discriminate cells } \\
\text { expressing activated H-Ras } \\
\text { oncogene from control cells } \\
\text { expressing GFP. }\end{array}$ & $8.60 \mathrm{E}-11$ \\
\hline BRCA_ER_NEG & $\begin{array}{l}\text { Genes whose expression is } \\
\text { consistently negatively correlated } \\
\text { with estrogen receptor status in } \\
\text { breast cancer - higher expression } \\
\text { is associated with ER-negative } \\
\text { tumors }\end{array}$ & 7.40E-09 \\
\hline EMT_UP & $\begin{array}{l}\text { Up-regulated during the } \\
\text { TGFbeta-induced epithelial-to- } \\
\text { mesenchymal transition (EMT) } \\
\text { of Ras-transformed mouse } \\
\text { mammary epithelial (EpH4) cells } \\
\text { (EMT is representative of late- } \\
\text { stage tumor progression and } \\
\text { metastasis) }\end{array}$ & $2.11 \mathrm{E}-08$ \\
\hline ABBUD_LIF_UP & $\begin{array}{l}\text { Genes that increased after LIF } \\
\text { treatment }(10 \mathrm{ng} / \mathrm{ml} \text {, overnight }) \\
\text { in AtT } 20 \text { cells }\end{array}$ & $9.04 \mathrm{E}-06$ \\
\hline MAPK_SIGNALING_PATHWAY & $\begin{array}{l}\text { Genes involved in MAPK } \\
\text { signaling pathway }\end{array}$ & $1.09 \mathrm{E}-05$ \\
\hline HSC and PROGENITORS_SHARED & $\begin{array}{l}\text { Up-regulated in mouse } \\
\text { hematopoietic stem cells and } \\
\text { progenitors from both adult bone } \\
\text { marrow and fetal liver (Cluster } \\
\text { iii, HSC and Progenitors Shared) }\end{array}$ & $1.15 \mathrm{E}-05$ \\
\hline
\end{tabular}


Table 5-6. (Continued).

\begin{tabular}{|c|c|c|}
\hline Gene Set Name (MsigDB) & Description & $p$-Value \\
\hline EMT_DN & $\begin{array}{l}\text { Down-regulated during the } \\
\text { TGFbeta-induced epithelial-to- } \\
\text { mesenchymal transition (EMT) } \\
\text { of Ras-transformed mouse } \\
\text { mammary epithelial (EpH4) cells } \\
\text { (EMT is representative of late- } \\
\text { stage tumor progression and } \\
\text { metastasis) }\end{array}$ & $3.53 \mathrm{E}-05$ \\
\hline BRCA_ER_POS & $\begin{array}{l}\text { Genes whose expression is } \\
\text { consistently positively correlated } \\
\text { with estrogen receptor status in } \\
\text { breast cancer - higher expression } \\
\text { is associated with ER-positive } \\
\text { tumors }\end{array}$ & $6.93 \mathrm{E}-05$ \\
\hline HYPOXIA_REVIEW & $\begin{array}{c}\text { Genes known to be induced by } \\
\text { hypoxia }\end{array}$ & $9.28 \mathrm{E}-05$ \\
\hline WNT_SIGNALING_PATHWAY & $\begin{array}{c}\text { Genes involved in Wnt signaling } \\
\text { pathway }\end{array}$ & $5.96 \mathrm{E}-03$ \\
\hline HSA04110_CELL_CYCLE & Genes involved in cell cycle & $1.15 \mathrm{E}-02$ \\
\hline
\end{tabular}


hypoxia pathways are enriched in WT cells and tumors.

\section{Discussion}

Microarray analysis of HIF- $1 \alpha$ WT and KO cells and tumors produced data implicating hundreds of genes as being differentially regulated by HIF-1 $\alpha$ expression in PyMT late stage carcinomas and cell lines. Some of the genes identified have long been known to be HIF-1 $\alpha$ target genes, such as Vegf, Glut1, Phd3/Egln3, Aldoc, Ldha, Gapdh and many more [24]. Furthermore, genes were identified that have recently been shown to be direct HIF-1 $\alpha$-target genes, including Axl [245], Angptl4 [72], and multiple jumonji-domain histone demethylases [246]. Additionally, analysis of the gene lists revealed enrichment of several genes in WT cells that are either involved in stem cell maintenance, or are commonly used stem cell surface markers including CD133/Prom 1 [117], which has been shown to a HIF-1 $\alpha$ target gene, Gata3 [247], Wnt signaling pathway genes such as Wnt10a, Wnt5b, Lrp5, and Fzd7 [248], Notch pathway genes such as Dlll and Heyl [164], and Itga6/CD49f. However, there are still many genes on these lists that have yet to be identified as direct HIF-1 $\alpha$ target genes, or to have a direct implication in HIF-1 $\alpha$-dependent tumor growth and progression. Investigating the role of one of these genes, $C k b$, in breast tumor growth and development is the focus of the next chapter. 


\section{CHAPTER 6. CREATINE KINASE B (CKB) ACTIVITY IS HIF-1- DEPENDENT AND IS ESSENTIAL FOR PRIMARY TUMOR GROWTH, CELL INVASION AND METASTASIS TO THE LUNG}

\section{Introduction}

As we have previously shown, HIF-1 $\alpha$ expression is required for mammary tumor growth and metastasis in the MMTV-PyMT mouse model of breast cancer [127]. In order to identify genes that are required for this phenotype that act downstream of HIF-1 $\alpha$, we performed microarray profiling on both Hifla WT and KO cells as well as late stage mammary carcinomas derived from these cells. We found 94 genes differentially regulated by HIF-1 $\alpha$ in both settings (Chapter 5). Many of these genes, including Vegf, Egln3 (PHD3), Aldoc and Pdk1 were known HIF-1 $\alpha$ target genes and are involved in cancer cell migration and invasion, tumor initiation and growth, and metastasis $[61,154]$ $[72,116]$. One of the genes we found to be the most differentially up-regulated in WT cells and tumors is the enzyme creatine kinase brain isoform $(C k b)$.

\section{The Creatine Kinase System}

$\mathrm{CKB}$ is a member of a family of creatine kinases (CKs) that reversibly transfer a high-energy phosphate group between ATP and creatine to generate phosphocreatine (PCr) and ADP (Figure 6-1). There are two cytoplasmic CK isoforms, CKB and CKM (muscle isoform), which dimerize to form CKB-B or CKM-M homodimers or a CKB-M heterodimer, and two mitochondrial isoforms, CKMT1 and CKMT2 [249]. CKs are expressed at high levels in tissues with high and rapidly changing energy demand, such as brain and muscle [250]. Ischemia and hypoxia are two conditions in which ATP production via oxidative phosphorylation is inhibited due to the lack of oxygen; therefore, $\mathrm{CK}$ metabolism supplements energy produced by oxidative phosphorylation and glycolysis, i.e., PCr serves an energy reservoir for CKs to re-generate ATP from ADP in highly metabolic tissues [251].

\section{CKB and Cancer}

Although named "brain" isoform, CKB is expressed in virtually all cell types, and $\mathrm{CKB}$ over-expression has been documented in a variety of solid tumors as compared to normal cells [251]. Particularly, CKB has been found to be overexpressed in several types of cancers including breast cancer, colorectal cancer, colon cancer, and ovarian cancer [252-256]. Although not much is known about the direct function of CKB in cancer progression and metastasis, one study found that $C K B$ knockdown inhibited cell proliferation of the ovarian cancer cell line Skov3 and induced apoptosis under hypoxia or hypoglycemic conditions [257]. These data suggest that CKB is linked to oxygen consumption and glycolysis. The authors also found that CKB depletion sensitized Skov3 cells to chemotherapeutic agents. Another study suggested that the role CKB plays in 


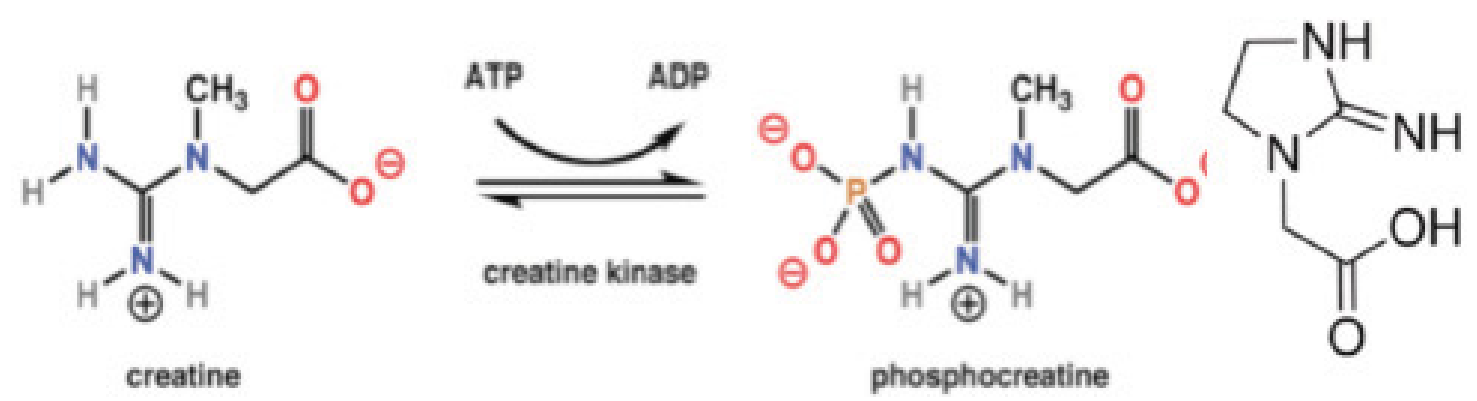

Figure 6-1. Overview of creatine kinase enzymatic activity.

Creatine kinase catalyzes the reversible reaction to generate phosphocreatine (right), or to generate creatine (left) from phosphocreatine to produce ATP.

Image reproduced from Sigma-Aldrich, Cyclocreatine 377627_technical datasheet.

cancer progression could be its localization along actin filaments within the cell, facilitating cell spreading and migration [258], though this hypothesis was not tested in vivo.

Inhibition of CKB by the phosphocreatine analog cyclocreatine in preclinical models. In 1994, a variety of creatine analogs were synthesized and analyzed for their ability to block ATP production through the CK system [259]. Several analogs were found to be active, however, cyclocreatine $(\mathrm{cCr})$ was the most active, resulting in 160fold less efficient ATP production through CK [260]. In initial studies of the effect of cCr as an anti-cancer agent, in 1993, Lillie et al found that not only did cCr inhibit tumor cell growth in vitro in a variety of cancer cell lines, but it also decreased the rate of tumor growth in animals transplanted with cervical cancer line ME- 180 that were fed $1 \% \mathrm{cCr}$ daily [261]. In 1995, in a rat mammary adenocarcinoma model, cCr given by daily IP injections starting at various intervals post-transplant resulted in a significant tumor growth delay in [262]. Furthermore, this delay was extended when $\mathrm{cCr}$ was given in combination with conventional cancer therapies such as Adriamycin and cyclophosphamide [262]. In addition, in 1998, an in vitro study of the prostate cancer cell line, DU-145, which expresses high endogenous levels of CKB, showed that treatment with cCr markedly inhibited motility of the cells to various chemoattractants, as well as decreased invasion through a Matrigel matrix [263].

Cyclocreatine (cCr) in phase I studies. When administered in a phase I/II dose escalation study to terminal cancer patients by i.v. infusion, the primary adverse side effects of $\mathrm{cCr}$ therapy were reversible hypoglycemia and fluid retention; the maximum tolerated dose (MTD) was estimated to be $80 \mathrm{mg} / \mathrm{kg}$ [251]. Despite an initial burst of reports touting $\mathrm{cCr}$ as an anticancer agent in the 1990s [259, 261-263], no current clinical trials are testing $\mathrm{cCr}$ (recent clinicaltrials.gov query). However, a recent high-profile report that $\mathrm{cCr}$ therapy ameliorated neurological symptoms and improved cognition in a mouse model of X-linked retardation may spark renewed interest in clinical use of GMPgrade $\mathrm{cCr}$ [264]. A web search has revealed that at least four companies are developing 
cCr, or cCr analogs, in their pipelines (Pantox Laboratories, Avicena, ALR Industries, and Lumos Pharma). Of these, Lumos Pharma has FDA approval to test cCr (Lum-001) in clinical trails as an orphan drug to test whether Lum-001 can ameliorate mental retardation in patients with a creatine transport deficiency. In addition, Lumos has an inactive investigational new drug application with the FDA to use Lum-001 in solid tumors. We are in the process of contacting Lumos to establish a collaborative agreement to obtain Lum-001 for research purposes, and for possibly initiating new clinical trials for MBC patients who have failed conventional therapy.

Despite evidence that $\mathrm{cCr}$ reduces primary mammary tumor mass, the efficacy of $\mathrm{cCr}$ to block or to treat established metastatic breast cancer has not been systematically evaluated. Moreover, limited pre-clinical studies have evaluated $\mathrm{cCr}$ therapy using mouse models in which the tumors develop at the appropriate orthotopic site (i.e. mammary gland, rather than subcutaneous) and that will metastasize to distant organs from the breast in immunocompetent rodent models.

In this Chapter, I will show that HIF-1 $\alpha$ KO PyMT cells and KO tumors express significantly less CKB mRNA and protein than WT cells and tumors. In WT cells transduced with lentivirus expressing either of two independent $C k b$ shRNAs (Figure 6-2), there is a significant decrease in primary tumor growth, an increase in survival from lung metastases induced by primary tumor resection, as well as a decrease in the incidence of lung metastases induced by tail vein injection. In addition, the formation of lung metastases in the CKB WT cells introduced into the circulation by tail vein injections into FVB mice was blocked by daily treatment with $\mathrm{cCr}$ without any apparent gross or organ cytotoxicities. These data, along with the above previous clinical data, indicate that cyclocreatine may be not only a beneficial anti-cancer agent but also as a potent anti-metastatic agent in breast cancer.

\section{Methods}

\section{Cell Culture}

Hifla wild-type and knockout mammary tumor epithelial cells (MTECs) were established as previously described in Chapter 2 and in [127], and maintained in medium containing DMEM/F-12 (DMEM/F-12; Invitrogen, Carlsbad, CA, USA) with 2\% FBS. For subcultivation, cells were rinsed twice with Puck's A saline, then incubated for up to 60 minutes at $37^{\circ} \mathrm{C}$ in a $3: 1$ solution of dispase II $/ 0.25 \%$ trypsin reconstituted in Puck's A. All cells were grown either at normoxia in an air-jacketed $\mathrm{CO}_{2}$ incubator $\left(5 \% \mathrm{CO}_{2}\right.$; SANYO, Wood Dale, IL, USA) or at hypoxia $\left(0.5 \% \mathrm{O}_{2}, 5 \% \mathrm{CO}_{2}\right)$ in a multigas incubator (SANYO) in which $\mathrm{N}_{2}$ gas displaces $\mathrm{O}_{2}$. Cells were exposed acutely ( $\leq 6$ hours) or chronically ( $>6$ hours to several days) to hypoxia and were removed from chronic hypoxic exposure only for brief periods to change the media. Due to the sensitivity of the PyMT cells to immediate serum withdrawal, cells were slowly weaned to $0.5 \%$ FBScontaining medium by decreasing the percentage of FBS by $0.5 \%$ after every cell 


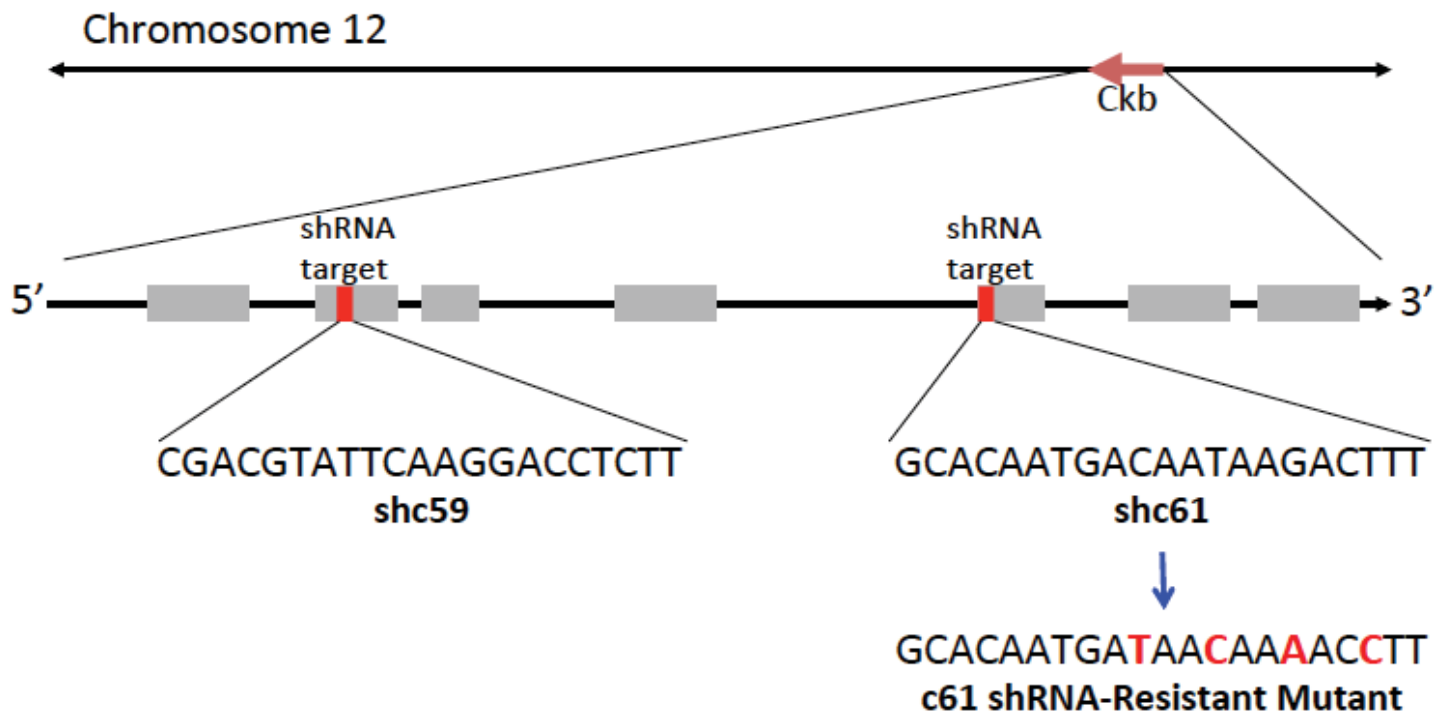

Figure 6-2. Structure of $\boldsymbol{C k b}$ and location of shRNA targeting sequences.

$C k b$ is located on Chromosome 12 of the mouse genome. The RNA transcript has 7 exons and the two shRNA targeting sequences we chose for knockdown are in different exons. shc59 is located in the middle of exon 2 and shc61 is located at the 5' end of exon 5. An shRNA resistant $C k b$ was recently generated by mutating 4 nucleic acids in the targeting sequence of shc61 (base pairs highlighted in red). These silent mutations do not change the protein sequence, or presumably the function of wild-type Ckb. 
subcultivation in order to perform invasion assays. Cells were then serum starved overnight for no longer than 16 hours prior to using in the invasion assay experiments.

\section{Generation of Ckb Cell Line Variants}

PyMT WT cells were used to create stable knockdown of $C k b$ via lentiviral transduction. A $C k b$ shRNA library containing 3 independent $C k b$ targeting sequences designed by The RNAi Consortium (TRC) and cloned into the pLKO.1 vector was purchased from Open Biosystems (RMM4534-EG12709, Figure 6-3A). Cells were transfected using a Nucleofector (Lonza, Basel Switzerland) with cell-specific kit $\mathrm{T}$ and program setting 24 to evaluate each construct's knockdown efficiency. Two of the three pLKO. 1 plasmid constructs produced $>75 \%$ knockdown (shc61 and shc59, Figure 6-2) by western blot and qRT-PCR. These two shRNA vectors were packaged into lentivirus particles by the UTHSC viral vector core for transduction of WT cells to generate stable $C k b$ knockdown (KD) cell lines following puromycin selection. WT cells were transduced with virus particles at an MOI of 50 with shc59, shc61 or empty vector (EV) and pools of positive clones (representing several hundred clones for each construct) were established and maintained in culture as described above with normal growth media plus $2 \mu \mathrm{g} / \mathrm{mL}$ puromycin.

To generate an shRNA-resistant $C k b$ expressing cell line, a full-length $C k b$ coding sequence was purchased from Open Biosystems (MMM1013-202770188, Accession: BC106109, Clone ID: 6395794) in the pCMV-Sport6 plasmid. Using the Not1 and Sal1 restriction enzyme site, $C k b$ was cloned into a modified entry vector, $\mathrm{pENTR} 4-\mathrm{FLAG}$ (Figure 6-3B) [265], purchased from Addgene (17423) to create an N-terminal FLAGtagged $C k b$. Using a site directed mutagenesis kit (QuikChange Lightning Multi SiteDirected Mutagenesis Kit, Agilent Technologies 210515), four silent mutations were introduced into the target sequence for shc61 (Figure 6-2) following the manufacturer's protocol. FLAG-tagged, WT Ckb containing mutant shc61 targeting sites was then shuttled into a modified destination lentiviral expression vector, pLenti- PGK-Neo (Figure 6-3C) [265], using LR recombinase technology (LR Clonase ${ }^{\circledR}$ II Plus, Invitrogen, 12538120). Vectors have been packaged into lentiviral particles by the UTHSC viral vector core, and experiments to transduce virus into PyMT KO cells as are in progress. Following transduction, pools will be selected using neomycin to select for transduced cells.

\section{Protein Extraction and Western Blotting}

Whole cell extracts (WCE) were prepared as described in chapter 4 and in [41]. WCE were resolved on $4 \%$ to $12 \%$ Tris-Base gels $(100 \mu \mathrm{g} / \mathrm{lane}$; Invitrogen) and transferred onto polyvinylidene fluoride (PVDF) or PVDF Fluorescent (PVDF-FL) membranes prior to blocking with $5 \%$ milk. Antibodies for CKB, $\beta$-tubulin, and SMA detection were purchased from Epitomics (2830), Abcam (6046) and Sigma-Aldrich (A2547), respectively. Primary antibodies were probed with secondary antibodies 

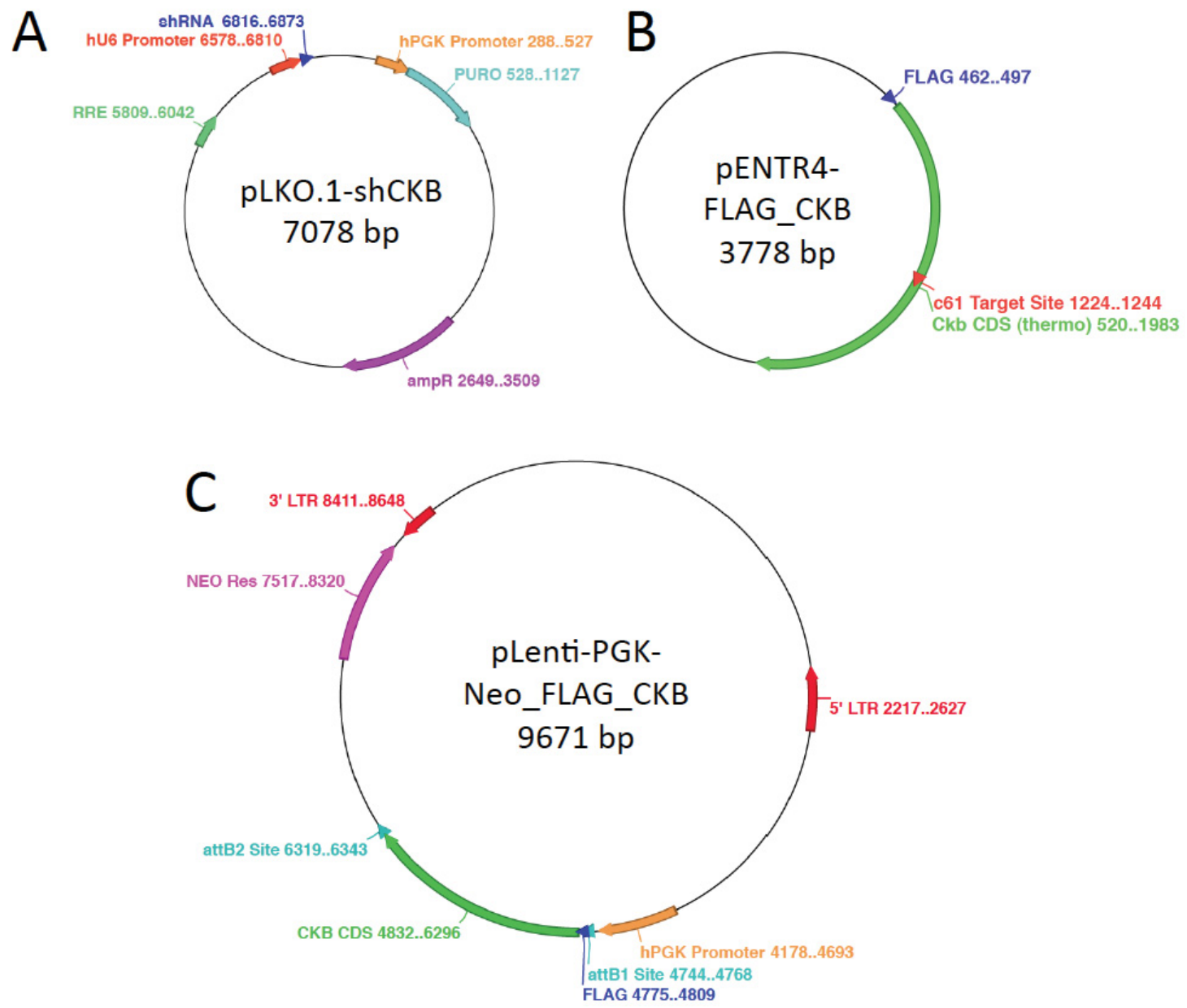

Figure 6-3. Vectors used to create $C k b$ cell line variants. 
conjugated to HRP or infrared dye, 680 or 800 . Antibody complexes were detected with chemiluminescence for complexes with HRP or imaged using a LiCor Odyssey infrared imaging system.

\section{RNA Extraction and Real Time PCR}

Total RNA was preparation and cDNA quantification were performed as described in Chapter 3.

\section{Invasion Assay}

Invasion assays were preformed as in Chapter 3.

\section{Cell Proliferation Assay}

MTECs were grown in normoxic culture (ambient air; 5\% $\mathrm{CO}_{2}$ ) or hypoxic culture $\left(0.5 \% \mathrm{O}_{2}, 5 \% \mathrm{CO}_{2}\right)$ in medium buffered with $25 \mathrm{mM}$ 4-(2-hydroxyethyl)-1piperazineethanesulfonic acid (HEPES). On the day before enumeration, 5,000 cells/ 100 $\mu \mathrm{L}$ were plated into 96 -well plates in normal growth medium ( $2 \%$ FBS medium). The medium was changed 24 hours post-plating, which coincides with the 0 time point $(t=0)$ and the first cell harvest for cell number analysis, but medium was not replenished for the duration of the experiment. All cells were plated in triplicate per genotype/oxygen tension/time point. For each time point, $10 \mu \mathrm{L}$ of WST-1 (Chemicon, 2210) reagent was added to cells and incubated for 2 hours at $37^{\circ} \mathrm{C}$ prior to measuring absorbance on a microplate reader. An absorbance of $440 \mathrm{~nm}$ was used for all samples, with a reference wavelength of $600 \mathrm{~nm}$. Background absorbance detected in blank wells was subtracted from sample wells and proliferation was measured as change in absorbance over time (in days).

\section{ATP Assay}

Intracellular ATP was measured from cultured MTECs following the protocols provided with the high sensitivity ATP bioluminescence assay kit (Roche, 11699709001). Briefly, MTECs were grown to $80 \%$ confluence prior to incubation in normoxic culture (ambient air; $\left.5 \% \mathrm{CO}_{2}\right)$ or hypoxic culture $\left(0.5 \% \mathrm{O}_{2}, 5 \% \mathrm{CO}_{2}\right)$ for 24 hours. All cells were plated in triplicate per genotype/oxygen tension. At 24 hours cells were harvested by scraping from the plate with a cell lifter and transferred into a $1.5 \mathrm{~mL}$ microcentrifuge tube. Samples were pelleted at 1,000 $\mathrm{g}$ for 10 minutes prior to lysis using the lysis reagent provided in the kit plus a protease and phosphatase cocktail inhibitor. Luciferase reagent was added 1 second prior to a 10 second integrated reading on a single-tube luminometer. A blank (reagent only) reading was obtained and subtracted from all sample readings. For the ATP standard curve, serial dilutions were made from the kit's ATP standard ranging 
from $10^{-5}$ to $10^{-12} \mathrm{M}$. Bioluminescence versus molarity was plotted on a log-log scale and used to calculate intracellular ATP concentrations in cell samples. DNA concentrations for each individual sample were measured using a Qubit ${ }^{\mathbb{B}}$ fluorometer (Invitrogen), and all ATP concentrations were normalized to total DNA for each individual sample.

\section{Tumor Transplant}

Tumor transplants were performed as described in Chapter 3. Briefly, MTECs dissociated into single cells with $0.05 \%$ trypsin-EDTA were counted using a hemacytometer and diluted 1:1 (vol:vol) with growth factor reduced Matrigel-Hank's balanced salt solution (HBSS) at a density of 50,000 cells per $10 \mu$ l. Cells were kept on ice until injection into the right inguinal mammary fat pads $(10 \mu \mathrm{l})$ of 3 -week old female $\mathrm{FVB} / \mathrm{Nj}$ recipients (The Jackson Laboratory) using a 26-gauge PT2 needle mounted on a Hamilton syringe, followed by clearing of the endogenous epithelium. Recipients were palpated one or two times per week, and outgrowths were measured with digital calipers to calculate tumor volume as described previously in Chapter 2 .

\section{Tail Vein and Cyclocreatine Experiments}

For studying of late stage metastatic events, PyMT EV, shc59 and shc61 $C k b$ KD single cells were injected directly into the circulation via the tail vein at a density of $1 \times 10^{6}$ cells per $100 \mu$ l. Mice were sacrificed after 21 days, and lungs were harvested for evaluation of metastases as described in Chapter 4.

For cyclocreatine experiments, PyMT EV cells were injected into the tail vein as above, and cells allowed to seed the lungs for 24h. Mice were then treated daily with 1 $\mathrm{g} / \mathrm{kg}$ of cyclocreatine (cCr) or vehicle ( $0.9 \%$ saline) i.p. for 21 days; dosing was based on [262]. In an independent experiment, a third cohort was added wherein PyMT EV cell micrometastases were allowed to form for 7 days prior to initiation of cCr therapy, which was then administered for 14 days. On day 21 all mice were sacrificed for lung harvesting, formalin fixation overnight and $\mathrm{H} \& \mathrm{E}$ staining.

\section{Tissue Harvest, Histology and Immunostaining}

Tumors were harvested from anesthetized mice and flash-frozen in liquid nitrogen for preparation of RNA or protein, or they were fixed in $10 \%$ neutral buffered formalin (NBF) for 6 hours at room temperature for histological staining (H \& E) and immunostaining. Paraffin-embedded sections (5 to $7 \mu \mathrm{m}$ ) were immunostained after antigen retrieval $(1 \times$ citrate buffer $)$ using a rabbit monoclonal antibody to $\mathrm{Ckb}$ (Epitomics 2830), followed by development with a VECTASTAIN Elite ABC Kit and ImmPACT DAB (diaminobenzidine) substrate (Vector Laboratories). Lungs were inflated with $10 \%$ NBF and fixed in NBF overnight. Paraffin-embedded sections $(7 \mu \mathrm{m})$ representing every $100 \mu \mathrm{m}$ of lung tissue were obtained for each paraffin block, and all 
sections were stained with $\mathrm{H} \& \mathrm{E}$. The individual $\mathrm{H} \&$ E-stained section containing the highest number of metastases per recipient, as evaluated by counting each slide under a light microscope at $\times 50$ original magnification, was used to determine the mean number of metastases per genotype.

\section{Chromatin Immunoprecipitation}

The promoter of $C k b$ was scanned for the presence of putative HIF response elements (HREs) using Transcription Factor Matrix (TFM) Explorer [266]. A 2500 bp sequence $(-2000$ to +500$)$ was scanned for the consensus HRE sequence using weight matrices available from JASPER and TRANSFAC. PyMT Hifla WT and KO cells were cultured at $21 \%$ (normoxia) or $0.5 \% \mathrm{O}_{2}$ (hypoxia) for 6 hours, and ChIP performed using primary antibodies against Histone H3 (AbCam 1791), HIF-1 $\alpha$ (Santa Cruz sc-10790X), or rabbit IgG, as the non-specific control. Primers were designed to flank the -1800 HRE, 5'-GTGCCTCCTGTGCACTTTTT-3' and 5'-AGCCTAGCTTCAGAGAACTAATGG3', the -1300 HRE, 5'-CAGGGCTGTTCTGGACTCTC-3' and 5'-

CCTCACAAGTFCTGGGATTA-3', and a region in a $C k b$ intron with no identified HRE, 5'-CCACAACAGCACATGACTGG-3' and 5'-GGCCTTAGATCACACCCAAG3'. Quantitative RT-PCR was performed on the isolated DNA. Site fold-enrichment was calculated as the fold change in HIF- $1 \alpha$ binding over the IgG control after normalization to the input DNA.

\section{Bioinformatics Analysis}

The level of $C K B$ mRNA expression in clinical breast tumor subtypes was derived from the TCGA data portal (http://cancergenome.nih.gov/). Level three normalized data were used for mRNA expression, and samples ( $\mathrm{n}=804$ total tumors) were stratified by tumor subtype based on the PAM50 method. Plots were generated using Prism 5.0 (GraphPad Software, Inc.) and $p$-values (ANOVA with Bonferroni correction) $\leq 0.05$ were considered significant. As the current TCGA data set provides limited survival data, survival analysis was also conducted using data derived from [267]. Normalized $\log$-ratio expression values for $C K B$ were divided into 3 equal tertiles: 96 tumors with the highest $C K B$ level (>499.7), 95 tumors with intermediate levels of $C K B$ (between 315.5 and 499.2) and 95 tumors with lowest $C K B$ levels $(<309.6)$. For the data analyzing $C K B$ together with HIFIA, 33 tumors expressed high levels of both HIFIA and $C K B, 37$ tumors low levels of both genes, and 32 tumors expressed intermediate levels of both genes. The y-axis (probability) is defined as the frequency of survival events. Data were analyzed using Prism 5.0 (Graph Pad San Diego, CA). 


\section{Results}

\section{Ckb Expression Is Decreased in Hifla KO Cells and Tumors}

PyMT MTEC Hifla WT or KO cells were exposed to $21 \% \mathrm{O}_{2}$ (normoxia) for 24 hours or $0.5 \% \mathrm{O}_{2}$ (hypoxia) for 6 or 24 hours. In contrast to expectations, hypoxia did not induce CKB protein expression as analyzed by western blot (Figure 6-4A). However, there was a dramatic decrease in CKB protein in either normoxic or hypoxic Hifla KO cells. This same pattern was observed for $C k b$ mRNA levels; there was a significant decrease in $C k b$ expression in the Hifla $\mathrm{KO}$ cells at both normoxia and hypoxia (Figure 6-4B). Cell extracts and RNA were extracted from whole tumor samples. The reduction of CKB protein between Hifla WT and KO end-stage tumors was modest with a $37 \%$ decrease in CKB protein expression in Hifla KO tumors (analyzed by Odyssey infrared western blot, Figure 6-4C). However, similar to cultured cells, $C k b$ mRNA expression showed a significant decrease in Hifla KO tumors as compared to WT. The discrepancy between the protein and mRNA results could be due to the fact that mRNA levels were first normalized to the epithelial cell marker, cytokeratin 18 (Krt18), to account for epithelial cell content in whole tumor samples, whereas the western blot was not normalized for cytokeratin expression. Therefore, we propose that the modest difference in $\mathrm{CKB}$ expression observed in the tumor samples could be due to CKB expression derived from infiltrating stromal cells in the whole tumor sample.

Experiments are in progress to co-stain WT and KO tumor sections with antibodies to CKB and to either cytokeratin 18, or to CD68, a marker of macrophages, or to CD45, a pan-leukocyte marker.

\section{Knockdown of $C k b$ Reduces Tumor Cell Invasion in vitro}

To assess the effect of loss of $C k b$ expression in vitro, Hifla WT cells were transfected with 3 independent pLKO.1 shRNA vectors targeting $C k b$. Of these 3 plasmids, two shRNA sequences produced $>75 \%$ knockdown at both the protein and mRNA level (Figure 6-5, shc59 and shc61) and these were then used to produce lentivirus particles to transduce PyMT HIF-1 $\alpha$ WT cells. Each stably transduced cell line produced $>80 \%$ reduction of $\mathrm{Ckb}$ protein and mRNA as compared to an empty vector (EV) control (Figure 6-6A and B). Next, cell proliferation was analyzed by the WST-1 assay over time in cells cultured at either normoxia or hypoxia. Neither shc59 nor shc61 produced any significant change in 2-D growth rate as compared to EV control cells cultured at normoxia or hypoxia (Figure 6-6C). However, when assayed for invasion potential in a trans-well assay, there was a significant reduction in the ability of each knockdown cell line to invade through a Matrigel layer as compared to EV cells (Figure 6-6D). This reduction was even more pronounced at hypoxia. 

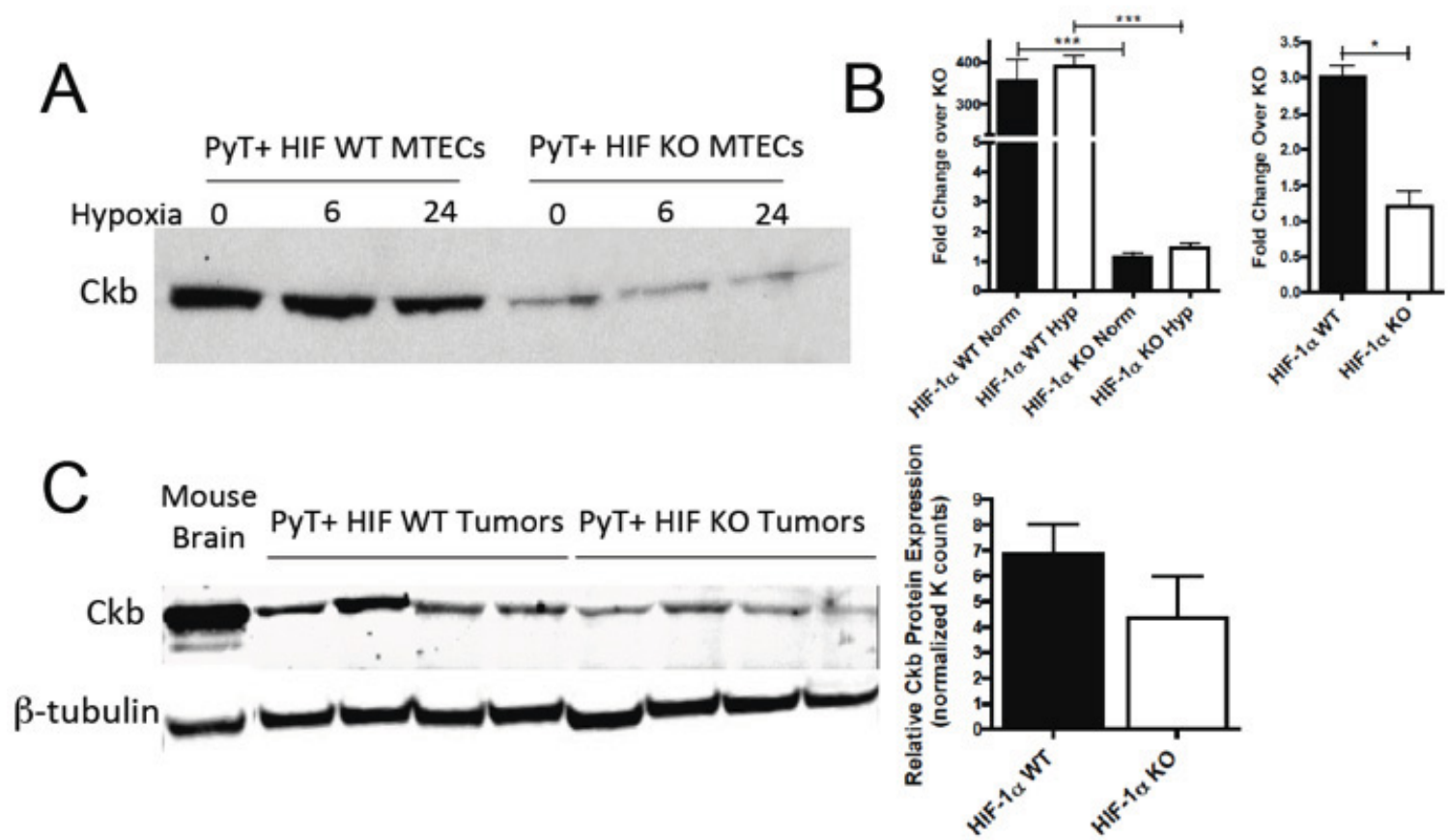

Figure 6-4. $\quad C k b$ expression in PyMT Hif1a WT and KO cells and end-stage tumors.

A. PyMT MTECs grown to $80 \%$ confluence were subjected to hypoxia culture for the indicated number of hours for up to $24 \mathrm{~h}$, or cells were continuously cultured at normoxia, such that the $\mathrm{t}=0$ sample was harvested on the same day as the $\mathrm{t}=24 \mathrm{~h}$ hypoxic sample.

WCE were resolved on 4-12\% Tris-Base gels and blotted to PVDF membrane and blotted for CKB. B. The mean fold-change \pm S.E.M. in $C k b$ mRNA gene expression observed in WT and KO cells and end-stage WT and KO tumors as determined by quantitative realtime PCR ( $\mathrm{n}=3$ tumors/genotype). C. Western blotting for CKB comparing four independent tumors $\left(500-750 \mathrm{~mm}^{3}\right.$ ) per genotype (mouse brain WCE was used as a positive control). Extracts were resolved on 4-12\% Tris-Base gels and blotted to PVDFFluorescent membrane which was divided horizontally at approximately $50 \mathrm{kDa}$. The top half of the blot was blotted for CKB and the lower portion was blotted for $\beta$-tubulin (loading control). Blots were imaged on a LiCor Odyssey infrared imaging system and $\mathrm{CKB}$ expression was determined in WT versus KO tumors after normalization to $\beta$ tubulin levels (right panel). The difference in CKB expression trended toward significance $(p=0.13)$, but additional samples $(n>4)$ will need to be analyzed to increase statistical power. 

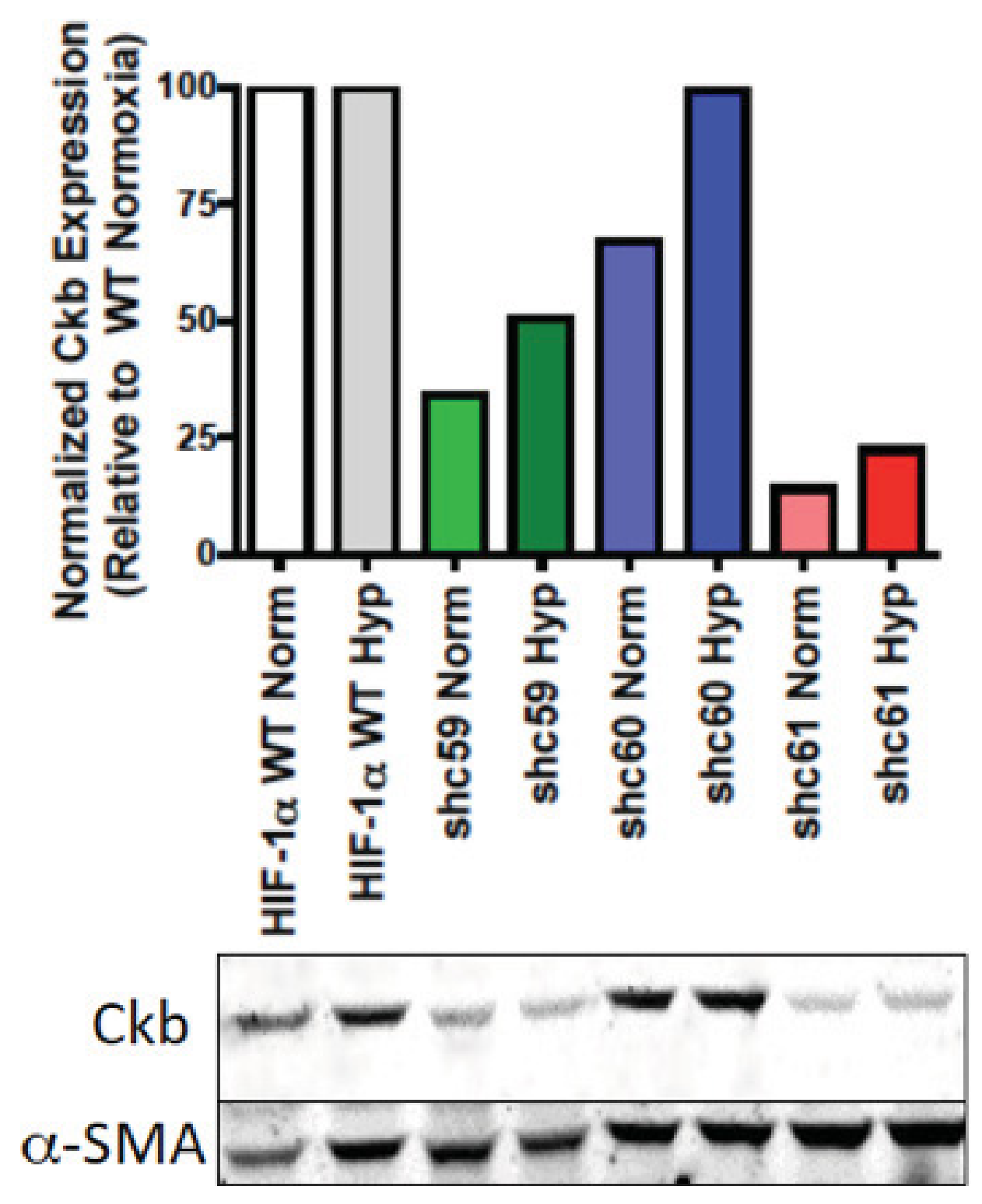

Figure 6-5. Evaluation of 3 independent $C k b$ shRNA vectors to efficiently knockdown CKB in cells transfected with pLKO.1 viral vector DNA.

PyMT HIF-1 $\alpha$ WT cells were transfected with $4 \mu \mathrm{g}$ of pLKO. 1 shRNA vector DNA by nucleofection. Cells were subjected to puromycin selection 24 hours after transfection and resulting colonies were pooled to create stable knockdown cell lines. WCE were resolved on $4-12 \%$ Tris-Base gels and blotted to a PVDF-FL membrane which was blotted for CKB and $\alpha$-SMA (loading control). Blots were imaged on an Odyssey infrared imaging system and $\mathrm{CKB}$ expression was determined in WT versus KD cells after normalization to $\alpha$-SMA levels. 
Figure 6-6. Effect of $\boldsymbol{C k b}$ knockdown upon growth and invasion.

A. PyMT MTECs grown to $80 \%$ confluence were subjected to normoxic or hypoxia culture for 6 hours. WCE were resolved on 4-12\% Tris-Base gels and blotted to PVDF membrane which was divided horizontally at approximately $50 \mathrm{kDa}$. The top half of the blot was blotted for CKB and the lower portion was blotted for $\beta$-tubulin (loading control) to avoid the need to strip and reprobe the blot. B. The mean fold-change \pm S.E.M. in $C k b$ gene expression observed relative to EV as determined by qRT-PCR. C. Growth curve of MTECs cultured at normoxia or hypoxia in growth medium supplemented with $2 \%$ FBS. For both conditions, the mean absorbance \pm S.E.M. is presented, calculated as an average of the mean cell number observed per replicates per time point, as observed in two replicate experiments. All data was analyzed by two-way ANOVA and was not significant at any time point $(p>0.05)$. D. The mean fold-change in invasion was normalized to the invasion index observed for EV cells cultured at normoxia $(\mathrm{FC}=1.0)$. Data represent the mean fold change in invasion observed in two independent experiments. All columns were compared to each other using one-way ANOVA analysis with a Bonferroni post-test, ${ }^{*} p<0.05, * * * p<0.001$. E. MTECs were plated in triplicate and grown to $80 \%$ confluence prior to being subjected to 24 hours normoxic or hypoxic culture. Cells were harvested and immediately lysed for analysis of intracellular ATP. ATP concentrations were determined from a standard curve of serial dilutions of ATP protein and normalized to total DNA. The mean ATP concentration per ng of DNA \pm S.E.M. is presented calculated as an average of the 3 technical replicates per cell type. Data was analyzed by one-way ANOVA with a Bonferroni correction posttest, $* p<0.05, * * * p<0.001$ and represents the mean from two independent biological replicates. 

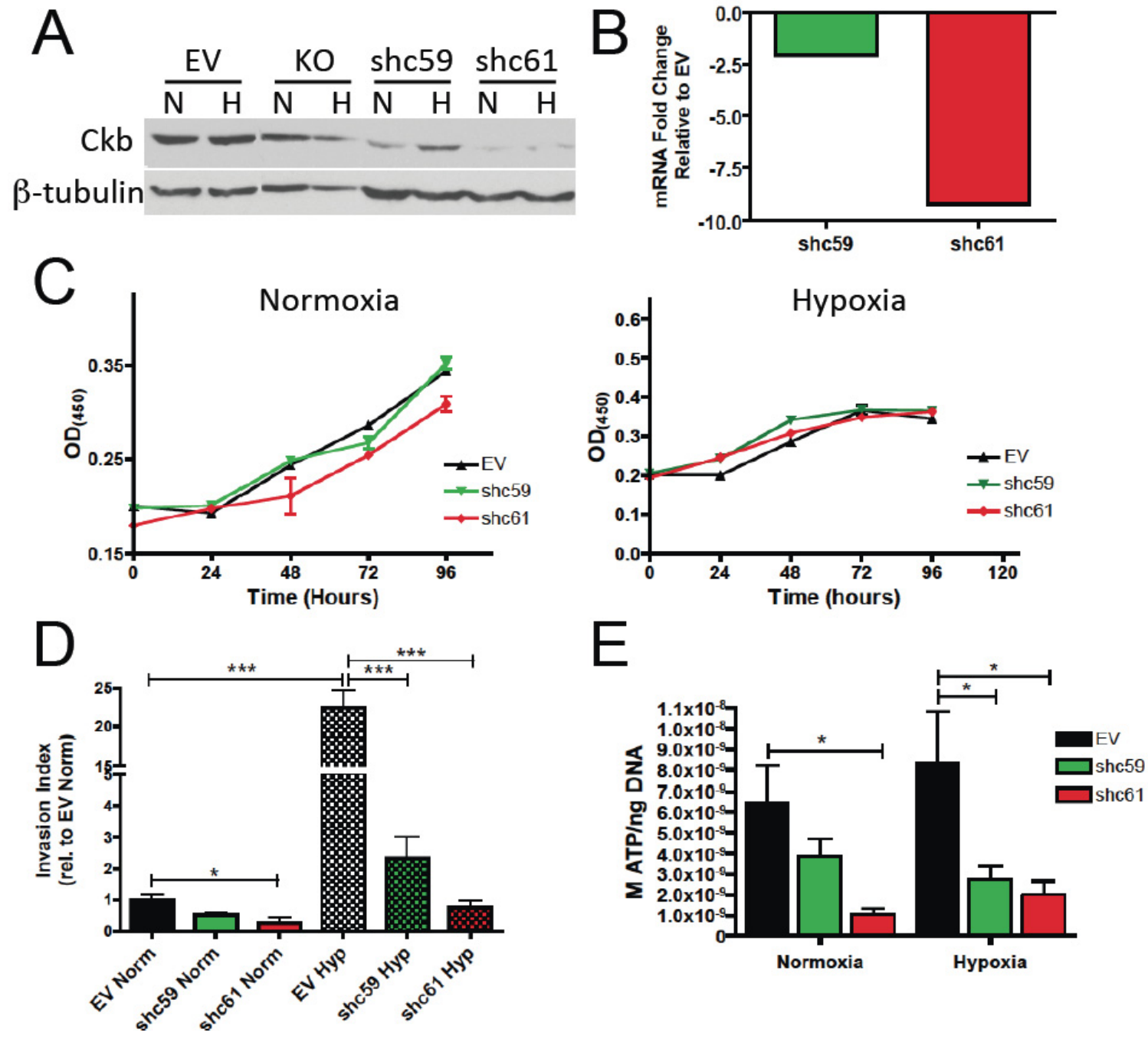


\section{Loss of $C k b$ Significantly Delays Tumor Formation and Inhibits Lung Metastases in vivo}

To study the effects of $C k b$ knockdown in vivo, we first chose to compare primary tumor growth rate. HIF-1 $\alpha$ WT (EV), HIF-1 $\alpha$ KO, shc59 and shc61 cells were transplanted into the inguinal fat pad of FVB mice at a density of 50,000 cells. Tumors were palpated at least once per week over time and measured to calculate tumor volume. As shown previously [127], Hifla KO cells exhibited a significant delay in tumor onset and decreased tumor volume maintained over time. A similar pattern of growth and size was observed in both $C k b$ knockdown cell lines (Figure 6-7A). At day 35 post transplant, the tumor volume of EV tumors was $\geq 1000 \mathrm{~mm}^{3}$, whereas all other genotypes had reached a maximum mean volume of only $250 \mathrm{~mm}^{3}(p<0.001)$. At endpoint there was also a significant difference in tumor wet weight among EV, shc59, and shc61 (Figure 6-7B, $p<0.001$ ). To investigate if loss of $C k b$ blocked or delayed metastasis from the primary tumor, in a small independent pilot experiment all tumors were resected at a volume of $\sim 750 \mathrm{~mm}^{3}$, and animals were housed until moribund due to lung metastases. Recipients of shCkb tumors survived $>2 x$ longer than recipients of EV cells ( 7.5 wks vs. 3.5 wks, hazard ratio 19.66 by log-rank test, Figure 6-7C). To explore if $C k b$ knockdown also blocked late-state metastatic events, a tail vein assay was utilized. One million cells were injected directly into the circulation and mice were harvested 21 days later. The lungs of all FVB recipient mice injected with WT or EV cells were filled with large metastases, whereas no surface mets were observed for the shc61 cells and a maximum of 10 metastases were observed for shc59 cells (Figure 6-8).

\section{Inhibition of Ckb with Cyclocreatine Blocks Lung Metastasis}

Next, PyMT EV cells were injected into the tail vein, and allowed to seed the lungs for $24 \mathrm{~h}$. After 24 hours mice began receiving daily treatment with cyclocreatine (cCr), a phosphocreatine analog, at a dosing of $1 \mathrm{~g} / \mathrm{kg}$ of $\mathrm{cCr}$ or vehicle $(0.9 \%$ saline $)$ i.p. for 21 days. Whereas surfaces of lungs from vehicle-treated mice were filled with metastases, very few metastases were visible in the $\mathrm{cCr}$ cohort (Table 6-1). These results were replicated in an independent experiment, in which a third a cohort was added; micrometastases were allowed to form for 7 days prior to initiation of $\mathrm{cCr}$ therapy for 14 days. In this cohort, $2 / 5$ mice developed $>50$ surface metastases, whereas all $(5 / 5)$ vehicle-treated mice developed $>50$ metastases and $1 / 5$ mice treated from day 1 with $\mathrm{cCr}$ developed $>50$ metastases (Figure 6-9, Table 6-2, $\chi^{2}<0.05$ ).

\section{CKB Expression Correlates with HIF1A mRNA Expression and with Decreased Relapse Free Survival in Patients with Breast Cancer}

To gain insight into the clinical relevance of $C K B$ gene expression in breast cancer, we examined its expression in a collection of primary tumor samples. By evaluating the TCGA breast cancer data set, we found that $C K B$ mRNA levels are significantly upregulated in HER2-enriched breast cancers compared to basal-like or 


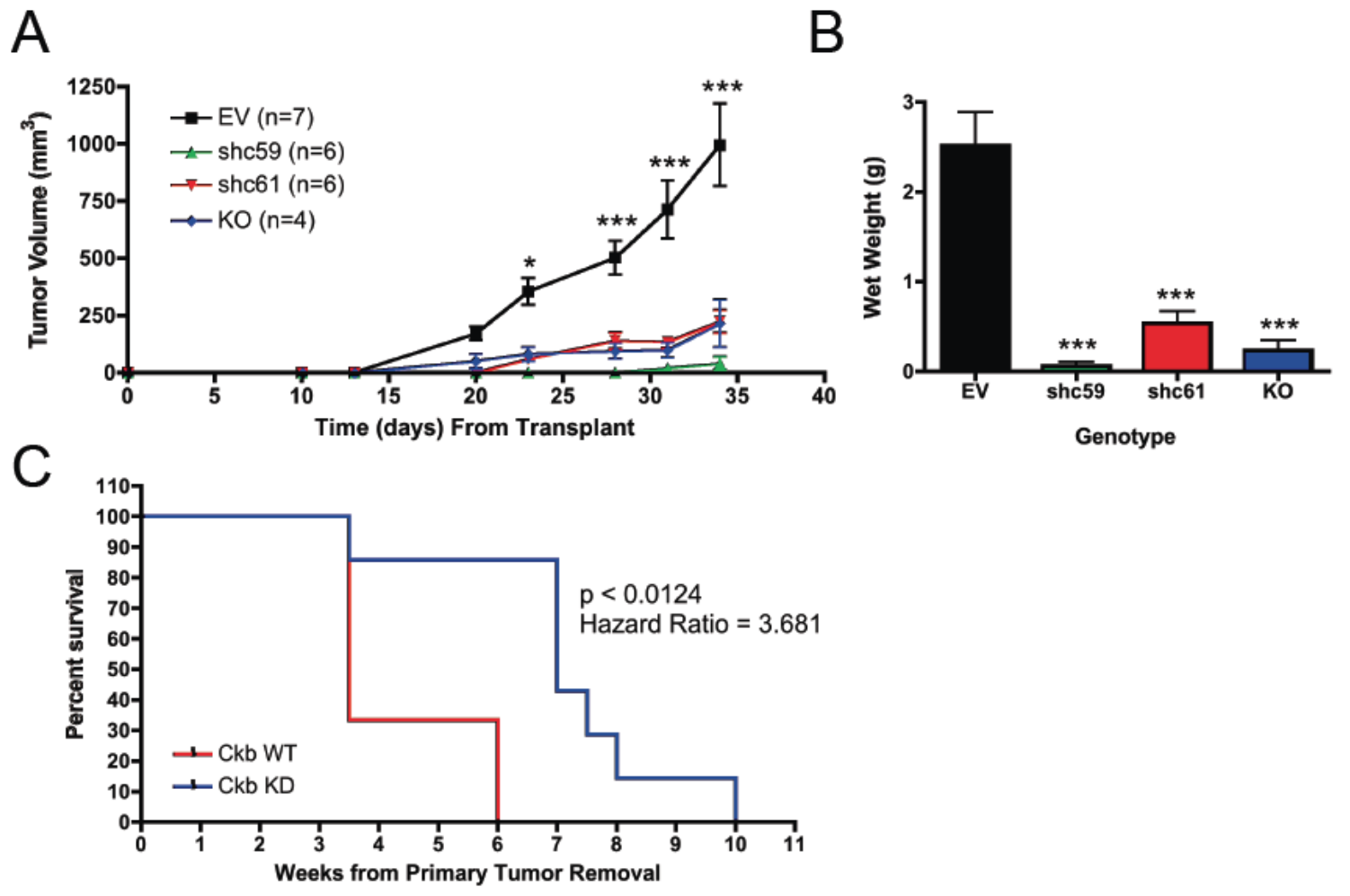

Figure 6-7. $\quad C k b$ knockdown decreases primary tumor growth and end point tumor wet weight and increases survival time from lung metastases.

A. The growth rate of PyMT HIF-1 $\alpha$ WT (EV), KO, shc59 and shc61 tumors at 50,000 cells input $\left({ }^{*} p<0.05\right.$ at day 23 and $* * * p<0.001$ from day 27 to end point, two-way ANOVA with Bonferroni correction). B. All tumors were harvested at day 34 to evaluate tumor weight $(* * * p<0.001$, ANOVA with Bonferroni correction). Data in A-B are representative of two independent experiments. C. The impact of $C k b$ expression in tumor cells on the survival of recipients following tumor resection. A pilot experiment of a subset of animals subjected to primary tumor resection and allowed to survive until moribund due to metastasis. The morbidity hazard ratio is 3.68 times higher when hosts bear Ckb WT tumors ( $\mathrm{n}=4$ mice/genotype, log-rank test, $p=0.0124$ ). 


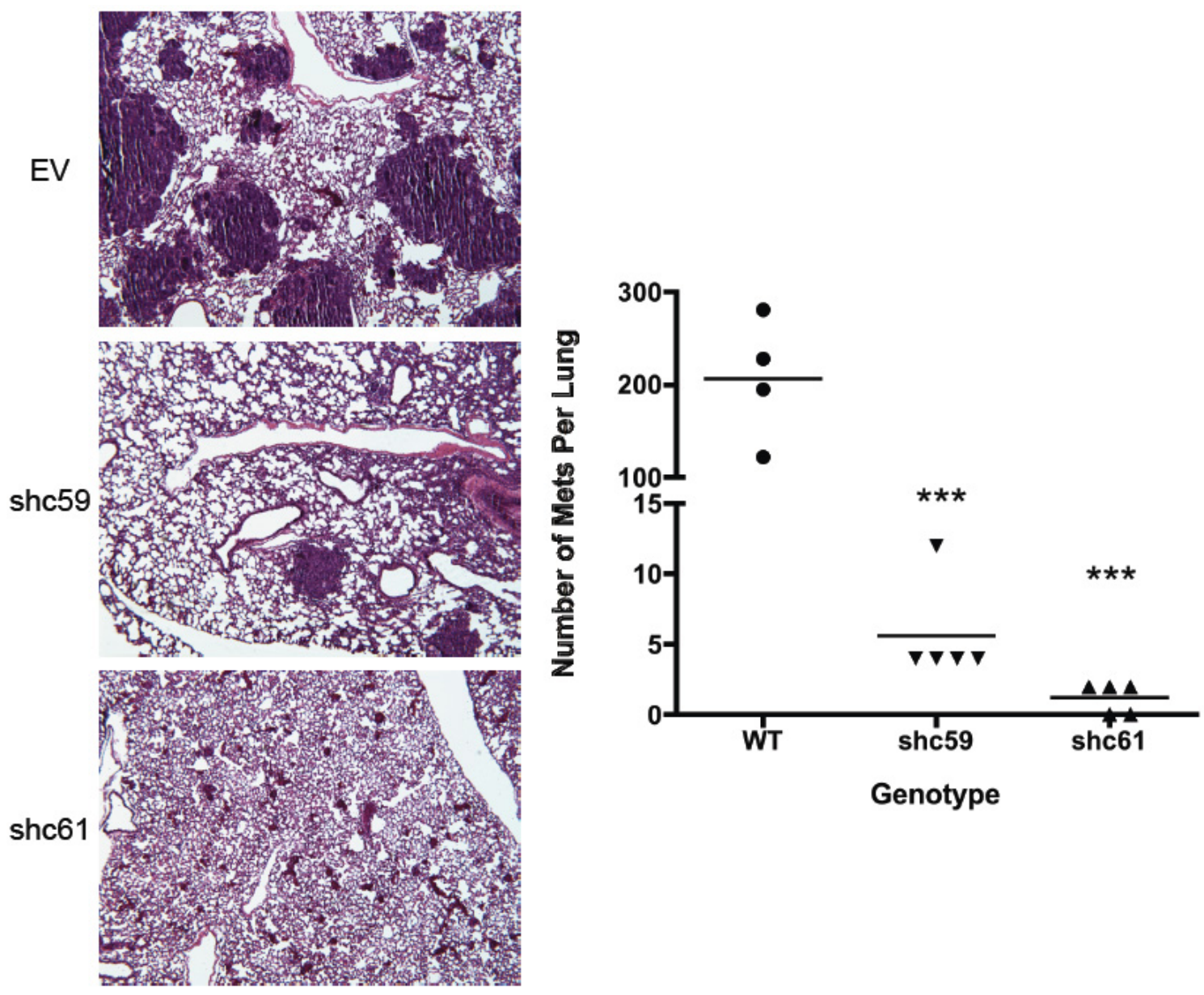

Figure 6-8. Knockdown of $\boldsymbol{C k b}$ decreases lung metastasis burden.

EV, shc59 or shc61 MTECs at a density of $1 \times 10^{6}$ cells/ $100 \mu \mathrm{L}$ were injected directly into pre-warmed tail veins of $\mathrm{FVB} / \mathrm{Nj}$ recipient mice. After 21 days mice were sacrificed and lungs harvested for metastasis evaluation. Images were captured at 40x magnification. The total number of surface metastases $(\mathrm{n}=5$ mice/genotype $* * * p<0.001)$ were counted under a dissecting scope. $\mathrm{H} \& \mathrm{E}$ analysis revealed metastasis size was reduced in response to $C k b$ knockdown. This experiment is representative of 4 biological experiments with a grand total of $n>40$ mice/genotype. 
Table 6-1. Summary of surface lung metastases in mice treated with saline versus cyclocreatine for 21 days.

\begin{tabular}{lcc}
\hline $\begin{array}{c}\text { Metastatic } \\
\text { Event }\end{array}$ & Saline & Cyclocreatine \\
\hline No Mets & $1 / 8$ & $5 / 7$ \\
$<50$ Mets & $2 / 8$ & $2 / 7$ \\
$>100$ Mets & $5 / 8$ & $0 / 7$ \\
\hline
\end{tabular}

$\chi^{2}=7.634, p=0.022$ 


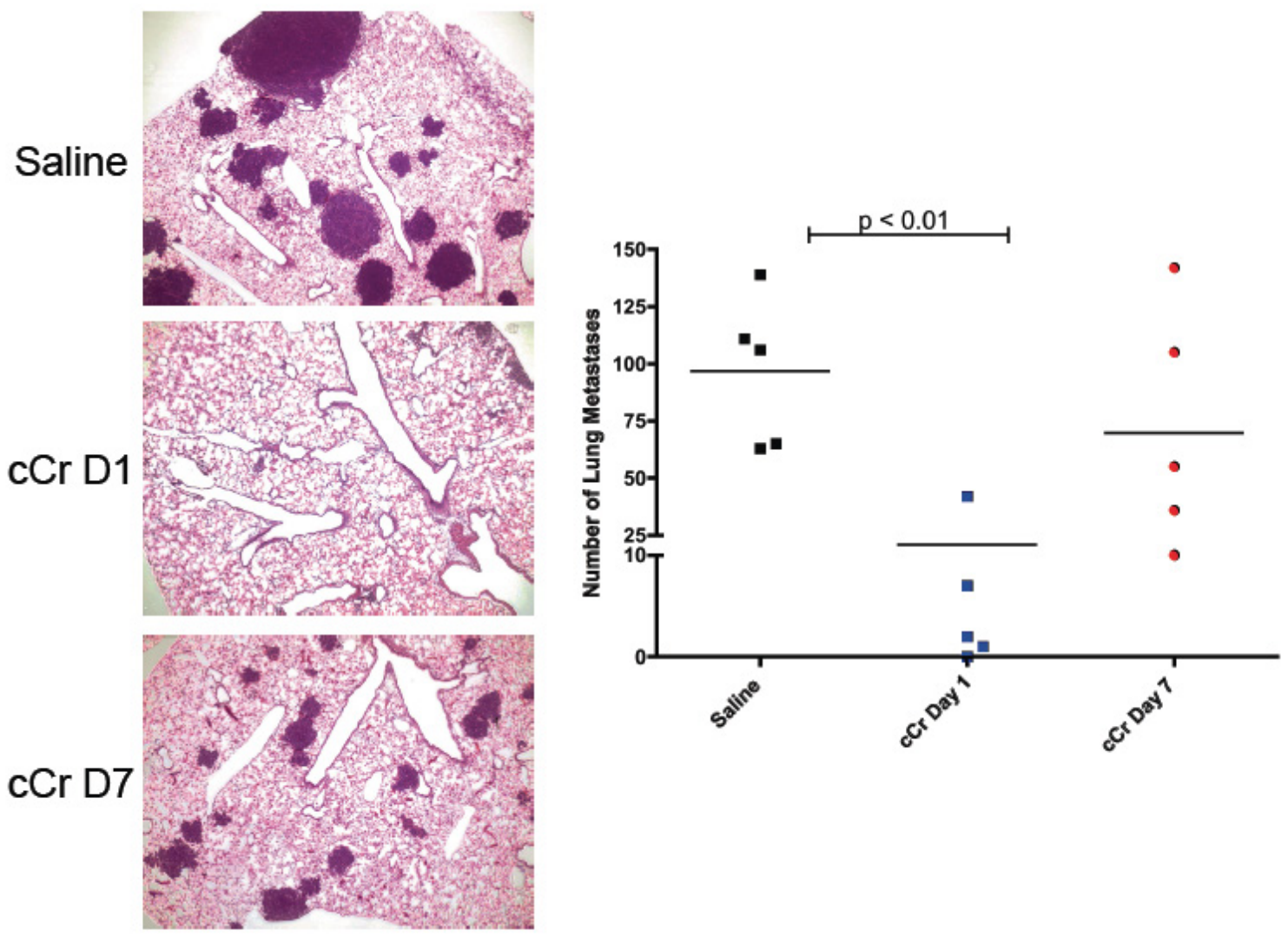

Figure 6-9. Inhibition of $\mathrm{Ckb}$ by cyclocreatine (cCr) treatment blocks the formation of lung metastases.

EV MTECs were injected directly into pre-warmed tail veins of $\mathrm{FVB} / \mathrm{Nj}$ recipient mice at a density of $1 \times 10^{6}$ cells $/ 100 \mu \mathrm{L}$. Mice were divided into three groups and after 24 hours, one group $(n=5)$ began receiving daily IP injections of saline (Saline) and another $(n=5)$ began receiving daily IP injections of $1 \mathrm{~g} / \mathrm{kg}$ cyclocreatine (cCr D1). After 7 days, when micrometastases have formed, the third group of mice $(n=5)$ began receiving daily injections of $1 \mathrm{~g} / \mathrm{kg}$ cyclocreatine (cCr D7). Three weeks (21 days) after initial cell injection, all mice were sacrificed and lungs harvested for metastasis evaluation. Images were captured at 40x magnification. Each lung harvested was analyzed for total number of surface metastases ( $n=5$ mice/genotype) and H\&E sections were also evaluated to compare metastasis size. This experiment represents one pilot experiment of 2 different starting points. However, the saline versus day 1 start point data in this second experiment, replicate the results shown in Figure 6-8. 
Table 6-2. Summary of surface lung metastases in mice treated with saline versus cyclocreatine for 21 days (day 1 start) or cyclocreatine for 14 days (day 7 start).

\begin{tabular}{cccc}
\hline $\begin{array}{c}\text { Metastatic } \\
\text { Event }\end{array}$ & Saline & Day 1 Start & Day 7 Start \\
\hline No Mets & $0 / 5$ & $3 / 5$ & $0 / 5$ \\
$<10$ Mets & $0 / 5$ & $2 / 5$ & $2 / 5$ \\
$11-50$ Met & $0 / 5$ & $0 / 5$ & $2 / 5$ \\
$>50$ Mets & $5 / 5$ & $0 / 5$ & $1 / 5$ \\
\hline
\end{tabular}

$\chi^{2}=17.43, p=0.0078$ 
luminal breast cancers (Figure 6-10A). As expected, based on data from the PyMT model, increased levels of $C K B$ mRNA also correlated with increased levels of HIFIA mRNA across all subtypes (Figure 6-10B). We also investigated how $C K B$ expression related to survival in breast cancer patient. Patients with high $C K B$ expression had significantly shorter times for recurrence free survival as compared to those with low or intermediate levels of $C K B$ (Figure 6-10C). Relapse time was further decreased in patients who had both high levels of HIFIA and $C K B$ (Figure 6-10D).

\section{Is $C k b$ a Direct HIF-1 $\alpha$ Target?}

Since $C k b$ expression is dependent upon HIF-1 $\alpha$ expression in PyMT WT and $\mathrm{KO}$ cells and tumors, and because $C K B$ mRNA expression correlates with $H I F 1 A$ mRNA expression in primary breast cancer samples, we sought to determine if $C k b$ is directly regulated by HIF-1 $\alpha$. Upon examination of the mouse $C k b$ promoter, we identified two potential hypoxia response elements (HREs) within $2 \mathrm{~kb}$ of the transcriptional start site (0) (Figure 6-11, top panel). To examine HIF- $\alpha$ recruitment to the $C k b$ promoter, we performed chromatin-immunoprecipitation (ChIP) assays with PyMT HIF-1 $\alpha$ WT and KO cells cultured at hypoxia for 6 hours. We observed that HIF-1 $\alpha$ did not appear to be recruited to either HRE (Figure 6-11, bottom panel). HIF-1 $\alpha$ recruitment to the VEGF promoter was included as a positive control, but also did not appear to recruit HIF-1 $\alpha$ to its promoter region, suggesting a technical problem with the ChIP procedure. Previous control experiments using a different ChIP grade antibody (Abcam 2185), produced a site fold enrichment of $>5$ for HIF- $1 \alpha$ binding at the VEFG HRE, whereas in the experiment presented here (using the Santa Cruz antibody), there was a fold enrichment of $<1$. This suggests that the Abcam antibody may have a higher binding affinity to mouse HIF-1 $\alpha$ than the Santa Cruz antibody, while the Santa Cruz antibody binds well to human HIF-1 $\alpha$ (Figure 4-3). As a functional correlate of transcriptional activity associated with the VEFG HRE, we assessed the recruitment of Histone $\mathrm{H} 3$ to this region and observed robust recruitment of this enzyme following exposure to hypoxia. These data suggest that HIF-containing transcriptional complexes present at the VEFG HRE, further suggesting there may be a problem with the HIF-1 $\alpha$ antibody used for this experiment being recruited. The first intron in the $C k b$ promoter served as a negative control (Figure 6-11). As this experiment served only as a preliminary examination into HIF-1 $\alpha$-dependent regulation of $\mathrm{Ckb}$, and the results were inconclusive as judged by the failure of the positive control, additional experiments are needed to ascertain the exact mechanism of HIF-1 $\alpha$ regulation of $\mathrm{Ckb}$.

\section{Discussion}

Although multiple roles for HIF- $1 \alpha$ in mediating breast cancer phenotypes have been well described [68, 126, 127, 198, 268-271], there are still many HIF-1 $\alpha$-dependent genes that remain to be characterized for the role they play in tumor progression and metastasis. We have identified one such gene, creatine kinase brain isoform $(C k b)$ to be a 

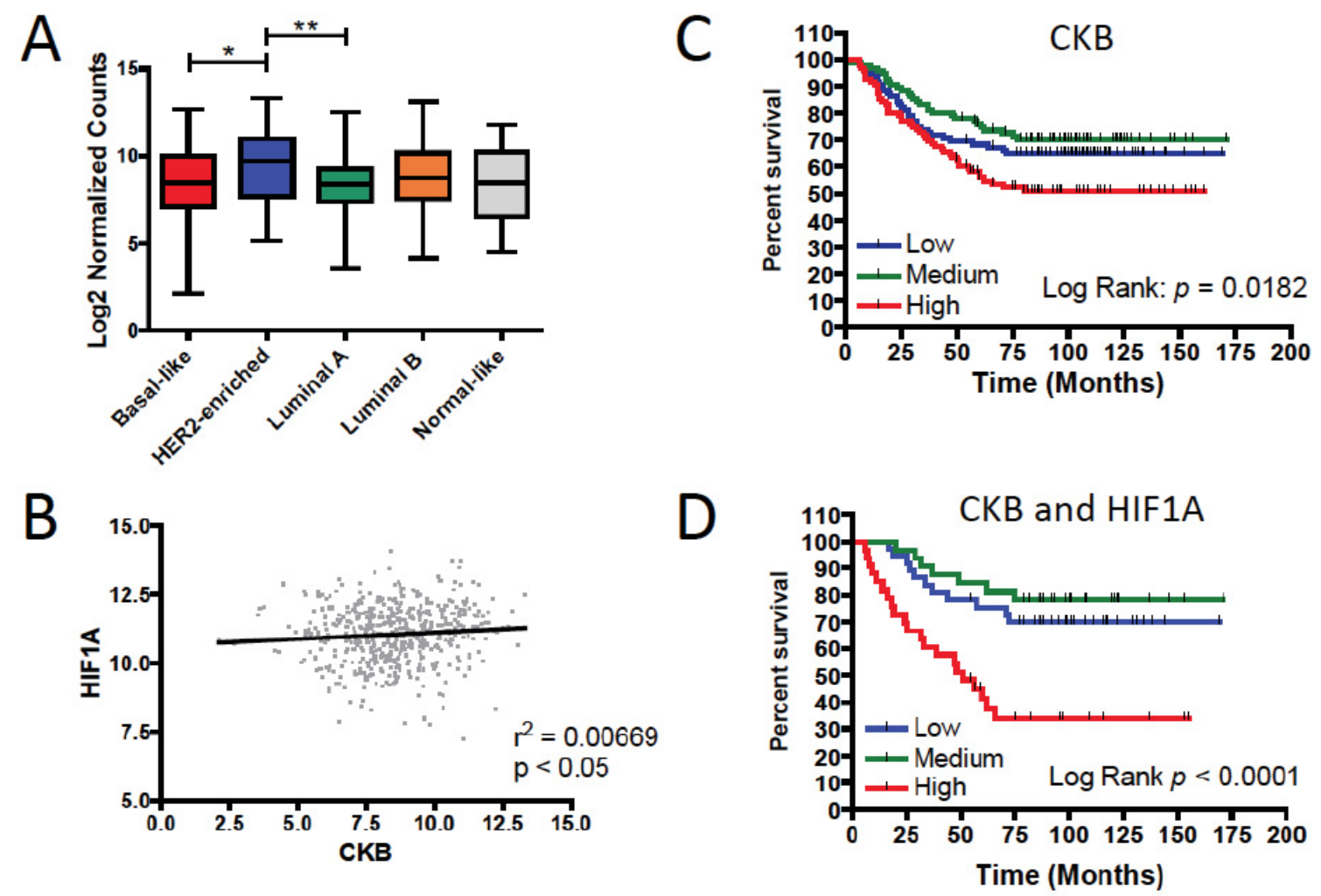

Figure 6-10. $C K B$ correlates with $H I F 1 A$ in primary breast cancer samples. A. $C K B$ mRNA levels were compared using the Cancer Genome Atlas (TCGA) dataset across breast cancer subtypes based on PAM50 classification. B. Normalized $C K B$ mRNA was compared to HIF1A mRNA for all samples to determine correlation of the two in the TCGA dataset $\left(\mathrm{r}^{2}=0.00669, p<0.05\right)$. C. Kaplan-Meier curves were derived from data analyzed from 286 primary human breast tumor specimens [267] for recurrence-free survival, stratified by high, intermediate (medium) and low $C K B$ expression. D. Stratification incorporating both $C K B$ and HIF $1 A$ expression levels. Significance was determined by Mantel-Cox Log-rank test. 

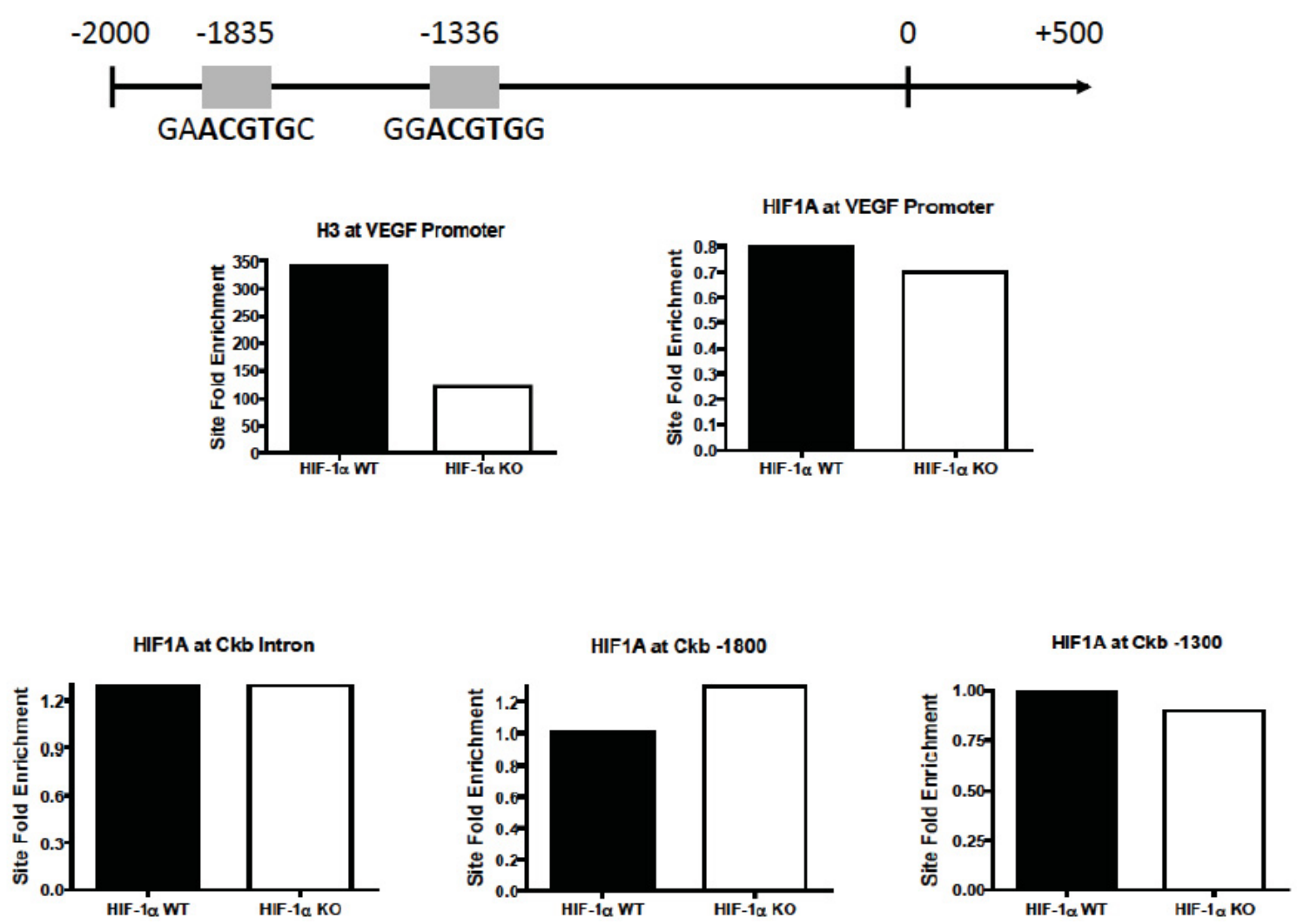

Figure 6-11. Preliminary ChIP analysis of the $\boldsymbol{C k b}$ promoter.

Top panel: schematic representation of HREs in the proximal $C k b$ promoter that were assessed for HIF-1 $\alpha$ recruitment. Lower panels: PyMT HIF-1 $\alpha$ WT and KO cells were cultured at hypoxia $\left(0.5 \% \mathrm{O}_{2}\right)$ for 6 hours. Formaldehyde-fixed lysates were subjected to ChIP with HIF-1 $\alpha$ or Histone $\mathrm{H} 3$ antibodies and qPCR was performed on the isolated DNA to determine HIF- $1 \alpha$ and H3 recruitment to the HREs. As controls, ChIP for HIF$1 \alpha$ was performed for the mouse $V e g f$ promoter or an intron within $C k b$ with no identified HRE sites. Data are derived from a pilot experiment and are representative of a single experiment. 
HIF-1 $\alpha$-dependent gene necessary for mammary tumor growth and lung metastasis in the PyMT model of breast cancer. We have demonstrated that HIF-1 $\alpha \mathrm{KO}$ cells and tumors express much lower levels of both $\mathrm{Ckb}$ protein and mRNA. Interestingly, while $\mathrm{Ckb}$ was highly HIF-1 $\alpha$ dependent in these experiments, it was not hypoxia-inducible. One explanation for this could be that since most breast cancers over express HIF-1 $\alpha$, no hypoxic stimulation is needed to induce expression of Ckb. Furthermore, as HIF expression can be induced independently of hypoxia by growth factor pathways such as EGF and HER2, the HIF-1 $\alpha$-dependent expression of Ckb may be through one of these pathways. Additionally, cell stressors such as low glucose, change in $\mathrm{pH}$ and theproduction of reactive oxygen species (ROS), all increase HIF-1 $\alpha$ expression and could up-regulate $\mathrm{Ckb}$ expression independent of hypoxia.

Analysis of Ckb levels in HIF-1 $\alpha$ WT and KO tumors showed a robust decrease in both mRNA and protein expression in KO MTECs. However, in tumors this difference was not as dramatic. A significant difference in mRNA expression was only observed after data was normalized to the epithelial cell maker, Krt18. In tumors the difference was not significant. However, this could be due to the fact that protein extracts were derived from whole tumor samples including both stromal and epithelial cells, and samples were not normalized to Krt18 expression for epithelial cell content only. Therefore, some of the $\mathrm{Ckb}$ expression observed in tumors may be from invading stromal cells. To identify if $\mathrm{Ckb}$ is highly expressed in invading stromal cells in $\mathrm{KO}$ tumors, whole tumors could be sorted for based on Krt18 expression to isolate epithelial cells from stromal cells and $\mathrm{Ckb}$ expression analyzed in these isolated cells. This could indicate if the increase in HIF-1 $\alpha$ $\mathrm{KO}$ tumor growth over time may be due to the increase in infiltrating stromal cells that express high levels of Ckb over time.

Knockdown of the $C \mathrm{~kb}$ gene produced decreased mammary tumor growth, similar to data derived from HIF-1 $\alpha$ KO cells. However, in contrast to HIF-1 $\alpha \mathrm{KO}$ cells, tail vein assays to model late state metastasis revealed a significant decrease in lung metastases produced either by knocking down $C k b$ or inhibiting its activity with the chemical inhibitor, cCr. Together these results suggest $\mathrm{Ckb}$ acts downstream of HIF-1 $\alpha$ to promote late stage metastatic breast cancer. This finding is particularly relevant to breast cancer subtypes that exhibit high constitutive expression of HIF-1 $\alpha$, including basal-like and HER2-enriched tumors which are both enriched for the hypoxic core signature $[92,93]$.

Initial ChIP assays to determine if $C k b$ is a direct HIF- $1 \alpha$ transcriptional target gene did not produce conclusive results. While the assay control for $\mathrm{H} 3$ binding to the $V e g f$ promoter was clear, no assays performed with the HIF-1 $\alpha$ antibody displayed enrichment for binding of HIF-1 to the $V e g f$ HRE or to the $C k b$ regulatory region $(-2000$ to $+500 \mathrm{bp}$ ). Prior control studies performed with a different HIF-1 $\alpha$ antibody (Abcam 2185) produced a site enrichment of at least 2-fold in HIF-1 $\alpha$ WT cells at the Vegf promoter, however in Figure 6-11, the Santa Cruz sc-10790X HIF-1 $\alpha$ antibody was used and produced less than a 1-fold site enrichment. These data suggest that the Santa Cruz antibody may not display a strong affinity for mouse HIF-1 $\alpha$. To confirm whether or not HIF- $1 \alpha$ can bind to the $C k b$ promoter, additional experiments need to be performed 
utilizing either a different HIF-1 $\alpha$ antibody or a different positive HIF-1 $\alpha$ control such as $P h d 3$, which was shown on our microarray data to be more HIF-1 $\alpha$ dependent than Vegf. Additionally, a human cell line that expresses high endogenous levels of CKB, such as MCF7 cells that have been knocked down for HIF-1 $\alpha$, HIF-2 $\alpha$, and DKD for both [26], could be tested, as there were HREs identified in the human CKB promoter as well. Of note, we have screened the MDA-MB-231 knockdown cells for Ckb expression and no detectable levels of $\mathrm{Ckb}$ protein were observed in any genotype, so these cells are not useful for CKB evaluation.

The commercial availability of the creatine kinase (CK) inhibitor, cyclocreatine (cCr) [272], provides an excellent chemical tool to ascertain the effects of loss of $\mathrm{Ckb}$ activity upon the formation of metastases, as an alternative to genetic manipulation. Blocking CK activity by daily treatments with cCr resulted in a significant decrease in the ability of Ckb expressing PyMT WT cells to form metastases in the lungs of recipient mice. Additionally, conversion of micrometastases to macrometastases was also blocked by $\mathrm{cCr}$ treatment, as was demonstrated by starting $\mathrm{cCr}$ treatment 7 days after cells were injected into mice. One caveat to these studies however, was the lack of additional dosing regimens. Our single dose of $1 \mathrm{~g} / \mathrm{kg}$ was based on data published from almost 20 years ago that investigated $\mathrm{cCr}$ effects on rat mammary adenocarcinomas [262]. There were no adverse side effects or obvious gross toxicities using the $1 \mathrm{~g} / \mathrm{kg}$ dose in FVB mice. However, this dose may not be the maximum effective dose. Studies are needed that determine the maximum tolerated dose tolerated by FVB mice bearing tumor cells, as well as more thorough cytotoxicity studies of mouse organs following treatment with escalating doses of $\mathrm{cCr}$ to determine if a higher dose could also be used to block metastases. Furthermore, no studies were completed to examine the effects of $\mathrm{cCr}$ on primary tumor growth, or if $\mathrm{cCr}$ could also block metastatic events following removal of the primary tumor. Both are future experiments I plan to complete.

Another caveat to the $\mathrm{cCr}$ experiments was the use of a single model, the PyMT model. A screen of CKB protein expression in a panel of the HCI patient derived xenograft tumors demonstrated that while all tumors express $\mathrm{CKB}$, a subset of TNBC tumors express higher levels of the protein, including the TNBC tumor HCI-10. As will be discussed in the next chapter, HCI-10 tumors metastasize to the axillary lymph node and because the tumor cells are labeled with Luc2, mice bearing HCI-10 tumor cells can easily be monitored for not only primary tumor growth, but also metastatic events. With this system we can evaluate two areas in which cCr therapy could be beneficial; 1) inhibiting tumor progression after the formation of a measurable tumor mass and 2) blocking the formation of macrometastases after surgical resection of the primary tumor. The second of these would be most useful in evaluating $\mathrm{cCr}$ as a clinically relevant therapy as most patients with breast cancer will undergo surgery to remove the primary mass, and are at a high risk of metastatic recurrence and death.

Furthermore, I found that $\mathrm{CKB}$ is a prognostic factor in breast cancer. High $C K B$ mRNA expression in primary human breast tumors is significantly correlated with a decrease in recurrence free survival, and survival further decreases when high $C K B$ expression is paired with high $H I F 1 A$ expression. This is not surprising as high $C B K$ 
expression correlates with high HIFlA expression in human breast cancers, and HIF1A has previously been shown to be a negative prognostic factor associated with increased likelihood of metastasis in breast cancer patients [21, 76, 85]. Additional studies are needed to ascertain exactly how HIF- $1 \alpha$-dependent signaling of $\mathrm{Ckb}$ contributes to the stepwise process of metastasis, particularly if $\mathrm{Ckb}$ is not a direct HIF-1 transcriptional target.

Our findings suggest that $\mathrm{Ckb}$ is a major effector of in vivo HIF-mediated breast tumorigenesis and metastasis, indicating that targeting $\mathrm{Ckb}$ alone, or in addition to HIF$1 \alpha$, as part of combinatorial breast cancer treatment strategies may more effectively disrupt HIF-driven processes that contribute to aggressive tumor progression. 


\section{CHAPTER 7. USING HUMAN MODELS TO ELUCIDATE HIF-1 VERSUS HIF-2 CONTRIBUTION IN BREAST CANCER}

\section{Introduction}

A critical aspect of tumor physiology is the sensation of oxygen in the microenvironment. Over-expression of the oxygen-responsive Hypoxia-Inducible Factor (HIF) alpha subunits in breast cancer correlates with poor prognosis, increased risk of metastasis and decreased survival. Up to $17 \%$ of patients are diagnosed with triple negative breast cancer (TNBC; ER/PR/HER2-negative), however TNBC tumors account for at least $25 \%$ of breast cancer deaths [88]. In contrast to the luminal or HER2+ subtypes, no targeted therapies exist for TNBC. Additionally, the rapid onset of resistance to chemotherapy along with the spread of cancer to the liver, lung or brain is more common in women with TNBC. Comprehensive comparison of gene expression profiles among breast cancer subtypes has revealed that the hypoxic response transcriptional core signature is highly enriched in TNBCs as compared to luminal breast cancers $[92,93]$.

Recent studies from our laboratory have demonstrated an essential role of HIF-1 in promoting tumor growth and metastasis in a "luminal-like", ER negative, Neu/HER2 positive mouse model of breast cancer (Chapter 3). Yet, whether HIF1A and HIF $2 A$ perform different roles among the subtypes of human breast cancer remains unknown. Using human models of breast cancer, we are testing whether the HIF pathway promotes tumor growth and metastasis in luminal and triple negative breast cancers through differential regulation of HIF- $1 \alpha$ and HIF- $2 \alpha$ activity.

\section{Methods}

\section{Human Xenograft Maintenance}

The novel human tumor xenograft system developed in the laboratory of Dr. Alana Welm of the Huntsman Cancer Institute at the University of Utah authentically reflects the same tumor histopathology and the clinical patterns of metastasis observed in the patients from which the sample originated [132]. We have obtained cryopreserved tumor fragments from multiple sub-lines of tumors maintained in this tumor bank, including three triple negative (HCI-1, HCI-2 and HCI-10) and three luminal breast cancer (HCI-3, HCI-7 and HCI-11) lines, all of which have been successfully passaged in our laboratory into immunocompromised NOD/SCID gamma (NSG, NOD.Cg$\mathrm{Prkdc}^{\text {scid }} \mathrm{Il2} \mathrm{rg}^{\mathrm{tm} / \mathrm{Wjl}} / \mathrm{SzJ}$ ) female recipient mice. Frozen tissue chunks, obtained from Dr. Alana Welm, were briefly thawed and washed 3 times with DMEM, then briefly stored in DMEM/F12 media until time of transplant. An approximately $1 \mathrm{~mm} \times 2 \mathrm{~mm}$ sized piece of tissue was implanted into the inguinal fat pad of 3-6 week old female NSG mice. For $\mathrm{ER}^{+}$tumors, an estrogen pellet was implanted subcutaneously behind the neck at the 
same time as transplant surgery. Mice were monitored weekly for tumor growth. When tumors grew to $\sim 1,000 \mathrm{~mm} 3$, mice were euthanized and tumors harvested. Tumor fragments were either immediately used for re-transplant into another cohort of mice, cryopreserved for future use, frozen in liquid nitrogen for DNA, RNA and protein extraction or fixed in NBF and analyzed for histology. At necropsy, lungs and axillary lymph nodes were examined for gross metastases, and then fixed overnight in NBF.

\section{Cell Culture}

MDA-MD-231 cell culture was described previously in Chapter 4.

\section{Tumor Cell Transplants into NSG Recipients}

MDA-MB-231 cells were dissociated into single cells with $0.25 \%$ trypsin-EDTA and counted using a hemacytometer. Cells were resuspended in HBSS alone at a density of 250,000-1 million cells/10 ul. Cells were kept on ice until injection into the right inguinal mammary fat pads $(10 \mu \mathrm{l})$ of 3-6 week old female NSG recipients using a 26gauge PT2 needle mounted on a Hamilton syringe, followed by clearing of the endogenous epithelium. Recipients were palpated one or two times per week, and outgrowths were measured with digital calipers to calculate tumor volume as described previously [126].

For transplant of lentivirus-transfected HCI tumor cells, transduced cells cultured in suspension were pelleted at $600 \mathrm{rpm}$ to remove dead cells, washed once with HBSS and re-suspended in a 1:1 dilution of Matrigel:HBSS. The number of cells implanted into female NSG recipients was determined empirically for each experiment. All cells that survived antibiotic selection were transplanted into recipient mice (range of 4,000-45,000 cells per experiment). Due to the very low cell input, many months were required to regenerate tumors after viral transduction.

\section{Genetic Modification of HIFs in HCI Tumors}

Knockdown of either HIF $1 A$ or HIF $2 A$ (or co-deletion of both alpha subunits) was accomplished using pLKO.1 shRNA lentiviral vectors. Vectors used to create shHIF$1 \alpha$, shHIF-2 $\alpha$ and DKD MDA-MD-231 cells were based on shRNA sequences identified by Dr. Roland Wenger [26] and were also used to transduce cells derived from the HCI tumors. Tissues harvested from tumor bearing mice were cut into pieces using scalpels and minced with razor blades. All necrotic areas were removed prior to mincing of the tissue into a paste. The minced tissue was incubated with collagenase III (Worthington) and hyaluronidase (Sigma) and enzymatically digested in a $37^{\circ} \mathrm{C}$ shaker $(250 \mathrm{rpm})$ for 1 hour. Tissue was further digested to single cells as described in Chapter 3 . The final cell pellet was resuspended in serum free stem cell media (Chapter 3 ) plus the addition of heparin and allowed to recover overnight at $4^{\circ} \mathrm{C}$. The next day cells were plated at a 
density of 250,000 cells/well into 6-well ultra-low attachment plates. Lentivirus expressing firefly luciferase or luciferase and puromycin (pEILuc2 or

pEIPuro-Luc2, Figure 7-1) was added to the cells at an MOI of 10 in the presence of 8 $\mu \mathrm{g} / \mathrm{ml}$ polybrene. Virus was incubated with cells for 24 hours, and then cells were washed and replated in fresh stem cell media. After 48 hours, Luc2 positive cells were selected by the addition of $1 \mu \mathrm{g} / \mathrm{ml}$ puromycin (Figure 7-2). Once cells recovered from selection (5-7 days) they were pelleted, washed and resuspended in Matrigel for transplanting into NSG recipients.

Once Luc2+ tumors grew to sufficient size, flash frozen and cryopreserved fragments were prepared and the remaining tumor tissue was digested with the protocol above. Cells were transduced with either shHIF-1 $\alpha$, shHIF-2 $\alpha$ or shEmpty (EV) lentivirus. Cells were again selected, and live-cells re-transplanted back into the next generation of mice. Tumor growth was evaluated between shRNA and EV treated cells by caliper measurements and bioimaging.

\section{Xenogen Bioimaging}

D-Luciferin firefly potassium salt (Xenogen, XR-1001) was prepared in sterile DPBS without calcium and magnesium to a concentration of $30 \mathrm{mg} / \mathrm{mL}$. On imaging days, fresh stock solutions were diluted 1:2 with sterile DPBS to a final concentration of $15 \mathrm{mg} / \mathrm{mL}$. Mice received a dose of $10 \mu \mathrm{L}$ luciferin per gram of body weight by IP injection 10-15 minutes prior to imaging. Mice were anesthetized using isoflurane. The Xenogen Living Image software system was used to capture images. The camera settings were set to detect luminescence on auto exposure. Amount of luciferase signal was determined by total flux (photons/second) for each animal. Imaging was completed twice per week.

\section{Immunofluorescence}

Human xenografted tumors were harvested from mice and fixed in $10 \%$ neutral buffered formalin (NBF) for 6-8 hours at room temperature for immunostaining. Paraffinembedded sections $(5$ to $7 \mu \mathrm{m})$ were immunostained after antigen retrieval $(1 \times$ citrate buffer) using a rabbit polyclonal HIF-1 $\alpha$ antibody (Abcam 2185) at a dilution of 1:5000 followed by tryamide signal amplification (TSA) with Alexa Fluor ${ }^{\circledR}-488$ according to the manufacturer's protocol. (Invitrogen, T-20922). All sections were counterstained with DAPI and coverslipped using VECTASHEILD hard set mounting medium (Vector Laboratories).

\section{MDA-MB-231 Tumorsphere Assays}

MDA-MB-231 cells were prepared for tumorsphere assays as described in Chapter 3. Single cells were resuspended in serum-free $0.5 \%$ methylcellulose media 

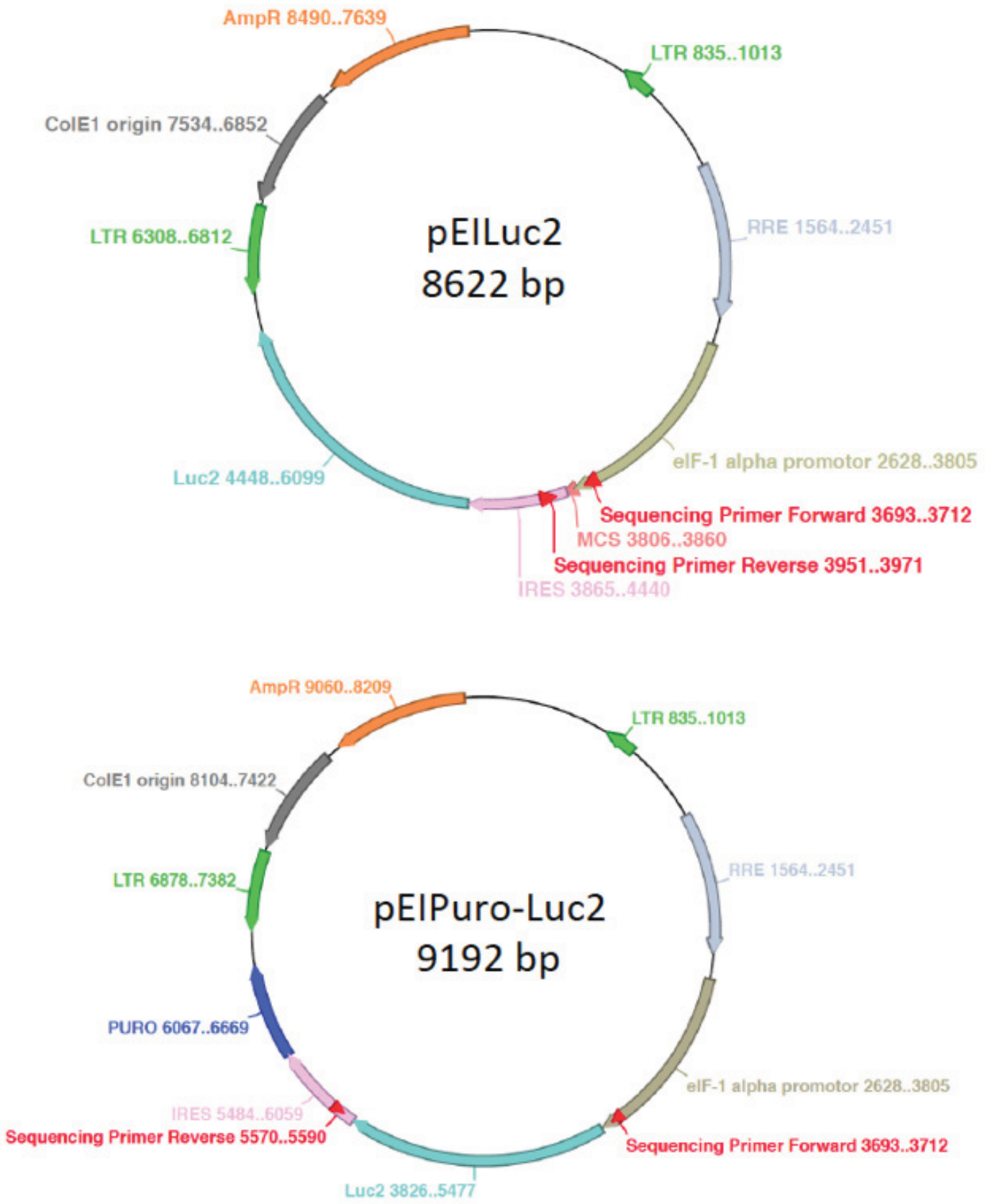

Figure 7-1. Maps of vectors used for luciferase labeling.

The parent vector, $\mathrm{pEILuc2}$ (top) was derived from the $\mathrm{pEIZ}$ vector previously obtained from Dr. Bryan Welm. The vector contains a multiple cloning site (MCS) and firefly luciferase coding sequence (Luc2, derived from the pGL4.13 vector from Promega) downstream of IRES, driven by the eIF-1 $\alpha$ promoter. Using this $p E I$ parent vector backbone, the puromycin resistance gene (Puro) was PCR cloned downstream of the IRES, and Luc2 was cloned into the MCS to create pEIPuro-Luc2 to allow for selection of cells expressing Luc2. All constructs have been reposited to Addgene by Dr. Bryan Welm. 


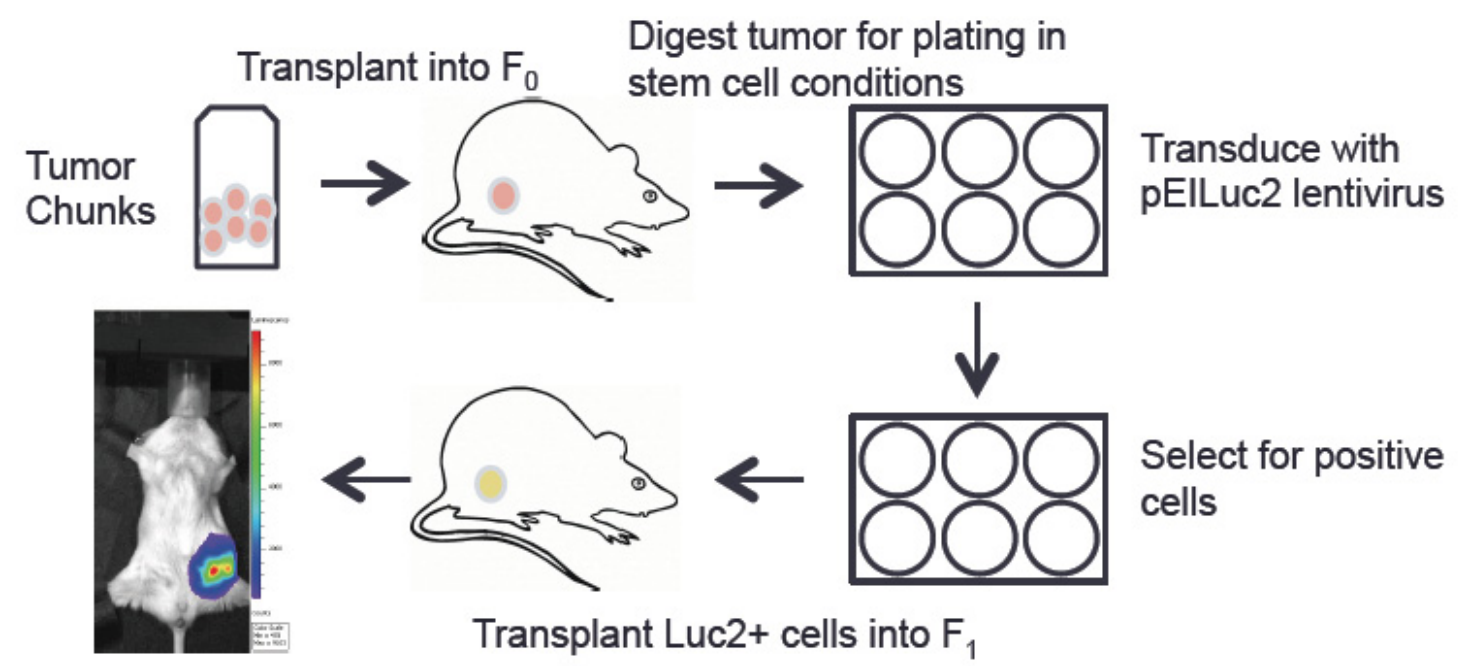

Figure 7-2. Schematic of lentivirus transduction of HCI tumors in stem cell conditions.

Fresh primary tissue chunks were transplanted into female NSG mice. At a tumor volume of $\sim 750 \mathrm{~mm}^{3}$, tumors were resected and digested to single cells for transduction with a luciferase expressing virus. Following transduction, live cells were transplanted into a new generation of female NSG mice, tumors were evaluated for growth by Xenogen live bioimaging. 
containing DMEM/F-12 plus $20 \mathrm{ng} / \mathrm{ml}$ mouse recombinant EGF, $20 \mathrm{ng} / \mathrm{ml}$ basic fibroblast growth factor, $1 \times$ B27 (all from Invitrogen) and $4 \mu \mathrm{g} / \mathrm{ml}$ heparin (SigmaAldrich, St Louis, MO, USA), as described previously [188]. Primary tumorspheres were derived by plating 500 single cells/well into 24 -well ultra-low-adhesion dishes. Dishes were cultivated at normoxia for 10 to 14 days prior to enumeration of spheres. Individual spheres $\geq 100 \mu \mathrm{m}$ from each replicate well ( $n \geq 12$ wells/genotype) were counted. The percentage of cells capable of forming spheres, termed the "sphere formation efficiency" (SFE), was calculated as follows: [(number of spheres formed/number of single cells plated) $\times 100]$.

\section{Results}

\section{HIF $\alpha$ Expression in Triple Negative Breast Cancer Models}

To determine level of expression of the HIF $\alpha$ proteins in various breast cancer subtypes, protein extracts from seven primary breast cancer PDX xenografts obtained from the HCI tumor bank were analyzed (Figure 7-3). The four TNBC tumors (HCI-1, 2, 9 and 10) expressed the highest levels of both HIF-1 $\alpha$ and HIF-2 $\alpha$, while the luminal tumors (HCI-5, 3 and 11) expressed lower levels of both proteins [1]. These data are consistent with previous findings that the HIF transcriptional core signature is enriched in TNBC [93]. To study the specific contribution of the HIF $\alpha$ subunits in the HCI samples, we procured 3 different TNBC and 3 different luminal cryopreserved tumor xenograft fragments from Dr. Alana Welm. Tumors were chosen based on levels of HIF $\alpha$ expression and considering the sites of distant metastases. Of the 3 TNBC tumors, 2 metastasize to lung and 1 metastasizes to axillary lymph nodes (AxLN). Of the 3 luminal tumors, 1 metastasizes to lung (HCI-11), 3 to the AxLNs and 1 to the bone (HCI-7). It has already been established that MDA-MD-231 cells express high levels of HIF-1 $\alpha$ as compared to other breast cancer cell lines [175].

\section{Transduction of HCI Tumor Cells with Lentivirus for Live Bioimaging and Evaluation of Metastatic Spread over Time}

In order to readily observe the growth rate of primary tumors and dissemination from the primary site to distant organs, we labeled HCI tumor cells with firefly luciferase (Luc2) by lentiviral transduction of cells cultured in stem cell conditions. Whole tumors were harvested from mice and digested to single cells in the absence of serum prior to transduction in stem cell culture conditions. The absence of serum and inability of cells to attach to tissue culture plastic has been reported to preserve gene expression and SNP profiles and histopathology of the primary patient samples [115, 273]. The first tumor I chose to label with Luc2 was the HCI-2 TNBC tumor, which expresses the highest levels of HIF $\alpha$ among all HCI TNBCs (Figure 7-2 and Figure 7-4). Live cells were injected into the 4R fat pad of 4 independent female NSG mice (passage 1, P1). Tumors were monitored weekly for growth and were harvested from mice when tumors reached a 


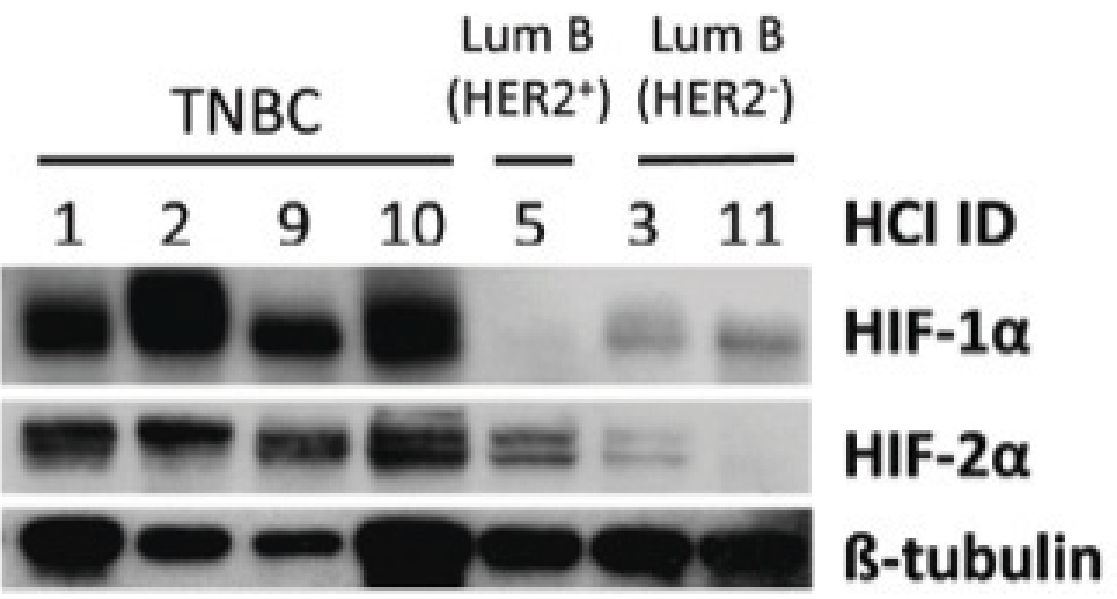

Figure 7-3. HIF $\alpha$ expression in HCI tumors of specific subtypes.

HS-WCE protein extracts were prepared from fresh frozen tumor tissues obtained from the laboratory of Dr. Alana Welm and probed for HIF- $1 \alpha$, HIF- $2 \alpha$ or $\beta$-tubulin (loading control).

Adapted from Anderson, T.M.R., et al., Breast Tumor Kinase (Brk/PTK6) is a mediator of hypoxia-associated breast cancer progression. Cancer Res, 2013. [1] 

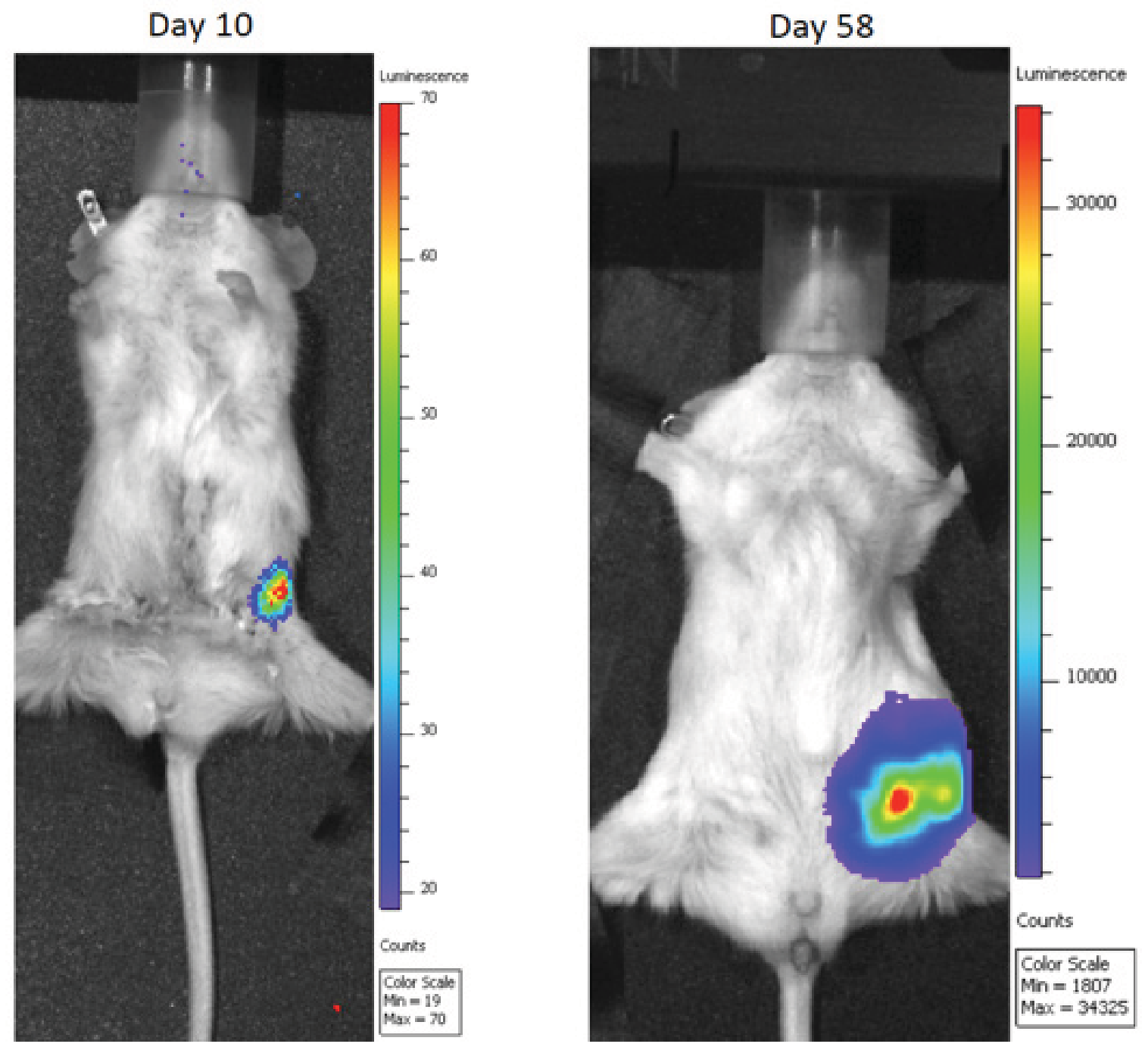

Figure 7-4. Xenogen bioimaging of HCI-2 Luc2+ tumor cells.

Tumors were harvested from mice bearing HCI-2 xenografts and digested to single cells prior to being plated in stem cell culture conditions for lentiviral transduction with pEILuc2. Twenty-four hours after transduction, cells were washed and replated in fresh stem cell medium and ultra-low attachment plates. After 14 days of growth in stem cell conditions, cells were pelleted and resuspended in a 1:1 mixture of HBSS:Matrigel, and injected into the inguinal fat pad of 3-6 week only NSG mice to generate parental (P1) tumors. Tumors were both palpated and imaged twice per week for a total of 9 weeks. Day 10 represents the first day Luc2 signal was detected by IVIS imaging, although no tumor masses were palpable. Day 58 represents the final Luc2 signal intensity just prior to tumor harvest; caliper measurements revealed a final tumor volume of $583.77 \mathrm{~mm}^{3}$ in this mouse. Images are representative of 4 individual mice. 
volume of 500-750 $\mathrm{mm}^{3}$. The individual HCI-2 Luc2+ tumor with the brightest Luc2 signal on the final day of bioimaging was used for digestion and transduction of cells with either pLKO.1-shHIF1A or empty pLKO.1 (EV) lentiviral particles. Following selection of shRNA targeted cells with puromycin, cells were transplanted back into the mammary fat pad of recipient NSG mice (P2). Due to the stress associated with virus transduction and antibiotic selection, very few cells were recovered for transplant from either the shHIF1A and EV cells. As a result only 2 recipient mice were used; each mouse received shHIF1A cells into the $4 \mathrm{R}$ fat pad and EV cells into the $4 \mathrm{~L}$ fat pad (Figure 7-5A). Moreover, the shHIF1A cells were recovered with $>5 x$ the yield compared to EV cells. Therefore, it was not expected tumors would grow at the same rate.

Evaluation of end-stage tumors revealed HIF-1 $\alpha$ protein levels were knocked down (as compared to EV controls in both mice) (Figure 7-5B). qRT-PCR analysis of HIF $1 A$ mRNA levels revealed a mean of $>80 \%$ knockdown for shHIF1A tumors (Figure 7-5C). Once tumors grew to approximately $750 \mathrm{~mm}^{3}$, they were harvested for retransplant into recipient mice to evaluate HIF-1 $\alpha$ knockdown on tumor growth in a larger cohort ( $\mathrm{n}=6$ tumors/genotype, $\mathrm{P} 3$ ).

Tumors were measured and imaged twice per week. Analysis of tumor volume over time indicated no obvious difference in the time palpable or measureable tumor onset between EV and shHIF-1 $\alpha$ tumors (Figure 7-6A). However, by day 30 post transplant, EV tumors began to grow at a faster rate then the shHIF-1 $\alpha$ tumors. Analysis of end-stage tumor volume revealed a significant reduction in tumor weight wet in the shHIF-1 $\alpha$ tumors as compared to the EV tumors (Figure 7-6B). Immunoblotting for HIF$1 \alpha$ protein levels among the individual end stage tumors suggested that knockdown of HIF-1 $\alpha$ with shRNA was not efficient as there was a large variation in the amount of HIF-1 $\alpha$ protein present among each knockdown tumor as compared to the EV control tumors. This finding was confirmed by immunofluorescence imaging which showed similar amounts of HIF-1 $\alpha$ protein in the various tumors as detected by western blotting (Figure 7-6D). Analysis of HIF-2 $\alpha$ protein levels by western blot did not reveal any significant change in expression between EV and shHIF-1 $\alpha$ tumors (data not shown).

Based on the relatively modest decrease in mean tumor growth and mean tumor wet weight in shHIF-1 $\alpha$ tumors as compared to EV controls, likely due to the high variability in HIF-1 $\alpha$ knockdown efficiency, we hypothesized that selecting the tumor with the best HIF $1 A$ knockdown would increase the difference in tumor growth between genotypes. Based on western blot results, EV \#1 and shHIF-1 $\alpha \# 3$ tumors were selected for re-passaging into recipient NSG mice (P4). Tumor growth was again evaluated over time. In contrast to the previous experiment, in this experiment the shHIF-1 $\alpha$ tumors grew faster than the EV tumors (Figure 7-7). Given our previous data using the PyMT model [127], and that this was our first experiment using a human model of breast cancer, we presumed there must have been a mistake in the experimental set-up. Prior to returning to using the HCI model, we next set out to evaluate HIF-1 $\alpha$ knockdown in a commonly used metastatic TNBC breast cancer cell line model, MDA-MB-231 cells. 

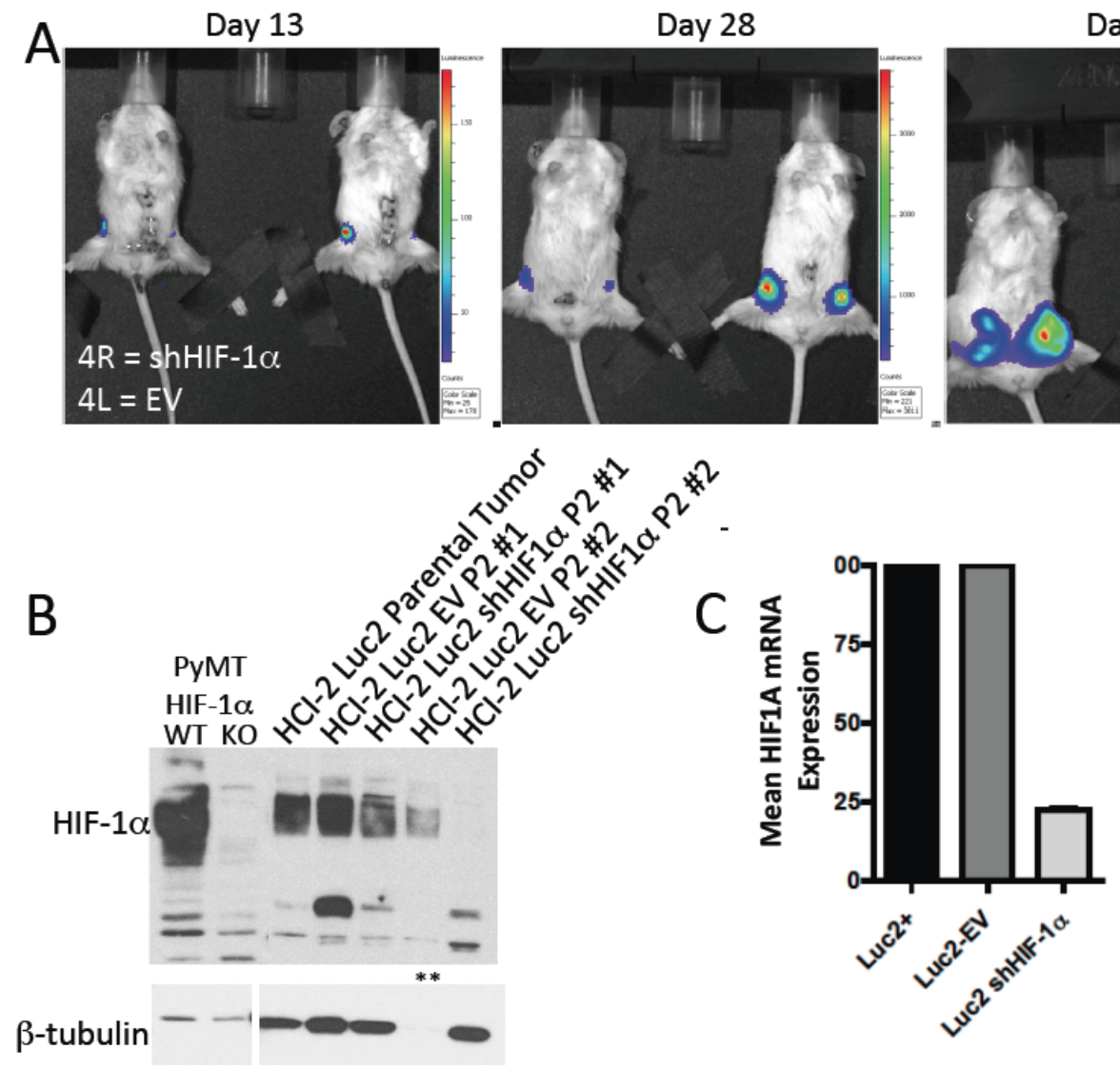

Figure 7-5. Xenogen bioimaging of HCI-2+shHIF-1 $\alpha$ or EV transduced tumors over time.

A. EV cells were injected into the $4 \mathrm{~L}$ inguinal fat pad and shHIF-1 $\alpha$ cells were injected into the 4R inguinal fat pad of 3-6 week NSG mice to generate second passage (P2) HIF1 wild-type (WT) and shHIF1A tumors, respectively. Tumors were both palpated manually and bioimaged twice per week for 9 weeks. Day 13 represents the first day to Luc2 signal was observed, although no masses were palpable. Day 28 shows signal midway through tumor growth and day 61 represents the final Luc2 signal observed prior to tumor harvest with an shHIF-1 $\alpha$ average tumor size of $1275.7 \mathrm{~mm}^{3}$ and an average EV tumor size of $764.67 \mathrm{~mm}^{3}$. B. Western blot of end-stage tumor HS-WCE probed for HIF$1 \alpha$;** represents under loaded protein for EV\#2 tumor. C. qRT-PCR analysis of mean HIFIA mRNA levels in tumors. 


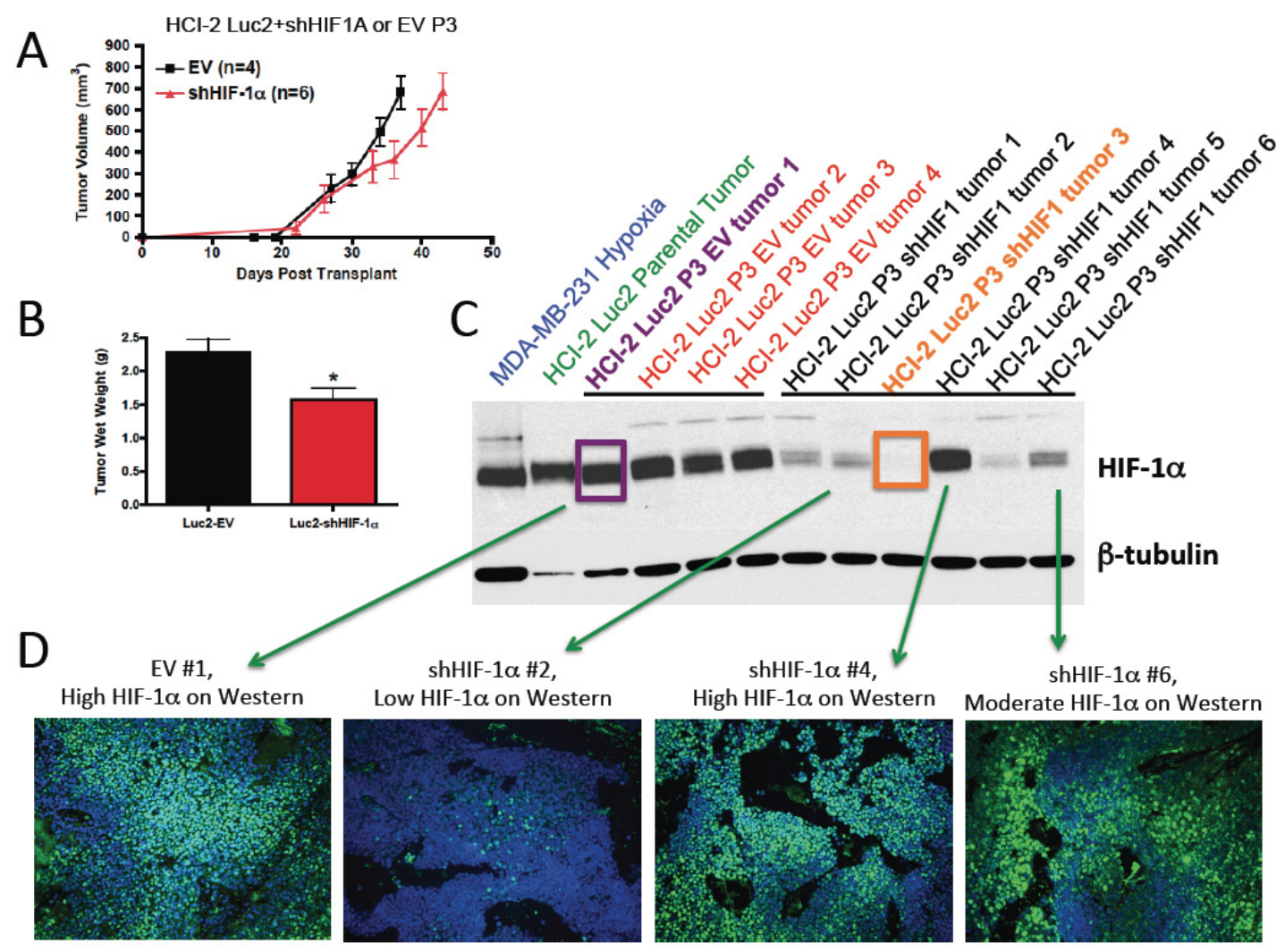

Figure 7-6. Comparison of tumor growth and HIF-1 $\alpha$ protein levels by western blotting and IHC in third passage HCI-2 tumors.

A. The growth rate of third passage (P3) HCI-2-Luc2 EV and shHIF-1 $\alpha$ tumors. B. All tumors were harvested and evaluated for end stage tumor wet weight $(p<0.05$, Students $t$ test). C. Western blot analysis of individual end stage P3 tumors for HIF-1 $\alpha$ protein levels D. Immunofluorescence staining of selected P3 tumors for HIF-1 $\alpha$. 


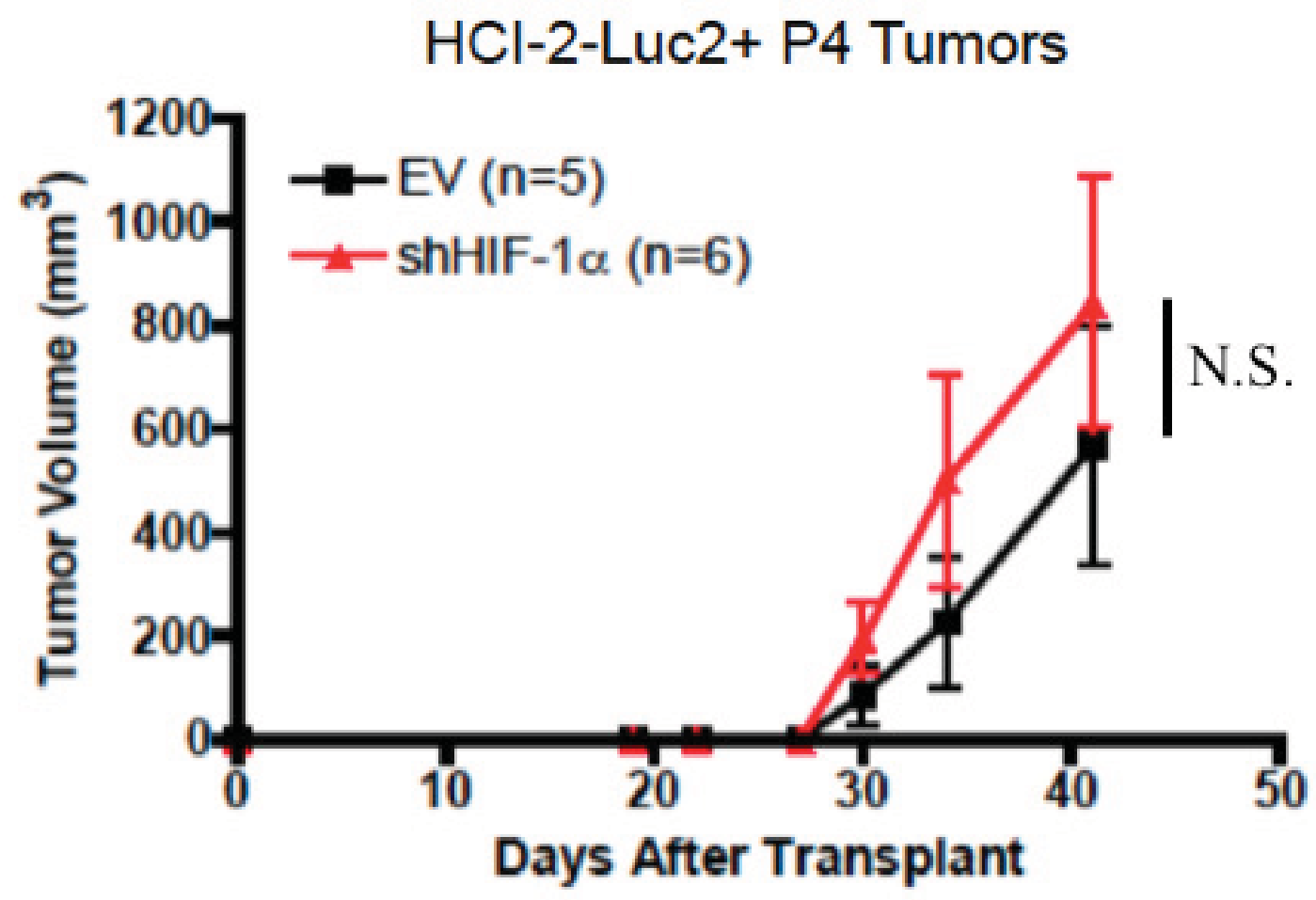

Figure 7-7. After selecting for the most efficient HIF1A knockdown HCI-2 tumor, shHIF1A repassaged $P 4$ tumors grow faster than $E V$ repassaged tumors.

Growth rate of HCI-2-Luc2 EV (\#1) and shHIF-1 $\alpha$ (\#3) tumors repassaged from P3, now P4) selected for highest and lowest HIF-1 $\alpha$ expression, respectively. Data show a trend that knockdown of HIF1A accelerates tumor growth, but the difference between means is not significant at any time point by two-way ANOVA with Bonferroni correction. 


\section{HIF1A Knockdown in MDA-MB-231 Cells Increases Primary Tumor Growth}

We obtained in collaboration with Dr. Roland Wenger MDA-MB-231 cells, in which either $H I F 1 A, H I F 2 A$ or both genes had been knocked down to generate shEmpty (EV/WT), shHIF-1 $\alpha$, shHIF-2 $\alpha$, and shHIF-1 $\alpha$ /shHIF-2 $\alpha$ (DKD) cells. We verified that all lines had $>80 \%$ knockdown of either HIF $\alpha$ gene in single or double knockdown cells (Figure 7-8A-C). To investigate the invasion potential of cells knocked down for HIF $\alpha$, we performed an invasion assay through a Matrigel layer. While shHIF-1 $\alpha$ cells had a modest increase in the ability to invade, shHIF-2 $\alpha$ cells had a significant increase invasion with no change in DKD as compared to EV at normoxia. The ability of the shHIF-2 $\alpha$ cells to invade further increased when exposed to hypoxia, while the invasion potential of the DKD cells decreased. These results were obtained from a single experiment and need to be repeated. A tumorsphere assay was then utilized to examine the sphere forming ability of each cell line. In 3 independent experiments we observed a significant increase in the sphere forming potential of shHIF-1 $\alpha$ cells as compared to EV controls, while there was only a slight but not significant increase in shHIF-2 $\alpha$ cells. DKD cells had almost no sphere forming potential; suggesting the ability to form spheres is highly dependent on the expression of at least one HIF $\alpha$ subunit. The ability of cells to form spheres in stem cell cultures is generally associated with tumor initiation potential, therefore these data support our in vivo findings in the HCI-2 tumors.

Next all 4 genotypes of MDA-MB-231 cells of NSG mice were transplanted into the mammary fat pad at a density of 250,000 cells. shHIF-1 $\alpha$ tumors grew twice as fast as all other genotypes and were be harvested 10 days earlier due to reaching a study endpoint size of $>1000 \mathrm{~mm}^{3}$ tumor volume (Figure 7-9A-B). In contrast, HIF-2 $\alpha$ knockdown significantly decreased tumor growth as compared to EV, and DKD further decreased growth (Figure 7-9C). Analysis of end stage tumors revealed an increase in HIF-2 $\alpha$ protein and mRNA levels in the shHIF-1 $\alpha$ tumors (Figure 7-9D-E), while HIF$1 \alpha$ levels remained low, leading us to propose that HIF-2 $\alpha$ is driving the growth of these tumors. However, HIF- $2 \alpha$ knockdown was not maintained well in vivo, but shHIF-1 $\alpha$ and DKD are maintained. These results are in contrast to what we expected to discover given our previous data with the PyMT model. However, these results are consistent with what we observed when HIF $1 A$ was knocked down in the HCI-2 tumors, suggesting differing roles for HIF-1 $\alpha$ and HIF-2 $\alpha$ in promoting tumor growth and progression in different subtypes of breast cancer. Construction of stabilized HIF-1 $\alpha$ and HIF-2 $\alpha$ lentiviruses to generate $H I F 1 A$ and $H I F 2 A$ overexpression (Pro-Ala mutations) in MDAMB-231 cell lines is in process to test this hypothesis.

\section{Characterization of Tumor Growth and Metastasis Using the TNBC HCI-10 Tumor Line}

Our studies performed using the HCI-2 TNBC tumors, suggested that HIF-1 $\alpha$ knockdown increases tumor growth. Knockdown of HIF-1 $\alpha$ in MDA-MB-231 TNBC 


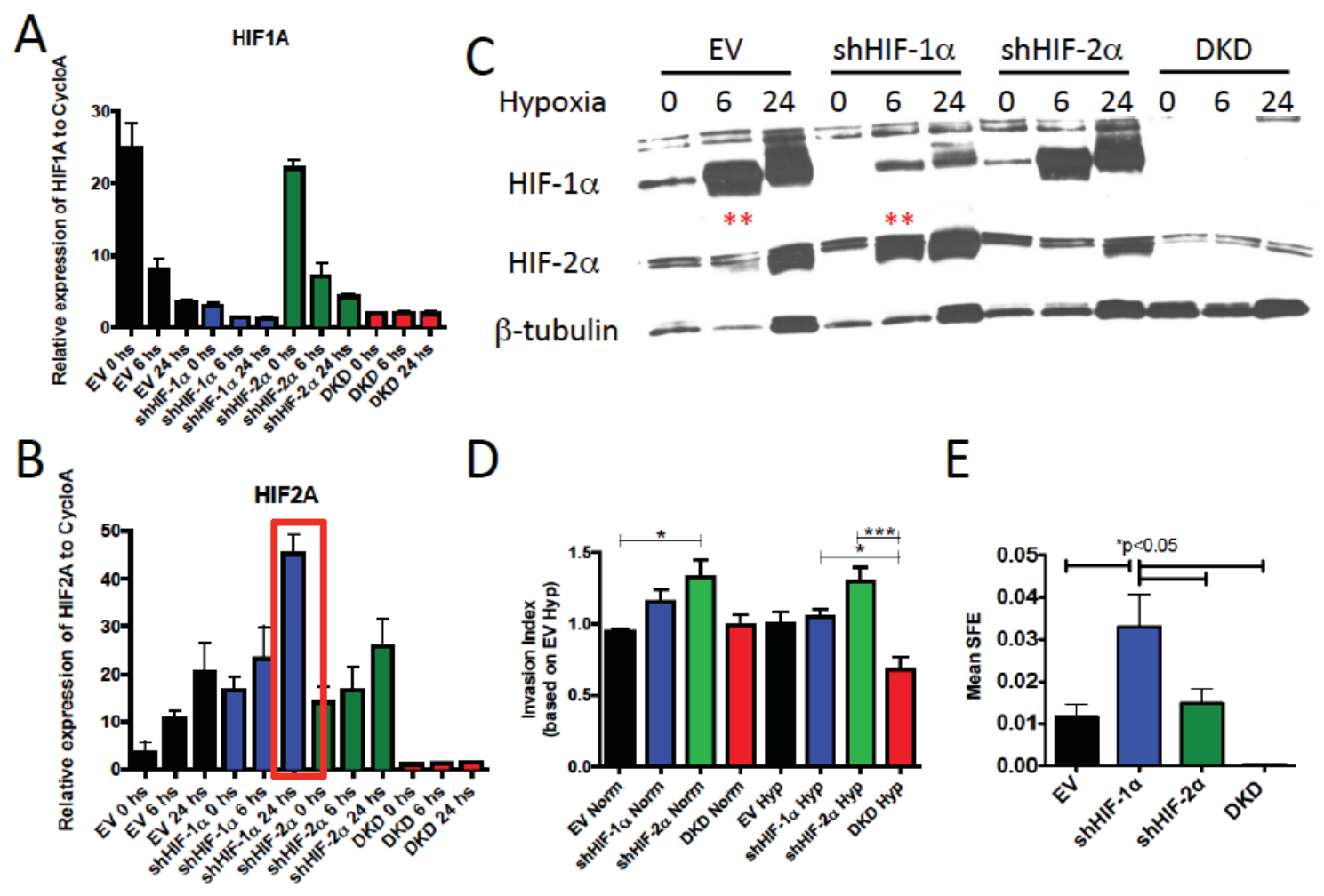

Figure 7-8. Characterization of HIF $\alpha$ knockdown phenotypes in MDA-MB-231 cells.

A. HIF $1 A$ and B. HIF $2 A$ mRNA levels in MDA-MB-231 knockdown cells at 24 hours normoxia or 6 to 24 hours hypoxia $\left(0.5 \% \mathrm{O}_{2}\right)$. Values are relative mRNA levels as compared to expression of Cyclophillin A (loading control). B. HS-WCE extracts from cultured cells were blotted for HIF-1 $\alpha$, HIF- $2 \alpha$ and $\beta$-tubulin (loading control) to determine HIF $\alpha$ protein levels in the knockdown cell lines. D. The mean fold-change in invasion was normalized to the invasion index observed for EV cells cultured at hypoxia $(\mathrm{FC}=1.0)$. Data represent the mean fold change in invasion observed in a single pilot experiment. All columns were compared to each other using one-way ANOVA analysis with a Bonferroni post-test, ${ }^{*} p<0.05, * * * p<0.001$. E. The mean sphere formation efficiency $(\mathrm{SFE}) \pm$ S.E.M. was determined when cells were cultured in tumorsphere culture conditions. Tumorspheres $\geq 100 \mu \mathrm{m}$ were scored positive, and the mean SFE (the percentage of cells capable of forming spheres per total number of single cells plated) per experiment ( $\mathrm{n} \geq 12$ wells/genotype; $\mathrm{p}<0.05$, unpaired Student's t-test) was determined. Data represents mean of three independent tumorsphere experiments. 


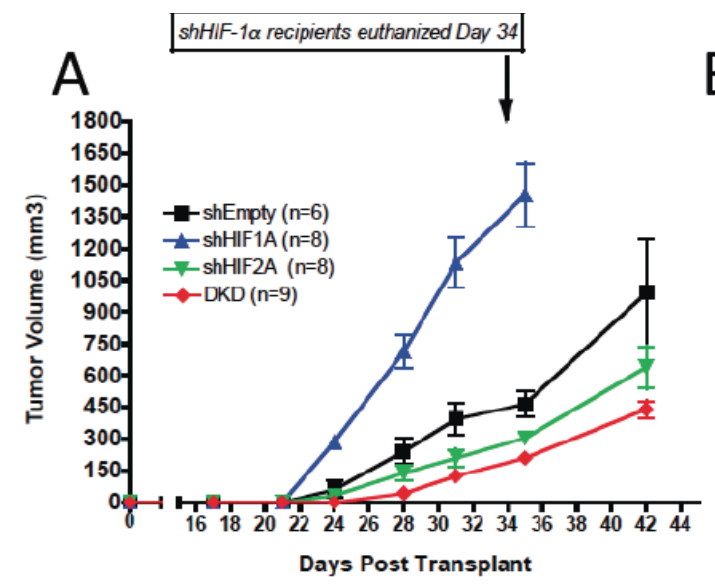

B

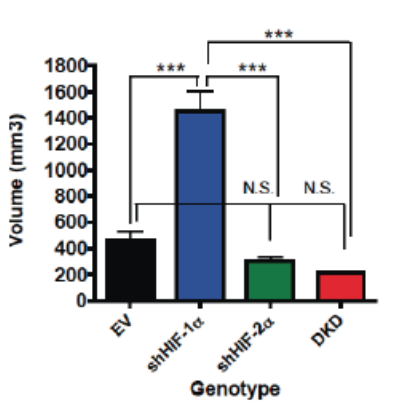

E

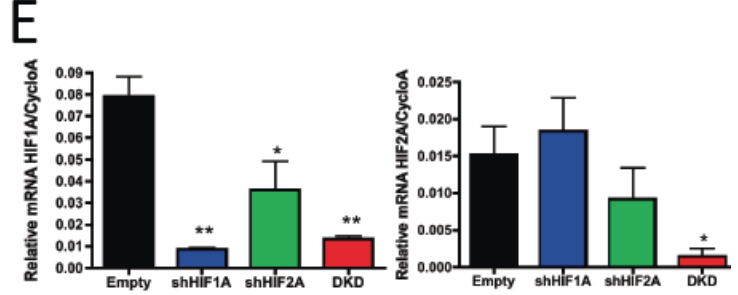

Figure 7-9. Phenotypes following knockdown of HIF-1 $\alpha$ and HIF-2 $\alpha$ in MDAMB-231 cells.

A. The growth rate of MDA-MB-231 EV, shHIF- $1 \alpha$, shHIF- $2 \alpha$ and DKD tumors at 250,000 cells input. B. Day 34 tumor volume just prior to sacrifice of shHIF-1 $\alpha$ tumors $(* * * p<0.001$, ANOVA with Bonferroni correction). C. Evaluation of tumor volume at endpoint without shHIF- $1 \alpha$ tumors $\left(* * p<0.01,{ }^{*} p<0.05\right.$ ANOVA with Bonferroni correction). D-E. HIF-1 $\alpha$ and HIF-2 $\alpha$ protein and mRNA levels in end-stage knockdown tumors $(* * p<0.01, * p<0.05$ ANOVA with Bonferroni correction). 
cells also supported these findings. However, neither of these models demonstrates highly efficient spontaneous lung metastases from the primary mammary tumor in the mammary fat pad, as we observe in the PyMT model. Therefore we chose to refocus efforts to compare the roles of HIF-1 versus HIF-2 in a more metastatic TNBC model. The HCI-10 tumors are capable of metastasizing to the axillary lymph nodes from the mammary fat pad. In order to perform bioimaging analysis we also revised the Luc2 labeling process by creating the $\mathrm{pEIP-Luc2}$ vector so that Luc2 + cells could be selected by puromycin resistance (Figure 7-1). This refinement should result in a more evenly labeled Luc2+ tumor cell populations.

HCI-10 tumor cells were transduced with pEIP-Luc2 lentiviral particles in stem cell conditions and Luc2 + cells were selected for with $1 \mu \mathrm{g} / \mathrm{mL}$ of puromycin. Cells remained in culture for 14 days prior to being pelleted, washed and resuspended in Matrigel for injection into the fat pad of female NSG mice. Approximately 180,000 cells were divided evenly and injected into both number 4 fat pads of 2 independent mice $(\mathrm{n}=45,000$ cells/injection). Twice weekly bioimaging and palpation revealed Luc2 signal as early as 13 days after transplant prior to any palpable mass detected in the fat pad (Figure 7-10A-C), indicating bioimaging is more sensitive in monitoring tumor progression. By day 57 there was a robust Luc2 signal in each transplanted fat pads, although the mammary tumor was only just palpable and not yet measureable with calipers (Figure 7-10B-C). Moreover, by day 57 post transplant, the first signal was detected in the axillary lymph nodes suggesting that metastasis occurs very early in tumorigenesis in the HCI-10 model. By the last day of imaging, a robust signal was observed in both axillary lymph nodes of animal \#2241. When mice were harvested for tumor removal, we observed there were metastases in both axillary lymph nodes at a size of approximately 2-3 $\mathrm{mm}$ in diameter. Overall, these data demonstrate that we could visualize the formation of AxLN metastases before there was a palpable tumor mass, and we could correlate a signal intensity with increasing metastatic burden. Upon necropsy, it was confirmed that the signal observed by bioimaging was indeed from an AxLN metastases and not from lung metastases. In order to confirm that this was not an isolated event, we retransplanted tissue chunks from the first passage (P1) HCI-10 Luc2+ tumors into recipient NSG mice to generate passage 2 (P2) HCI-10 Luc2+ tumors. We again observed the same pattern of tumor growth and AxLN metastasis over time (Figure 7-11).

To determine if HCI-10 Luc2+ tumors also increase in tumor growth rate upon HIF1A deletion and what the effects of HIF- $2 \alpha$ deletion would be, we are currently in the process of transducing these HCI-10-Luc2 tumors with shHIF- $1 \alpha$ and ShHIF- $2 \alpha$ lentivirus. HCI-10 Luc2+ EV transduced cells have been generated, and tumor fragments are cryopreserved for future use. 


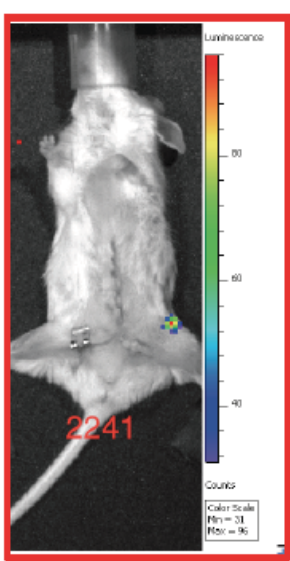

Day 13

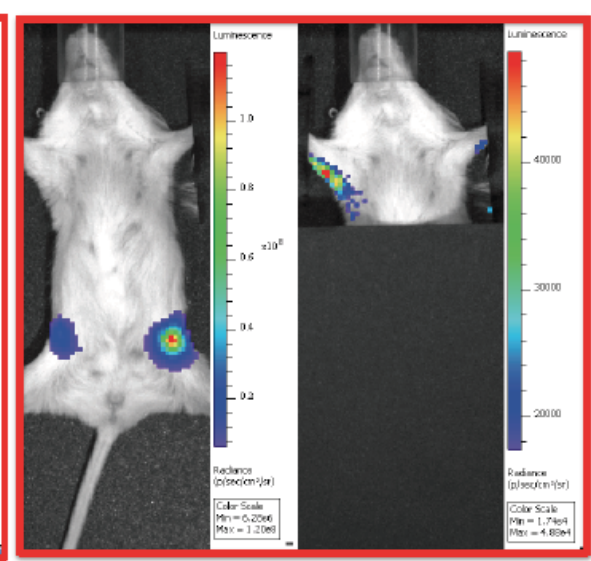

Day 57

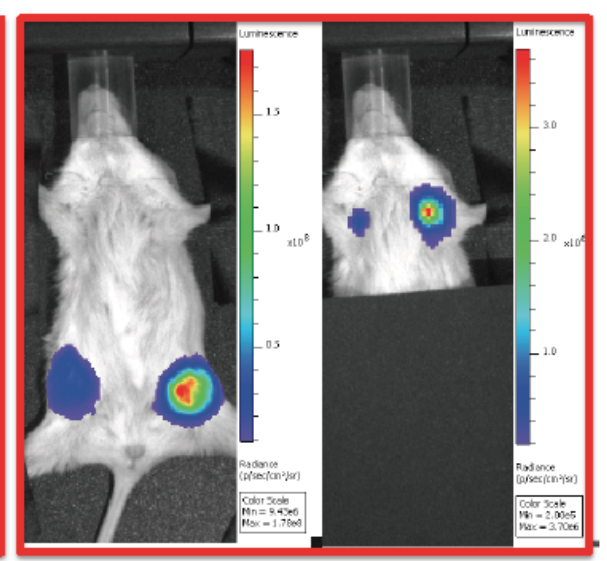

Day 111
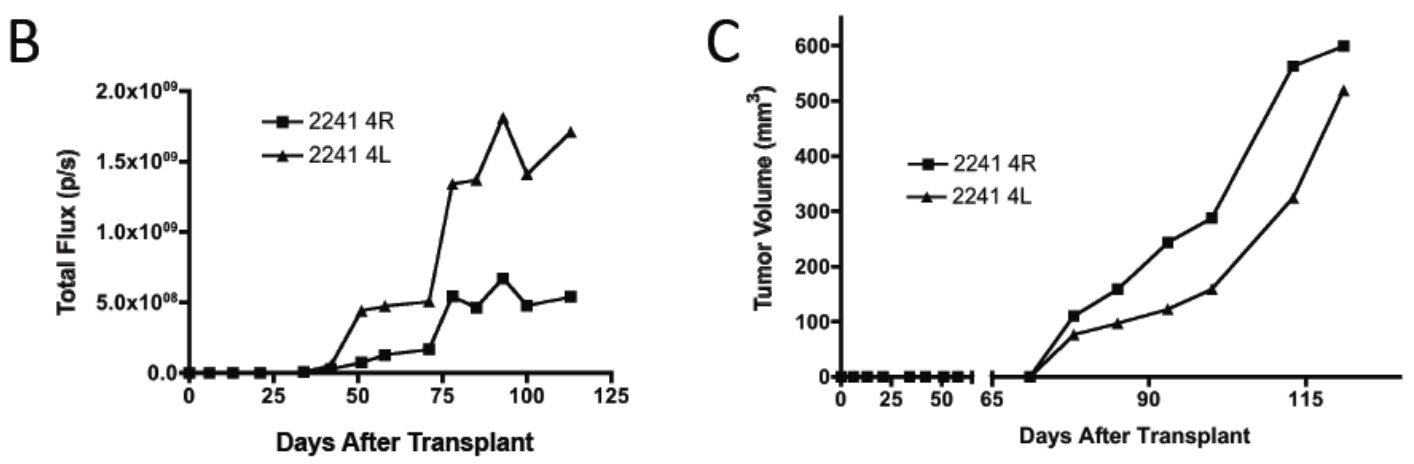

Figure 7-10. HCI-10 Luc2+ P1 tumor growth and metastasis over time.

A. Tumors were harvested from mice, digested to single cells, transduced with pEIPLuc2 and selected for with $1 \mu \mathrm{g} / \mathrm{mL}$ puromycin prior to being injected into the inguinal fat of 3-6 week old NSG mice. Tumors were both palpated and bioimaged twice per week. Day 13 represents the first day Luc2 signal was observed, with no palpable tumor mass. Day 57 represents a middle time point for which tumors were palpable but were not measureable with calipers and axillary lymph node metastases were first apparent. Day111 represents a late stage HCI-10 Luc2+ signal just prior to harvest with robust signal detected in both the primary tumor and the axillary lymph node metastases. B. Plot of Luc2 signal (total flux, photons/second, p/s) over time post transplant. C. Plot of tumor volume over time post transplant. 


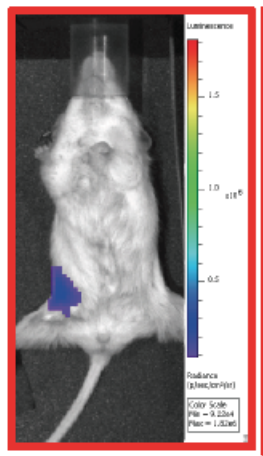

Day 21

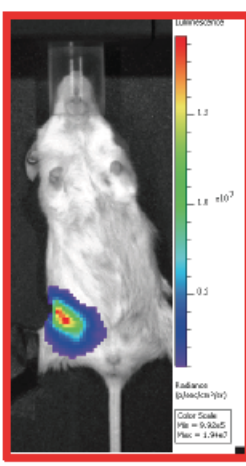

Day 34

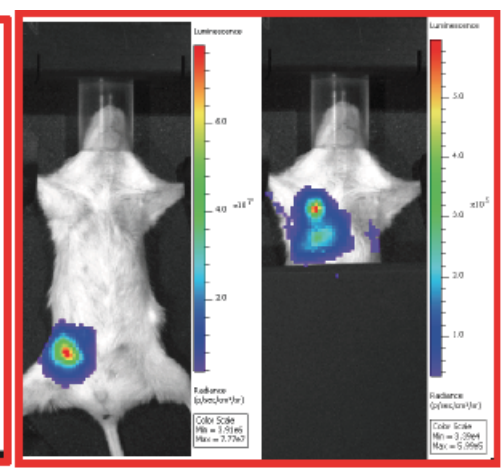

Day 62

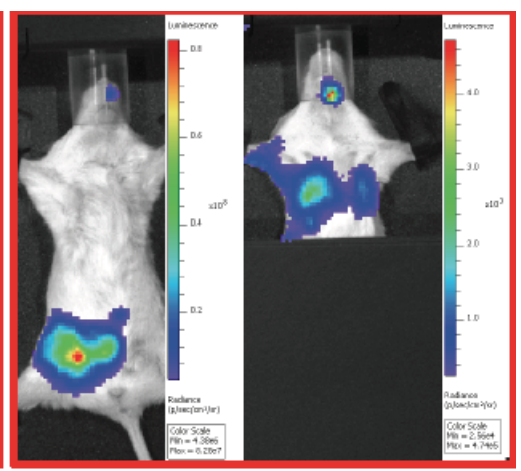

Day 89
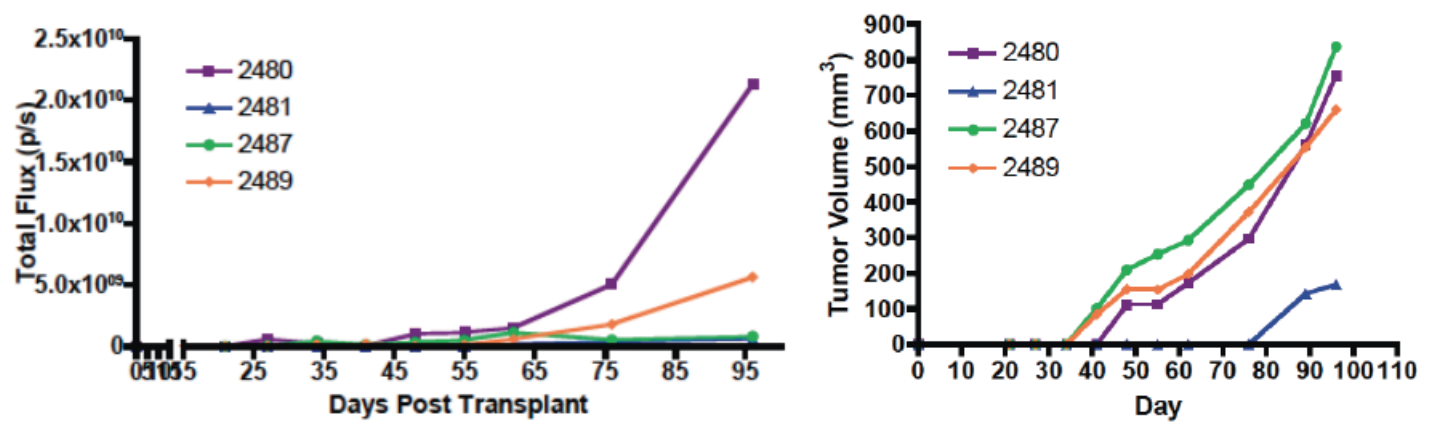

Figure 7-11. Characterization of P2 HCI-10 Luc2+ tumor and AxLN metastasis by bioimaging.

HCI-10 Luc2+ tumors were harvested from hosts and tumor fragments were immediately repassaged into the inguinal fat pad of 3-6 week old NSG mice. Tumors were both palpated and imaged twice per week. Day 13 represents the first day Luc2 signal was observed, with no palpable tumor mass. The upper panel shows tumor growth and metastasis development over time (images are representative of 4 animals). The lower panels display Luc2 signal (total flux, $\mathrm{p} / \mathrm{s}$ ) and tumor volume over time for the duration of the experiment. 


\section{Discussion}

We have previously generated evidence to support a positive role of HIF-1 $\alpha$ in breast cancer initiation, progression and metastasis (Chapters 3, 4, and 6 and Schwab et al [127]). However, the exact mechanism of HIF-2 $\alpha$ in these processes remains to be established. We have demonstrated that in the luminal-like, MMTV-PyMT model of breast cancer, deletion of HIF-1 $\alpha$ results in a decrease in tumor initiation potential, tumor growth and metastasis. However, when we knocked down HIF-1 $\alpha$ in the TNBC HCI-2 tumor, we observed the opposite effect. In these TNBC tumors, sufficient loss of HIF-1 $\alpha$ $(>80 \%)$ is required for increased tumor growth as compared to HIF-1 $\alpha$ WT (EV) controls. In the triple negative human breast cancer cell line, MDA-MB-231, we verified these findings, while also observing that HIF-2 $\alpha$ knockdown had only modest though statistically significant effects on primary tumor growth. Additionally, tumor sphere assays suggested that loss of HIF- $1 \alpha$ conferred a more stem-like nature in the cells by significantly increasing tumorsphere formation efficiency (SFE), while loss of HIF-2 $\alpha$ did not affect SFE. These conflicting results obtained from the PyMT model and these two different TNBC models of breast cancer suggest that the HIF-1 and HIF-2 may play different roles in promoting tumor initiation, progression and metastasis, and that these roles may also be dependent on the molecular subtype of the breast tumor (TNBC versus luminal).

In order to validate these preliminary findings, we are investigating the effects of HIF $\alpha$ knockdown in a third triple negative tumor model, HCI-10. Knockdown of the HIFs in this tumor will also allow us to evaluate the consequences HIF $\alpha$ loss has on early metastases as these tumors develop lymph node metastases very early during tumorigenesis. The HCI-10 tumor line has been robustly labeled with luciferase to facilitate observation of HIF $\alpha$-dependent differences in metastatic phenotypes. Additionally, I am in the process of labeling three HCI luminal tumors (HCI-3, HCI-7, and HCI-11) with luciferase, and to then be subjected to gene knockdown for the HIF $\alpha$ subunits. Once the luminal knockdown tumors are established, we will be able to ascertain whether or not these luminal tumors behave like the PyMT model or if they portray a different phenotype and behave more similar to HCI TNBC and MDA-MB-231 cells. With these reagents we will be able to more clearly delineate the exact roles of both HIF-1 and HIF-2 in tumor growth and metastasis in different subtypes of human breast cancer. 


\section{CHAPTER 8. DISCUSSION}

A considerable body of research has been accumulated relevant to the biology of HIF- $1 \alpha$ in breast cancer. As early as the 1920's metabolic maintenance in the presence of varying oxygen levels was being described in cancers [274]. Metabolic homeostasis is important for expansion under the low oxygen, or hypoxic conditions for tumors [275]. It was not until more than half a decade later, however, that HIF-1 $\alpha$ was identified as being the transcriptional regulator of hypoxia-induced expression of erythropoietin $(E P O)$ [276, 277]. It was quickly realized that HIF-1 was a master regulator of the hypoxic response $[190,278]$. Following this discovery, rapid advances were made in delineating the role of HIF- $1 \alpha$ in tumorigenesis and metastasis. The first demonstrations that HIF1 mediated tumor-promoting effects occurred only a few years after it was purified and cloned [41, 279]. Subsequent studies continued to compile evidence that increased levels of HIF$1 \alpha$ are directly and positively associated with increased cell invasion, metastasis and death [3], as well as resistance to chemotherapy, immunotherapy and radiotherapy [160]. Moreover, high HIF-1 $\alpha$ expression has been found to independently correlate with poor overall survival, increased risk of relapse and increased risk of metastasis in breast cancer patients [85]. While significant progress has been made in understanding how hypoxia and the HIF-dependent hypoxic response influence tumorigenesis, substantial work is left to fully define this complex pathway. In this body of work, I have delineated the role that the HIFs play in breast cancer initiation, progression and metastasis through regulation of breast cancer TIC activity and gene expression.

\section{The HIFs Regulate TICs}

We first characterized the role of Hifla in regulating breast TICs by using an ex vivo genetic deletion approach in the MMTV-PyMT mouse model of breast cancer [127]. In this model we generated constitutive HIF- $1 \alpha \mathrm{KO}$ and WT mammary tumor epithelial cells that were useful both for culture in vitro and for transplantation back into immunocompetent hosts to generate HIF-1 $\alpha$ WT and KO mammary carcinomas. Using this approach, we were able to demonstrate that HIF- $1 \alpha$ is required for primary mammary tumor growth and lung metastasis [127]. Additionally, I was the first to demonstrate that HIF-1 $\alpha$ regulated TIC activity in breast cancer, in part through up regulation of the Notch pathway through assays detailed in Chapter 3. Further investigation into the role of HIF- $1 \alpha$ in TIC activity, led us to the discovery that HIF- $1 \alpha$ directly regulates many genes currently used to enrich for cancer stem cells, including CD133 and CD24, although other laboratories published these data before our work was published $[117,224]$. We recently observed that another commonly used marker for stem cells, CD49f/ITGA6, was highly dependent upon HIF expression. Furthermore enrichment for $\mathrm{CD} 49 \mathrm{f}^{\text {high/+ }}$ cells by FACS not only enhanced primary tumor growth, but also increased lung metastases, as described in Chapter 4. These data provide compelling evidence that HIF regulates cancer stem cells, as has been shown in lymphomas [60] and gliomas [115]. 
TICs are believed to modulate phenotypes associated with metastatic breast cancer cells, including enhanced migration/invasion and enhanced resistance to chemotherapy [163]. Therefore, targeting the CSC/TIC population for eradication could have a significant impact on patient prognosis and therapeutic response. Additionally, TICs are more resistant to therapy than bulk tumor cells or the surrounding stroma [162, 280], although the exact mechanisms for this resistance are not well defined. Moreover, independent of its role in CSCs/TICs, HIF-1 $\alpha$ was previously shown in multiple tumor types to contribute to resistance to radiation and chemotherapy [281-284]. A recent study suggests one underlying mechanism may involve HIF-1 $\alpha$ 's direct regulation of the multidrug resistance (MDR) gene [14]. These data, combined with our findings, suggest a prominent role for the HIF $\alpha$ subunits in regulating TICs in a variety of solid tumors, making this pathway an attractive therapeutic target. Furthermore, the enrichment of HIF $\alpha$ protein expression in TNBCs versus luminal cancers $[1,92,93]$, may directly regulate the increased aggressiveness and chemoresistance phenotypes observed in TNBCs, in part by directly regulating the TICs. Treating patients diagnosed with TNBC is challenging, since tumors are typically high grade, patients do not response to endocrine therapies or Herceptin, and onset of chemotherapy resistance to conventional chemotherapies is rapid, leading to a higher risk of recurrence and decreased 5-yr survival [88]. To date no FDA-approved targeted therapies exist for. Therefore, it is possible that HIF inhibitors, or inhibitors to genes downstream of HIF in the hypoxic response, may be particularly beneficial to TNBC patients.

As another example, targeting CD49f, which is enriched in TNBC (Figure 4-6) [229] and is a direct HIF target (Figure 4-3), within these tumors may provide therapeutic benefit. Recently, multiple novel therapeutic systems have been designed with the aim of killing CSCs and altering the microenvironment (niches) supporting these cells [285]. For example, a monoclonal antibody against CD133 (gemtuzumab ozogamicin) has been developed and used to successfully treat patients with AML [286]. In addition, targeting Notch, which promotes CSC activity [287, 288], has been effective in lowering the CSC population, in addition to lowering the incidence of brain metastases from a breast cancer cell line [289]. Utilizing strategies such as these, we may be able to target CD49f gene expression or integrin signaling to treat patients diagnosed with TNBC whose tumors express high levels of CD49f. Increased expression of CD49f during treatment may also be useful as a biomarker that predicts onset of resistance to therapy or metastatic relapse.

\section{The HIFs Promote Tumor Metastasis}

In addition to playing a definite role in regulating TIC phenotypes, the HIFs are also involved in breast cancer metastasis. The hypoxic response is clinically relevant to metastatic breast cancer since hypoxia stimulates extravasation, anoikis and colonization [25]. As described in Chapter 3, I showed that HIF-1 $\alpha$ is a key driver of the formation of lung metastases originating from primary PyMT mammary tumors [127]. When lungs were harvested from mice at the same time as primary tumor removal, very few lung metastases were present. However, when lungs were harvested 8-weeks after primary 
tumor removal, mice bearing HIF-1 $\alpha$ KO tumors showed a $90 \%$ decrease in the formation of lung metastases. In contrast, when cells were injected directly into the tail vein, there was no difference in lung metastatic events between mice receiving HIF-1 $\alpha$ WT or KO cells (unpublished data). These data provide some insight into which of the various steps of the metastatic cascade at which HIF-1 $\alpha$ is necessary to promote metastasis. Only after we remove the primary tumor do we see a significant effect on lung metastases, suggesting that the HIF pathway is stimulated by surgery, perhaps because surgery activates the wound response. These observations are consistent with what has been reported in the clinic, that surgery can induce cancer metastasis [290]. However, the distinct lack of a difference in phenotype between WT and KO PyMT cells when studying metastasis using the tail vein model, suggests that HIF-1 $\alpha$ plays a more pronounced role in the early stages of metastasis in this model.

There are multiple steps early in the metastatic cascade where HIF could be required. For example, at what point do cells start entering the circulation? Are cells being shed from the primary tumor once it reaches a certain volume, and because HIF-1 $\alpha$ KO tumors grow more slowly, they do not shed as many cells? A recent paper by the Semenza laboratory demonstrated that HIF-1-dependent activation of collagen prolyl hydroxylases promotes local invasion and intravasasion of breast cancer cells [291]. Are there HIF-dependent cytokines or other factors released when the primary tumor is removed that amplifies this process? Additionally how well do cells survive once they enter the blood stream?

Since disseminated cells are no longer in the breast microenvironment, they may require some sort of survival signal to survive long enough to make it to distant organs. How does HIF mediate cell survival in this setting? Data from our collaboration with the Reginato lab suggests that HIF promotes survival in the blood stream as single cells by preventing anoikis [90]. Moreover, cells need to receive a signal from distant organs in order to seed them. This process, known as homing, may require different HIF signals at different organ sites. For example, Schito et al demonstrated that HIF-1 promotes lymphatic metastasis by directly activating platelet-derived growth factor B (PDGF-B), and PDGF-B is required for cells to attach to lung endothelium [292].

While we have not yet studied these exact mechanisms, the HIF-1 $\alpha$ WT and KO PyMT tumor model may prove to be a useful tool in answering these questions using syngeneic, immunocompetent mice. Collecting blood from mice during the course of tumor development, from transplant initiation to tumor endpoint, could be used to analyze the occurrence and frequency of circulating tumor cells between HIF-1 $\alpha$ WT and $\mathrm{KO}$ tumor bearing mice. Additionally, cytokine and growth factor arrays performed on these blood samples or from conditioned media from cultured HIF knockdown cells would determine if there are differentially excreted proteins necessary for cell intravasion and survival between WT and KO, similar to [293]. Investigation into these processes could ultimately lead to the identification of additional HIF pathways amenable for therapeutic target for patients with metastatic breast cancer. 


\section{A Novel HIF-Dependent Gene Involved in Breast Cancer Progression and Metastasis}

The microarray profiling screen we performed on HIF-1 $\alpha$ WT and KO cells and the microarray screen on late stage WT and KO tumors took us one step closer in answering some of these questions and identified several differentially regulated HIF-1 $\alpha$ genes and pathways involved in tumor development. Analysis of the array data revealed that HIF-1 $\alpha$ regulates multiple genes involved in stem cell maintenance, as discussed above and in Chapter 5. Many other genes we initially identified have since been implicated as being HIF-1 $\alpha$ dependent genes such as Angptl4 [72], Jarid1b and the Jumanji proteins [246], several pro-collagens [291] and $A x l$ [245]. However, we also discovered several genes that still had not been previously explored as hypoxiadependent or in the context of breast cancer. One of these genes was $C k b$.

My studies into the HIF-1 $\alpha$ dependent expression of $C k b$ (as discussed in Chapter 6) have shown that while $\mathrm{Ckb}$ expression is not necessary for $2 \mathrm{D}$ cell growth, it is necessary for cell invasion through Matrigel. Loss of $\mathrm{Ckb}$ in vivo led to a reduced time to primary tumor development and significantly reduced the occurrence of lung metastases, both after primary tumor removal and in a tail vein model. Furthermore, mice injected with PyMT WT cells treated with the $\mathrm{Ckb}$ inhibitor, cyclocreatine (cCr), displayed a marked decrease in lung metastases versus control mice treated with saline only.

Further investigation into $\mathrm{Ckb}$ revealed that not all breast cancer cell lines express $\mathrm{Ckb}$ at the protein level. Western blotting for CKB in MDA-MB-231 cells produced no detectable CKB signal, while MCF7 cells expressed moderate levels (data not shown). Investigation into $\mathrm{CKB}$ expression in the $\mathrm{HCI}$ tumor series revealed that $\mathrm{CKB}$ is highly expressed in all samples, although at various levels. Additionally, prior microarray profile screening performed on MCF7 HIF- $1 \alpha$ and HIF- $2 \alpha$ knockout cells has demonstrated a substantial decrease in $C K B$ mRNA levels in HIF-1 $\alpha$ knockout cells, but not in HIF-2 $\alpha$ knockout cells [294], In the same dataset, $C K B$ was also found to cluster with other known HIF-1 $\alpha$ target genes like BNIP $3 L$ and $L O X$ (personal observations).

These data are exciting as $\mathrm{Ckb}$ is highly expressed in a variety of tumors and is HIF-1-dependent. HIF-driven, hypoxic areas are a hallmark of most solid tumors and their metastases. Despite advances in early detection and therapies for some types of breast cancers, patients with advanced, recurrent metastatic breast cancer (MBC) have $<$ $20 \% 5$-year survival rates. In phase I, clinical trials, $\mathrm{cCr}$ therapy did not demonstrate any severe toxicity and, therefore, may not dose-limiting. Additionally, cCr has previously been shown to prevent pathological bone loss [295]. Therefore, in addition to its chemotherapeutic power, $\mathrm{cCr}$ could also be used to ameliorate bone loss due to osteolytic lesions. However, no trials testing efficacy of cCr therapy have been performed in breast cancer patients. Given that $\mathrm{cCr}$ is relatively inexpensive as compared to other mainstream chemotherapies, these data provide evidence for the hypothesis that targeting HIF and HIF-dependent genes like CKB could be beneficial in the development of new anticancer therapeutics, especially for those with metastatic breast cancer. 


\section{The Differing Roles of the HIFs in Breast Cancer}

HIF- $1 \alpha$ is important for many aspects of tumor initiation, progression and metastasis. As discussed briefly in Chapter 4 and in further detail in Chapter 7, we have shown that HIF-2 $\alpha$ plays an important role in breast cancer in addition to HIF-1 $\alpha$. We found that CD49f, which is involved in both tumor initiation and metastasis, is regulated by both HIF-1 and HIF-2. Upon the loss of HIF-1 $\alpha$ in MDA-MB-231 cells, HIF-2 $\alpha$ is able to compensate for loss of HIF-1 $\alpha$ and will induce transcription of CD49f. This could be explained by our observation that in the TNBC 231 cell line, HIF-2 $\alpha$ protein levels increase in HIF-1 $\alpha$ knockdown cells. However, we did not observe this effect in our luminal-like, Neu/HER2+ PyMT cell line, which express relatively low levels of HIF-2 $\alpha$ versus HIF- $1 \alpha$ in contrast to MDA-MB-231 cells (data not shown). These results suggest that the roles of HIF-1 $\alpha$ and HIF-2 $\alpha$ may be breast cancer subtype dependent. This hypothesis is further supported by our findings that in both the TNBC HCI-2 tumor xenograft model (Figure 7-7) and the TNBC MDA-MB-231 cell line (Figure 7-9), knockdown of HIF-1 $\alpha$ leads to an increase in tumor growth and metastases as compared to HIF-1 $\alpha$ WT tumors. These data are again in contrast to our findings in the luminal-like PyMT model where knockout of HIF-1 $\alpha$ led to decreased tumor growth and metastasis, without a compensatory increase in HIF-2 $\alpha$.

Further investigation into the differing roles of the HIFs in different subtypes of breast cancer could lead to a better understanding of how these tumors would respond to certain therapies, and help to develop more targeted therapies for patients with a particular subtype of breast cancer. I predict that use of the HCI model will be most useful in delineating these roles since this system most accurately models human breast cancer and metastasis. Although we have utilized the TNBC HCI-2 xenograft tumors to knockdown HIF-1 $\alpha$, we have not yet studied the effects of HIF-2 $\alpha$ knockdown in these tumors. It is unknown if loss of HIF-2 $\alpha$ would give the same phenotype as we observed in the TNBC MDA-MB-231 cell line, or if these primary patient samples would behave differently. Furthermore, as HCI-2 and the other TNBC HCI tumors are derived from different patients, it is possible that while they are the same subtype, they themselves may contain intrinsic differences they may result in different HIF-1 $\alpha$ and HIF-2 $\alpha$ knockdown phenotypes.

Additionally, the luminal HCI tumors still require evaluation of the phenotypes resulting from loss of the HIFs. However, we have encountered technical difficulties in establishing stem culture and lentiviral transduction conditions in these tumors. One of the main caveats is the slow growth these tumors exhibit in vivo. It can take many months before tumors are large enough to be harvested and digested. Our first attempts at digesting the luminal tumors into single cells resulted in a very low cell yield and after only 2 days in stem cell conditions, most cells had died. Furthermore, the nature of some of these tumors makes it very difficult to extract single cells, as most of the single cells from particular lines, such as HCI-11, are trapped in a mucus-like substance after enzymatic digestion. We have take steps to circumvent these problems, such as having

multiple tumor bearing mice in our animal colony at all times and adding estradiol to the 
digestion and stem cell medias, since these tumors are highly dependent upon estrogen. In addition, forcing cells out of the mucus substance through a filter has aided in increasing the final cell yield. Yet, we are still in the process of establishing technical parameters to generate HIF- $1 \alpha$ or HIF- $2 \alpha$ knockdown luminal tumors.

One additional caveat of all of our experimental approaches is the repeated use of gene knockdown for the HIF $\alpha$ subunits, when what is actually observed in patients is gene overexpression. Therefore, approaches that overexpress the HIF $\alpha$ subunits in human breast cancer cell lines or the HCI xenografts could provide additional insight into the roles of HIF-1 $\alpha$ and HIF- $2 \alpha$ in the different subtypes of breast cancer. Our lab has obtained pBabe vectors for both HIF-1 $\alpha$ and HIF-2 $\alpha$ overexpression (OE) in which the proline residues hydroxylated by PHD enzymes are mutated to alanine residues [296]. These mutations disable the PHDs from being able to target the HIF $\alpha$ subunits for degradation, therefore rendering the HIFs stabilized and constitutively active. We have successfully transduced the HIF-2 $\alpha$ OE lentiviral vector into MDA-MB-231 cells, and are working on transducing them with the HIF-1 $\alpha$ OE vector. The next step would then be to test tumorigenesis and metastatic phenotypes in all of the MDA-MB-231 HIF genotype cells. Once all of these tumor lines are established, we would have invaluable tools to fully define the differing roles the HIFs play in primary patient breast tumors. Moreover, since the HCI tumors replicate the same patterns of metastasis in the mouse as the patient, we could also discern distinct roles for the HIFs in the metastatic process of these tumors, and if there is a correlation between HIF $\alpha$ expression and subtype in terms of metastatic potential.

\section{A Role for the HIFs in Stromal Cells in Supporting Breast Cancer Progression and Metastasis}

In addition to the roles HIF plays in tumor cells, HIF-1 is also required for mobilization of mesenchymal stem cells (MSCs) into peripheral blood [269]. MSCs produce pro-inflammatory cytokines in response to hypoxia [297], which may potentiate breast tumor cell TIC activity. One mechanism is that MSCs produce IL-6, which activates STAT3 in the breast tumor cell, stimulating breast TIC activity and the production of additional IL-6 in a feed forward loop [297]. A direct or necessary effect of the HIF transcription factors in coordinating these pathways in breast cancer in either cell type (tumor or MSC) has not been investigated. It is possible that HIF-1 and/or HIF-2 expression is required in both the tumor cell and in host MSCs for maximal induction of tumor-initiation, angiogenesis and metastasis.

To determine if hMSCs co-transplanted with tumor cells promotes tumor initiation through HIF-dependent mechanisms, via an IL-6/STA3 loop, or via stimulation of tumor angiogenesis, or both, utilization of the HCI model in which the HIFs are knocked down would provide an excellent tool. MSCs WT or KD for the HIFs coinjected into the mammary fat pads in combination with HCI tumors WT or KD for the HIFs, would help to determine if HIF $\alpha$ expression is critical in the tumor cell, hMSCs host cells, or both cell types. These experiments would not only give insight into hMSC- 
dependent effects on the effect of HIF $\alpha$ deletion on cytokine production, but also if there is an enrichment of TICs in the presence of hMSC and if there is an increase in metastasis potential. Given our findings on the importance of HIF in breast cancer cells, we would expect to find that HIF-1 and/or HIF-2 is also required in hMSCs to promote tumor growth through regulation of both TIC activity and angiogenesis, suggesting more than a tumor cell-intrinsic role in modeling the tumor microenvironment. In this case, strategies to inhibit HIF activity in combination with Stat3 inhibitors may represent a novel, targeted therapeutic approach to treat breast cancer by eradicating the population of breast cancer TICs that drive relapse and therapeutic resistance and/or by blocking positive feedback loops between tumor cells and stromal cells (MSCs).

\section{Summary of Major Findings}

1. Overexpression of HIF-1 $\alpha$ upon VHL deletion is not sufficient to induce mammary tumorigenesis during normal mammary gland development.

2. Ex-vivo deletion of Hifla in mammary tumor epithelial cells in the MMTVPyMT model of breast cancer results in delayed primary tumor growth and decreased lung metastases originating from the mammary fat pad.

3. HIF- $1 \alpha$ regulates breast cancer TIC activity, likely in part through regulation of the Notch pathway.

4. HIF-1 $\alpha$ directly regulates transcription of the breast cancer stem cell marker ITGA6/CD49f, which promotes tumor initiation and metastasis.

5. ITGA6 is an independent prognostic factor for breast cancer patient survival.

6. There are several HIF-1 $\alpha$ regulated genes in breast cancer that regulate processes such as stem cell maintenance, angiogenesis, cell invasion and metastasis that may be useful as therapeutic targets in the future.

7. $C k b$, a HIF-1 $\alpha$ dependent gene, promotes primary tumor growth, cell invasion and metastasis to the lung. Treatment of cells expressing Ckb with cyclocreatine inhibits the formation of lung metastases. $\mathrm{CCr}$ may be useful therapy for patients with MBC.

8. CKB mRNA is an independent prognostic factor for breast cancer patient survival.

9. Expanding analysis of HIF-dependent genes in breast cancer to human models, including the HCI "living tissue" bank and conventional breast cancer cell lines, revealed differing roles for the HIF $\alpha$ subunits in breast cancer development and progression. Whether HIF-1 $\alpha$ or HIF- $2 \alpha$ promotes or attenuates tumorigenesis and metastasis in human breast cancer may depend on the molecular subtype 
classification. 


\section{CHAPTER 9. REMAINING QUESTIONS AND FUTURE EXPERIMENTS}

In Chapters 1-8 I described the role that the HIFs play in breast cancer initiation, progression and metastasis. While I have produced valuable insights to further delineate the role of HIF-regulated genes in MBC, several questions remain to be answered in the models I have created and characterized. In answering the questions posited below, we will develop a more comprehensive understanding of the exact function of HIF-regulated genes in breast cancer progression and metastasis.

\section{Is CD49f Alone a Driver of Tumor Progression and Metastasis, or Does it Require the Presence of CD24?}

Dr. Bryan Welm has observed in the PyMT model that only CD24 ${ }^{\text {hi }}$ cells retain TIC activity (personal communication). Therefore it is possible that the absence of CD24 in our experiment $\left(\mathrm{CD} 24^{\text {low }}\right.$ paired with $\left.\mathrm{CD} 49 \mathrm{f}^{\text {dow }}\right)$ may be confounding the results that CD49f enriches for TICs. In order to address this issue, CD24 $4^{\text {hi }}$ PyMT cells would be used as the parent population to enrich only for the CD49f high or low cell subpopulations and the sorted cells used to repeat the limiting dilution transplantation assay (as presented in Chapter 4). Given my data using CD49f sorted MDA-MB-231 cells in a tail vein assay, in which I only enriched for CD49f, I would expect to observe a similar phenotype in the PyMT cells enriched only for CD49f, including more metastatic burden in a tail vein assay and enriched TIC potential in CD49 $\mathrm{f}^{\text {high }}$ cells by the limiting dilution transplantation assay.

\section{Can Re-Expression of Ckb in PyMT HIF-1 $\alpha$ KO or shc61 KD Cells Rescue the Tumor and Metastasis Phenotypes?}

PyMT HIF-1 $\alpha$ KO cells, which express low levels of Ckb (Chapter 6), exhibit decreased mammary tumor growth and lung metastasis (Chapter 2). Therefore, ectopic expression of $\mathrm{Ckb}$ in $\mathrm{KO}$ cells or re-expression of $\mathrm{Ckb}$ in shc61 knockdown cells may increase tumor growth and promote metastasis to levels observed in HIF-1 $\alpha$ WT cells, which express high levels of $\mathrm{Ckb}$. To address this question, a lentiviral expression vector expressing the complete coding sequence of murine $\mathrm{Ckb}$ with a N-terminal Flag tag (FLAG-Ckb) will be introduced in HIF-1 $\alpha$ KO cells or shc61 KD cells. These cells will then be evaluated for the expression of the Flag protein to ensure induced expression of $\mathrm{Ckb}$ over residual endogenous $\mathrm{Ckb}$ expression. Cells will then be used in a primary tumor growth experiment with HIF- $1 \alpha$ WT and constitutive HIF- $\alpha$ KO cells. It is expected that the HIF- $1 \alpha \mathrm{KO}+$ FLAG-Ckb tumors will grow at similar rates as the HIF-1 $\alpha$ WT tumors, providing additional evidence that HIF-1-dependent Ckb is a key driver of tumorigenesis. 


\section{Is the Ckb shRNA Targeting Sequence (c61) Specific to Ckb, or Are There Off- Target Effects?}

Since off-target effects can be observed when using shRNA mediated knockdown technology, additional controls may be required during peer review. Since most of the data in Chapter 6 was generated using shRNA knockdown, an additional control to support my findings would be to utilize an shRNA-resistant $C k b \mathrm{cDNA}$. In this experiment, translationally silent substitutions have been made in four nucleotides within the c61 shRNA targeting sequence in $C k b$, therefore inhibiting binding of the c61 shRNA. This c61-shRNA-resistant $C k b$ cDNA will be ectopically expressed in PyMT HIF- $1 \alpha$ KO cells, followed by transduction with the c61 shRNA. If the shRNA targeting sequence is specific, then I would expect there to be no knockdown in the cells that ectopically express the c61-resistant sequence. In addition, if these cells do not efficiently generate primary tumors or metastases by tail vein assay, then this would strongly suggest that the c61 shRNA targets other genes besides $\mathrm{Ckb}$ that are responsible for the c61 shRNA phenotypes observed using WT cells that express c61 shRNA.

\section{Can Treating Primary Tumors with Cyclocreatine Inhibit Primary Tumor Growth as well as Lung Metastases?}

I demonstrated in Chapter 6 that as an alternative to $C k b$ gene knockdown, treating cells expressing $\mathrm{Ckb}$ with the chemical inhibitor cyclocreatine also results in a significant decrease in lung metastases. Therefore, I hypothesize that treating mice with primary tumors with cyclocreatine would also result in a decrease in mammary tumor growth and potentially inhibit lung metastasis from the primary tumor site. Complementary, cyclocreatine could be tested using the HCI xenograft model and conventional metastatic breast cancer cell lines for potential primary tumor and metastasis anticancer activity. To address this question, I have xenografted recipient NSG mice with tumor fragments from the HCI-10 Luc2 model. Once tumors are palpable, mice will be treated daily with either saline $(n=10)$ or $1 \mathrm{~g} / \mathrm{kg}$ cCr by i.p. injection $(n=15)$, and bioimaged twice a week to monitor tumor growth and AxLN metastases.

\section{Is CKB a Direct HIF Target Gene?}

Since no conclusive results could be obtained from initial ChIP experiments using the PyMT MTECs and the Santa Cruz ChIP grade antibody, experiments will be repeated using the PyMT MTECs immunoprecipitated with the Abcam antibody, which may have higher affinity for mouse HIF-1 $\alpha$. Additionally, MCF7 are currently being analyzed for HIF-1 $\alpha$ binding at two independent HRE sites to further support our findings. 


\section{Are There Additional Understudied Genes Revealed by Array Profiling That Could Provide Additional Targets for Successful Breast Cancer Therapy?}

The identification of $C k b$ using the microarray data as a novel HIF-dependent gene that promotes MBC phenotypes, has led to an exciting potentially clinically useful strategy. There are several other genes derived from the analysis of the arrays that have not yet been functionally characterized for a role in breast tumorigenesis, such as Emb, Elf5 and Mial, that could also lead to additional insights into the biology of HIFdependent tumorigenesis and metastasis. Emb (Embigin) is a member of the immunoglobulin superfamily that functions as a cell adhesion molecule [298]. As many cell adhesion molecules are disregulated in cancer, further investigation into this gene could provide addition insight into cancer cell motility. Elf5 is part of the epitheliumspecific subclass of the Ets transcription factor family, and is involved in regulating many epithelial-specific genes [299]. In particular Elf5 is enriched in normal mammary gland luminal progenitor cells [300], and also regulates the EMT [301]. Mia1 has previously been implicated in regulating invasion and metastasis of malignant melanoma cells [302] and also functions as an attachment protein in the extracellular matrix [303]. Further investigation into these genes in breast cancer may lead to the discovery of a novel target that promotes metastasis.

\section{Are There Additional Cells Lines to Use as Models That Would Replicate My Results Derived from MDA-MB-231 Cells?}

The conflicting data generated from our PyMT model and the MDA-MD-231 model with respect to the function of HIF-1 versus HIF-2, suggests that additional cell line models must be evaluated for HIF $\alpha$-dependent activities. Our lab is currently in the process of profiling an extensive panel of different breast cancer cell lines available from ATCC that are representative of the major molecular intrinsic subtypes by western blotting in order to determine if any lines express similar levels of the HIF $\alpha$ proteins as MDA-MB-231 cells. If we identify another line with similar expression levels of HIF-1 $\alpha$ and HIF- $2 \alpha$ as the MDA-MB-231 cells, we will determine if the same tumor phenotypes are observed in vitro and in vivo following HIF $1 A$ and HIF $2 A$ knockdown. This approach will also be useful to explore the role of HIF-1 versus HIF-2 HER2-enriched tumors, as we currently maintain only a single PDX model (HCI-7).

\section{Which Additional Studies Can Be Performed to Better Define the Potentially Opposing Roles of the HIF $\alpha$ Proteins in TNBC?}

I have generated data showing that HIF-1 and HIF-2 may have different roles in TNBCs. Using the four HIF $\alpha$ deletion genotypes created in the MDA-MB-231 cell line, two important experiments could be performed. The first would be to perform ChIP-Seq on the cells to identify HIF-1 versus HIF-2 specific targets, and to determine if HIF-1 targets increase with deletion of HIF2A and vice versa. The second would be to perform RNA-Seq to profile RNAs, including mRNAs, IncRNAs, microRNAs, that are 
differentially regulated between genotypes at both normoxia and hypoxia. Data obtained from these experiments could help delineate specific pathways and genes that are up and down regulated with HIF-1 $\alpha$ and HIF-2 $\alpha$ expression or deletion. 


\section{LIST OF REFERENCES}

1. Anderson, T.M.R., et al., Breast Tumor Kinase (Brk/PTK6) is a mediator of hypoxia-associated breast cancer progression. Cancer Res, 2013.

2. Simon, M.C., et al., Hypoxia-induced signaling in the cardiovascular system. Annu Rev Physiol, 2008. 70: p. 51-71.

3. Brizel, D.M., et al., Oxygenation of head and neck cancer: changes during radiotherapy and impact on treatment outcome. Radiother Oncol, 1999. 53(2): p. 113-7.

4. Bertout, J.A., S.A. Patel, and M.C. Simon, The impact of O2 availability on human cancer. Nat Rev Cancer, 2008. 8(12): p. 967-75.

5. Chaplin, D.J., R.E. Durand, and P.L. Olive, Acute hypoxia in tumors: implications for modifiers of radiation effects. Int J Radiat Oncol Biol Phys, 1986. 12(8): $\mathrm{p}$. 1279-82.

6. Rankin, E.B. and A.J. Giaccia, The role of hypoxia-inducible factors in tumorigenesis. Cell Death Differ, 2008. 15(4): p. 678-85.

7. Semenza, G.L., Hypoxia-inducible factor 1: master regulator of O2 homeostasis. Curr Opin Genet Dev, 1998. 8(5): p. 588-94.

8. Gray, L.H., et al., The concentration of oxygen dissolved in tissues at the time of irradiation as a factor in radiotherapy. Br J Radiol, 1953. 26(312): p. 638-48.

9. Nordsmark, M., M. Overgaard, and J. Overgaard, Pretreatment oxygenation predicts radiation response in advanced squamous cell carcinoma of the head and neck. Radiother Oncol, 1996. 41(1): p. 31-9.

10. Tannock, I.F., Conventional cancer therapy: promise broken or promise delayed? Lancet, 1998. 351 Suppl 2: p. SII9-16.

11. Durand, R.E., The influence of microenvironmental factors during cancer therapy. In Vivo, 1994. 8(5): p. 691-702.

12. Yu, J.L., et al., Effect of p53 status on tumor response to antiangiogenic therapy. Science, 2002. 295(5559): p. 1526-8.

13. Wartenberg, M., et al., Regulation of the multidrug resistance transporter Pglycoprotein in multicellular tumor spheroids by hypoxia-inducible factor (HIF-1) and reactive oxygen species. FASEB J, 2003. 17(3): p. 503-5. 
14. Comerford, K.M., et al., Hypoxia-inducible factor-1-dependent regulation of the multidrug resistance (MDR1) gene. Cancer Res, 2002. 62(12): p. 3387-94.

15. Wilson, W.R. and M.P. Hay, Targeting hypoxia in cancer therapy. Nat Rev Cancer, 2011. 11(6): p. 393-410.

16. Huang, L.E., et al., Regulation of hypoxia-inducible factor lalpha is mediated by an O2-dependent degradation domain via the ubiquitin-proteasome pathway. Proc Natl Acad Sci U S A, 1998. 95(14): p. 7987-92.

17. Salceda, S. and J. Caro, Hypoxia-inducible factor lalpha (HIF-1alpha) protein is rapidly degraded by the ubiquitin-proteasome system under normoxic conditions. Its stabilization by hypoxia depends on redox-induced changes. J Biol Chem, 1997. 272(36): p. 22642-7.

18. Ohh, M., Ubiquitin pathway in VHL cancer syndrome. Neoplasia, 2006. 8(8): p. 623-9.

19. Bruick, R.K. and S.L. McKnight, A conserved family of prolyl-4-hydroxylases that modify HIF. Science, 2001. 294(5545): p. 1337-40.

20. Epstein, A.C., et al., C. elegans EGL-9 and mammalian homologs define a family of dioxygenases that regulate HIF by prolyl hydroxylation. Cell, 2001. 107(1): p. 43-54.

21. Chi, J.T., et al., Gene expression programs in response to hypoxia: cell type specificity and prognostic significance in human cancers. PLoS Med, 2006. 3(3): p. e47.

22. Sowter, H.M., et al., Predominant role of hypoxia-inducible transcription factor (Hif)-1alpha versus Hif-2alpha in regulation of the transcriptional response to hypoxia. Cancer Res, 2003. 63(19): p. 6130-4.

23. Vengellur, A., et al., Gene expression profiling of the hypoxia signaling pathway in hypoxia-inducible factor lalpha null mouse embryonic fibroblasts. Gene Expr, 2003. 11(3-4): p. 181-97.

24. Semenza, G.L., Targeting HIF-1 for cancer therapy. Nat Rev Cancer, 2003. 3(10): p. 721-32.

25. Semenza, G.L., Defining the role of hypoxia-inducible factor 1 in cancer biology and therapeutics. Oncogene, 2010. 29(5): p. 625-34.

26. Stiehl, D.P., et al., Non-canonical HIF-2alpha function drives autonomous breast cancer cell growth via an AREG-EGFR/ErbB4 autocrine loop. Oncogene, 2011. 
27. Pocock, R., Invited review: decoding the microRNA response to hypoxia. Pflugers Arch, 2011. 461(3): p. 307-15.

28. Huang, X., et al., Hypoxia-inducible mir-210 regulates normoxic gene expression involved in tumor initiation. Mol Cell, 2009. 35(6): p. 856-67.

29. Camps, C., et al., hsa-miR-210 Is induced by hypoxia and is an independent prognostic factor in breast cancer. Clin Cancer Res, 2008. 14(5): p. 1340-8.

30. Wenger, R.H., et al., Nucleotide sequence, chromosomal assignment and mRNA expression of mouse hypoxia-inducible factor-1 alpha. Biochem Biophys Res Commun, 1996. 223(1): p. 54-9.

31. Wiener, C.M., G. Booth, and G.L. Semenza, In vivo expression of $m R N A s$ encoding hypoxia-inducible factor 1. Biochem Biophys Res Commun, 1996. 225(2): p. 485-8.

32. Ema, M., et al., A novel bHLH-PAS factor with close sequence similarity to hypoxia-inducible factor 1alpha regulates the VEGF expression and is potentially involved in lung and vascular development. Proc Natl Acad Sci U S A, 1997. 94(9): p. 4273-8.

33. Jain, S., et al., Expression of ARNT, ARNT2, HIF1 alpha, HIF2 alpha and Ah receptor $m R N A s$ in the developing mouse. Mechanisms of Development, 1998. 73(1): p. 117-23.

34. Hogenesch, J.B., et al., Characterization of a subset of the basic-helix-loop-helixPAS superfamily that interacts with components of the dioxin signaling pathway. $\mathrm{J}$ Biol Chem, 1997. 272(13): p. 8581-93.

35. Flamme, I., et al., HRF, a putative basic helix-loop-helix-PAS-domain transcription factor is closely related to hypoxia-inducible factor-1 alpha and developmentally expressed in blood vessels. Mech Dev, 1997. 63(1): p. 51-60.

36. Tian, H., et al., The hypoxia-responsive transcription factor EPASI is essential for catecholamine homeostasis and protection against heart failure during embryonic development. Genes Dev, 1998. 12(21): p. 3320-4.

37. Tian, H., S.L. McKnight, and D.W. Russell, Endothelial PAS domain protein 1 (EPAS1), a transcription factor selectively expressed in endothelial cells. Genes Dev, 1997. 11(1): p. 72-82.

38. Kozak, K.R., B. Abbott, and O. Hankinson, ARNT-deficient mice and placental differentiation. Dev Biol, 1997. 191(2): p. 297-305. 
39. Maltepe, E., et al., Abnormal angiogenesis and responses to glucose and oxygen deprivation in mice lacking the protein ARNT. Nature, 1997. 386(6623): p. 403-7.

40. Iyer, N.V., et al., Cellular and developmental control of O2 homeostasis by hypoxia-inducible factor 1 alpha. Genes Dev, 1998. 12(2): p. 149-62.

41. Ryan, H.E., J. Lo, and R.S. Johnson, HIF-1 alpha is required for solid tumor formation and embryonic vascularization. Embo J, 1998. 17(11): p. 3005-15.

42. Peng, J., et al., The transcription factor EPAS-1/hypoxia-inducible factor 2alpha plays an important role in vascular remodeling. Proc Natl Acad Sci, 2000. 97: p. 8386-91.

43. Compernolle, V., et al., Loss of HIF-2alpha and inhibition of VEGF impair fetal lung maturation, whereas treatment with VEGF prevents fatal respiratory distress in premature mice. Nat Med, 2002. 8(7): p. 702-10.

44. Doedens, A. and R.S. Johnson, Transgenic models to understand hypoxiainducible factor function. Methods Enzymol, 2007. 435: p. 87-105.

45. Boutin, A.T., et al., Epidermal sensing of oxygen is essential for systemic hypoxic response. Cell, 2008. 133: p. 223-234.

46. Cramer, T. and R.S. Johnson, A novel role for the hypoxia inducible transcription factor HIF-1alpha: critical regulation of inflammatory cell function. Cell Cycle, 2003. 2(3): p. 192-3.

47. Richardson, R.S., et al., Myoglobin O2 desaturation during exercise. Evidence of limited O2 transport. J Clin Invest, 1995. 96(4): p. 1916-26.

48. Gruber, M., et al., Acute postnatal ablation of Hif-2alpha results in anemia. Proc Natl Acad Sci U S A, 2007. 104(7): p. 2301-6.

49. Ryan, H.E., et al., Hypoxia-inducible factor-1alpha is a positive factor in solid tumor growth. Cancer Res, 2000. 60(15): p. 4010-5.

50. Seagroves, T.N., et al., HIF lalpha is a critical regulator of secretory differentiation and activation, but not vascular expansion, in the mouse mammary gland. Development, 2003. 130(8): p. 1713-24.

51. Elson, D.A., et al., Induction of hypervascularity without leakage or inflammation in transgenic mice overexpressing hypoxia-inducible factor-lalpha. Genes Dev, 2001. 15(19): p. 2520-32. 
52. Oosthuyse, B., et al., Deletion of the hypoxia-response element in the vascular endothelial growth factor promoter causes motor neuron degeneration. Nat Genet, 2001. 28(2): p. 131-8.

53. Huang, Y., et al., Cardiac myocyte-specific HIF-lalpha deletion alters vascularization, energy availability, calcium flux, and contractility in the normoxic heart. FASEB J, 2004. 18(10): p. 1138-40.

54. Karhausen, J., et al., Epithelial hypoxia-inducible factor-1 is protective in murine experimental colitis. J Clin Invest, 2004. 114(8): p. 1098-106.

55. Liao, D. and R.S. Johnson, Hypoxia: a key regulator of angiogenesis in cancer. Cancer Metastasis Rev, 2007. 26(2): p. 281-90.

56. Lee, K., et al., Anthracycline chemotherapy inhibits HIF-1 transcriptional activity and tumor-induced mobilization of circulating angiogenic cells. Proc Natl Acad Sci U S A, 2009. 106(7): p. 2353-8.

57. Lee, K., et al., Acriflavine inhibits HIF-1 dimerization, tumor growth, and vascularization. Proc Natl Acad Sci U S A, 2009. 106(42): p. 17910-5.

58. Barnhart, B.C. and M.C. Simon, Metastasis and stem cell pathways. Cancer Metastasis Rev, 2007. 26(2): p. 261-71.

59. Suda, T., K. Takubo, and G.L. Semenza, Metabolic regulation of hematopoietic stem cells in the hypoxic niche. Cell Stem Cell, 2011. 9(4): p. 298-310.

60. Wang, Y., et al., Targeting HIF lalpha Eliminates Cancer Stem Cells in Hematological Malignancies. Cell Stem Cell, 2011. 8(4): p. 399-411.

61. Luo, W., et al., Pyruvate kinase M2 is a PHD3-stimulated coactivator for hypoxia-inducible factor 1. Cell, 2011. 145(5): p. 732-44.

62. Zhang, H., et al., HIF-1 inhibits mitochondrial biogenesis and cellular respiration in VHL-deficient renal cell carcinoma by repression of $C-M Y C$ activity. Cancer Cell, 2007. 11(5): p. 407-20.

63. Franovic, A., et al., Translational up-regulation of the EGFR by tumor hypoxia provides a nonmutational explanation for its overexpression in human cancer. Proc Natl Acad Sci U S A, 2007. 104(32): p. 13092-7.

64. Esteban, M.A., et al., Regulation of E-cadherin expression by VHL and hypoxiainducible factor. Cancer Res, 2006. 66(7): p. 3567-75. 
65. Krishnamachary, B., et al., Hypoxia-inducible factor-1-dependent repression of E-cadherin in von Hippel-Lindau tumor suppressor-null renal cell carcinoma mediated by TCF3, ZFHX1A, and ZFHX1B. Cancer Res, 2006. 66(5): p. 2725-31.

66. Wu, M.Z., et al., Interplay between HDAC3 and WDR5 is essential for hypoxiainduced epithelial-mesenchymal transition. Mol Cell, 2011. 43(5): p. 811-22.

67. Mak, P., et al., ERbeta impedes prostate cancer EMT by destabilizing HIF-1alpha and inhibiting VEGF-mediated snail nuclear localization: implications for Gleason grading. Cancer Cell, 2010. 17(4): p. 319-32.

68. Krishnamachary, B. and G.L. Semenza, Analysis of hypoxia-inducible factor lalpha expression and its effects on invasion and metastasis. Methods Enzymol, 2007. 435: p. 347-54.

69. Sullivan, R. and C.H. Graham, Hypoxia-driven selection of the metastatic phenotype. Cancer Metastasis Rev, 2007. 26(2): p. 319-31.

70. Erler, J.T. and A.J. Giaccia, Lysyl oxidase mediates hypoxic control of metastasis. Cancer Res, 2006. 66(21): p. 10238-41.

71. Wong, C.C., et al., Hypoxia-inducible factor 1 is a master regulator of breast cancer metastatic niche formation. Proc Natl Acad Sci U S A, 2011. 108(39): p. 16369-74.

72. Zhang, H., et al., HIF-1-dependent expression of angiopoietin-like 4 and L1CAM mediates vascular metastasis of hypoxic breast cancer cells to the lungs. Oncogene, 2011.

73. Moeller, B.J., R.A. Richardson, and M.W. Dewhirst, Hypoxia and radiotherapy: opportunities for improved outcomes in cancer treatment. Cancer Metastasis Rev, 2007. 26(2): p. 241-8.

74. Rohwer, N. and T. Cramer, Hypoxia-mediated drug resistance: novel insights on the functional interaction of HIFs and cell death pathways. Drug Resist Updat, 2011. 14(3): p. 191-201.

75. Talks, K.L., et al., The expression and distribution of the hypoxia-inducible factors HIF- Ialpha and HIF-2alpha in normal human tissues, cancers, and tumor-associated macrophages. Am J Pathol, 2000. 157(2): p. 411-21.

76. Zhong, H., et al., Overexpression of hypoxia-inducible factor 1alpha in common human cancers and their metastases. Cancer Research, 1999. 59(22): p. 5830-5.

77. Zhong, H., et al., Overexpression of hypoxia-inducible factor 1a in common human cancers and their metastases. Cancer Res, 1999. 59: p. 5830-35. 
78. Harris, A.L., Hypoxia--a key regulatory factor in tumour growth. Nat Rev Cancer, 2002. 2(1): p. 38-47.

79. Maxwell, P.H., et al., Hypoxia-inducible factor-1 modulates gene expression in solid tumors and influences both angiogenesis and tumor growth. Proc Natl Acad Sci, 1997. 94: p. 8104-09.

80. Laughner, E., et al., HER2 (neu) signaling increases the rate of hypoxia-inducible factor lalpha (HIF-1alpha) synthesis: novel mechanism for HIF-1-mediated vascular endothelial growth factor expression. Mol Cell Biol, 2001. 21(12): p. 3995-4004.

81. Ravi, R., et al., Regulation of tumor angiogenesis by p53-induced degradation of hypoxia-inducible factor lalpha. Genes Dev, 2000. 14(1): p. 34-44.

82. Zundel, W., et al., Loss of PTEN facilitates HIF-1 mediated gene expression. Genes and Dev, 2000. 14: p. 391-396.

83. Vaupel, P., M. Hockel, and A. Mayer, Detection and characterization of tumor hypoxia using pO2 histography. Antioxid Redox Signal, 2007. 9(8): p. 1221-35.

84. Bos, R., et al., Levels of hypoxia-inducible factor-1 a during breast carcinogenesis. J. Natl. Cancer Instit., 2001. 93(4): p. 309-314.

85. Dales, J.P., et al., Overexpression of hypoxia-inducible factor HIF-1alpha predicts early relapse in breast cancer: retrospective study in a series of 745 patients. Int J Cancer, 2005. 116(5): p. 734-9.

86. Perou, C.M., et al., Molecular portraits of human breast tumours. Nature, 2000. 406(6797): p. 747-52.

87. Sorlie, T., et al., Gene expression patterns of breast carcinomas distinguish tumor subclasses with clinical implications. Proc Natl Acad Sci U S A, 2001. 98(19): p. 10869-74.

88. Foulkes, W.D., I.E. Smith, and J.S. Reis-Filho, Triple-negative breast cancer. N Engl J Med, 2010. 363(20): p. 1938-48.

89. Lehmann, B.D., et al., Identification of human triple-negative breast cancer subtypes and preclinical models for selection of targeted therapies. J Clin Invest, 2011. 121(7): p. 2750-67.

90. Whelan, K.A., et al., The Oncogene HER2/neu (ERBB2) Requires the Hypoxiainducible Factor HIF-1 for Mammary Tumor Growth and Anoikis Resistance. J Biol Chem, 2013. 288(22): p. 15865-77. 
91. Ignatiadis, M. and C. Sotiriou, Luminal breast cancer: from biology to treatment. Nat Rev Clin Oncol, 2013.

92. Gatza, M.L., et al., Analysis of tumor environmental response and oncogenic pathway activation identifies distinct basal and luminal features in HER2-related breast tumor subtypes. Breast Cancer Res, 2011. 13(3): p. R62.

93. Comprehensive molecular portraits of human breast tumours. Nature, 2012. 490(7418): p. 61-70.

94. Gordan, J.D., et al., HIF-2alpha promotes hypoxic cell proliferation by enhancing c-myc transcriptional activity. Cancer Cell, 2007. 11(4): p. 335-47.

95. Helczynska, K., et al., Hypoxia-inducible factor-2alpha correlates to distant recurrence and poor outcome in invasive breast cancer. Cancer Res, 2008. 68(22): p. 9212-20.

96. Gort, E.H., et al., Hypoxic regulation of metastasis via hypoxia-inducible factors. Curr Mol Med, 2008. 8(1): p. 60-7.

97. Krishnamachary, B., et al., Regulation of colon carcinoma cell invasion by hypoxia-inducible factor 1. Cancer Res, 2003. 63(5): p. 1138-43.

98. Gupta, G.P., et al., Mediators of vascular remodelling co-opted for sequential steps in lung metastasis. Nature, 2007. 446(7137): p. 765-70.

99. Martens, L.K., et al., Hypoxia-inducible factor-1 (HIF-1) is a transcriptional activator of the TrkB neurotrophin receptor gene. J Biol Chem, 2007. 282(19): p. 14379-88.

100. Douma, S., et al., Suppression of anoikis and induction of metastasis by the neurotrophic receptor TrkB. Nature, 2004. 430(7003): p. 1034-9.

101. Lu, X. and Y. Kang, Hypoxia and hypoxia-inducible factors: master regulators of metastasis. Clin Cancer Res, 2010. 16(24): p. 5928-35.

102. Brown, J.M., Exploiting the hypoxic cancer cell: mechanisms and therapeutic strategies. Mol Med Today, 2000. 6(4): p. 157-62.

103. Giaccia, A., B.G. Siim, and R.S. Johnson, HIF-1 as a target for drug development. Nat Rev Drug Discov, 2003. 2(10): p. 803-11.

104. Zhang, H., et al., Digoxin and other cardiac glycosides inhibit HIF-1alpha synthesis and block tumor growth. Proc Natl Acad Sci U S A, 2008. 105(50): p. 19579-86. 
105. Chun, Y.S., et al., Inhibitory effect of YC-1 on the hypoxic induction of erythropoietin and vascular endothelial growth factor in Hep3B cells. Biochem Pharmacol, 2001. 61(8): p. 947-54.

106. Shin, D.H., et al., Preclinical evaluation of YC-1, a HIF inhibitor, for the prevention of tumor spreading. Cancer Lett, 2007. 255(1): p. 107-16.

107. Welsh, S., et al., Antitumor activity and pharmacodynamic properties of $P X-478$, an inhibitor of hypoxia-inducible factor-1alpha. Mol Cancer Ther, 2004. 3(3): $\mathrm{p}$. 233-44.

108. Hudson, C.C., et al., Regulation of hypoxia-inducible factor lalpha expression and function by the mammalian target of rapamycin. Mol Cell Biol, 2002. 22(20): p. 7004-14.

109. Reya, T., et al., Stem cells, cancer, and cancer stem cells. Nature, 2001. 414(6859): p. 105-11.

110. Li, F., et al., Beyond tumorigenesis: cancer stem cells in metastasis. Cell Res, 2007. 17(1): p. 3-14.

111. Mohyeldin, A., T. Garzon-Muvdi, and A. Quinones-Hinojosa, Oxygen in stem cell biology: a critical component of the stem cell niche. Cell Stem Cell, 2010. 7(2): p. 150-61.

112. Takubo, K., et al., Regulation of the HIF-1alpha level is essential for hematopoietic stem cells. Cell Stem Cell, 2010. 7(3): p. 391-402.

113. Conley, S.J., et al., Antiangiogenic agents increase breast cancer stem cells via the generation of tumor hypoxia. Proc Natl Acad Sci U S A, 2012. 109(8): p. 2784-9.

114. Lacerda, L., L. Pusztai, and W.A. Woodward, The role of tumor initiating cells in drug resistance of breast cancer: Implications for future therapeutic approaches. Drug resistance updates : reviews and commentaries in antimicrobial and anticancer chemotherapy, 2010. 13(4-5): p. 99-108.

115. Li, Z., et al., Hypoxia-inducible factors regulate tumorigenic capacity of glioma stem cells. Cancer Cell, 2009. 15(6): p. 501-13.

116. Matsumoto, K., et al., mTOR signal and hypoxia-inducible factor-1 alpha regulate CD133 expression in cancer cells. Cancer research, 2009. 69(18): p. 7160-4.

117. Soeda, A., et al., Hypoxia promotes expansion of the CD133-positive glioma stem cells through activation of HIF-1alpha. Oncogene, 2009. 28(45): p. 3949-59. 
118. Kouros-Mehr, H., et al., GATA-3 links tumor differentiation and dissemination in a luminal breast cancer model. Cancer Cell, 2008. 13(2): p. 141-52.

119. Vaillant, F., et al., The mammary progenitor marker CD61/beta3 integrin identifies cancer stem cells in mouse models of mammary tumorigenesis. Cancer Res, 2008. 68(19): p. 7711-7.

120. Wright, M.H., et al., Brcal breast tumors contain distinct CD44+/CD24- and CD133+ cells with cancer stem cell characteristics. Breast Cancer Res, 2008. 10(1): p. R10.

121. Zhang, M., et al., Identification of tumor-initiating cells in a p53-null mouse model of breast cancer. Cancer research, 2008. 68(12): p. 4674-82.

122. Charafe-Jauffret, E., et al., Breast cancer cell lines contain functional cancer stem cells with metastatic capacity and a distinct molecular signature. Cancer Res, 2009. 69(4): p. 1302-13.

123. Liu, H., et al., Cancer stem cells from human breast tumors are involved in spontaneous metastases in orthotopic mouse models. Proceedings of the National Academy of Sciences of the United States of America, 2010. 107(42): p. 1811520.

124. Lin, E.Y., et al., Progression to malignancy in the polyoma middle T oncoprotein mouse breast cancer model provides a reliable model for human diseases. Am J Pathol, 2003. 163(5): p. 2113-26.

125. Herschkowitz, J.I., et al., Identification of conserved gene expression features between murine mammary carcinoma models and human breast tumors. Genome Biol, 2007. 8(5): p. R76.

126. Liao, D., et al., Hypoxia-inducible factor-1alpha is a key regulator of metastasis in a transgenic model of cancer initiation and progression. Cancer Res, 2007. 67(2): p. 563-72.

127. Schwab, L.P., et al., Hypoxia-inducible factor lalpha promotes primary tumor growth and tumor-initiating cell activity in breast cancer. Breast Cancer Res, 2012. 14(1): p. R6.

128. Varticovski, L., et al., Accelerated preclinical testing using transplanted tumors from genetically engineered mouse breast cancer models. Clin Cancer Res, 2007. 13(7): p. 2168-77.

129. Medina, D. and H. Thompson, A Comparison of the Salient Features of Mouse, Rat, and Human Mammary Tumorigenesis, in Methods in Mammary Gland Biology and Breast Cancer Research. 2000, Springer US. p. 31-36. 
130. Wagner, K.U., Models of breast cancer: quo vadis, animal modeling? Breast Cancer Res, 2004. 6(1): p. 31-8.

131. Platet, N., et al., Influence of oxygen tension on CD133 phenotype in human glioma cell cultures. Cancer Lett, 2007. 258(2): p. 286-90.

132. DeRose, Y.S., et al., Tumor grafts derived from women with breast cancer authentically reflect tumor pathology, growth, metastasis and disease outcomes. Nat Med, 2011. 17(11): p. 1514-20.

133. Zhang, X., et al., A renewable tissue resource of phenotypically stable, biologically and ethnically diverse, patient-derived human breast cancer xenograft models. Cancer research, 2013. 73(15): p. 4885-97.

134. Kabos, P., et al., Patient-derived luminal breast cancer xenografts retain hormone receptor heterogeneity and help define unique estrogen-dependent gene signatures. Breast cancer research and treatment, 2012. 135(2): p. 415-32.

135. Landis, M.D., et al., Patient-derived breast tumor xenografts facilitating personalized cancer therapy. Breast cancer research : BCR, 2013. 15(1): p. 201.

136. Haase, V.H., et al., Vascular tumors in livers with targeted inactivation of the von Hippel-Lindau tumor suppressor. Proceedings of the National Academy of Sciences of the United States of America, 2001. V98(N4): p. 1583-1588.

137. Wagner, K.U., et al., Spatial and temporal expression of the Cre gene under the control of the MMTV-LTR in different lines of transgenic mice. Transgenic Res, 2001. 10(6): p. 545-53.

138. Wagner, K.U., et al., Cre-mediated gene deletion in the mammary gland. Nucleic Acids Res, 1997. 25(21): p. 4323-30.

139. Seagroves, T.N., et al., C/EBPbeta, but not C/EBPalpha, is essential for ductal morphogenesis, lobuloalveolar proliferation, and functional differentiation in the mouse mammary gland. Genes Dev, 1998. 12(12): p. 1917-28.

140. Seagroves, T.N., et al., C/EBPbeta (CCAAT/enhancer binding protein) controls cell fate determination during mammary gland development. Mol Endocrinol, 2000. 14(3): p. 359-68.

141. Corless, C.L., et al., Immunostaining of the von Hippel-Lindau gene product in normal and neoplastic human tissues. Hum Pathol, 1997. 28(4): p. 459-64.

142. Boulanger, C.A., K.U. Wagner, and G.H. Smith, Parity-induced mouse mammary epithelial cells are pluripotent, self-renewing and sensitive to TGF-beta1 expression. Oncogene, 2005. 24(4): p. 552-60. 
143. Seagroves, T.N., et al., VHL deletion impairs mammary alveologenesis but is not sufficient for mammary tumorigenesis. Am J Pathol, 2010. 176(5): p. 2269-82.

144. McManaman, J.L. and M.C. Neville, Mammary physiology and milk secretion. Adv Drug Deliv Rev, 2003. 55(5): p. 629-41.

145. Anderson, S.M., et al., Key stages in mammary gland development. Secretory activation in the mammary gland: it's not just about milk protein synthesis! Breast Cancer Res, 2007. 9(1): p. 204.

146. Anderson, L.H., et al., Stem cell marker prominin-1 regulates branching morphogenesis, but not regenerative capacity, in the mammary gland. Dev Dyn, 2011. 240(3): p. 674-81.

147. Rosen, J.M., S.L. Wyszomierski, and D. Hadsell, Regulation of milk protein gene expression. Annu Rev Nutr, 1999. 19: p. 407-36.

148. Arias-Stella, J., The Arias-Stella reaction: facts and fancies four decades after. Adv Anat Pathol, 2002. 9(1): p. 12-23.

149. Henry, M.D., et al., Parity-induced mammary epithelial cells facilitate tumorigenesis in MMTV-neu transgenic mice. Oncogene, 2004. 23(41): p. 6980-5.

150. Landis, M.D., et al., Sustained trophism of the mammary gland is sufficient to accelerate and synchronize development of ErbB2/Neu-induced tumors. Oncogene, 2006. 25(23): p. 3325-34.

151. Roland, C.L., et al., Cytokine levels correlate with immune cell infiltration after anti-VEGF therapy in preclinical mouse models of breast cancer. PLoS One, 2009. 4(11): p. e7669.

152. Lantzsch, T., et al., Expression of the von Hippel-Lindau gene protein in breast cancer tissue. Gynecol Oncol, 2002. 84(1): p. 186-7.

153. Zia, M.K., et al., The expression of the von Hippel-Lindau gene product and its impact on invasiveness of human breast cancer cells. Int J Mol Med, 2007. 20(4): p. 605-11.

154. Appelhoff, R.J., et al., Differential function of the prolyl hydroxylases PHD1, PHD2, and PHD3 in the regulation of hypoxia-inducible factor. J Biol Chem, 2004. 279(37): p. 38458-65.

155. Wagner, K.U., et al., An adjunct mammary epithelial cell population in parous females: its role in functional adaptation and tissue renewal. Development, 2002. 129(6): p. 1377-86. 
156. Keith, B. and M.C. Simon, Hypoxia-inducible factors, stem cells, and cancer. Cell, 2007. 129(3): p. 465-72.

157. Hu, C.J., et al., Differential regulation of the transcriptional activities of hypoxiainducible factor 1 alpha (HIF-1alpha) and HIF-2alpha in stem cells. Mol Cell Biol, 2006. 26(9): p. 3514-26.

158. Gustafsson, M.V., et al., Hypoxia requires notch signaling to maintain the undifferentiated cell state. Dev Cell, 2005. 9(5): p. 617-28.

159. Kaidi, A., A.C. Williams, and C. Paraskeva, Interaction between beta-catenin and HIF-1 promotes cellular adaptation to hypoxia. Nat Cell Biol, 2007. 9(2): p. 2107.

160. Brown, J.M. and A.J. Giaccia, The unique physiology of solid tumors: opportunities (and problems) for cancer therapy. Cancer Research, 1998. 58(7): p. 1408-16.

161. Zhang, M. and J.M. Rosen, Stem cells in the etiology and treatment of cancer. Curr Opin Genet Dev, 2006. 16(1): p. 60-4.

162. Rich, J.N., Cancer stem cells in radiation resistance. Cancer Res, 2007. 67(19): p. 8980-4.

163. Rich, J.N. and S. Bao, Chemotherapy and cancer stem cells. Cell Stem Cell, 2007. 1(4): p. 353-5.

164. Dontu, G., et al., Role of Notch signaling in cell-fate determination of human mammary stem/progenitor cells. Breast Cancer Res, 2004. 6(6): p. R605-15.

165. Shackleton, M., et al., Generation of a functional mammary gland from a single stem cell. Nature, 2006. 439(7072): p. 84-8.

166. Stingl, J., et al., Purification and unique properties of mammary epithelial stem cells. Nature, 2006. 439(7079): p. 993-7.

167. Matulka, L.A., A.A. Triplett, and K.U. Wagner, Parity-induced mammary epithelial cells are multipotent and express cell surface markers associated with stem cells. Dev Biol, 2007. 303(1): p. 29-44.

168. Creamer, B.A., A.A. Triplett, and K.U. Wagner, Longitudinal analysis of mammogenesis using a novel tetracycline-inducible mouse model and in vivo imaging. Genesis, 2009. 47(4): p. 234-45. 
169. Sansone, P., et al., p66Shc/Notch-3 interplay controls self-renewal and hypoxia survival in human stem/progenitor cells of the mammary gland expanded in vitro as mammospheres. Stem Cells, 2007. 25(3): p. 807-15.

170. Hovey, R.C., et al., Transcriptional regulation of vascular endothelial growth factor expression in epithelial and stromal cells during mouse mammary gland development. Mol Endo, 2001. 15(5): p. 819-31.

171. Pepper, M.S., et al., Vascular endothelial growth factor (VEGF)-C synergizes with basic fibroblast growth factor and VEGF in the induction of angiogenesis in vitro and alters endothelial cell extracellular proteolytic activity. J Cell Physiol, 1998. 177(3): p. 439-52.

172. Rossiter, H., et al., Inactivation of VEGF in mammary gland epithelium severely compromises mammary gland development and function. FASEB J, 2007. 21(14): p. 3994-4004.

173. Maynard, M.A. and M. Ohh, Von Hippel-Lindau tumor suppressor protein and hypoxia-inducible factor in kidney cancer. Am J Nephrol, 2004. 24(1): p. 1-13.

174. Aprelikova, O., et al., Role of ETS transcription factors in the hypoxia-inducible factor-2 target gene selection. Cancer Res, 2006. 66(11): p. 5641-7.

175. Blancher, C., et al., Relationship of hypoxia-inducible factor (HIF)-1alpha and HIF-2alpha expression to vascular endothelial growth factor induction and hypoxia survival in human breast cancer cell lines. Cancer Res, 2000. 60(24): p. 7106-13.

176. Czyzyk-Krzeska, M.F. and J. Meller, von Hippel-Lindau tumor suppressor: not only HIF's executioner. Trends Mol Med, 2004. 10(4): p. 146-9.

177. Frew, I.J. and W. Krek, Multitasking by pVHL in tumour suppression. Curr Opin Cell Biol, 2007. 19(6): p. 685-90.

178. Lolkema, M.P., et al., The von Hippel-Lindau tumour suppressor interacts with microtubules through kinesin-2. FEBS Lett, 2007. 581(24): p. 4571-6.

179. Stickle, N.H., et al., pVHL modification by NEDD8 is required for fibronectin matrix assembly and suppression of tumor development. Mol Cell Biol, 2004. 24(8): p. 3251-61.

180. Tang, N., et al., $p V H L$ function is essential for endothelial extracellular matrix deposition. Mol Cell Biol, 2006. 26(7): p. 2519-30. 
181. Chitalia, V.C., et al., Jade-1 inhibits Wnt signalling by ubiquitylating beta-catenin and mediates Wnt pathway inhibition by pVHL. Nat Cell Biol, 2008. 10(10): p. 1208-16.

182. Young, A.P., et al., VHL loss actuates a HIF-independent senescence programme mediated by $R b$ and $p 400$. Nat Cell Biol, 2008. 10(3): p. 361-9.

183. Wagner, K.U., et al., Impaired alveologenesis and maintenance of secretory mammary epithelial cells in Jak2 conditional knockout mice. Mol Cell Biol, 2004. 24(12): p. 5510-20.

184. Cui, Y., et al., Inactivation of Stat5 in mouse mammary epithelium during pregnancy reveals distinct functions in cell proliferation, survival, and differentiation. Mol Cell Biol, 2004. 24(18): p. 8037-47.

185. Joung, Y.H., et al., Hypoxia activates the cyclin D1 promoter via the Jak2/STAT5b pathway in breast cancer cells. Exp Mol Med, 2005. 37(4): p. 35364.

186. Hunter, K., D.R. Welch, and E.T. Liu, Genetic background is an important determinant of metastatic potential. Nat Genet, 2003. 34(1): p. 23-4; author reply 25.

187. Rijnkels, M. and J.M. Rosen, Adenovirus-Cre-mediated recombination in mammary epithelial early progenitor cells. J Cell Sci, 2001. 114(Pt 17): p. 314753.

188. Dontu, G., et al., In vitro propagation and transcriptional profiling of human mammary stem/progenitor cells. Genes Dev, 2003. 17(10): p. 1253-70.

189. $\mathrm{Hu}, \mathrm{Y}$. and G.K. Smyth, ELDA: extreme limiting dilution analysis for comparing depleted and enriched populations in stem cell and other assays. J Immunol Methods, 2009. 347(1-2): p. 70-8.

190. Wang, G.L., et al., Hypoxia-inducible factor 1 is a basic-helix-loop-helix-PAS heterodimer regulated by cellular $0_{2}$ tension. Proc Natl Acad Sci, 1995. 92: p. 5510-4.

191. Ginouves, A., et al., PHDs overactivation during chronic hypoxia "desensitizes" HIFalpha and protects cells from necrosis. Proc Natl Acad Sci U S A, 2008. 105(12): p. 4745-50.

192. Peng, X.H., et al., Cross-talk between epidermal growth factor receptor and hypoxia-inducible factor-1 alpha signal pathways increases resistance to apoptosis by up-regulating survivin gene expression. J Biol Chem, 2006. 281(36): p. 25903-14. 
193. Seagroves, T.N., et al., Transcription factor HIF-1 is a necessary mediator of the pasteur effect in mammalian cells. Mol Cell Biol, 2001. 21(10): p. 3436-44.

194. Storci, G., et al., The basal-like breast carcinoma phenotype is regulated by SLUG gene expression. J Pathol, 2007.

195. Sahlgren, C., et al., Notch signaling mediates hypoxia-induced tumor cell migration and invasion. Proc Natl Acad Sci U S A, 2008.

196. Visbal, A.P., et al., Altered differentiation and paracrine stimulation of mammary epithelial cell proliferation by conditionally activated Smoothened. Dev Biol, 2011. 352(1): p. 116-27.

197. Bar, E.E., et al., Hypoxia increases the expression of stem-cell markers and promotes clonogenicity in glioblastoma neurospheres. The American journal of pathology, 2010. 177(3): p. 1491-502.

198. Chen, J., N. Imanaka, and J.D. Griffin, Hypoxia potentiates Notch signaling in breast cancer leading to decreased E-cadherin expression and increased cell migration and invasion. Br J Cancer, 2010. 102(2): p. 351-60.

199. Leong, K.G., et al., Jagged1-mediated Notch activation induces epithelial-tomesenchymal transition through Slug-induced repression of E-cadherin. J Exp Med, 2007. 204(12): p. 2935-48.

200. Lifsted, T., et al., Identification of inbred mouse strains harboring genetic modifiers of mammary tumor age of onset and metastatic progression. Int $\mathrm{J}$ Cancer, 1998. 77(4): p. 640-4.

201. Nielsen, T.O., et al., Immunohistochemical and clinical characterization of the basal-like subtype of invasive breast carcinoma. Clin Cancer Res, 2004. 10(16): p. 5367-74.

202. Yamamoto, Y., et al., Clinical significance of basal-like subtype in triple-negative breast cancer. Breast Cancer, 2009. 16(4): p. 260-7.

203. Cheang, M.C., et al., Basal-like breast cancer defined by five biomarkers has superior prognostic value than triple-negative phenotype. Clin Cancer Res, 2008. 14(5): p. 1368-76.

204. Sutton, L.M., et al., Intratumoral expression level of epidermal growth factor receptor and cytokeratin 5/6 is significantly associated with nodal and distant metastases in patients with basal-like triple-negative breast carcinoma. Am J Clin Pathol, 2010. 134(5): p. 782-7. 
205. El Guerrab, A., et al., Differential Impact of EGFR-Targeted Therapies on Hypoxia Responses: Implications for Treatment Sensitivity in Triple-Negative Metastatic Breast Cancer. PLoS One, 2011. 6(9): p. e25080.

206. Sleeman, K.E., et al., Dissociation of estrogen receptor expression and in vivo stem cell activity in the mammary gland. J Cell Biol, 2007. 176(1): p. 19-26.

207. Cooper, C., et al., Intermittent hypoxia induces proteasome-dependent downregulation of estrogen receptor alpha in human breast carcinoma. Clin Cancer Res, 2004. 10(24): p. 8720-7.

208. Stoner, M., et al., Hypoxia induces proteasome-dependent degradation of estrogen receptor alpha in ZR-75 breast cancer cells. Mol Endocrinol, 2002. 16(10): p. 2231-42.

209. Meyer, M.J., et al., CD44posCD49fhiCD133/2hi defines xenograft-initiating cells in estrogen receptor-negative breast cancer. Cancer Res, 2010. 70(11): p. 462433.

210. Lim, E., et al., Aberrant luminal progenitors as the candidate target population for basal tumor development in BRCA1 mutation carriers. Nat Med, 2009. 15(8): p. 907-13.

211. Molyneux, G., et al., BRCAl basal-like breast cancers originate from luminal epithelial progenitors and not from basal stem cells. Cell Stem Cell, 2010. 7(3): p. 403-17.

212. Proia, T.A., et al., Genetic predisposition directs breast cancer phenotype by dictating progenitor cell fate. Cell Stem Cell, 2011. 8(2): p. 149-63.

213. van der Groep, P., et al., High frequency of HIF-1alpha overexpression in BRCAI related breast cancer. Breast Cancer Res Treat, 2008. 111(3): p. 475-80.

214. Yan, M., et al., BRCA1 tumours correlate with a HIF-lalpha phenotype and have a poor prognosis through modulation of hydroxylase enzyme profile expression. Br J Cancer, 2009. 101(7): p. 1168-74.

215. Chaffer, C.L., et al., Normal and neoplastic nonstem cells can spontaneously convert to a stem-like state. Proc Natl Acad Sci U S A, 2011. 108(19): p. 7950-5.

216. Gupta, P.B., et al., Stochastic state transitions give rise to phenotypic equilibrium in populations of cancer cells. Cell, 2011. 146(4): p. 633-44.

217. Van Keymeulen, A., et al., Distinct stem cells contribute to mammary gland development and maintenance. Nature, 2011. 
218. Harrison, H., et al., Breast cancer stem cells: something out of notching? Cancer Res, 2010. 70(22): p. 8973-6.

219. Asselin-Labat, M.L., et al., Gata-3 negatively regulates the tumor-initiating capacity of mammary luminal progenitor cells and targets the putative tumor suppressor caspase-14. Mol Cell Biol, 2011.

220. Malanchi, I., et al., Interactions between cancer stem cells and their niche govern metastatic colonization. Nature, 2012. 481(7379): p. 85-9.

221. Grange, C., et al., Sca-1 identifies the tumor-initiating cells in mammary tumors of BALB-neuT transgenic mice. Neoplasia, 2008. 10(12): p. 1433-43.

222. Louie, E., et al., Identification of a stem-like cell population by exposing metastatic breast cancer cell lines to repetitive cycles of hypoxia and reoxygenation. Breast Cancer Res, 2010. 12(6): p. R94.

223. Krishnamachary, B., et al., Hypoxia regulates CD44 and its variant isoforms through HIF-1alpha in triple negative breast cancer. PLoS One, 2012. 7(8): p. e44078.

224. Thomas, S., et al., CD24 is an effector of HIF-1-driven primary tumor growth and metastasis. Cancer Res, 2012. 72(21): p. 5600-12.

225. Al-Hajj, M., et al., Prospective identification of tumorigenic breast cancer cells. Proc Natl Acad Sci U S A, 2003. 100(7): p. 3983-8.

226. Cho, R.W., et al., Isolation and molecular characterization of cancer stem cells in MMTV-Wnt-1 murine breast tumors. Stem Cells, 2008. 26(2): p. 364-71.

227. Grosse-Gehling, P., et al., CD133 as a biomarker for putative cancer stem cells in solid tumours: limitations, problems and challenges. J Pathol, 2013. 229(3): $\mathrm{p}$. $355-78$.

228. Visvader, J.E., Keeping abreast of the mammary epithelial hierarchy and breast tumorigenesis. Genes Dev, 2009. 23(22): p. 2563-77.

229. Keller, P.J., et al., Mapping the cellular and molecular heterogeneity of normal and malignant breast tissues and cultured cell lines. Breast Cancer Res, 2010. 12(5): p. R87.

230. Bon, G., et al., Involvement of alpha6beta4 integrin in the mechanisms that regulate breast cancer progression. Breast Cancer Res, 2007. 9(1): p. 203.

231. Friedrichs, K., et al., High expression level of alpha 6 integrin in human breast carcinoma is correlated with reduced survival. Cancer Res, 1995. 55(4): p. 901-6. 
232. Yoon, S.O., S. Shin, and A.M. Mercurio, Hypoxia stimulates carcinoma invasion by stabilizing microtubules and promoting the Rabl1 trafficking of the alpha6beta4 integrin. Cancer Res, 2005. 65(7): p. 2761-9.

233. Stiehl, D.P., et al., Non-canonical HIF-2alpha function drives autonomous breast cancer cell growth via an AREG-EGFR/ErbB4 autocrine loop. Oncogene, 2012. 31(18): p. 2283-97.

234. Hu, Z., et al., The molecular portraits of breast tumors are conserved across microarray platforms. BMC Genomics, 2006. 7: p. 96.

235. Cancer facts and figures, 2008. 2008.

236. van 't Veer, L.J., et al., Gene expression profiling predicts clinical outcome of breast cancer. Nature, 2002. 415: p. 530-6.

237. Wang, Y., et al., Integrin subunits alpha5 and alpha6 regulate cell cycle by modulating the chkl and Rb/E2F pathways to affect breast cancer metastasis. Mol Cancer, 2011. 10: p. 84.

238. Hendrix, M.J., et al., Experimental co-expression of vimentin and keratin intermediate filaments in human breast cancer cells results in phenotypic interconversion and increased invasive behavior. Am J Pathol, 1997. 150(2): $\mathrm{p}$. $483-95$.

239. Guo, W., et al., Beta 4 integrin amplifies ErbB2 signaling to promote mammary tumorigenesis. Cell, 2006. 126(3): p. 489-502.

240. Gerson, K.D., et al., Integrin beta4 regulates SPARC protein to promote invasion. J Biol Chem, 2012. 287(13): p. 9835-44.

241. Kim, J.W., et al., HIF-1-mediated expression of pyruvate dehydrogenase kinase: a metabolic switch required for cellular adaptation to hypoxia. Cell Metab, 2006. 3(3): p. 177-85.

242. Anan, K., et al., Vascular endothelial growth factor and platelet-derived growth factor are potential angiogenic and metastatic factors in human breast cancer. Surgery, 1996. 119(3): p. 333-9.

243. Ahmad, A. and I.R. Hart, Mechanisms of metastasis. Critical Reviews in Oncology/Hematology, 1997. 26(3): p. 163-73.

244. Koike, T., et al., Hypoxia induces adhesion molecules on cancer cells: A missing link between Warburg effect and induction of selectin-ligand carbohydrates. Proc Natl Acad Sci U S A, 2004. 101(21): p. 8132-7. 
245. Mishra, A., et al., Hypoxia stabilizes GAS6/Axl signaling in metastatic prostate cancer. Mol Cancer Res, 2012. 10(6): p. 703-12.

246. Xia, X., et al., Integrative analysis of HIF binding and transactivation reveals its role in maintaining histone methylation homeostasis. Proc Natl Acad Sci U S A, 2009. 106(11): p. 4260-5.

247. Asselin-Labat, M.L., et al., Gata-3 is an essential regulator of mammary-gland morphogenesis and luminal-cell differentiation. Nat Cell Biol, 2007. 9(2): p. 2019.

248. Fodde, R. and T. Brabletz, Wnt/beta-catenin signaling in cancer stemness and malignant behavior. Curr Opin Cell Biol, 2007. 19(2): p. 150-8.

249. Wallimann, T., M. Tokarska-Schlattner, and U. Schlattner, The creatine kinase system and pleiotropic effects of creatine. Amino Acids, 2011. 40(5): p. 1271-96.

250. Miller, K., J. Halow, and A.P. Koretsky, Phosphocreatine protects transgenic mouse liver expressing creatine kinase from hypoxia and ischemia. Am J Physiol, 1993. 265(6 Pt 1): p. C1544-51.

251. Wyss, M. and R. Kaddurah-Daouk, Creatine and creatinine metabolism. Physiol Rev, 2000. 80(3): p. 1107-213.

252. Mooney, S.M., et al., Creatine kinase brain overexpression protects colorectal cells from various metabolic and non-metabolic stresses. J Cell Biochem, 2011. 112(4): p. 1066-75.

253. Zarghami, N., et al., Creatine kinase BB isoenzyme levels in tumour cytosols and survival of breast cancer patients. Br J Cancer, 1996. 73(3): p. 386-90.

254. Wallimann, T. and W. Hemmer, Creatine kinase in non-muscle tissues and cells. Mol Cell Biochem, 1994. 133-134: p. 193-220.

255. Balasubramani, M., et al., Altered expression and localization of creatine kinase $B$, heterogeneous nuclear ribonucleoprotein $F$, and high mobility group box 1 protein in the nuclear matrix associated with colon cancer. Cancer Res, 2006. 66(2): p. 763-9.

256. Huddleston, H.G., et al., Clinical applications of microarray technology: creatine kinase $B$ is an up-regulated gene in epithelial ovarian cancer and shows promise as a serum marker. Gynecol Oncol, 2005. 96(1): p. 77-83.

257. Li, X.H., et al., Knockdown of creatine kinase B inhibits ovarian cancer progression by decreasing glycolysis. Int J Biochem Cell Biol, 2013. 45(5): p. 979-86. 
258. Kuiper, J.W., et al., Local ATP generation by brain-type creatine kinase (CK-B) facilitates cell motility. PLoS One, 2009. 4(3): p. e5030.

259. Martin, K.J., et al., Evaluation of creatine analogues as a new class of anticancer agents using freshly explanted human tumor cells. J Natl Cancer Inst, 1994. 86(8): p. $608-13$.

260. Annesley, T.M. and J.B. Walker, Cyclocreatine phosphate as a substitute for creatine phosphate in vertebrate tissues. Energistic considerations. Biochem Biophys Res Commun, 1977. 74(1): p. 185-90.

261. Lillie, J.W., et al., Cyclocreatine (1-carboxymethyl-2-iminoimidazolidine) inhibits growth of a broad spectrum of cancer cells derived from solid tumors. Cancer Res, 1993. 53(13): p. 3172-8.

262. Teicher, B.A., et al., Cyclocreatine in cancer chemotherapy. Cancer Chemother Pharmacol, 1995. 35(5): p. 411-6.

263. Mulvaney, P.T., et al., Cyclocreatine inhibits stimulated motility in tumor cells possessing creatine kinase. Int J Cancer, 1998. 78(1): p. 46-52.

264. Kurosawa, Y., et al., Cyclocreatine treatment improves cognition in mice with creatine transporter deficiency. J Clin Invest, 2012. 122(8): p. 2837-46.

265. Campeau, E., et al., A versatile viral system for expression and depletion of proteins in mammalian cells. PLoS One, 2009. 4(8): p. e6529.

266. Tonon, L., H. Touzet, and J.S. Varre, TFM-Explorer: mining cis-regulatory regions in genomes. Nucleic Acids Res, 2010. 38(Web Server issue): p. W286-92.

267. Wang, Y., et al., Gene-expression profiles to predict distant metastasis of lymphnode-negative primary breast cancer. Lancet, 2005. 365(9460): p. 671-9.

268. Bos, R., et al., Hypoxia-inducible factor-1alpha is associated with angiogenesis, and expression of $b F G F, P D G F-B B$, and EGFR in invasive breast cancer. Histopathology, 2005. 46(1): p. 31-6.

269. Chaturvedi, P., et al., Hypoxia-inducible factor-dependent breast cancermesenchymal stem cell bidirectional signaling promotes metastasis. J Clin Invest, 2013. 123(1): p. 189-205.

270. Goonewardene, T.I., H.M. Sowter, and A.L. Harris, Hypoxia-induced pathways in breast cancer. Microsc Res Tech, 2002. 59(1): p. 41-8. 
271. Gort, E.H., et al., Hypoxia-inducible factor-1alpha expression requires PI 3kinase activity and correlates with Akt1 phosphorylation in invasive breast carcinomas. Oncogene, 2006. 25(45): p. 6123-7.

272. Bergnes, G., et al., Creatine and phosphocreatine analogs: anticancer activity and enzymatic analysis. Oncol Res, 1996. 8(3): p. 121-30.

273. Keller, P.J., et al., Defining the cellular precursors to human breast cancer. Proc Natl Acad Sci U S A, 2012. 109(8): p. 2772-7.

274. Warburg, O., The Metabolism of Tumours. 1930, London: Constable \& Co., Ltd. 327.

275. Graeber, T.G., et al., Hypoxia-mediated selection of cells with diminished apoptotic potential in solid tumours [see comments]. Nature, 1996. 379(6560): $\mathrm{p}$. 88-91.

276. Semenza, G.L. and G.L. Wang, A nuclear factor induced by hypoxia via de novo protein synthesis binds to the human erythropoietin gene enhancer at a site required for transcriptional activation. Mol Cell Biol, 1992. 12(12): p. 5447-54.

277. Wang, G.L. and G.L. Semenza, Characterization of hypoxia-inducible factor 1 and regulation of DNA binding activity by hypoxia. J Biol Chem, 1993. 268(29): p. 21513-8.

278. Wang, G.L. and G.L. Semenza, General involvement of hypoxia-inducible factor 1 in transcriptional response to hypoxia. Proc Natl Acad Sci U S A, 1993. 90(9): p. 4304-8.

279. Carmeliet, P., et al., Role of HIF-1alpha in hypoxia mediated apoptosis, cell proliferation and tumour angiogenesis. Nature, 1998. 394: p. 485-490.

280. Barcellos-Hoff, M.H. and S.A. Ravani, Irradiated mammary gland stroma promotes the expression of tumorigenic potential by unirradiated epithelial cells. Cancer Res, 2000. 60(5): p. 1254-60.

281. Cosse, J.P. and C. Michiels, Tumour hypoxia affects the responsiveness of cancer cells to chemotherapy and promotes cancer progression. Anti-cancer agents in medicinal chemistry, 2008. 8(7): p. 790-7.

282. Dewhirst, M.W., et al., Exploring the role of HIF-1 in early angiogenesis and response to radiotherapy. Radiother Oncol, 2007. 83(3): p. 249-55.

283. Harada, H., et al., Significance of HIF-1-active cells in angiogenesis and radioresistance. Oncogene, 2007. 
284. Song, X., et al., Hypoxia-induced resistance to cisplatin and doxorubicin in nonsmall cell lung cancer is inhibited by silencing of HIF-1alpha gene. Cancer Chemother Pharmacol, 2006. 58(6): p. 776-84.

285. Liu, S. and M.S. Wicha, Targeting breast cancer stem cells. Journal of clinical oncology : official journal of the American Society of Clinical Oncology, 2010. 28(25): p. 4006-12.

286. Curiel, T.J., Immunotherapy: a useful strategy to help combat multidrug resistance. Drug Resist Updat, 2012. 15(1-2): p. 106-13.

287. Li, K., et al., Modulation of Notch signaling by antibodies specific for the extracellular negative regulatory region of NOTCH3. J Biol Chem, 2008. 283(12): p. 8046-54.

288. Fischer, M., et al., Anti-DLL4 inhibits growth and reduces tumor-initiating cell frequency in colorectal tumors with oncogenic KRAS mutations. Cancer Res, 2011. 71(5): p. 1520-5.

289. McGowan, P.M., et al., Notch1 inhibition alters the CD44hi/CD24lo population and reduces the formation of brain metastases from breast cancer. Mol Cancer Res, 2011. 9(7): p. 834-44.

290. van der Bij, G.J., et al., The perioperative period is an underutilized window of therapeutic opportunity in patients with colorectal cancer. Ann Surg, 2009. 249(5): p. 727-34.

291. Gilkes, D.M., et al., Collagen prolyl hydroxylases are essential for breast cancer metastasis. Cancer Res, 2013. 73(11): p. 3285-96.

292. Schito, L., et al., Hypoxia-inducible factor 1-dependent expression of plateletderived growth factor $B$ promotes lymphatic metastasis of hypoxic breast cancer cells. Proc Natl Acad Sci U S A, 2012. 109(40): p. E2707-16.

293. Zhang, H., et al., HIF-1-dependent expression of angiopoietin-like 4 and L1CAM mediates vascular metastasis of hypoxic breast cancer cells to the lungs. Oncogene, 2012. 31(14): p. 1757-70.

294. Elvidge, G.P., et al., Concordant regulation of gene expression by hypoxia and 2oxoglutarate-dependent dioxygenase inhibition: the role of HIF-1alpha, HIF2alpha, and other pathways. J Biol Chem, 2006. 281(22): p. 15215-26.

295. Chang, E.J., et al., Brain-type creatine kinase has a crucial role in osteoclastmediated bone resorption. Nat Med, 2008. 14(9): p. 966-72. 
296. Yan, Q., et al., The hypoxia-inducible factor 2alpha N-terminal and C-terminal transactivation domains cooperate to promote renal tumorigenesis in vivo. Molecular and cellular biology, 2007. 27(6): p. 2092-102.

297. Liu, S., et al., Breast cancer stem cells are regulated by mesenchymal stem cells through cytokine networks. Cancer Res, 2011. 71(2): p. 614-24.

298. Guenette, R.S., et al., Embigin, a developmentally expressed member of the immunoglobulin super family, is also expressed during regression of prostate and mammary gland. Dev Genet, 1997. 21(4): p. 268-78.

299. Sharrocks, A.D., et al., The ETS-domain transcription factor family. Int J Biochem Cell Biol, 1997. 29(12): p. 1371-87.

300. Oakes, S.R., et al., The Ets transcription factor Elf5 specifies mammary alveolar cell fate. Genes Dev, 2008. 22(5): p. 581-6.

301. Chakrabarti, R., et al., Elf5 inhibits the epithelial-mesenchymal transition in mammary gland development and breast cancer metastasis by transcriptionally repressing Snail2. Nat Cell Biol, 2012. 14(11): p. 1212-22.

302. Bosserhoff, A.K., et al., Functional role of melanoma inhibitory activity in regulating invasion and metastasis of malignant melanoma cells in vivo. Melanoma Res, 2001. 11(4): p. 417-21.

303. Moser, M., et al., Ultrastructural cartilage abnormalities in MIA/CD-RAPdeficient mice. Mol Cell Biol, 2002. 22(5): p. 1438-45.

304. Vasioukhin, V., et al., A novel intracellular epithelial cell tyrosine kinase is expressed in the skin and gastrointestinal tract. Oncogene, 1995. 10(2): p. 34957.

305. Born, M., et al., Simultaneous over-expression of the Her2/neu and PTK6 tyrosine kinases in archival invasive ductal breast carcinomas. J Pathol, 2005. 205(5): p. 592-6.

306. Mitchell, P.J., et al., Cloning and characterisation of cDNAs encoding a novel non-receptor tyrosine kinase, brk, expressed in human breast tumours. Oncogene, 1994. 9(8): p. 2383-90.

307. Lofgren, K.A., et al., Mammary gland specific expression of Brk/PTK6 promotes delayed involution and tumor formation associated with activation of p38 MAPK. Breast Cancer Res, 2011. 13(5): p. R89.

308. Ostrander, J.H., A.R. Daniel, and C.A. Lange, Brk/PTK6 signaling in normal and cancer cell models. Curr Opin Pharmacol, 2010. 10(6): p. 662-9. 
309. Serfas, M.S. and A.L. Tyner, Brk, Srm, Frk, and Src42A form a distinct family of intracellular Src-like tyrosine kinases. Oncol Res, 2003. 13(6-10): p. 409-19.

310. Ostrander, J.H., et al., Breast tumor kinase (protein tyrosine kinase 6) regulates heregulin-induced activation of ERK5 and p38 MAP kinases in breast cancer cells. Cancer Res, 2007. 67(9): p. 4199-209.

311. Castro, N.E. and C.A. Lange, Breast tumor kinase and extracellular signalregulated kinase 5 mediate Met receptor signaling to cell migration in breast cancer cells. Breast Cancer Res, 2010. 12(4): p. R60.

312. Locatelli, A., et al., Mechanisms of HGF/Met signaling to Brk and Sam68 in breast cancer progression. Horm Cancer, 2012. 3(1-2): p. 14-25.

313. Xiang, B., et al., Brk is coamplified with ErbB2 to promote proliferation in breast cancer. Proc Natl Acad Sci U S A, 2008. 105(34): p. 12463-8.

314. Li, X., et al., Brk/PTK6 sustains activated EGFR signaling through inhibiting EGFR degradation and transactivating EGFR. Oncogene, 2012. 31(40): p. 437283.

315. Irie, H.Y., et al., PTK6 regulates IGF-1-induced anchorage-independent survival. PLoS One, 2010. 5(7): p. el1729.

316. Wang, G.L., et al., Hypoxia-inducible factor 1 is a basic-helix-loop-helix-PAS heterodimer regulated by cellular O2 tension. Proc Natl Acad Sci U S A, 1995. 92(12): p. 5510-4.

317. Wenger, R.H. and D. Hoogewijs, Regulated oxygen sensing by protein hydroxylation in renal erythropoietin-producing cells. Am J Physiol Renal Physiol, 2010. 298(6): p. F1287-96.

318. Wenger, R.H., D.P. Stiehl, and G. Camenisch, Integration of oxygen signaling at the consensus HRE. Sci STKE, 2005. 2005(306): p. re12.

319. Bos, R., et al., Levels of hypoxia-inducible factor-1alpha independently predict prognosis in patients with lymph node negative breast carcinoma. Cancer, 2003. 97(6): p. 1573-81.

320. Yamamoto, Y., et al., Hypoxia-inducible factor lalpha is closely linked to an aggressive phenotype in breast cancer. Breast Cancer Res Treat, 2008. 110(3): $\mathrm{p}$. 465-75.

321. Prat, A. and C.M. Perou, Deconstructing the molecular portraits of breast cancer. Mol Oncol, 2011. 5(1): p. 5-23. 
322. Podo, F., et al., Triple-negative breast cancer: present challenges and new perspectives. Mol Oncol, 2010. 4(3): p. 209-29.

323. Knutson, T.P., et al., Phosphorylated and sumoylation-deficient progesterone receptors drive proliferative gene signatures during breast cancer progression. Breast Cancer Res, 2012. 14(3): p. R95.

324. Hagan, C.R., et al., ck2-dependent phosphorylation of progesterone receptors $(P R)$ on Ser81 regulates PR-B isoform-specific target gene expression in breast cancer cells. Mol Cell Biol, 2011. 31(12): p. 2439-52.

325. Graveel, C.R., et al., Met induces diverse mammary carcinomas in mice and is associated with human basal breast cancer. Proc Natl Acad Sci U S A, 2009. 106(31): p. 12909-14.

326. Kang, S.A. and S.T. Lee, PTK6 promotes degradation of c-Cbl through PTK6mediated phosphorylation. Biochem Biophys Res Commun, 2013. 431(4): p. 7349.

327. Minegishi, Y., et al., Adaptor protein complex of FRS2beta and CIN85/CD2AP provides a novel mechanism for ErbB2/HER2 protein downregulation. Cancer Sci, 2013. 104(3): p. 345-52.

328. Kamalati, T., et al., Brk, a breast tumor-derived non-receptor protein-tyrosine kinase, sensitizes mammary epithelial cells to epidermal growth factor. J Biol Chem, 1996. 271(48): p. 30956-63.

329. Pouyssegur, J., F. Dayan, and N.M. Mazure, Hypoxia signalling in cancer and approaches to enforce tumour regression. Nature, 2006. 441(7092): p. 437-43.

330. Kwon, T.G., et al., Physical and functional interactions between Runx2 and HIFlalpha induce vascular endothelial growth factor gene expression. J Cell Biochem, 2011. 112(12): p. 3582-93.

331. Schlesinger, Y., et al., Polycomb-mediated methylation on Lys27 of histone H3 pre-marks genes for de novo methylation in cancer. Nat Genet, 2007. 39(2): $\mathrm{p}$. 232-6.

332. Llor, X., et al., BRK/Sik expression in the gastrointestinal tract and in colon tumors. Clin Cancer Res, 1999. 5(7): p. 1767-77.

333. Hu, C.J., et al., Differential roles of hypoxia-inducible factor lalpha (HIF-1alpha) and HIF-2alpha in hypoxic gene regulation. Mol Cell Biol, 2003. 23(24): p. 9361-74. 
334. Raval, R.R., et al., Contrasting properties of hypoxia-inducible factor 1 (HIF-1) and HIF-2 in von Hippel-Lindau-associated renal cell carcinoma. Mol Cell Biol, 2005. 25(13): p. 5675-86.

335. Schodel, J., et al., High-resolution genome-wide mapping of HIF-binding sites by ChIP-seq. Blood, 2011. 117(23): p. e207-17.

336. Ludyga, N., et al., Effects of simultaneous knockdown of HER2 and PTK6 on malignancy and tumor progression in human breast cancer cells. Mol Cancer Res, 2013.

337. van 't Veer, L.J., et al., Gene expression profiling predicts clinical outcome of breast cancer. Nature, 2002. 415(6871): p. 530-6.

338. Khanna, C. and K. Hunter, Modeling metastasis in vivo. Carcinogenesis, 2005. 26(3): p. 513-23.

339. Xia, X. and A.L. Kung, Preferential binding of HIF-1 to transcriptionally active loci determines cell-type specific response to hypoxia. Genome Biol, 2009. 10(10): p. R113.

340. Hennipman, A., et al., Tyrosine kinase activity in breast cancer, benign breast disease, and normal breast tissue. Cancer Res, 1989. 49(3): p. 516-21.

341. Schweppe, R.E., T.H. Cheung, and N.G. Ahn, Global gene expression analysis of ERK5 and ERK1/2 signaling reveals a role for HIF-1 in ERK5-mediated responses. J Biol Chem, 2006. 281(30): p. 20993-1003.

342. Hanahan, D. and R.A. Weinberg, Hallmarks of cancer: the next generation. Cell, 2011. 144(5): p. 646-74.

343. Ai, M., et al., Brk/PTK6 cooperates with HER2 and Src in regulating breast cancer cell survival and epithelial-to-mesenchymal transition. Cancer Biol Ther, 2013. 14(3). 


\title{
APPENDIX. BREAST TUMOR KINASE (BRK/PTK6) IS A MEDIATOR OF HYPOXIA/HIF-ASSOCIATED BREAST CANCER PROGRESSION. ${ }^{\S}$
}

\begin{abstract}
Triple negative (basal type) breast cancers (TNBC) are aggressive and difficult to treat relative to luminal type breast cancers. These tumors frequently express abundant Met receptors and are enriched for transcriptional targets regulated by hypoxia inducible factor 1-alpha (HIF-1 $\alpha$ ), a principal mediator of cell stress that independently predicts cancer relapse and increased risk of metastasis. We recently showed that breast tumor kinase (Brk/PTK6) is a critical downstream effector of Met signaling and required for HGF-induced cell migration. Herein, we examined the regulation of Brk by HIFs in TNBC cell lines and mouse models. Brk mRNA and protein levels are strongly upregulated by hypoxia, low glucose and reactive oxygen species in vitro. HIF knockdown demonstrated that BRK expression is dependent upon both HIF-1 $\alpha$ and HIF$2 \alpha$, and chromatin-immunoprecipitation (ChIP) assays revealed that Brk is a direct transcriptional target of HIF- $1 \alpha / 2 \alpha$. Notably, knockdown of HIF- $1 \alpha / 2 \alpha$ in MDA-MB231 cells significantly diminished xenograft growth; Brk re-expression reversed this effect. Finally, we crossed WAP-Brk (FVB) transgenic mice into the MET $^{\text {mut }}$ knock-in (FVB) model. Expression of the Brk transgene augmented MET $^{\text {mut }}$-induced mammary tumor formation and metastasis. Surprisingly, mouse mammary tumors that arose in either MET $^{\text {mut }}$ or WAP-Brk X MET ${ }^{\text {mut }}$ mice expressed abundant Sik, the mouse homolog of Brk, which conferred markedly increased tumor formation and decreased survival. These results identify HIF- $1 \alpha / 2 \alpha$ as novel regulators of Brk expression and suggest that Brk is a key mediator of hypoxia-induced breast cancer progression. Targeting Brk expression or activity may provide an effective means to block the progression of aggressive breast cancers.
\end{abstract}

\section{Introduction}

Breast tumor kinase (Brk), also known as PTK6, is a soluble protein tyrosine kinase typically expressed in differentiated epithelial cells of the skin and gastrointestinal tract [304]. While Brk is not found in normal mammary tissue, it is aberrantly expressed in up to $86 \%$ of breast tumors, with the highest levels in advanced tumors, suggesting a tumor-specific role of this kinase in breast cancer [305-307]. Other cancers, such as melanoma, lymphoma, ovarian, prostate and colon cancer also exhibit overexpressed and/or mislocalized Brk (reviewed in [308]).

Brk contains N-terminal src homology 2 (SH2), src homology 3 (SH3) and Cterminal kinase domains. It is distantly related to Src family kinases as they share $56 \%$

$\S$ Adapted with permission. Anderson, T.M., Peacock, D.L., et al (2013). "Breast Tumor Kinase (Brk/PTK6) Is a Mediator of Hypoxia-Associated Breast Cancer Progression." Cancer Res. 2013 Aug 8. [Epub ahead of print]. 
homology within the kinase domain [309]. One notable difference between Brk and Src family kinases is that Brk lacks a myristoylation site, and is present in both the cytoplasm and the nucleus. Many Brk substrates, both cytoplasmic and nuclear, have important functions in cancer, including signal transducer and activator of transcription (STAT) molecules, Akt, and Sam68 (reviewed in [308]). Brk is activated downstream of ErbB family receptors and Met receptors and is required for EGF-, heregulin-, and hepatocyte growth factor (HGF)-enhanced cell migration [310-312]. Although Brk and ErbB2 have distinct gene loci, they are coamplified in some breast cancers [313], potentially leading to enhanced MAPK signaling and cell proliferation. Brk expression in ErbB2-induced tumors correlates with shorter latency and resistance to the ErbB2 inhibitor, Lapatinib [313]. Moreover, elevation of Brk expression in breast cancer cells confers resistance to the EGFR-blocking antibody, cetuximab, by inhibiting EGFR degradation [314]. Brk also mediates anchorage-independent growth in breast cancer cells through modulation of the IGF receptor [315]. Indeed, Brk upregulation appears to be an early event associated with growth factor-dependent tumor progression [311,313-315]. While significant advancements have been made toward understanding the mechanisms of Brk signaling [308], little is known about the regulation of Brk expression in breast cancers.

Hypoxia-inducible factors (HIFs) are the principal mediators of transcriptional responses to cellular hypoxia [316]. Hypoxia-inducible factors (HIF-1 and HIF-2) are heterodimers of two oxygen-regulated subunits, HIF- $1 \alpha$ or HIF- $2 \alpha$ and HIF-1 $\beta$. HIF-1 $\beta$ is constitutively expressed, whereas HIF $\alpha$ subunits are continually degraded through the ubiquitin pathway under normal oxygen tensions (normoxia). In contrast, in response to hypoxia, HIF $\alpha$ subunits are stabilized and translocate to the nucleus, where they can heterodimerize with HIF-1 $\beta$. HIF transcription factors recognize a core hypoxia-response element (HRE) consensus motif of 5'-RCGTG-3' within enhancer regions of target genes $[317,318]$, and act as master regulators of many cellular functions relevant to cancer progression, including angiogenesis, glucose metabolism, and tumor growth and metastasis [316]. Indeed, HIF-1 $\alpha$ is overexpressed in many human cancers (reviewed in [25]), and over-expression in breast tumors predicts relapse and indicates a higher risk of metastasis [85]. HIF-1 $\alpha$ levels are significantly higher in invasive and poorly differentiated breast cancers as compared to well-differentiated cancers [233, 319, 320]. Specifically, increased levels of HIF-1 $\alpha$ mRNA and the core hypoxic transcriptional response are associated with hormone receptor negative breast cancers [92, 320].

Clinically, breast tumors are categorized based on immunohistochemistry for estrogen receptor (ER), progesterone receptor (PR) and HER2 and have also been categorized into molecular subtypes based on gene expression profiling [321]. The least aggressive luminal subtype tumors are either $\mathrm{ER}^{+} / \mathrm{PR}^{+}$(luminal $\mathrm{A}$ ) or $\mathrm{ER}^{+} / \mathrm{PR}^{\text {low/null }}$ (luminal B), while tumors lacking ER, PR and HER2, termed triple negative breast cancers (TNBC), are typically more aggressive. TNBCs largely fall into the basal and claudin-low molecular subtypes and have a worse prognosis relative to luminal breast cancers (reviewed in [322]) in part, because these patients are not candidates for targeted therapies that block ER and HER2. TNBC patients are treated with systemic chemotherapies that include cytoskeletal- or DNA-damaging agents, which can be effective, but fail to specifically target the unknown and presumably diverse molecular 
drivers of cancer metastasis. Therefore, new molecular targets for TNBC patients are desperately needed. As Brk is aberrantly expressed in both luminal and TNBC subtypes, but is not found in the normal mammary tissue, it is an attractive candidate for specific targeting of invasive breast cancer cells.

Herein, we sought to examine the mechanism of Brk induction in breast cancer, with a specific focus on TNBC/basal-type breast cancers, which abundantly express both Met and HIF target genes. We hypothesized that Brk, a known mediator of Met signaling and stress activated kinase pathways, is directly upregulated in response to cellular hypoxia, thereby promoting cancer cell survival, cell motility, and metastasis.

\section{Materials and Methods}

\section{Generation of Modified MDA-MB-231 Cells and Cell Culture}

MDA-MB-231 cell lines were cultured in Dulbecco's modified Eagle's medium (HyClone Thermo Scientific) without pyruvate supplemented with 10\% FBS (Gibco, Invitrogen) and 1\% pen/strep. Stable knockdown of HIF $1 A, H I F 2 A$ or both genes in MDA-MB-231 cells was generated by transduction with pLKO.1-based shRNAexpressing lentiviruses as previously described for MCF-7 cells (see Supplemental Methods) [233]. MDA-MB-231 shControl, shHIF1A, and shHIF2A cells were maintained in growth medium supplemented with $4 \mu \mathrm{g} / \mathrm{mL}$ puromycin, and MDA-MB231 shHIF1A/2A cells maintained in growth medium supplemented with $8 \mu \mathrm{g} / \mathrm{mL}$ puromycin, and $2 \mathrm{mg} / \mathrm{mL}$ hygromycin. MDA-MB-231 cells were authenticated 4/11/13 by SoftGenetics LLC or DDC Medical and results compared to the ATCC STR database. Cells were maintained in $5 \% \mathrm{CO}_{2}$ at $21 \% \mathrm{O}_{2}$ (normoxia, ambient air) or at $1-2 \% \mathrm{O}_{2}$ (hypoxia).

\section{Cell Proliferation Assay}

Cell proliferation was measured via the MTT (3-[4,5-dimethylthiazol-2-yl]- 2,5diphenyltetrazolium bromide) assay as previously described [323]. MDA-MB-231 cells were plated at $2.5 \times 10^{3}$ cells per well in 24-well plates and cultured in hypoxia $\left(1 \% \mathrm{O}_{2}\right)$ for the indicated amounts of time.

\section{Protein Extraction}

Flash frozen, patient-derived xenograft tissue fragments maintained by the HCI breast tumor bank resource [132] were obtained directly from Dr. Alana L. Welm. A summary of the features of the HCI tumors used in this study is included in

Supplementary Table 1. High-salt enriched whole cell lysates (HS-WCE) were prepared from HCI tumors or MDA-MB-231 tumors as previously described [127]. Whole cell lysates from cultured cells were isolated as described in [324]. Additional human tumor 
specimens were obtained from the University of Minnesota Tissue Procurement Facility's Biological Materials Procurement Network (BioNet) and histologically subtyped and processed for protein expression as previously reported [323].

\section{Immunoblotting}

Proteins were resolved on 7.5\% SDS-PAGE or 3-8\% Tris-Acetate gels and transferred to PVDF membrane. Membranes were probed with primary antibodies: HIF$1 \alpha$ (Novus Biologicals, NB100-134 or NB-100-479), HIF-2 $\alpha$ (Novus Biologicals, NB100-122), p38 (Cell Signaling, 9212), Vinculin (Sigma, V9131), Actin (Sigma, A4700), Brk (Santa Cruz, sc-1188), Sik (Santa Cruz, sc-916) or $\beta$-Tubulin (AbCam, 6046). Secondary horseradish peroxidase-conjugated antibodies (Bio-Rad or Santa Cruz) were visualized with SuperSignal West Pico or Millipore ECL substrate. Representative images of triplicate experiments are shown in each figure. Densitometry was performed via ImageJ analysis and normalized to the loading control.

\section{qPCR}

qPCR assays were performed as previously described [324]. Briefly, cells were plated at $2.5 \times 10^{5}$ cells/well in 6 well plates and cultured at normoxia for $32 \mathrm{hrs}$, then were transferred to hypoxia $\left(1 \% \mathrm{O}_{2}\right)$ for $24 \mathrm{hrs}$ or left at normoxia. Target gene expression was normalized to the expression of internal control, TATA-binding protein (TBP).

\section{ChIP Assays}

Chromatin Immunoprecipitation (ChIP) assays were performed as previously described [324]. Briefly, MDA-MB-231 cells were plated at a density of $12 \times 10^{6}$ cells per $15 \mathrm{~cm}$ cell culture dish and cultured at normoxia for $32 \mathrm{hrs}$, then were left at normoxia or transferred to $1 \% \mathrm{O}_{2}$ for $24 \mathrm{hrs}$. Lysates were immunoprecipitated (IP) overnight (18 hrs) with $2 \mu \mathrm{g}$ of HIF-1 $\alpha$ antibody (Novus Biologicals, NB100-134), HIF- $2 \alpha$ (Novus Biologicals, NB100-122), and RNA polymerase II (Covance, 8WG16) or an equal amount of rabbit $\operatorname{IgG}$.

\section{Transgenic Mice and Generation of Tumors in NOD/scid/gamma Recipients}

MMTV-PyMT+ HIF-1 wild type (WT) and knockout (KO) mammary tumors were generated as described in [127]. WAP-Brk transgenic mice [307] and Met mutant knock-in mice were generated as described [325]. Mice were monitored daily for tumor development and euthanized when tumor volume approached $1 \mathrm{~cm}^{3}$. Cultured MDA-MB231 cells were dissociated into single cells, counted and diluted into 1:1 (vol:vol) with growth-factor reduced Matrigel-Hank's balanced salt solution (HBSS) at a density of 250,000 cells per $10 \mu 1$. Cells were kept on ice until injection into the right inguinal 
mammary fat pad of 3-6-week old female NOD/scid/ILR2 $\gamma$ (NSG) recipients using a 26gauge PT2 needle mounted on a Hamilton syringe. Recipients were palpated up to two times per week, and tumor volumes were calculated by caliper measurement as described previously [126]. At experimental endpoint, tumor wet weight was also measured.

\section{Immunostaining}

Immunohistochemistry of HIF-1 WT and KO mammary formalin-fixed paraffinembedded tumor sections was performed as previously described [307]. Briefly, tissues were incubated for 1 hour at room temperature with serum-free protein block (Dako X0909). Blocked sections were incubated overnight at $4^{\circ} \mathrm{C}$ with primary anti-Sik antibody diluted 1:1000 in Dako Antibody Diluent (S0809) and developed by DAB peroxidase staining. For immunofluorescence staining, FFPE sections (5-7 um) were antigen retrieved using $1 \mathrm{x}$ citrate buffer, stained with Sik primary antibody (sc - 916) at 1:50 dilution overnight at room temperature, followed by Alexa Flour-594 (Invitrogen) secondary and mounted with VECTASHIELD (Vector Laboratories, Inc. Burlingame, CA).

\section{Kaplan-Meier Curves}

Survival analysis was done using the van't Veer microarray dataset [236]. Normalized $B r k$ expression values were divided into four quartiles: 75 tumors with high Brk expression ( $>0.074)$ and 76 tumors with lowest $B r k(<-0.036)$ expression. The y-axis (probability) is defined as the frequency of survival. Data were analyzed using the survival package within WinSTAT ${ }^{\circledR}$ for Excel.

\section{Statistical Analysis}

Unless noted elsewhere, results are presented as means +/- SEM. Statistical significance for qPCR assays was determined using unpaired Student's t-tests. Tumor xenograft growth significance over time was determined via two-way ANOVA with Bonferroni correction. Tumor latency was analyzed using Kaplan-Meier methodology and curves compared using the Mantel-Cox Log-rank test. Brk mRNA levels were assessed using the Cancer Genome Atlas (TCGA) data.

\section{Results}

\section{Brk Is Upregulated in Response to Hypoxia and Cellular Stress}

Numerous studies have demonstrated Brk overexpression in breast and other cancers relative to a limited set of normal control tissues [308]. Data from the Cancer Genome Atlas (TCGA) were analyzed via Oncomine to compare the levels of Brk mRNA 
expression in a large number of high quality samples representing both invasive ductal and invasive lobular breast cancer versus normal breast tissues. Interestingly, independent of tumor subtype, Brk expression was significantly increased in both invasive ductal carcinoma $\left(p=1.50 \mathrm{E}^{-35}\right)$ and invasive lobular carcinoma $\left(p=3.35 \mathrm{E}^{-10}\right)$, relative to normal breast tissue samples (Figure A-1A). To investigate Brk expression levels specifically in basal/TNBCs, we collected a panel of TNBC cell lines and tumors. Human tumor samples were histologically scored and processed as previously described [323]. We observed a range of Brk expression by Western blot in all TNBC cell lines and tumor samples (Figure A-1B). Additionally, Brk expression was assayed in a subset of previously described [132] xenografted tumors maintained in the Huntsman Cancer Institute (HCI) breast tumor tissue bank. All HCI TNBC and luminal B (HER2+) subtype xenografts exhibited high levels of Brk protein expression relative to luminal B (Her2-) tumors (Figure A-1C). In addition, both HIF-1 $\alpha$ and HIF-2 $\alpha$ proteins were highly enriched in the TNBC samples relative to HER2 or steroid hormone receptor positive tumors. These data confirm that Brk, HIF- $1 \alpha$ and HIF-2 $\alpha$ are co-expressed in human breast carcinomas, particularly in TNBCs.

Multiple mechanisms of Brk upregulation exist, including gene amplification [313] and Brk-induced phosphorylation and degradation of c-Cbl [326], an E3-ubiquitin ligase responsible for downregulation of numerous oncoproteins, including ErbB family members [327]. We hypothesized that Brk expression may also be induced upon cellular stress. To address this question, we exposed TNBC cells to various stresses, including hypoxia, and examined Brk protein and mRNA expression. MDA-MB-231 breast cancer cells were subjected to mild hypoxic conditions $\left(2 \% \mathrm{O}_{2}\right)$, for increasing periods of time and harvested for Western blot analysis. HIF-1 $\alpha$ protein was upregulated following exposure of MDA-MB-231 cells to low oxygen for 4-30 hours relative to cells incubated in normoxic conditions (Figure A-2A). HIF-1 $\alpha$ protein levels peaked at approximately 6 hours of hypoxia, followed by decreased but sustained expression out to 30 hours. Interestingly, Brk levels increased at 6 hours of hypoxia compared to normoxic controls, coinciding with the peak of HIF-1 $\alpha$ protein expression. Elevated Brk protein levels were maintained out to 30 hours. The same experiment was performed in non-tumorigenic immortalized MCF10A cells, previously reported to be Brk-null [328] (Figure A-2B), as well as ER ${ }^{+} / \mathrm{PR}^{+} \mathrm{MCF} 7$ and T47D breast cancer cells (data not shown). Following 24 hours of hypoxia exposure, Brk levels were consistently increased in all cell lines. These data demonstrate that hypoxic conditions induce Brk expression in a variety of breast cell lines, and that HIF-1 $\alpha$ protein levels peak slightly before the rise in Brk protein levels.

To assess the transcriptional regulation of Brk during hypoxia, we cultured Hs578T and MDA-MB-231 cells for 24 hours in 1\% $\mathrm{O}_{2}$ and examined Brk mRNA levels by quantitative real-time PCR (qPCR). Brk transcript levels increased significantly at hypoxia compared to normoxia, in both TNBC cell lines (Figure A-2C). Levels of $V E G F$, a known HIF-1 target gene, also significantly increased in both cells lines. Interestingly, transcript levels of $E R B B 2$ and Met receptor (MET), growth factor receptors known to activate Brk signaling, were also increased significantly by hypoxia in MDA-MB-231 cells. Similar results were observed in MCF7 breast cancer cells. These 
Figure A-1. Brk is upregulated in breast cancers and in human TNBC cell lines. A. Brk mRNA levels were compared using the Cancer Genome Atlas (TCGA) dataset via Oncomine, comparing normal breast tissue to invasive ductal carcinomas (IDC), and to invasive lobular carcinomas (ILC). B. Western blot analysis of Brk protein levels in TNBC cell lines the (MDA-MB-231, MDA-MB-435, DKAT, HCC1937, and HS587T) and in triple negative human tumor specimens obtained from the University of Minnesota BioNet with antibodies to Brk and p38 (loading control). C. Human breast cancer xenograft fragments derived from the HCI resource were obtained at generation 3-5. HSWCE (10 mg/lane) were subjected to Western blot analysis for Brk, HIF-1 $\alpha$, HIF-2 $\alpha$, and ß-tubulin (loading control). 


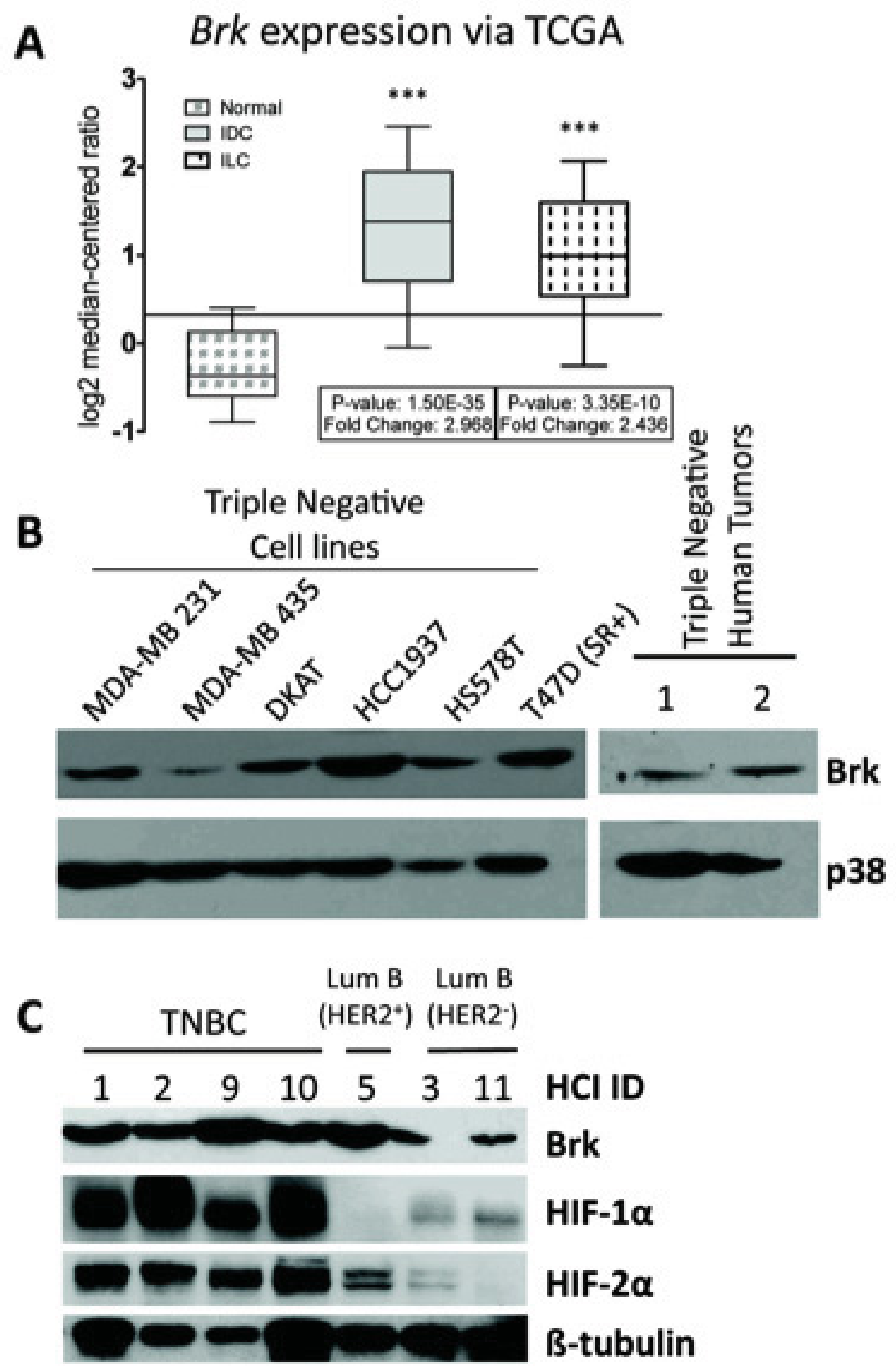




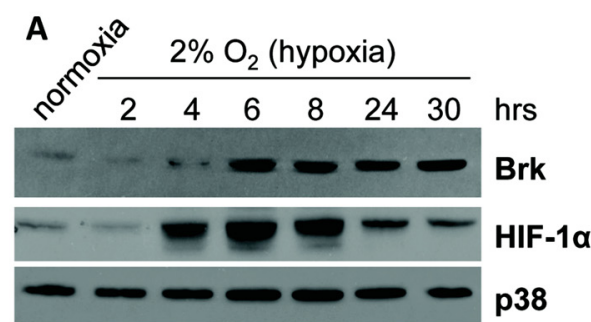

MDA-MB-231 cells
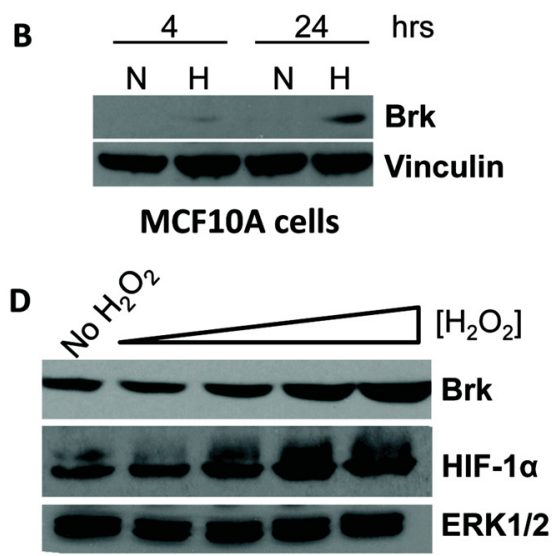

$\mathbf{E}$

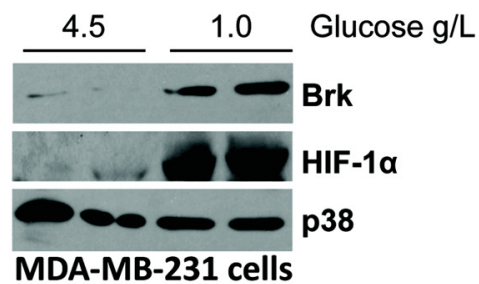

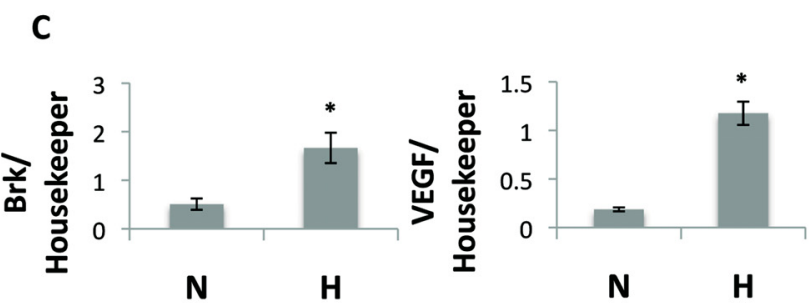

Hs578T cells
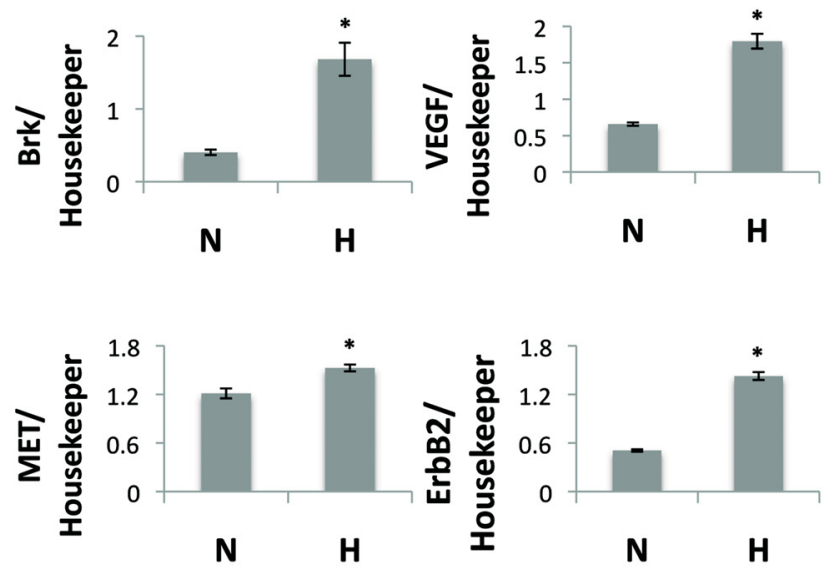

MDA-MB-231 cells

Figure A-2. Hypoxia and other cellular stresses induce Brk expression.

A. MDA-MB-231 cells were cultured in normoxia or $2 \% \mathrm{O}_{2}$ (hypoxia) for the indicated times and subjected to Western blot analysis for Brk or p38 (loading control). B.

MCF10A cells were cultured in normoxia or $2 \% \mathrm{O}_{2}$ (hypoxia) for 4 or 24 hours. Wholecell lysates were resolved on SDS-PAGE gels and probed with antibodies to Brk or vinculin (loading control). C. Hs578T and MDA-MB-231 cells were cultured at normoxia or hypoxia for 24 hours and then mRNA levels were analyzed by qPCR after normalization to TBP expression. Asterisks $(*)$ indicate statistical significance $(p<0.05$; an unpaired Student's $t$ test). D. MDA-MB-231 cells were treated with $0,1,10,50$ or 100 $\mu \mathrm{M} \mathrm{H}_{2} \mathrm{O}_{2}$. Lysates were resolved on SDS-PAGE gels and probed for antibodies specific for Brk, HIF-1 $\alpha$, and p38 (loading control). E. MDA-MB-231 cells were cultured in media with either $4.5 \mathrm{~g} / \mathrm{L}$ (DMEM-Hi) or $1.0 \mathrm{~g} / \mathrm{L}$ (DMEM-Lo) glucose. Duplicate samples (independent lysates) were resolved by SDS-PAGE and probed with antibodies to Brk, HIF-1 $\alpha$ and p38 (loading control). 
data indicate that expression of Brk, a downstream effector of the Met and ERBB2 signaling pathways, is upregulated by hypoxia. The above results suggest that Brk induction may be characteristic of more universal responses to cellular stresses that also input to HIF-1 $\alpha$ [329]. We therefore assessed the levels of Brk expression in response to increasing concentrations of reactive oxygen species (ROS) by treatment with hydrogen peroxide $\left(\mathrm{H}_{2} \mathrm{O}_{2}\right)$ and to glucose deprivation. When MDA-MB-231 cells were exposed to increasing levels of $\mathrm{H}_{2} \mathrm{O}_{2}(0-100 \mu \mathrm{M})$, Brk and $\mathrm{HIF}-1 \alpha$ protein levels were induced or stabilized, respectively, in a dose dependent manner (Figure A-2D). A similar response occurred when cells were exposed to media containing lowered glucose $(1 \mathrm{~g} / \mathrm{L})$ as compared to base DMEM-Hi media (4.5g/L) (Figure A-2E). These data indicate that cell stresses such as hypoxia, $\mathrm{H}_{2} \mathrm{O}_{2}$ exposure, and nutrient starvation are capable of stabilizing HIF- $1 \alpha$ and inducing Brk mRNA and protein over similar time courses. Brk induction by multiple cell stress pathways suggests a mechanism for coordinate regulation of downstream signaling in response to HIF activation.

\section{Brk Is a Novel, Direct HIF Transcriptional Target Gene}

We hypothesized that hypoxia/HIF signaling is directly responsible for the upregulation of Brk mRNA and protein in response to hypoxic conditions. Upon examination of the Brk promoter, we identified multiple potential hypoxia response elements (HREs) within $20 \mathrm{~kb}$ of the transcriptional start site (TSS) (Figure A-3A). To examine HIF- $\alpha$ recruitment to the Brk promoter, we performed chromatinimmunoprecipitation (ChIP) assays with MDA-MB-231 cells cultured at normoxia or hypoxia for 24 hours. We observed that HIF-1 $\alpha$ and HIF- $2 \alpha$ were robustly recruited to HRE 1 located $1.5 \mathrm{~kb}$ upstream of the Brk TSS at hypoxia compared to normoxia (Figure A-3B). As a functional correlate of transcriptional activity associated with this HRE, we assessed the recruitment of RNA polymerase II to this region (HRE 1) and observed robust recruitment of this enzyme following exposure to hypoxia (Figure A-3B). These data suggest that HIF-containing transcriptional complexes present at HRE 1 in hypoxia are active. Essentially identical results (i.e. hypoxia-regulated recruitment of HIF-1 $\alpha$, HIF-2 $\alpha$, and Pol II) were obtained for HREs 2-5. HIF-1 $\alpha$ recruitment to the VEGF promoter (Figure A-3C) was included as a positive control [330]. The first intron in the globally methylated and transcriptionally inactive hemoglobin B (HBB) gene [331] served as a negative control (Figure A-3D). These data, strongly implicate HIFs in upregulation of Brk mRNA under conditions of cellular stress in MDA-MB-231 cells.

We next sought to determine if HIF-1 $\alpha$ was required for the induction of Brk in aggressive, metastatic mammary tumors. To this end, we first examined the expression of Sik, the mouse homologue of Brk, in end-stage HIF-1 wildtype (WT) or knockout (KO) mouse mammary tumors initiated by expression of the mouse mammary virus-driven polyoma middle T oncoprotein (MMTV-PyMT) transgene, as previously described [127]. Colorimetric immunohistochemistry staining for Sik revealed a substantial loss of Sik protein expression in HIF-1 KO mammary tumors relative to WT tumors (Figure A-4A). 


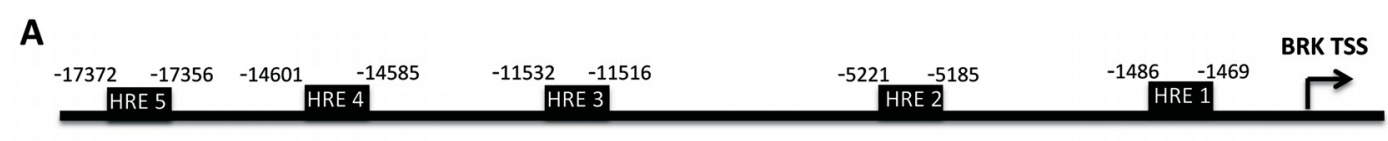

B
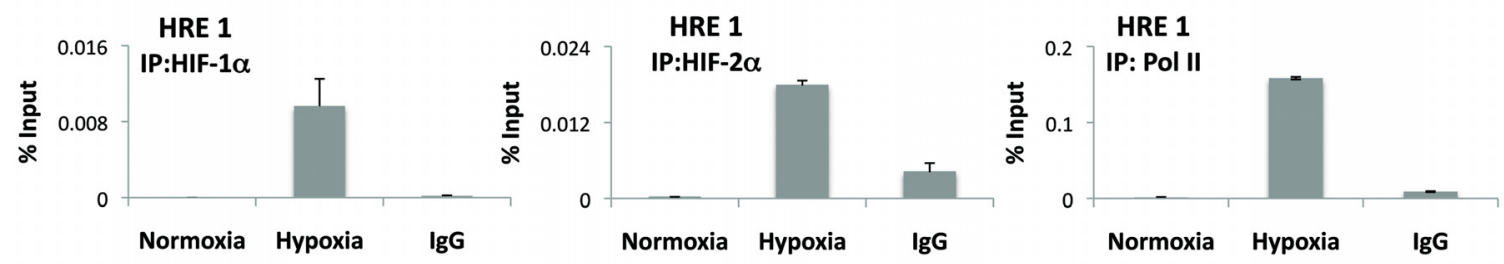

C
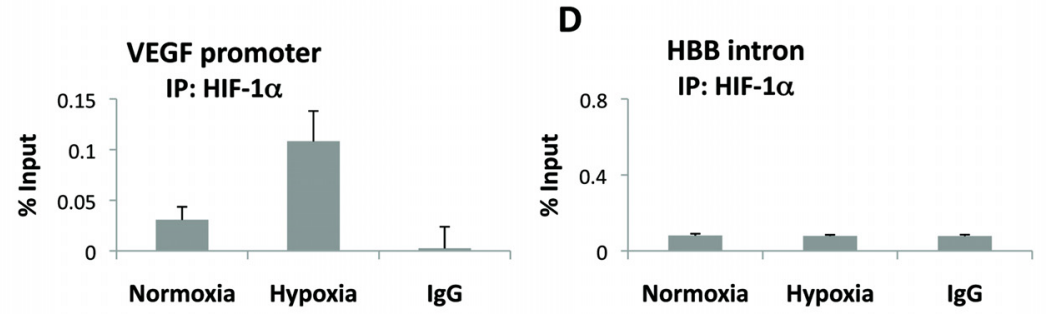

Figure A-3. Brk is a direct HIF target gene.

A. Schematic representation of HREs in the proximal Brk promoter that were assessed for HIF- $1 \alpha / 2 \alpha$ recruitment. B. MDA-MB-231 cells were cultured at normoxia or hypoxia $\left(1 \% \mathrm{O}_{2}\right)$ for 24 hours. Formaldehyde fixed lysates were subjected to ChIP with HIF-1 $\alpha$, HIF- $2 \alpha$ or RNA pol II antibodies and qPCR was performed on the isolated DNA to determine HIF and pol II recruitment to HRE 1. As controls, ChIP for HIF-1 was performed for the VEGF promoter (C), or the negative control HBB intron (D). Representative examples from triplicate experiments are shown. 


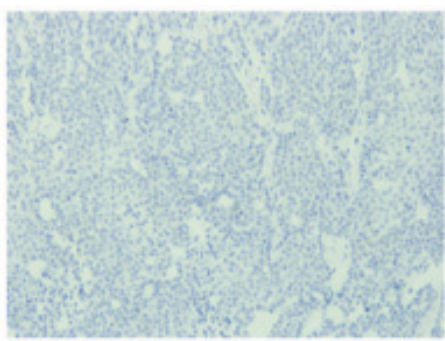

WT HIF1A tumor No $1^{\circ}$-Ab control

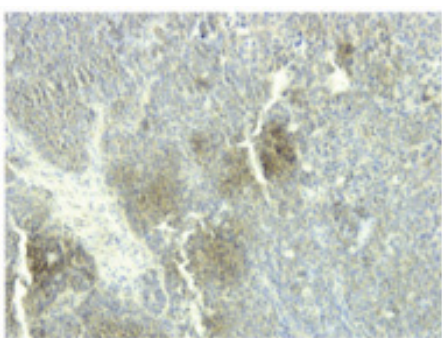

WT HIF1A tumor

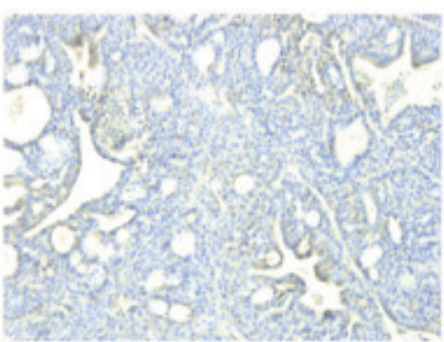

KO HIF1A tumor

B

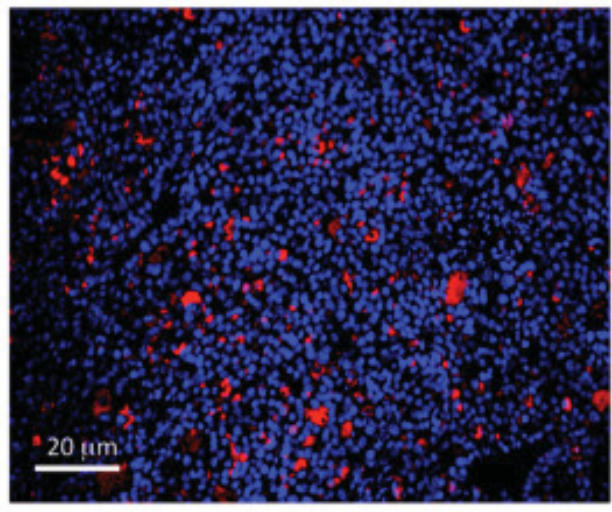

WT HIF1A tumor

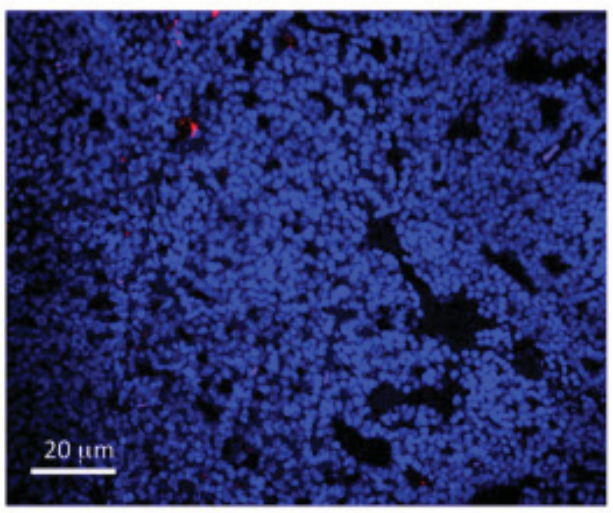

KO HIF1A tumor

C

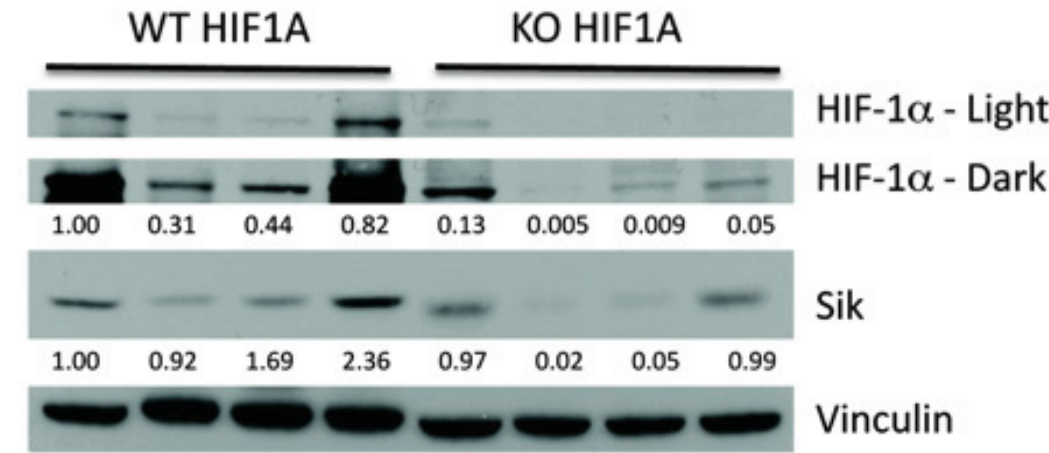

Figure A-4. Sik protein in PyMT+ murine mammary tumors is dependent upon HIF-1 $\alpha$ expression.

A. Immunohistochemistry of paraffin embedded sections from WT or HIF KO tumors stained for Sik or secondary antibody only (No 1-Ab) and developed with conventional DAB staining also revealed a dramatic decrease in Sik expression in HIF KO tumors. B. Immunofluorescence images of HIF-1 WT or KO tumors derived from the MMTVPyMT transgenic model. Paraffin embedded tissue sections were probed for Sik (detected with AlexaFluor-594, red) and stained with DAPI (blue). A no primary antibody control was run and showed no detectable staining (data not shown). C. Protein lysate from four HIF WT or HIF KO tumors were resolved by SDS-PAGE and probed with antibodies for HIF-1 $\alpha$, Sik, and vinculin as a loading control. A light and dark exposure of the HIF$1 \alpha$ blot are shown. Relative expression, via densitometry, is listed beneath. 
These results were confirmed by immunofluorescence staining for Sik (red) and DAPInuclear staining (blue) (Figure A-4B). Expression of Sik was localized in the tumor epithelium and stroma. A no primary-antibody control was included to demonstrate that all immunoreactivity was due to the primary Sik antibody (Figure A-4A); the specificity of Sik antisera was shown previously [332]. Additionally, four HIF-1 WT and four HIF-1 KO PyMT tumors were assessed for Sik protein levels by Western blotting. We observed a marked reduction in Sik protein in HIF-1 KO tumors relative to HIF-1 WT tumors (Figure A-4C). Therefore, HIF-1 $\alpha$ is required for robust expression of Sik in PyMTmouse mammary tumors. Despite dramatic reduction in HIF-1 KO PyMT-tumors, Sik protein was still weakly detected (Figure A-4). Notably, HIF-1 $\alpha$ and HIF-2 $\alpha$ have overlapping transcriptional targets [233, 333-335], although the specific role of HIF-2 $\alpha$ in breast tumors has been less well investigated, particularly in TNBCs [233]. Therefore, we tested the dependence of Brk expression on HIF $\alpha$ molecules in human breast cancer cells cultured under hypoxic conditions. MDA-MB-231 cells expressing empty vector (shControl), HIF1A shRNA, HIF2A shRNA, or both HIF1A and HIF2A shRNAs (DKD) were cultured at normoxia or $1 \% \mathrm{O}_{2}$ for 6 or 24 hours. As expected, HIF-1 $\alpha$ protein was increased at 6 and 24 hours in shControl cells cultured at hypoxia. HIF-1 $\alpha$ expression was reduced by more than $90 \%$ in hypoxic shHIF1A cells and undetectable in DKD cells (Figure A-5A). Similarly, HIF-2 $\alpha$ protein increased in hypoxia in shControl cells, but was diminished in cells expressing shRNAs targeting either HIF2A alone or both HIF factors (DKD). Notably, we observed a substantial hypoxia-induced increase in HIF-2 $\alpha$ protein expression in cells expressing shHIF1A relative to shControls. The up-regulation of HIF- $2 \alpha$ in response to efficient HIF $1 A$ knockdown was previously shown in MCF-7 cells [233]. Importantly, only double-knock down (DKD) cells simultaneously expressing both HIF1A and HIF2A shRNAs completely lacked both HIF molecules (Figure A-5A). Brk mRNA was significantly decreased in MDA-MB-231 cells expressing either shHIF1A or shHIF2A relative to shControl cells, but significantly induced by hypoxia relative to normoxia (Figure A-5B). However, when both HIF1A and HIF2A were simultaneously knocked down, we observed an almost complete ablation of Brk transcript expression. Likewise, Brk protein levels were also decreased substantially following knockdown of both HIF1A and HIF2A (Figure A-5B inset). Similar results were observed in MCF7 cells. These data suggest that when expression of either HIF-1 $\alpha$ or HIF-2 $\alpha$ is lost, the other HIF $\alpha$ subunit may compensate to induce Brk expression, consistent with the regulation of numerous other HIF-target genes, as previously shown in MCF-7 cells [233].

\section{Ectopic Brk Expression Enhances Growth of HIF1A/HIF2A Knockout Tumors in vivo}

Overexpression and enhanced activity of HIF $\alpha$ subunits is common in a variety of cancer types and has been shown to be a key mediator of tumor formation and progression $[85,95,320]$. Previous studies showed that tumors derived from MDA-MB231 cells that lack HIF-1 $\alpha$ and HIF-2 $\alpha$ displayed significantly decreased growth relative to wildtype controls $[71,292,293]$. When overexpressed in the normal mammary gland, 

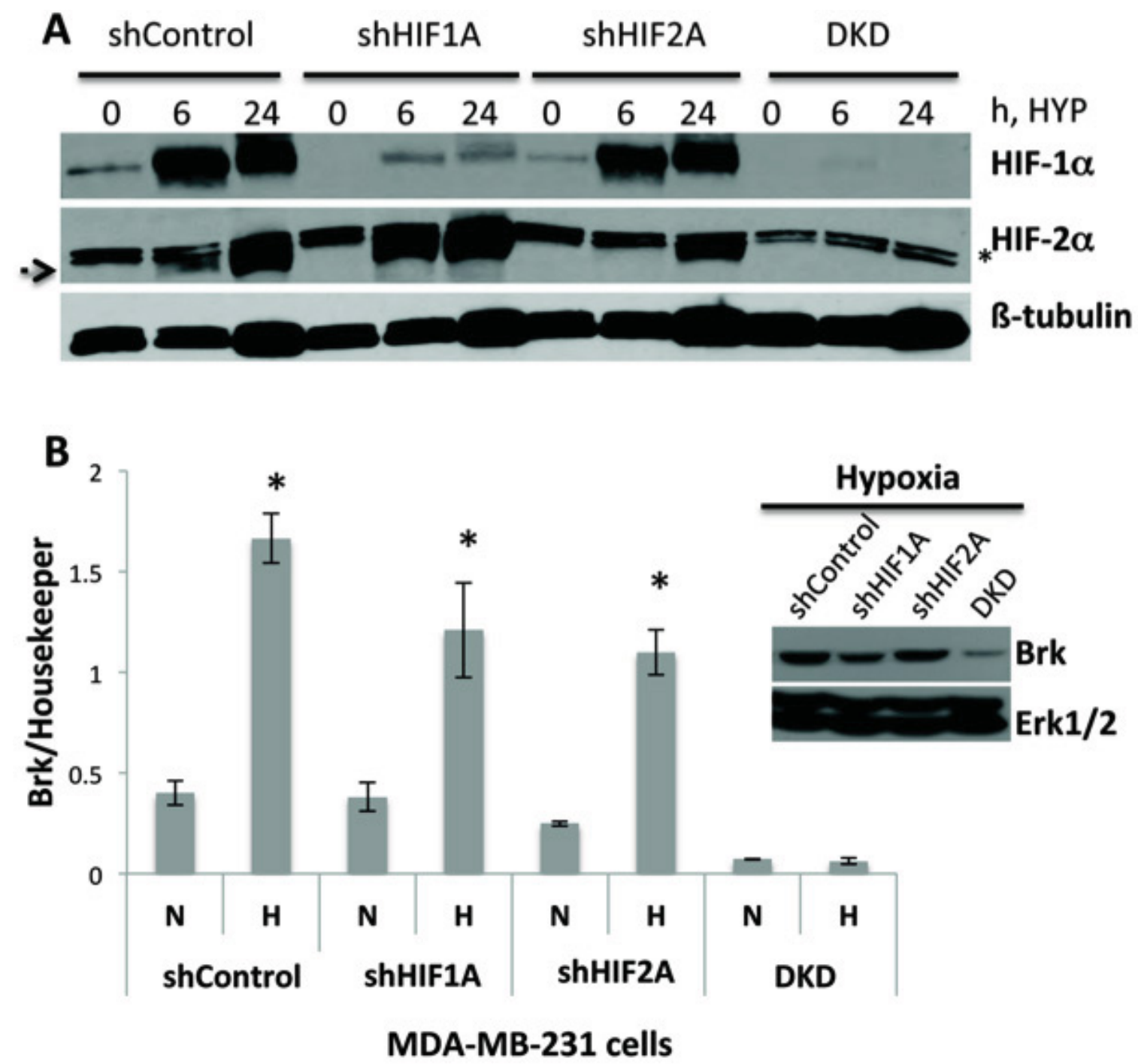

Figure A-5. Brk mRNA and protein expression are ablated upon loss of both HIF$1 \alpha$ and HIF-2 $\alpha$.

A. MDA-MB-231 cells expressing shControl, shHIF1A, shHIF2A, or DKD were cultured at normoxia or hypoxia $\left(1 \% \mathrm{O}_{2}\right)$ for 6 or 24 hours. Lysates were analyzed by Western blotting with antibodies to HIF-1 $\alpha$ or HIF- $2 \alpha$, or $\beta$-Tubulin (loading control). The asterisk indicates a cross-reactive band just above HIF-2 $\alpha$ and the arrow indicates HIF$2 \alpha$. B. MDA-MB-231 cells expressing shControl, shHIF1A, shHIF2A, or DKD were cultured at normoxia or hypoxia $\left(1 \% \mathrm{O}_{2}\right)$ for 24 hours. Protein and mRNA were isolated and mRNA was analyzed by qPCR after normalization to TBP. Inset: Western blotting analysis for Brk and ERK1/2 (loading control). 
Brk mediates delayed involution via activation of pro-survival signaling pathways and acts as a latent breast oncogene [307]. As Brk knockdown in breast cancer cells has been shown to inhibit proliferation and block cell migration in vitro [308] as well as decreasexenograft tumor size in vivo [336], we sought to determine if Brk overexpression could rescue decreased tumor growth caused by loss of HIF-1 and HIF-2 activity (i.e. a model where endogenous Brk is not HIF-induced). Thus, MDA-MB-231 cells expressing both shHIF1A and shHIF2A (DKD) cells were retrovirally infected with a pFB-neo-WTBrk overexpression construct. Western blot analysis confirmed that knockdown of $H I F 1 A$ and $H I F 2 A$ was maintained in DKD cells, and the cells were thus unable to induce endogenous Brk. In contrast, DKD cells engineered to over-express wildtype BRK exhibited constitutive Brk expression, as expected. Consistent with previously published in vivo results $[71,292,293]$, DKD cells grew significantly less than shControl cells via in vitro MTT assays. This growth defect was recovered (days 1-3) and then surpassed (day 5) upon re-expression of WT-Brk (Figure A-6A). These cells (shControl, DKD and DKD + Brk) were then used to establish orthotopic mammary tumors in NOD scid gamma female recipients $(\mathrm{n}=9 \mathrm{mice} /$ cohort $)$. Xenografts were palpated and tumor growth was measured with digital calipers as previously described [126]. In agreement with previous reports, which utilized independent shRNA targeting sequences to $H I F 1 A$ or HIF $2 A$ [71, 292, 293], knockdown of both HIF1A and HIF $2 A$ significantly decreased breast cancer cell growth in vivo relative to shControl cells at day $38 ; 780.6 \pm 98.4 \mathrm{~mm}^{3}$ vs. $558.0 \pm 36.0 \mathrm{~mm}^{3}$, respectively (Figure A-6B-C). Surprisingly, DKD cells constitutively expressing exogenous Brk (DKD + Brk) exhibited robust in vivo xenograft growth that not only reversed the HIF DKD growth phenotype $(p<0.05$ at day 35 and $p<0.001$ at day 38; day 38 mean tumor volume $921.9 \pm 88.5 \mathrm{~mm}^{3}$ vs. $558.0 \pm 36.0 \mathrm{~mm}^{3}$, respectively), but the mean tumor volume of DKD + Brk xenografts exceeded that of shControl tumors at day 38 post-transplant (Figure A-6B, $921.9 \pm 88.5 \mathrm{~mm}^{3}$ vs. $780.6 \pm$ $98.4 \mathrm{~mm}^{3}$, respectively; $p<0.05$ ). These data indicate that constitutive Brk expression is sufficient to recover the diminished growth phenotype of HIF DKD cells. In addition, comparison of mean ex vivo tumor mass confirmed that the shControl xenograft tumors were significantly larger than DKD tumors (Figure A-6C, $p=0.05$ ). Similarly, comparison of tumor wet weight revealed that DKD + Brk xenograft tumors were significantly larger than DKD tumors $(p<0.01)$. End-stage tumors were harvested and analyzed by Western blot, which confirmed the maintenance of HIF knockdown over the experimental time course (data not shown).

These results were independently repeated in a second experiment, in which we also necropsied the axillary region to detect the presence of lymph node lesions. We observed that $40 \%$ of the mice transplanted with DKD + BRK xenograft tumors harbored lymph node macrometastases $(2 / 5$ mice), with node masses greater than $2 \mathrm{~mm}$ in diameter (Figure A-6D), while no mice bearing DKD or shControl xenograft tumors exhibited overt node involvement by necropsy or H\&E analysis $(0 / 5$ mice per genotype). The presence of human breast cancer cells in these lymph nodes was confirmed by staining for human-specific anti-cytokeratin, CAM5.2, as in [132] (Figure A-6D). These data suggest that in vivo, Brk is a major driver of breast cancer phenotypes that are typically associated with HIF- $1 \alpha / 2 \alpha$ mediated tumor progression and metastasis. 

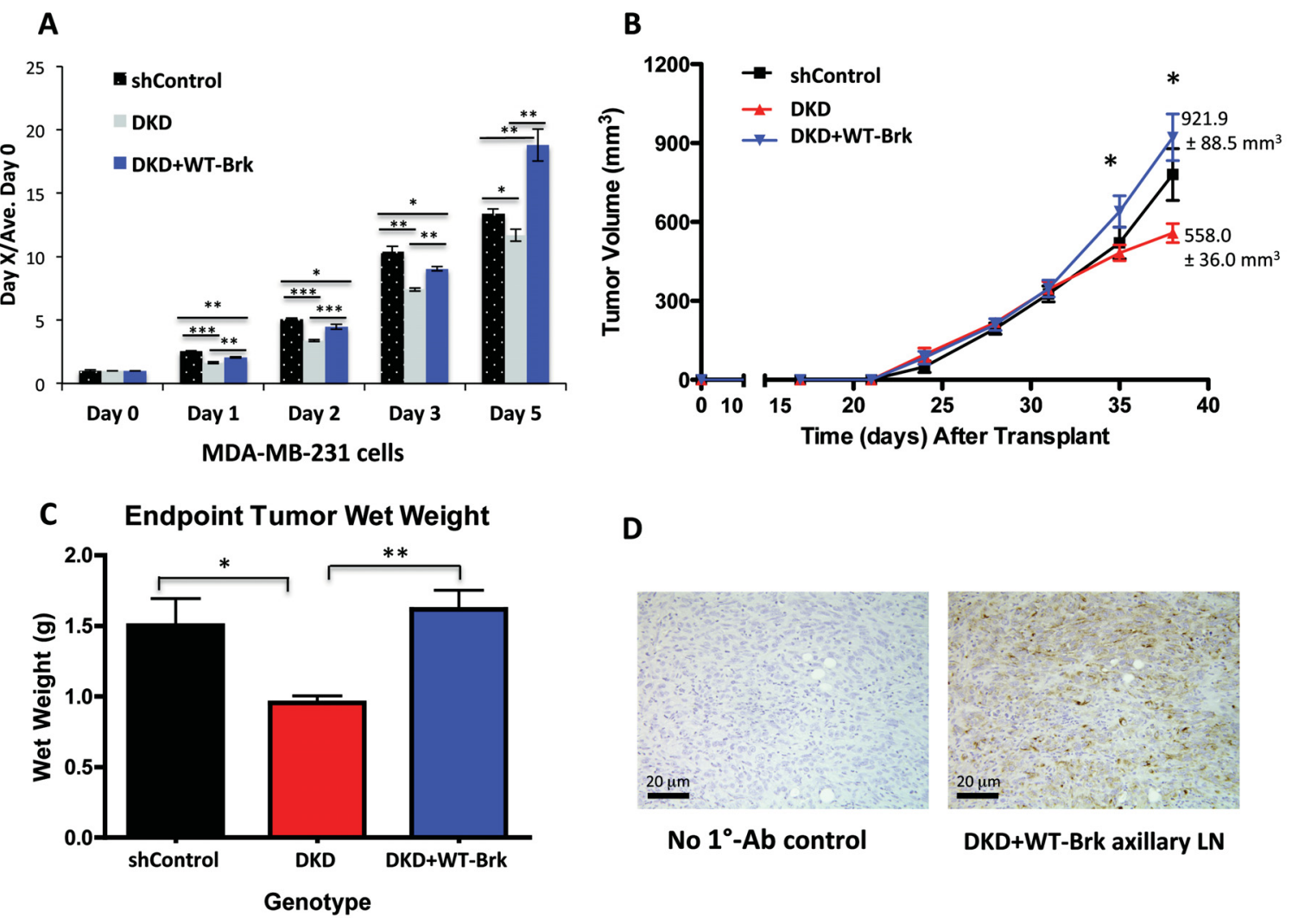

D

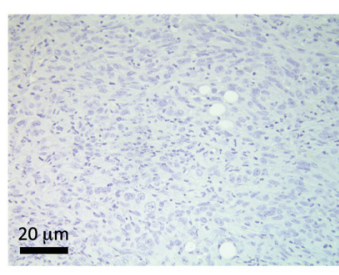

No $1^{\circ}$-Ab control

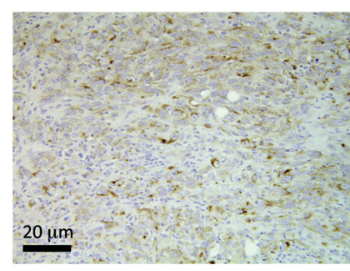

DKD+WT-Brk axillary LN

Figure A-6. Brk promotes growth in HIF DKD cells in vitro and in vivo.

A. MTT cellular proliferation assays were performed with MDA-MB-231 cells cultured at $1 \% \mathrm{O}_{2}$ for the indicated amounts of time. B. Mammary fat pad xenografts were established with either shControl, DKD or DKD + Brk MDA-MB-231 cells ( $n=9$ mice /cohort). Mean tumor volume per cohort was plotted over time; asterisks indicate significant differences via two-way ANOVA between DKD and DKD+ Brk tumors at both day 35 and day 38 post-transplant $(p<0.05, p<0.001)$. Mean tumor volumes at day 38 for DKD and DKD + Brk cohorts are listed. C. Mean ex vivo tumor wet weight. Significance was calculated via ANOVA $(* p<0.05, * * p<0.01)$. D. Representative histological images depicting CAM5.2 positive axillary lymph nodes (LN) from mice with DKD+Brk xenograft tumors. An image from a no primary antibody control is also shown (No $1^{\circ}$-Ab control). 


\section{Brk Increases the Appearance of Basal-like Mammary Tumors in MET $^{\text {mut }}$ Mice $^{-}$}

Numerous in vitro studies have implicated Brk in oncogenic signal transduction pathways that contribute to aggressive phenotypes in breast cancer cells, including migration, anchorage independent growth, and resistance to targeted therapies [311, 313315]. WAP-Brk transgenic mice exhibited increased p38 MAPK signaling associated with delayed forced involution. In addition, aged multiparous mice developed more mammary tumors relative to mice lacking mammary gland-specific Brk expression [307]. Our recent work demonstrated that Brk signals downstream of Met receptors to increase HGF-driven malignant processes in vitro [311]. Tissue microarray-based analyses of human breast cancers demonstrated that high Met receptor levels are strongly correlated with TNBC and basal-like breast cancers [325]. Therefore, we sought to determine the effects of Brk-dependent signaling on mammary tumor development in a wellcharacterized mouse model of human breast cancer. To generate mammary tumors, WAP-Brk (FVB) transgenic mice were crossed with MET $^{\text {Mut }}$ knock-in (FVB) mice, which express a constitutively active Met receptor and mimic the pathological features of human basal-like breast cancer [307, 325]. As the Met receptor is known to be an upstream activator of Brk, mammary epithelial cells derived from WAP-Brk x MET ${ }^{\mathrm{Mut}}$ mice are predicted to have constitutive activation of Brk [310, 311].

Following continuous pregnancies $(n=3)$ to activate mammary gland specific WAP-driven Brk transgene expression, groups of age-matched, multiparous female mice ( $\mathrm{n}=17$ mice per cohort; Wildtype, Brk alone, $\mathrm{MET}^{\mathrm{mut}}$ alone, or Brk x MET ${ }^{\mathrm{Mut}}$ ) were monitored for tumor formation weekly and sacrificed once tumors grew to a volume of approximately $1 \mathrm{~cm}^{3}$. Mice in the wildtype and WAP-Brk (i.e. non-activated Brk) cohorts failed to develop mammary tumors over the experimental time course (Figure A-7A). As predicted [325], nearly $100 \%$ of MET $^{\text {mut }}$ mice developed mammary tumors (by day 700). However, increased tumor formation and decreased overall tumor-free survival were observed in WAP-Brk x MET ${ }^{\mathrm{Mut}}$ mice relative to $\mathrm{MET}^{\mathrm{Mut}}$ mice $(\log$-rank $p=0.03$, Figure A-7A). The median survival of WAP-Brk x MET ${ }^{\text {Mut }}$ mice was 228 days, whereas median survival of MET $^{\text {Mut }}$ was 368 days. We collected tissues (lung, liver, brain, ovaries) from mice upon necropsy for analysis of potential (latent) metastatic events (Table A-1). Importantly, Brk x MET ${ }^{\text {Mut }}$ mice developed more (20\%) metastases relative to $\mathrm{MET}^{\mathrm{Mut}}$ mice (12\%) (Figure A-7B, Table A-1). Notably, metastases were observed in mice that survived (i.e. were not euthanized due to primary tumor size) at least 275 days; of these, 3/6 (50\%) Brk x MET ${ }^{\text {Mut }}$ mice developed distant metastases, while 2/15 (13\%) $\mathrm{MET}^{\mathrm{Mut}}$ mice developed distant metastases. Taken together, these data suggest that Brk activation in the mammary epithelium, whether driven by hypoxia or constitutive MET expression, is a driver of rapid tumor progression in vivo.

Most solid tumors are hypoxic. Our in vitro studies related to hypoxia regulation of Brk expression (Figures A2-A3) suggested that endogenous Sik expression may be induced during the course of tumor progression in $\mathrm{MET}^{\mathrm{mut}}$ mice. Therefore, all tumors extracted from mice were screened for Sik mRNA expression via qPCR. Notably, when data was stratified by Sik expression alone, regardless of Brk transgene genotype, there was a strong association between Sik positivity and decreased tumor-free survival (log- 

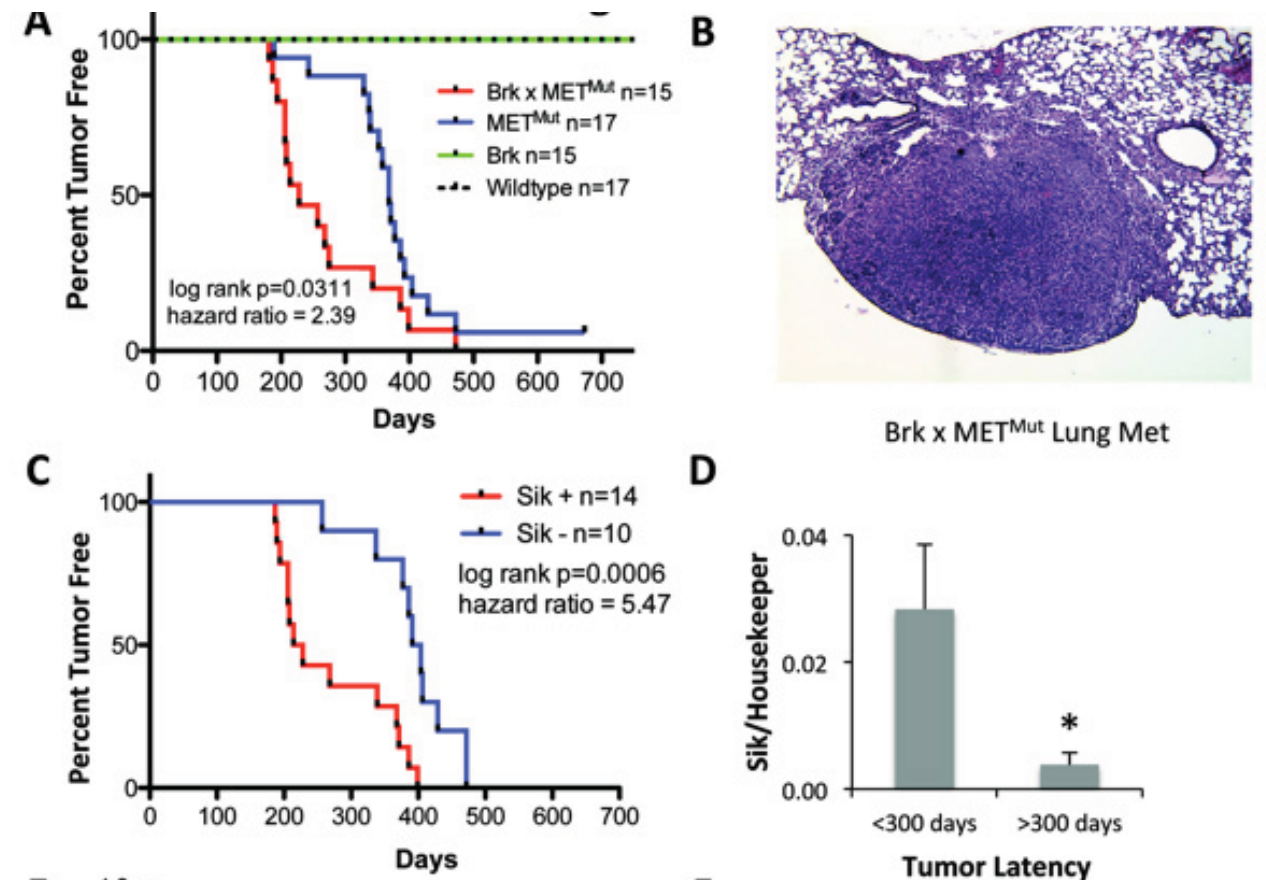

D

Brk x MET Mut Lung Met
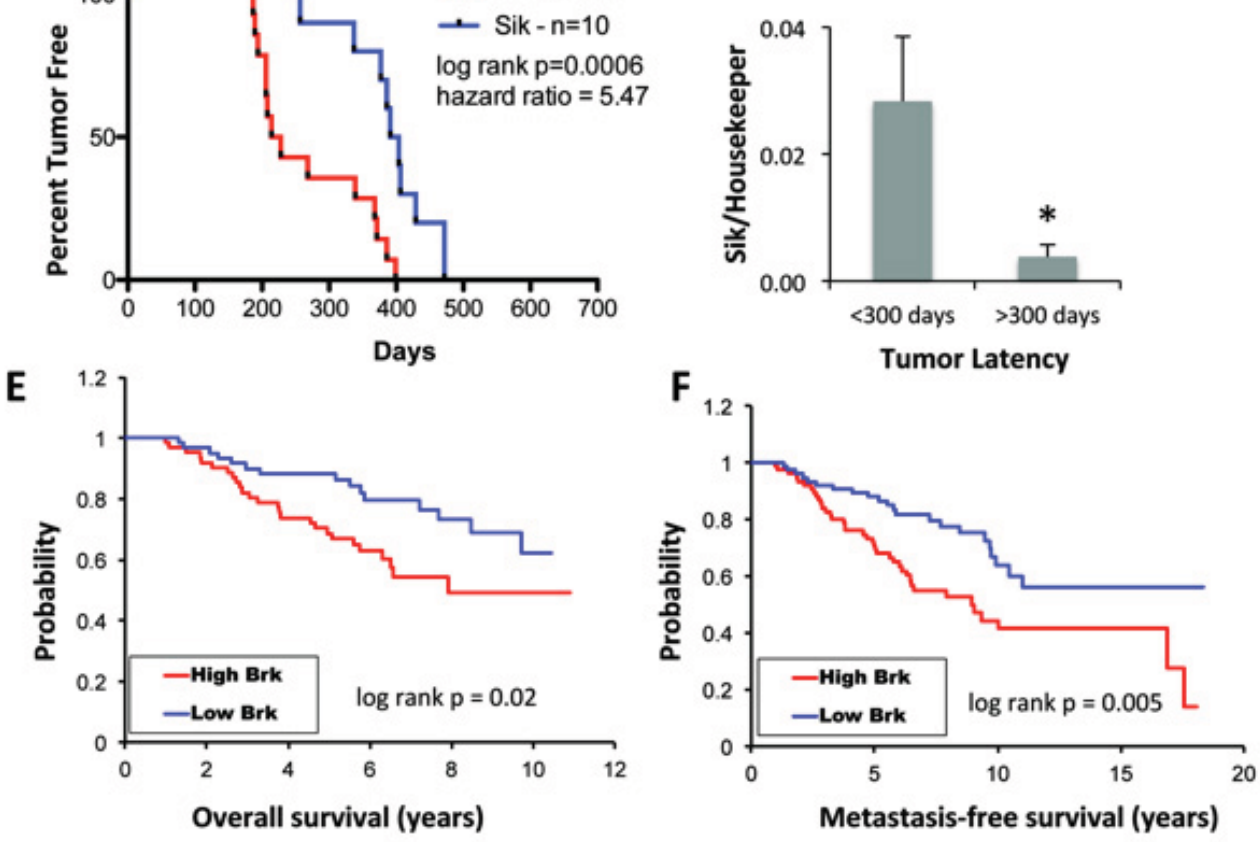

Figure A-7. Growth of basal-like mammary tumors that originate in MET $^{\text {mut }}$ mice is accelerated by expression of the Brk transgene or endogenous Sik.

A. Percent tumor-free survival of MET $^{\text {Mut }}$ mice compared to Brk x MET ${ }^{\text {Mut }}$ transgenic mice by Kaplan-Meier analysis and significance determined by Mantel-Cox Log-rank test (hazard ratio, 2.39; 95\% CI = 1.082 to 5.276). B. Representative hemotoxylin and eosin (H\&E) stained section from a Brk x MET ${ }^{\mathrm{Mut}}$ mouse lung harboring metastases is depicted. C. Percent tumor-free survival, stratified by endogenous Sik expression status. Significance was determined with Mantel-Cox Log-rank test (hazard ratio, 5.47; 95\% CI $=2.060$ to 14.50 ). D. Tumors were harvested from end-stage MET ${ }^{\text {Mut }}$ or WAP-Brk X MET $^{\text {Mut }}$ mice, and mean expression of Sik mRNA was analyzed by qPCR after normalization to TBP. Early tumors were classified as those that developed by 300 days and latent tumors were classified as those that developed after 300 days $(* p<0.05)$. E \& F. Kaplan-Meier curves were derived from data analyzed from 117 primary human breast tumor specimens [337] for overall survival (E.) and metastasis-free survival (F.), stratified by high (top quartile, $>75 \%$ ) and low (lower quartile, $<25 \%$ ) Brk expression. Significance was determined by Mantel-Cox Log-rank test. 
Table A-1. Enhanced metastasis in Brk $x$ MET $^{\text {Mut }}$ mice relative to MET $^{\text {Mut }}$ and WAP-Brk mice.

\begin{tabular}{lcccc}
\hline & \multicolumn{2}{c}{ Number of Mets/Genotype/Site } & & \begin{tabular}{c} 
Total \# of \\
\cline { 2 - 3 } Genotype
\end{tabular} \\
\cline { 2 - 3 } & Lung & Ovary & $\begin{array}{c}\text { Total \# of Mice } \\
\text { with Mets }\end{array}$ & $\begin{array}{c}\text { Mice with } \\
\text { Mets after } \\
\text { Day 275 }\end{array}$ \\
\hline WT & 0 & 0 & $0 / 15(0 \%)$ & $0 / 15(0 \%)$ \\
Brk & 0 & 0 & $0 / 17(0 \%)$ & $0 / 17(0 \%)$ \\
MET $^{\text {Mut }}$ & 2 & 0 & $2 / 17(12 \%)$ & $2 / 15(13 \%)$ \\
Brk x MET $^{\text {Mut }}$ & 3 & 1 & $3 / 15(10 \%)^{\mathrm{a}, \mathrm{b}}$ & $3 / 6(50 \%)^{\mathrm{c}}$ \\
\hline
\end{tabular}

${ }^{\mathrm{a} O n e}$ mouse had multiple organs with distant metastases.

${ }^{\mathrm{b}} p=0.26\left(\chi^{2}\right.$ test $)$.

${ }^{\mathrm{c}} p=0.02\left(\chi^{2}\right.$ test $)$.

rank $p=0.0006$ ) (Figure A-7C). Mice with Sik-positive tumors had a median survival of 221 days, whereas mice with Sik-negative tumors had a median survival of 397.5 days. Overall, the decreased latency correlated with significantly higher levels of Sik in tumors (Figure A-7C-D), suggesting that Sik expression accelerates tumor development and progression.

Collectively, our studies suggest that Brk is an important driver of breast tumor progression. Brk expression in human breast cancers may signify the need for more aggressive cancer therapies. To further support these findings as relevant to human tumors, we stratified 117 primary human breast tumor samples according to Brk mRNA expression [337]. High Brk expression correlated significantly with both decreased overall survival (Figure A-7E) and decreased metastasis-free survival (Figure A-7F). These data suggest that the WAP-Brk $x$ MET $^{\mathrm{Mut}}$ mice model human disease with high Brk expression. These mice may provide a useful model system for pre-clinical testing of novel therapies that may include Brk inhibitors.

\section{Discussion}

Although an improved understanding of Brk signaling in breast cancer is rapidly emerging [308, 310-312], the mechanisms by which Brk is aberrantly expressed in the majority of breast cancers remains undefined. We have identified cellular microenvironmental stressors, including hypoxia, to be major cues that result in increased Brk expression in both normal and neoplastic mammary epithelial cells. We have additionally shown through ChIP assays and gene expression analysis that this mechanism of Brk transcriptional regulation is HIF-dependent. This finding is particularly relevant to breast cancer subtypes that exhibit high constitutive expression of HIF $\alpha$ subunits, specifically, TNBCs (Figure A-1) and inflammatory breast cancers [92, 
320]. Importantly, forced expression of Brk in cells lacking both HIF-1 $\alpha$ and HIF-2 $\alpha$ increased xenograft growth in vivo and promoted lymph node metastasis, suggesting that Brk is a key mediator of the hypoxia-induced metastasis program. Furthermore, using a mouse model of Brk overexpression (WAP-Brk x MET ${ }^{\text {Mut }}$ knock-in), we demonstrated that activated Brk signaling drives mammary tumor initiation and rapid tumor growth in vivo. Tumor growth is further accelerated in the presence of activated endogenous Sik (the mouse homologue of Brk, which is not expressed in the normal mammary gland). Sik expression is tightly linked to rapid tumor onset and shortened survival, regardless of Brk transgene genotype. The impact of activated Brk signaling on metastatic progression is of particular interest, as $20 \%$ of the Brk x MET ${ }^{\text {Mut }}$ mice developed distant metastases, compared to only $12 \%$ of the $\mathrm{MET}^{\mathrm{Mut}}$ mice. This difference trended toward significance (Figure A-7, Table A-1), despite that our experimental design was powered for the detection of differences in primary tumor formation and growth rate rather than the detection of distant metastases (i.e. all mice are euthanized when tumors reach $1 \mathrm{~mm}^{3}$, which limits the ability to detect metastatic events derived from rapidly growing primary lesions). Clearly, high Brk expression in primary human breast tumors is significantly correlated with decreased overall survival as well as decreased metastasis-free survival (Figure A-7E-F). Further studies, designed specifically to assess differences in metastases (i.e. following removal of primary tumors and/or using larger cohorts [338]), are needed to better define the details of how Brk signaling contributes to the stepwise process of metastasis.

Our ChIP data suggest that HIF- $1 \alpha / 2 \alpha$ directly regulate Brk expression via contact with one or more distinct HREs located throughout the Brk promoter. We detected abundant recruitment of HIF- $1 \alpha / 2 \alpha$ and Pol II to at least five HREs the Brk promoter in response to hypoxia. Brk mRNA and protein were subsequently increased (Figures A-3 and A-4). In support of this finding, Sik expression was significantly diminished in mammary tumors that arose in MMTV-PyMT HIF-1 KO mice relative to WT controls. However in human MDA-MB-231 (and MCF-7) breast cancer cells, upon loss of HIF- $1 \alpha$, although the response was dampened, we still observed a net increase in hypoxia-induced Brk mRNA and protein expression. As previously shown for MCF7 cells [233], HIF-2 $\alpha$ is likely able to compensate for the loss of HIF-1 transcriptional activity in cells expressing shHIF1A, thereby maintaining Brk expression in hypoxia. Our data support the hypothesis that Brk can be up-regulated during hypoxia by either HIF-1 or HIF-2, particularly since HIF-2 $\alpha$ protein levels were indeed increased when HIF $1 \mathrm{~A}$ was knocked-down in MDA-MB-231 cells (Figure A-5A). Moreover, when both HIF-1 and HIF-2 activity is depleted using shRNAs, we observed an almost complete loss of both basal and inducible Brk mRNA and protein expression in MDA-MB-231 cells (Figure A-5) as well as MCF-7 cells. Numerous studies have determined that HIF-1 $\alpha$ and HIF-2 $\alpha$ regulate several of the same genes [233, 333, 334], as expected since the core binding sequence (RCGTG) is recognized by both HIF-1 and HIF-2 [335, 339]. Indeed, significant levels of both HIF-1 $\alpha$ and HIF- $2 \alpha$ have been observed to bind to almost all of the HIF binding sites identified by ChIP genome wide. However, functional analysis of the role of individual HREs in the regulation of Brk expression by HIFs is outside the scope of this study. Genome-wide studies have also shown that, despite sharing the same response element, each HIF $\alpha$ subunit also differentially regulates a 
unique core of distinct genes [335]. These studies highlight the complexity of HIF gene regulation and the possibility that HIF-1 $\alpha$ and HIF- $2 \alpha$ may be involved in both cooperative and compensatory gene regulation that is highly context-dependent [233], and underscore the need for further study. Additionally, molecular compensation (i.e. of one HIF for another) is an important consideration for targeting HIF-specific actions in the clinic.

Solid tumors experience widespread cell stress that acts as a form of selection pressure. Our previous studies have elucidated downstream mediators of Brk signaling that include stress-activated protein kinases (p38 MAPK and ERK5) as well as their substrates MEF2 and Sam68 [310-312]. Breast cancer cells maintain high soluble and growth factor receptor tyrosine kinase activities relative to untransformed cells [340]; these pathways may act as inputs to increased Brk and ERK5 activities [308, 314]. Intriguingly, Schweppe and co-workers have shown that HIF-1 transcriptional activity was enhanced by ERK5, and that numerous hypoxia-regulated and HIF-1 $\alpha$ target genes were also specifically regulated by ERK5 [341]. These results suggest that HGF, Met, Brk, ERK5, and HIF-1 $\alpha$ may function together as a "feed-forward" autocrine signaling loop in response to hypoxia, ultimately resulting in high levels of Brk expression and adaptation to cell stress that enables cancer cells to migrate away from hypoxic regions to more hospitable microenvironments at distant sites. Related to this process is the epithelial to mesenchymal transition (EMT), a quintessential step that precedes carcinoma cell metastasis [342]. HIF-1 is known to mediate EMT in breast tumors [127] and knock-down of Brk in MCF7 breast cancer cells partially reverses EMT, as measured by a decrease in mesenchymal markers (fibronectin and N-cadherin) and an increase in epithelial associated markers (E-cadherin and $\beta$-catenin) [343]. Taken together, these studies support the hypothesis that increased Brk expression allows breast cancer cells to successfully migrate, in part via modulation of EMT-associated molecules. Importantly, Brk appears to act as a convergence point downstream of diverse growth factor receptors that are frequently overexpressed in breast cancer. This biology, in conjunction with our findings that Brk is a major effector of in vivo HIF-mediated tumorigenesis and metastasis, suggests that targeting Brk (in addition to MET and/or HIF-dependent signaling) as part of combination breast cancer treatment strategies may more effectively subvert HIF-driven processes that contribute to aggressive tumor progression. 


\section{VITA}

Danielle Peacock was born in 1984 in Chehalis, Washington. She graduated with her Bachelor of Science from University of Washington in 2006 with a major in Biochemistry. After graduating, Danielle worked for two years in the laboratory of Dr. Kristin Swanson using mathematical models to study glioblastoma brain tumors. In 2008 she entered the Integrated Program in Biomedical Sciences (IPBS) Cancer and Developmental Biology track and the University of Tennessee Health Science Center. She then joined Dr. Tiffany Seagroves' laboratory studying the role of HIF-1 $\alpha$ in breast cancer initiation, progression and metastasis. She graduated with the degree Doctor of Philosophy in 2013 with a focus on cancer biology. 